Supporting Information

\title{
Markovnikov Hydrosilylation of Alkynes with Tertiary Silanes Catalyzed by Dinuclear Cobalt Carbonyl Complexes with NHC Ligation
}

Dongyang Wang, ${ }^{a, b, \ddagger}$ Yuhang Lai, ${ }^{a, c}, \$$ Peng Wang, ${ }^{a}$ Xuebing Leng, ${ }^{a}$ Jie Xiao,${ }^{a}$ and Liang Deng $*, a, b$

${ }^{\text {a }}$ State Key Laboratory of Organometallic Chemistry, Shanghai Institute of Organic Chemistry, Chinese Academy of Sciences, 345 Lingling Road, Shanghai 200032, China

${ }^{\mathrm{b}}$ Shenzhen Grubbs Institute, Southern University of Science and Technology, Shenzhen 518055, China

${ }^{c}$ College of Chemistry and Materials Science, Shanghai Normal University, Shanghai 200234, China *Email: deng@sioc.ac.cn 


\section{Table of Contents}

1. General Considerations and Details of Single-Crystal X-Ray Diffraction Study page S3

2. Synthesis and Characterization of Cobalt Complexes page $\mathrm{S} 4$

3. Catalyst Screening page $\mathrm{S} 13$

4. Optimization of Reaction Conditions page $\mathrm{S} 14$

5. General Procedures for the Catalytic Hydrosilylation Reactions page $\mathrm{S} 15$

6. Kinetic Profiles of the Reaction of Phenyl Acetylene with $\mathrm{HSiEt}_{3}$ Using 1-5 as Catalysts page $\mathrm{S} 30$

7. Deuterium-Labeling Experiments page $\mathrm{S} 30$

8. References page S32

9. NMR Spectra of Cobalt Complexes 2-6 and Hydrosilylation Products page S34

10. Representative GC Graphs of Hydrosilylation Reactions page $\mathrm{S} 184$ 


\section{General Considerations and Details of Single-Crystal X-Ray Diffraction Study}

General Consideration. All manipulations on air- and moisture-sensitive materials were performed under an atmosphere of dry dinitrogen with the rigid exclusion of air and moisture using standard Schlenk or cannula techniques, or in a glovebox. Solvents were dried with a solvent purification system (Innovative Technology) and degassed prior to use. NHC ligands $\operatorname{IPr}{ }^{1}{ }^{1} \operatorname{sIPr},{ }^{1}$ IMes, ${ }_{1}^{1}$ IMesMe, ${ }^{2} \mathrm{ICy},{ }^{1}\left[(\mathrm{IMes}) \mathrm{Co}_{2}(\mathrm{CO})_{7}\right]^{3}, \mathrm{PhCCD}^{4}$ and $\mathrm{DSiEt}_{3}{ }^{5}$ were synthesized according to the reported literatures. All other chemicals were purchased from chemical vendors and used as received unless otherwise noted. ${ }^{1} \mathrm{H},{ }^{2} \mathrm{H},{ }^{13} \mathrm{C}{ }^{19} \mathrm{~F}$ and ${ }^{29} \mathrm{Si}$ spectra were recorded with VARIAN $400 \mathrm{MHz}$ or Agilent $400 \mathrm{MHz}$ or BURKER $400 \mathrm{MHz}$ spectrometer at $400 \mathrm{MHz}, 61 \mathrm{MHz}, 100 \mathrm{MHz}, 376 \mathrm{MHz}$ and $79 \mathrm{MHz}$, respectively. All chemical shifts were reported in units of ppm with references to the residual protons of the deuterated solvents for proton chemical shifts, the ${ }^{13} \mathrm{C}$ of deuterated solvents for carbon chemical shifts, the ${ }^{19} \mathrm{~F}$ of $\mathrm{CFCl}_{3}$ for fluorine chemical shifts and ${ }^{29} \mathrm{Si}$ of $\mathrm{SiMe}_{4}$ for silicon chemical shifts. HRMS-EI spectra were recorded with Agilent Technologies 7250 GCQTOF instrument. HRMS-ESI spectra were recorded with Thermo Scientific Q Exactive HF Orbitrap-FTMS. HRMS-FI spectra were recorded with JEOL-AccuTOF-GCv4G-GCT MS instrument. GC analyses were performed on a Shimadzu GC-2014 spectrometer. GC-MS analyses were performed on a Shimadzu GCMS-QP2010 Plus spectrometer. Elemental analysis was performed by the Analytical Laboratory of Shanghai Institute of Organic Chemistry (CAS). IR spectra were recorded on a Bruker ALPHA II Sample Compartment RT-DLaTGS spectrophotometer. Gel permeation chromatography (GPC) analyses of the molecular weight and molecular weight distribution were performed on a Waters-GPC 50 Plus instrument using standard polystyrene as calibration and with THF as the eluent at $35^{\circ} \mathrm{C}$. Melting point was measured on a SGW X-5 apparatus and uncorrected.

Single-Crystal X-Ray Diffraction Study. Crystals of 2, 3, 4, 5.Et $2 \mathrm{O}$ and 6 were coated with Paratone- $N$ oil and mounted on a Bruker D8 Venture diffractometer equipped with an Oxford low-temperature apparatus. Cell parameters were retrieved with SMART software and refined using SAINT software on all reflections. Data integration was performed with SAINT, which corrects for Lorentz-polarization and decay. Absorption corrections were applied using SADABS. ${ }^{6}$ Space groups were assigned unambiguously by analysis of symmetry and systematic absences determined by XPREP. ${ }^{7}$ All structures were solved and refined using OLEX. The metal and first coordination sphere atoms were located from direct-methods $E$ maps. Non-hydrogen atoms were found in alternating difference Fourier synthesis and least-squares refinement cycles and during the final cycles were refined anisotropically. Hydrogen atoms were placed in calculated positions employing a riding model. CCDC 2086750-2086754 contain the supplementary crystallographic data for complexes 2, 3, 4, 5.Et $\mathbf{E} \mathbf{O}$ and $\mathbf{6}$. These data can be obtained free of charge from The Cambridge Crystallographic Data Centre via www.ccdc.cam.ac.uk/data_request/cif. 


\section{Synthesis and Characterization of Cobalt Complexes.}

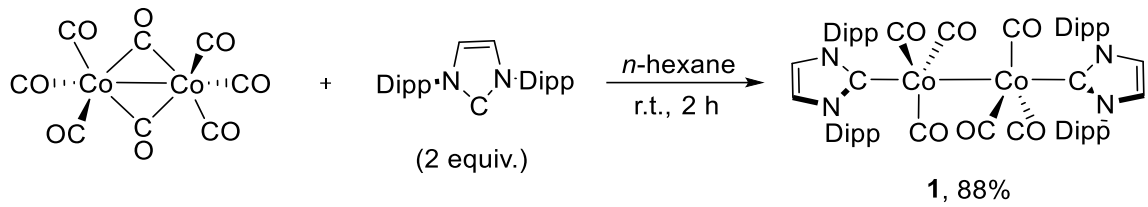

Reaction of $\mathrm{Co} 2(\mathbf{C O}) 8$ with IPr. To a solution of $\mathrm{Co}_{2}(\mathrm{CO})_{8}(680 \mathrm{mg}, 2.0 \mathrm{mmol})$ in $n$-hexane (10 $\mathrm{mL})$ was added $\operatorname{IPr}(1550 \mathrm{mg}, 4.0 \mathrm{mmol})$. After stirring for $2 \mathrm{~h}$ at room temperature, a reddish-brown suspension was formed. The mixture was filtrated. The solid was washed with $n$-hexane $(5 \mathrm{~mL})$, and then dried under vacuum, which leaves [( $\left.\operatorname{IPr})_{2} \mathrm{Co}_{2}(\mathrm{CO})_{6}\right](\mathbf{1})$ as a red brown solid $(1870 \mathrm{mg}, 88 \%)$. Single-crystals of $\mathbf{1}$ suitable for single-crystal X-ray diffraction study were obtained by slow diffusion of $n$-hexane into the THF solution of $\mathbf{1}$ at room temperature. The ${ }^{1} \mathrm{H}$ NMR spectrum of $\mathbf{1}$ prepared by this method is identical to that reported in the literature. ${ }^{8}$
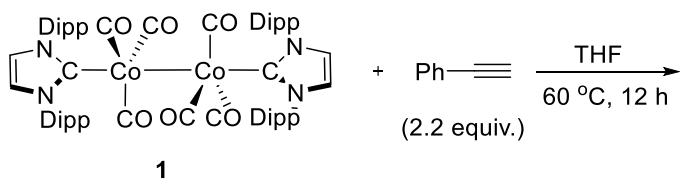

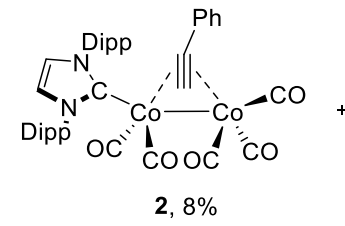

$2,8 \%$

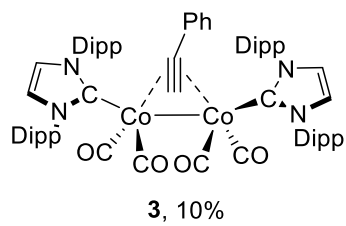

3, $10 \%$

Reaction of [(IPr) $\left.)_{2} \mathrm{Co}_{2}(\mathbf{C O})_{6}\right]$ (1) with $\mathbf{P h C} \equiv \mathbf{C H}$. To a solution of 1 (212 $\left.\mathrm{mg}, 0.20 \mathrm{mmol}\right)$ in THF $(5 \mathrm{~mL})$ was added $\mathrm{PhC} \equiv \mathrm{CH}(25 \mathrm{mg}, 0.24 \mathrm{mmol})$ at room temperature. The mixture was then heated at $60{ }^{\circ} \mathrm{C}$ and kept stirring for 12 hours. After cooling to room temperature, the mixture was subjected to vacuum to remove the volatiles. The red brown residue was extracted by $n$-hexane (20 $\mathrm{mL}$ ). The suspension was filtered to collect the brown filtrate. The filtrate was further concentrated by vacuum to $c a .2 \mathrm{~mL}$. Standing the clear solution at room temperature by slow evaporation of the solvent gives a mixture of reddish-brown crystals of $\left[(\operatorname{IPr})(\mathrm{CO})_{2} \mathrm{Co}\left(\mu-\eta^{2}: \eta^{2}-\mathrm{HCCPh}\right) \operatorname{Co}(\mathrm{CO})_{3}\right]$ (2) and purple crystals of [(IPr) $\left.(\mathrm{CO})_{2} \operatorname{Co}\left(\mu-\eta^{2}: \eta^{2}-\mathrm{HCCPh}\right) \mathrm{Co}(\mathrm{CO})_{2}(\mathrm{IPr})\right](\mathbf{3})$. The crystals of 2 and 3 can be separated by hand, and their yields are $12 \mathrm{mg}(8 \%)$ and $22 \mathrm{mg}(10 \%)$, respectively.

Characterization data of 2: ${ }^{1} \mathrm{H}$ NMR $\left(400 \mathrm{MHz}, \mathrm{C}_{6} \mathrm{D}_{6}, 298 \mathrm{~K}\right): \delta(\mathrm{ppm}) 7.50(\mathrm{~d}, 2 \mathrm{H}), 7.30(\mathrm{t}, 2 \mathrm{H})$, 7.20-7.11 (m, 4H), $7.04(\mathrm{t}, 2 \mathrm{H}), 6.96(\mathrm{t}, 1 \mathrm{H}), 6.47$ (s, 2H), 4.77 (s, 1H), 2.80 (sept, 2H), 2.52 (sept, 2H), $1.45(\mathrm{~d}, 6 \mathrm{H}), 1.11(\mathrm{~d}, 6 \mathrm{H}), 0.93(\mathrm{~d}, 6 \mathrm{H}), 0.90(\mathrm{~d}, 6 \mathrm{H}) .{ }^{13} \mathrm{C} \mathrm{NMR}\left(100 \mathrm{MHz}, \mathrm{C}_{6} \mathrm{D}_{6}, 298 \mathrm{~K}\right): \delta$ (ppm) 207.4, 202.1, 186.1, 147.9, 146.6, 140.8, 137.7, 130.9, 130.8, 126.5, 125.7, 124.5, 124.4, 113.1, 86.1, 67.9, 29.0, 28.9, 26.1, 26.0, 23.0, 22.6. Anal. Calcd for $\mathrm{C}_{40} \mathrm{H}_{42} \mathrm{Co}_{2} \mathrm{~N}_{2} \mathrm{O}_{5}$ : C, 64.17; N, 3.74; H, 5.65. Found: C, 64.19; N, 3.75; H, 5.70. IR spectrum $\left(\mathrm{KBr}, \mathrm{cm}^{-1}\right): v_{\mathrm{CO}} 2042,1999,1969$.

Characterization data of 3: ${ }^{1} \mathrm{H}$ NMR $\left(400 \mathrm{MHz}, \mathrm{C}_{6} \mathrm{D}_{6}, 298 \mathrm{~K}\right): \delta(\mathrm{ppm}) 7.40(\mathrm{~d}, 2 \mathrm{H}), 7.27(\mathrm{t}, 4 \mathrm{H})$, 7.15-7.07 (m, 11H), 6.41 (s, 4H), 3.66 (s, 1H), 2.87 (sept, 4H), 2.70 (sept, 4H), 1.30 (d, 12H), 1.17 (d, $12 \mathrm{H}), 0.97(\mathrm{~d}, 12 \mathrm{H}), 0.94(\mathrm{~d}, 12 \mathrm{H}) .{ }^{13} \mathrm{C}$ NMR (100 MHz, $\left.\mathrm{C}_{6} \mathrm{D}_{6}, 298 \mathrm{~K}\right): \delta(\mathrm{ppm}) 209.1,207.4,189.9$, 145.9, 145.8, 145.3, 137.9, 130.6, 129.7, 124.7, 124.0, 123.9, 123.5, 81.4, 62.4, 28.4, 28.3, 25.9, 25.6, 22.8, 22.6. Anal. Calcd for $\mathrm{C}_{66} \mathrm{H}_{78} \mathrm{Co}_{2} \mathrm{~N}_{4} \mathrm{O}_{4}$ : C, 71.47; N, 5.05; H, 7.09. Found: C, 71.21; N, 4.73; H, 7.32. IR spectrum $\left(\mathrm{KBr}, \mathrm{cm}^{-1}\right): v_{\mathrm{CO}} 2004,1954,1933,1902$. 


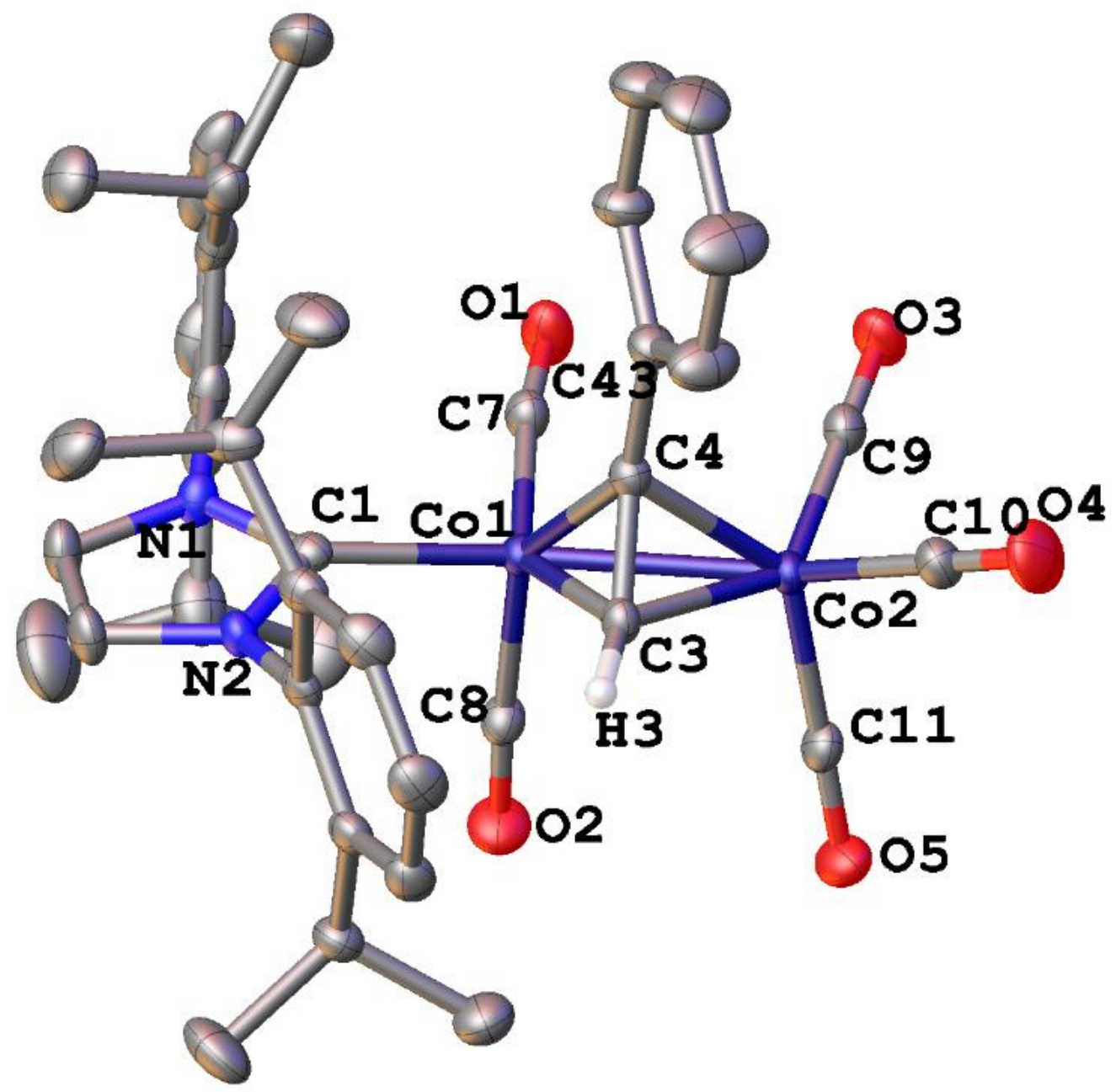

Figure S1. Molecular structure of 2 showing one of the two crystallographically independent molecules in the unit cell with $30 \%$ probability ellipsoids. Except the hydrogen atom on the bridging alkyne unit $\mathrm{CCH}$, all other hydrogen atoms were omitted for clarity. Selected bond lengths $(\AA)$ and angle ( ${ }^{\circ}$ ): Co1-Co2 2.4857(6), Co1-C1 1.961(3), Co1-C3 1.945(2), Co1-C4 1.944(2), Co2-C3 1.995(3), Co2-C4 2.017(3), C3-C4 1.329(4), Co1-C4-C43 144.6(2), Co2-C3-C43 124.9(2). 


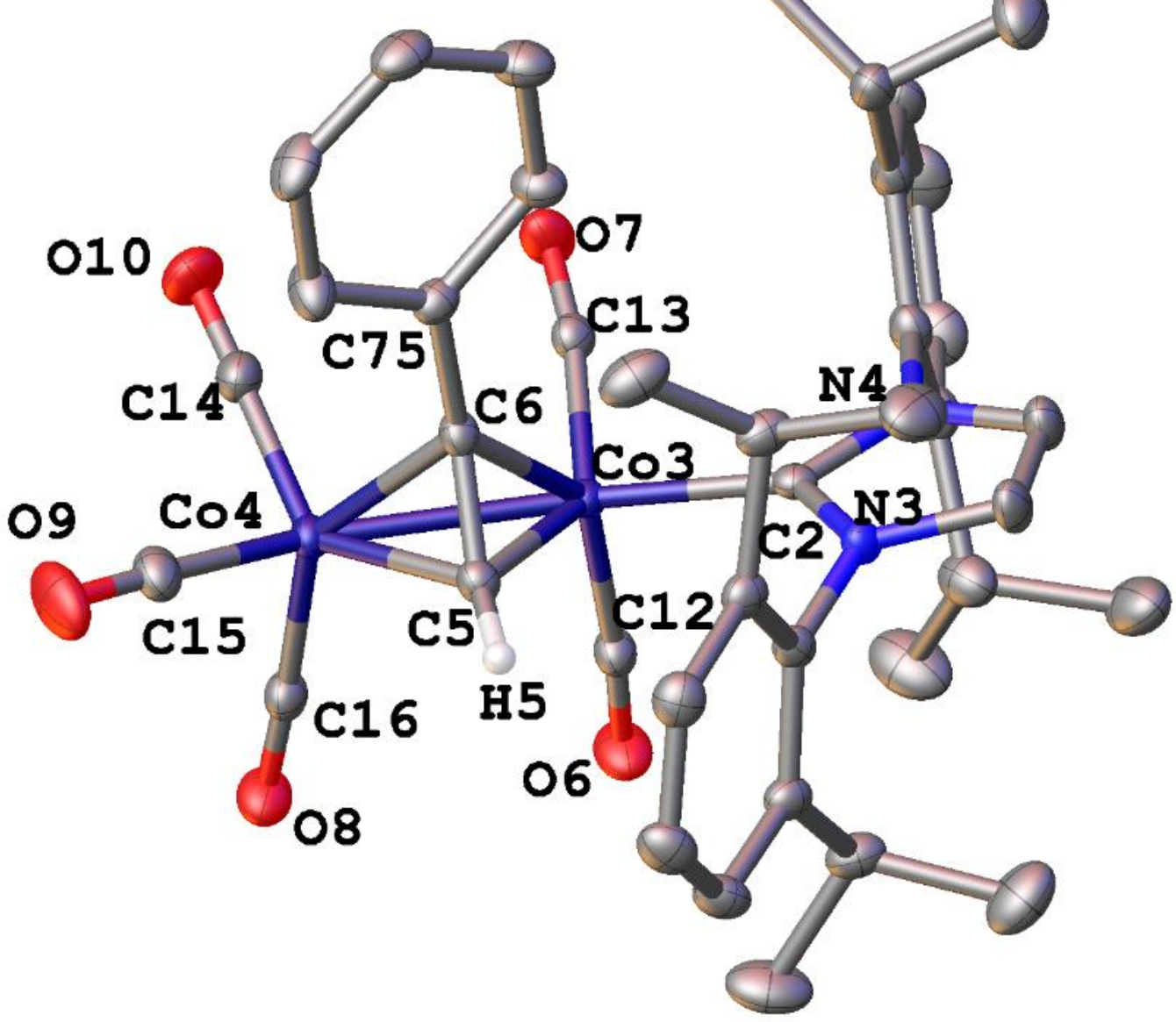

Figure S2. Molecular structure of 2 showing one of the two crystallographically independent molecules in the unit cell with $30 \%$ probability ellipsoids. Except the hydrogen atom on the bridging alkyne unit $\mathrm{CCH}$, all other hydrogen atoms were omitted for clarity. Selected bond lengths ( $\mathrm{A})$ and angle $\left({ }^{\circ}\right)$ : Co3-Co4 2.4963(6), Co3-C2 1.968(3), Co3-C5 1.936(2), Co3-C6 1.947(2), Co4-C5 1.987(3), Co4-C6 2.018(2), C5-C6 1.327(4), Co3-C6-C75 139.5(2), Co4-C6-C75 128.3(2). 


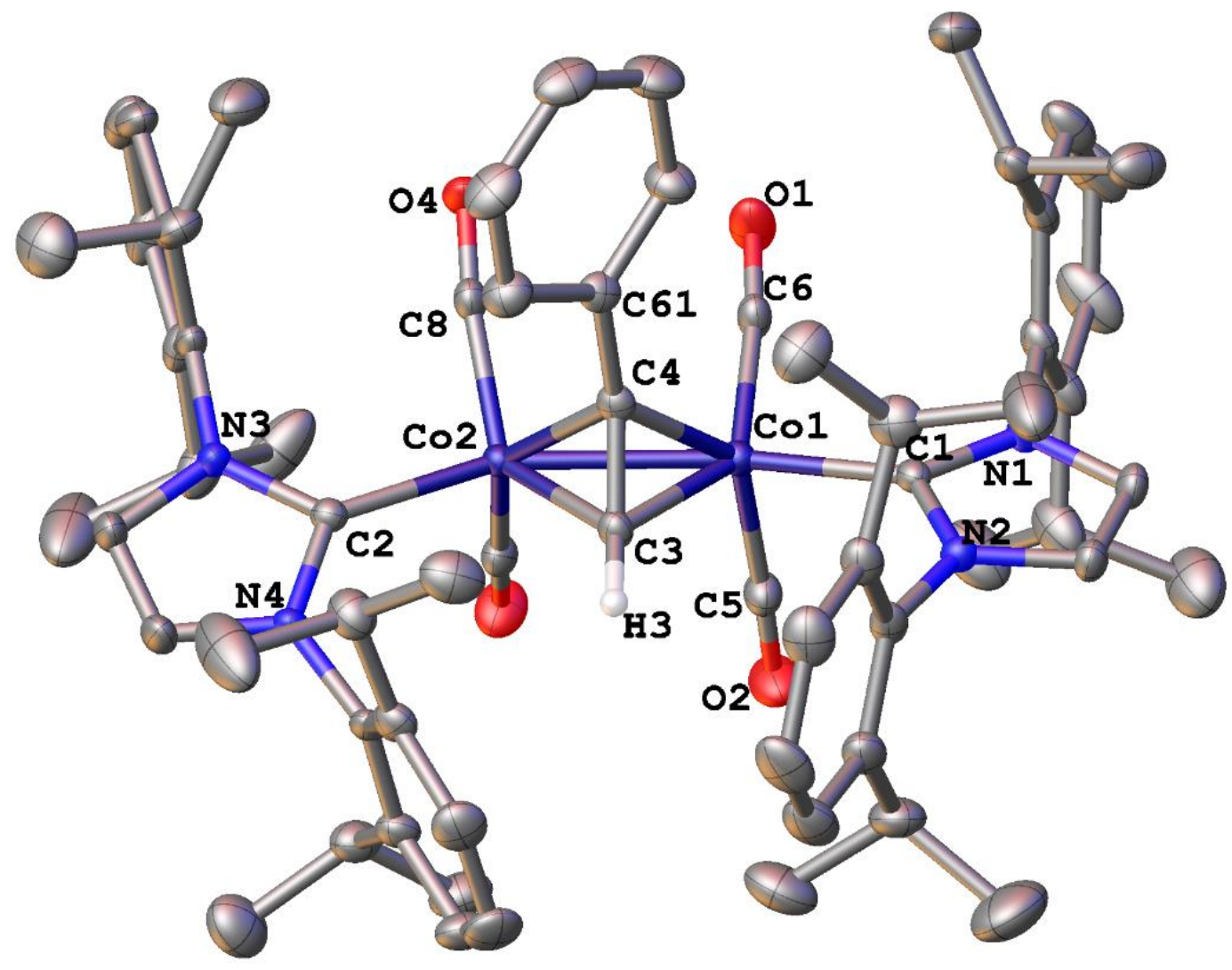

Figure S3. Molecular structure of $\mathbf{3}$ showing 30\% probability ellipsoids. Except the hydrogen atom on the bridging alkyne unit $\mathrm{CCH}$, all other hydrogen atoms were omitted for clarity. Selected bond lengths ( $⿱$ $)$ and angle $\left(^{\circ}\right)$ : Co1-Co2 2.4929(7), Co1-C1 1.986(3), Co1-C3 1.978(3), Co1-C4 1.974(3), Co2-C2 1.978(3), Co2-C4 2.005(3), Co2-C3 1.980(3), C3-C4 1.325(4), Co1-C4-C61 137.1(2), Co2-C4-C61 127.7(2).

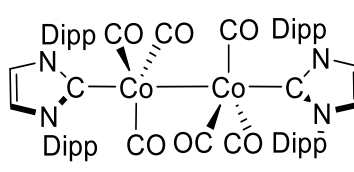

1

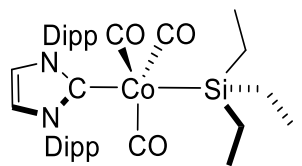

4, $50 \%$

Reaction of [(IPr) $\left.)_{2} \mathbf{C o}_{2}(\mathbf{C O})_{6}\right]$ (1) with HSiEt3. To a solution of $\mathbf{1}(212 \mathrm{mg}, 0.20 \mathrm{mmol})$ in THF $(10 \mathrm{~mL})$ was added $\mathrm{HSiEt}_{3}(47 \mathrm{mg}, 0.40 \mathrm{mmol})$ at room temperature. The mixture was then heated at $60{ }^{\circ} \mathrm{C}$ and kept stirring for 12 hours. During the course, the color of the mixture changed from reddish-brown to yellowish-brown. After cooling to room temperature, the mixture was subjected to vacuum to remove the volatiles. The residue was washed with $n$-hexane $(2 \mathrm{~mL})$ quickly and then dried under vacuum, which leaves light orange solid. The solid was then dissolved in diethyl ether (2 $\mathrm{mL})$ and filtered. Single-crystals of $\left[(\mathrm{IPr}) \mathrm{Co}(\mathrm{CO})_{3}\left(\mathrm{SiEt}_{3}\right)\right](4)$ suitable for single-crystal X-ray diffraction study was obtained by standing the clear diethyl ether solution at room temperature overnight (130 mg, 50\%). ${ }^{1} \mathrm{H}$ NMR (400 MHz, $\left.\mathrm{C}_{6} \mathrm{D}_{6}, 295 \mathrm{~K}\right): \delta(\mathrm{ppm}) 7.28(\mathrm{t}, 2 \mathrm{H}), 7.16(\mathrm{~d}, 4 \mathrm{H})$, 6.65 (s, 2H), 2.81 (sept, 4H), 1.45 (m, 12H), 1.11-1.03 (m, 27H). $\left.{ }^{13} \mathrm{C} \mathrm{NMR} \mathrm{(100} \mathrm{MHz,} \mathrm{C}_{6} \mathrm{D}_{6}, 295 \mathrm{~K}\right)$ : $\delta(\mathrm{ppm}) 202.6,192.5,146.6,137.3,130.7,125.1,124.4,28.9,25.9,22.8,11.7,9.5$. Anal. Calcd for $\mathrm{C}_{36} \mathrm{H}_{51} \mathrm{CoN}_{2} \mathrm{O}_{3} \mathrm{Si}: \mathrm{C}, 66.85 ; \mathrm{N}, 4.33 ; \mathrm{H}, 7.95$. Found: C, 66.68; N, 4.44; H, 7.85. IR spectrum (KBr, 
$\left.\mathrm{cm}^{-1}\right): v_{\mathrm{CO}} 2009,1940,1910$.

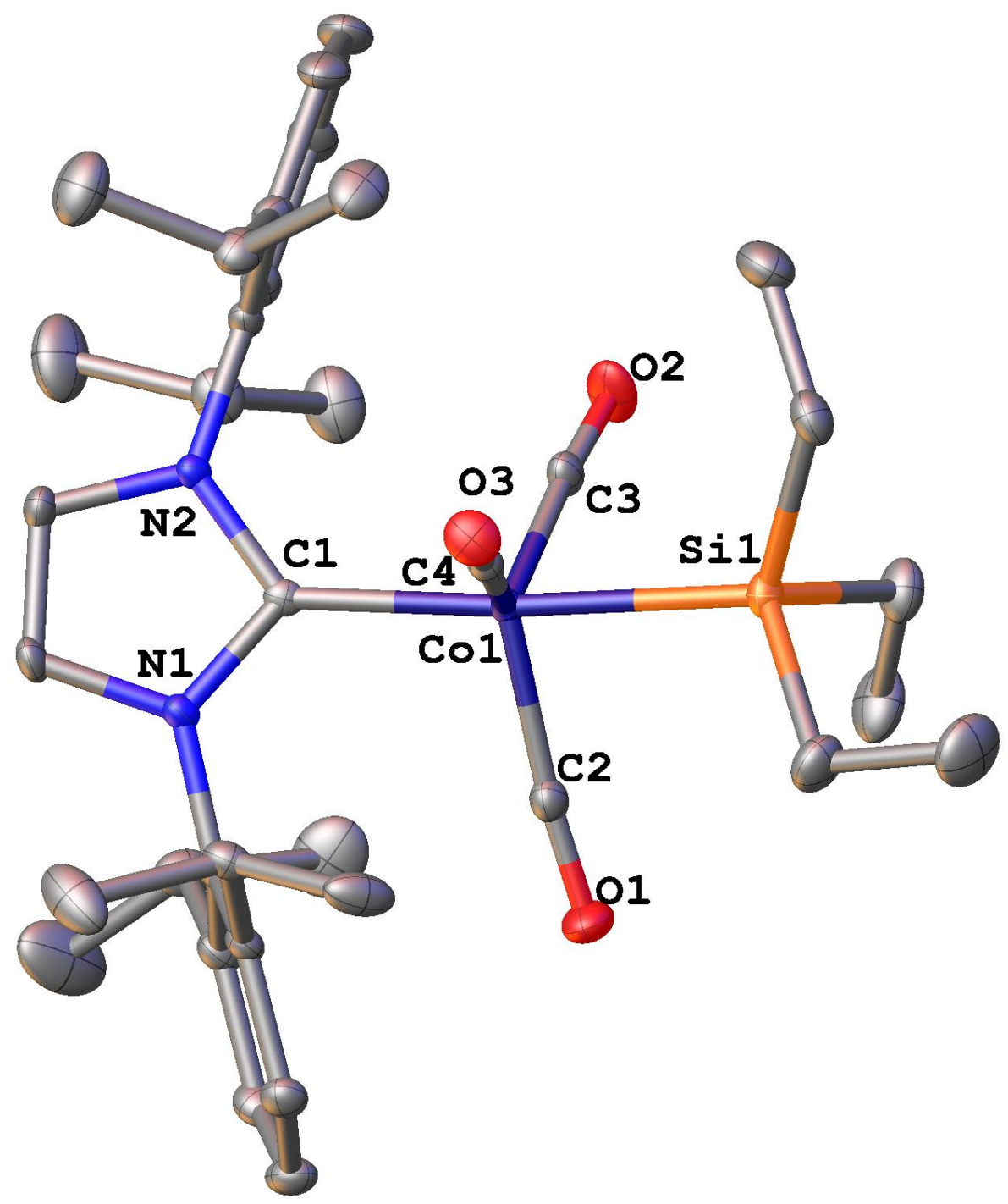

Figure S4. Molecular structure of 4 showing $30 \%$ probability ellipsoids. all hydrogen atoms were omitted for clarity. Selected bond lengths $(\AA)$ and angle $\left(^{\circ}\right)$ : Co1-C1 1.978(3), Co1-Si1 2.3587(9), C1-Co1-Si1 171.69(8).

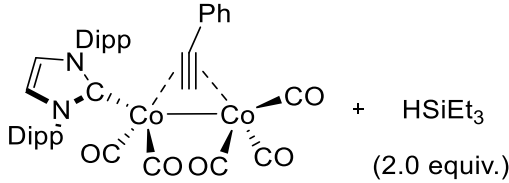

2

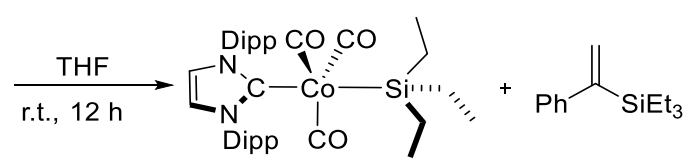

$4,82 \%$ $93 \%$

Reaction of $\left[(\mathrm{IPr})(\mathrm{CO})_{2} \mathrm{Co}\left(\mu-\eta^{2}: \boldsymbol{\eta}^{2}-\mathrm{HCCPh}\right) \mathrm{Co}(\mathrm{CO})_{3}\right]$ (2) with HSiEt 3 . To a solution of 2 (144 mg, $0.20 \mathrm{mmol})$ in THF (10 mL) was added $\mathrm{HSiEt}_{3}(46 \mathrm{mg}, 0.40 \mathrm{mmol})$. After stirring at room temperature for 12 hours, the color of the mixture changed from reddish-brown to yellowish-brown. The reaction mixture was then subjected to vacuum to remove the volatiles. The residue was washed by $n$-hexane $(2 \mathrm{~mL})$ quickly and filtered. The light orange solid was dried and characterized by NMR that indicate its identity as $\left[(\operatorname{IPr}) \mathrm{Co}(\mathrm{CO})_{3}\left(\mathrm{SiEt}_{3}\right)\right]$ (4) (106 $\left.\mathrm{mg}, 82 \%\right)$. On the other hand, 
1,3,5-trimethoxybenzene $(33 \mathrm{mg}, 0.20 \mathrm{mmol}$ ) was added to the filtrate as an internal standard for NMR yield quantification. The mixture was further subjected to vacuum to remove all the solvent. The oily residue was characterized by ${ }^{1} \mathrm{H}$ NMR, which indicated the formation of $\alpha$-triethylsilyl styrene in $93 \%$ NMR yield.

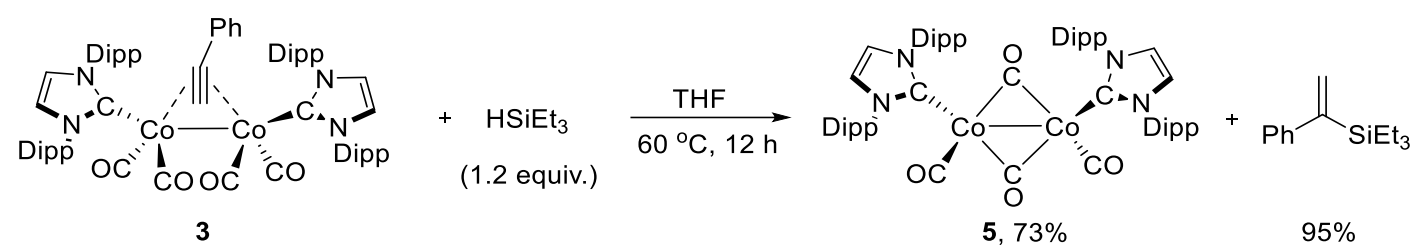

Reaction of $\left[(\operatorname{IPr})(\mathrm{CO})_{2} \mathrm{Co}\left(\mu-\eta^{2}: \eta^{2}-\mathrm{HCCPh}\right) \mathrm{Co}(\mathrm{CO})_{2}(\operatorname{IPr})\right](3)$ with $\mathrm{HSiEt}_{3}$. To a solution of $3(222 \mathrm{mg}, 0.20 \mathrm{mmol})$ in THF (10 mL) was added $\mathrm{HSiEt}_{3}(28 \mathrm{mg}, 0.24 \mathrm{mmol})$ at room temperature. The mixture was then heated at $60{ }^{\circ} \mathrm{C}$ and kept stirring for 12 hours. During the course, the color of the mixture changed from purple to yellowish-green. After cooling to room temperature, the mixture was subjected to vacuum to remove the volatiles. The residue was washed with $n$-hexane $(2 \mathrm{~mL})$ quickly and then dried under vacuum, which leaves $\left[(\mathrm{IPr}) \mathrm{Co}(\mathrm{CO})_{2}\right]_{2}(\mathbf{5})$ as a kelly solid (146 $\mathrm{mg}$, 73\%). Single-crystals of 5 suitable for single-crystal X-ray diffraction study was obtained by standing its diethyl ether solution at $-30{ }^{\circ} \mathrm{C}$ for days. On the other hand, 1,3,5-trimethoxybenzene (33 $\mathrm{mg}, 0.20 \mathrm{mmol}$ ) was added to the filtrate as an internal standard for NMR yield quantification. The mixture was further subjected to vacuum to remove all the solvent. The oily residue was characterized by ${ }^{1} \mathrm{H} \mathrm{NMR}$, which indicated the formation of $\alpha$-triethylsilyl styrene in $95 \%$ NMR yield.

Characterization data of 5: ${ }^{1} \mathrm{H}$ NMR $\left(400 \mathrm{MHz}, \mathrm{C}_{6} \mathrm{D}_{6}, 303 \mathrm{~K}\right)$ for 5: $\delta(\mathrm{ppm}) 7.08(\mathrm{t}, 4 \mathrm{H}), 6.98(\mathrm{~d}$, $8 \mathrm{H}), 6.40$ (s, 4H), 2.71 (sept, 8H), 1.30 (br, 24H), 1.17 (br, 24H). ${ }^{13} \mathrm{C}$ NMR (100 MHz, $\mathrm{C}_{6} \mathrm{D}_{6}, 303 \mathrm{~K}$ ): $\delta$ (ppm) 144.8, 138.5, 135.5, 129.7, 123.6, 28.4, 25.8, 23.9. Anal. Calcd for $\mathrm{C}_{58} \mathrm{H}_{72} \mathrm{Co}_{2} \mathrm{~N}_{4} \mathrm{O}_{4}: \mathrm{C}, 69.17$; N, 5.56; H, 7.21. Found: C, 68.87; N, 5.50; H, 7.13. IR spectrum $\left(\mathrm{KBr}, \mathrm{cm}^{-1}\right): v_{\mathrm{CO}} 1935,1899,1852$, 1799. 


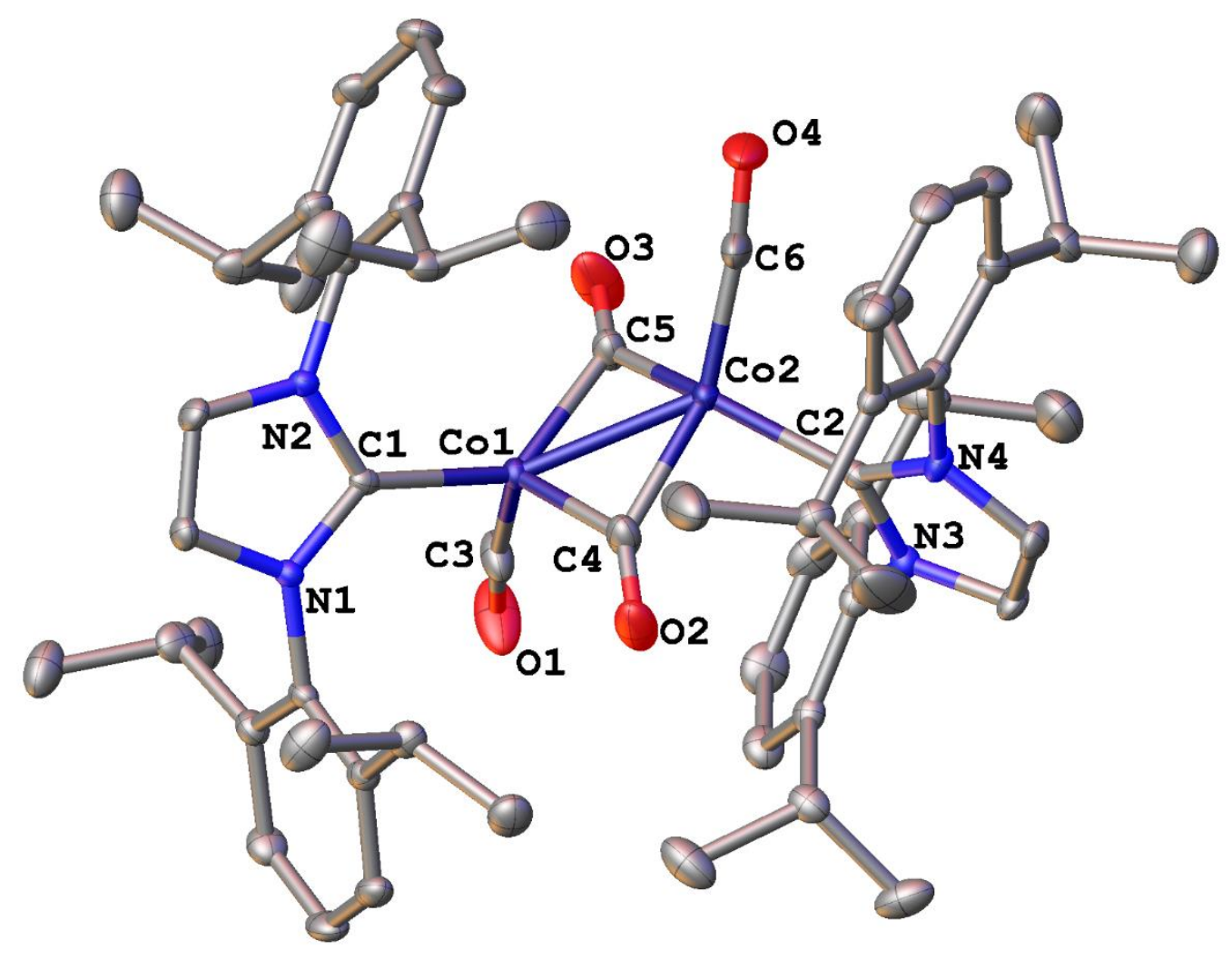

Figure S5. Molecular structure of 5 showing 30\% probability ellipsoids. all hydrogen atoms were omitted for clarity. Selected bond lengths $(\AA)$ and angle $\left({ }^{\circ}\right)$ : Co1-Co2 2.3955(6), Co1-C1 1.944(3), Co2-C2 1.935(3), C1-Co1-Co2 131.71(9), C2-Co2-Co1 130.41(9).

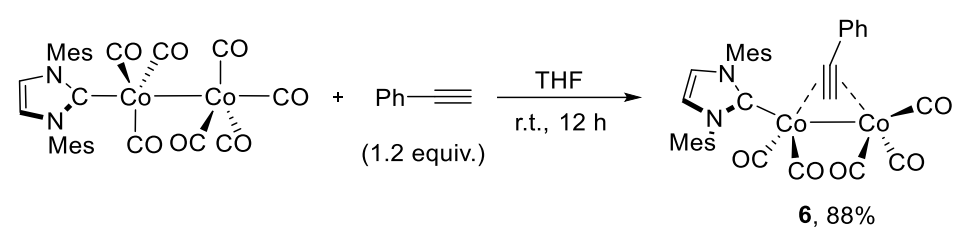

Reaction of [(IMes) $\left.\mathrm{Co}_{2}(\mathrm{CO})_{7}\right]$ with $\mathbf{P h C} \equiv \mathbf{C H}$. To a solution of [(IMes) $\left.\mathrm{Co}_{2}(\mathrm{CO})_{7}\right](124 \mathrm{mg}$, $0.20 \mathrm{mmol})$ in $\mathrm{THF}(10 \mathrm{~mL})$ was added $\mathrm{PhC} \equiv \mathrm{CH}(25 \mathrm{mg}, 0.24 \mathrm{mmol})$ at room temperature. The mixture was kept stirring at room temperature for 12 hours, giving a red brown solution. The solution was subjected to vacuum to remove the volatiles. The residue was further washed with $n$-hexane (2 $\mathrm{mL})$ and dried under vacuum, which leaves [(IMes)(CO) $\left.{ }_{2} \mathrm{Co}\left(\mu-\eta^{2}: \eta^{2}-\mathrm{HCCPh}\right) \mathrm{Co}(\mathrm{CO})_{3}\right](6)$ as a reddish brown solid (117 $\mathrm{mg}, 88 \%$ ). Single-crystals of 6 suitable for single-crystal X-ray diffraction study was obtained by standing its diethyl ether solution at $-30{ }^{\circ} \mathrm{C}$ for days. ${ }^{1} \mathrm{H}$ NMR $(400 \mathrm{MHz}$, $\left.\mathrm{C}_{6} \mathrm{D}_{6}, 303 \mathrm{~K}\right): \delta(\mathrm{ppm}) 7.62(\mathrm{~d}, 2 \mathrm{H}), 7.11(\mathrm{t}, 2 \mathrm{H}), 7.01(\mathrm{t}, 1 \mathrm{H}), 6.81(\mathrm{~s}, 4 \mathrm{H}), 5.96(\mathrm{~s}, 2 \mathrm{H}), 4.84(\mathrm{~s}, 1 \mathrm{H})$, $2.17(\mathrm{~s}, 6 \mathrm{H}), 1.94(\mathrm{~s}, 6 \mathrm{H}) .1 .73(\mathrm{~s}, 6 \mathrm{H}) .{ }^{13} \mathrm{C} \mathrm{NMR}\left(100 \mathrm{MHz}, \mathrm{C}_{6} \mathrm{D}_{6}, 303 \mathrm{~K}\right): \delta$ (ppm) 202.4, 182.4, 141.3, 139.5, 137.6, 136.2, 136.0, 130.9, 129.6, 129.5, 126.6, 123.9, 83.7, 68.5, 21.2, 18.0, 17.9. Anal. Calcd for $\mathrm{C}_{34} \mathrm{H}_{30} \mathrm{Co}_{2} \mathrm{~N}_{2} \mathrm{O}_{5}$ : C, 61.46; N, 4.22; H, 4.55. Found: C, 61.29; N, 4.21; H, 4.51. IR spectrum $\left(\mathrm{KBr}, \mathrm{cm}^{-1}\right): v_{\mathrm{CO}} 2050,1994,1976$. 


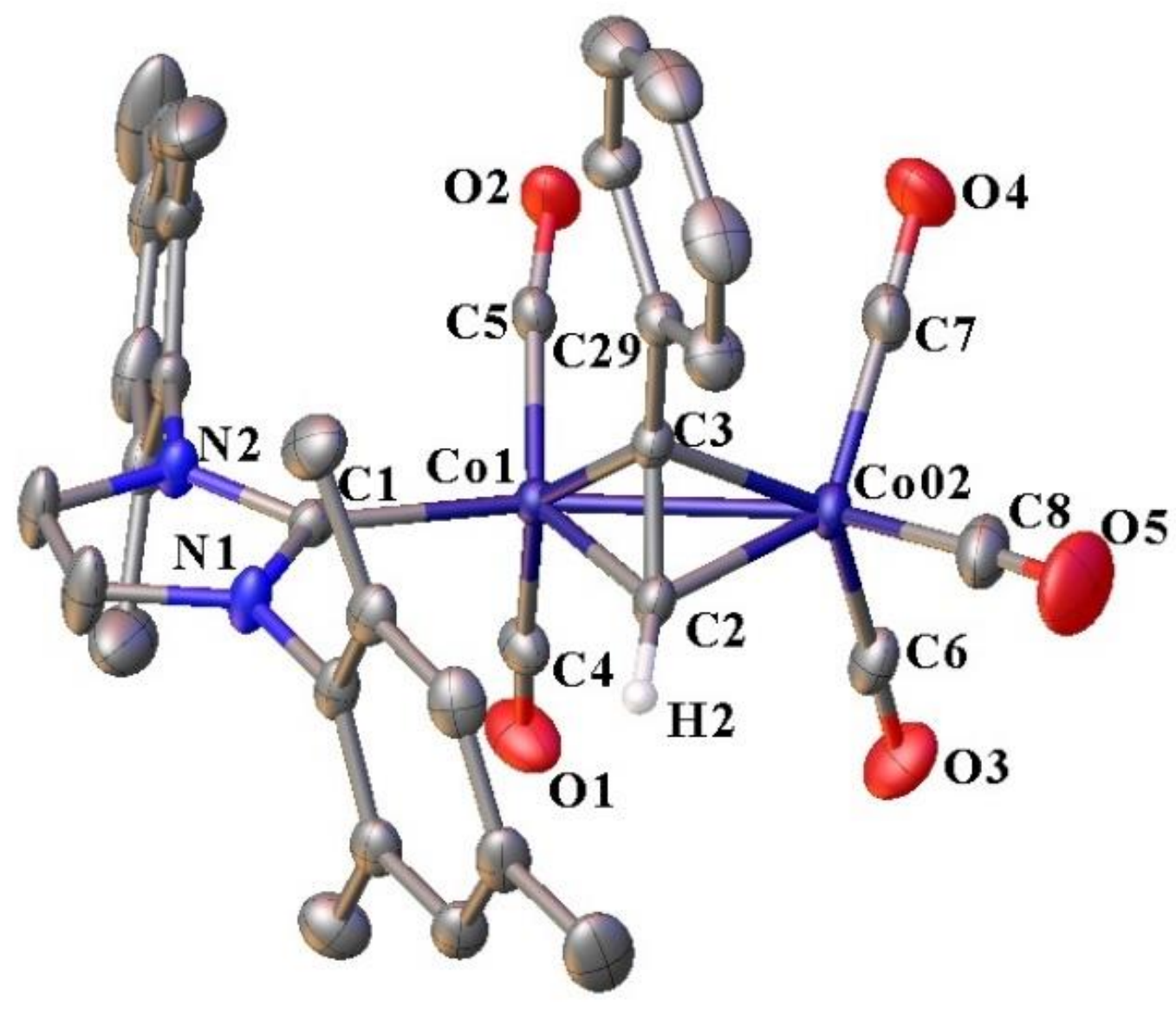

Figure S6. Molecular structure of $\mathbf{6}$ showing 30\% probability ellipsoids. Except the hydrogen atom on the bridging alkyne unit $\mathrm{CCH}$, all other hydrogen atoms were omitted for clarity. Selected bond lengths $(\AA)$ and angle $\left({ }^{\circ}\right)$ : Co1-Co02 2.4916(6), Co1-C1 1.965(2), Co1-C2 1.943(2), Co1-C3 1.938(2), Co02-C2 1.981(2), Co02-C3 2.014(2), C2-C3 1.331(3), Co1-C3-C29 141.3(1), Co02-C3-C29 127.2(1). 
Table S1. Crystal Data and Summary of Data Collection and Refinement for $\mathbf{2}, \mathbf{3}, \mathbf{4}, \mathbf{5} \cdot \mathbf{E} \mathbf{t}_{2} \mathbf{O}$ and $\mathbf{6}$

\begin{tabular}{|c|c|c|c|c|c|}
\hline Compound & 2 & 3 & 4 & $5 \cdot \mathbf{E t}_{2} \mathrm{O}$ & 6 \\
\hline Formula & $\mathrm{C}_{40} \mathrm{H}_{42} \mathrm{Co}_{2} \mathrm{~N}_{2} \mathrm{O}_{5}$ & $\mathrm{C}_{66} \mathrm{H}_{78} \mathrm{Co}_{2} \mathrm{~N}_{4} \mathrm{O}_{4}$ & $\mathrm{C}_{36} \mathrm{H}_{51} \mathrm{CoN}_{2} \mathrm{O}_{3} \mathrm{Si}$ & $\mathrm{C}_{62} \mathrm{H}_{82} \mathrm{Co}_{2} \mathrm{~N}_{4} \mathrm{O}_{5}$ & $\mathrm{C}_{34} \mathrm{H}_{30} \mathrm{Co}_{2} \mathrm{~N}_{2} \mathrm{O}_{5}$ \\
\hline Formula weight & 748.61 & 1109.18 & 646.80 & 1081.17 & 664.46 \\
\hline Temperature (K) & 173 & 170 & 170 & 170 & 193 \\
\hline Crystal system & Triclinic & Tetragonal & Orthorhombic & Monoclinic & Monoclinic \\
\hline Space group & P-1 & $\mathrm{I} 4_{1} / \mathrm{a}$ & $\mathrm{P} 212121$ & $\mathrm{P} 2{ }_{1} / \mathrm{n}$ & $\mathrm{P} 2{ }_{1} / \mathrm{c}$ \\
\hline $\mathrm{a}, \AA$ & $11.2702(8)$ & $24.7966(6)$ & $13.2589(3)$ & $14.8201(6)$ & $8.62560(10)$ \\
\hline $\mathrm{b}, \AA$ & $18.6778(14)$ & $24.7966(6)$ & $13.7103(3)$ & $19.1063(7)$ & $17.9873(2)$ \\
\hline $\mathrm{c}, \AA$ & $20.0154(14)$ & $44.4468(13)$ & $20.3237(5)$ & $20.9447(8)$ & $21.1777(3)$ \\
\hline$\alpha, \operatorname{deg}$ & $66.006(4)$ & 90 & 90 & 90 & 90 \\
\hline$\beta, \operatorname{deg}$ & $87.690(5)$ & 90 & 90 & $91.610(3)$ & $100.5090(10)$ \\
\hline$\gamma, \operatorname{deg}$ & $78.041(5)$ & 90 & 90 & 90 & 90 \\
\hline$V, \AA^{3}$ & $3761.1(5)$ & $27329.1(15)$ & $3694.51(15)$ & $5928.3(4)$ & $3230.63(7)$ \\
\hline $\mathrm{Z}$ & 4 & 16 & 4 & 4 & 4 \\
\hline$D_{\text {calcd }}, \mathrm{Mg} / \mathrm{m}^{3}$ & 1.322 & 1.078 & 1.163 & 1.211 & 1.366 \\
\hline $\operatorname{radiation}(\lambda), \AA$ & Ga K $\alpha(1.34139)$ & $\mathrm{Ga} \mathrm{K} \alpha(1.34139)$ & $\mathrm{Ga} \mathrm{K} \alpha(1.34139)$ & $\mathrm{Ga} \mathrm{K} \alpha(1.34139)$ & $\mathrm{Ga} \mathrm{K} \alpha(1.34139)$ \\
\hline $2 \theta$ range,deg & 7.0 to 110.0 & 5.6 to 110.2 & 6.8 to 109.9 & 5.4 to 110.0 & 5.6 to 109.8 \\
\hline$\mu, \mathrm{mm}^{-1}$ & 5.000 & 2.843 & 2.880 & 3.274 & 5.777 \\
\hline$F(000)$ & 1560 & 9408 & 1384 & 2304 & 1368 \\
\hline no.of obsd reflns & 14203 & 13061 & 6659 & 11274 & 6111 \\
\hline $\begin{array}{l}\text { no.of params } \\
\text { refnd }\end{array}$ & 899 & 701 & 399 & 676 & 394 \\
\hline goodness of fit & 1.059 & 1.067 & 1.086 & 1.036 & 1.057 \\
\hline $\mathrm{R} 1^{a}$ & $0.0441^{c} 0.0589^{d}$ & $0.0498^{c} 0.0911^{d}$ & $0.0336^{c} 0.0369^{d}$ & $0.0560^{c} 0.1075^{d}$ & $0.0323^{c} 0.0410^{d}$ \\
\hline$\omega \mathrm{R}_{2}^{b}$ & $0.1155^{c} 0.1229^{d}$ & $0.1132^{c} 0.1253^{d}$ & $0.0886^{c} 0.0900^{d}$ & $0.1214^{c} 0.1420^{d}$ & $0.0812^{c} 0.0851^{d}$ \\
\hline
\end{tabular}




\section{Catalyst Screening}

General procedure A: In an argon-filled glovebox, a vial $(10 \mathrm{~mL})$ was added $\mathrm{PhC} \equiv \mathrm{CH}(51 \mathrm{mg}$, $0.50 \mathrm{mmol}), \mathrm{HSiEt}_{3}(64 \mathrm{mg}, 0.55 \mathrm{mmol})$, a structurally well-defined cobalt catalyst [(IPr $\left.)_{2} \mathrm{Co}_{2}(\mathrm{CO})_{6}\right]$ (1, $5 \mathrm{~mol} \%), \quad\left[(\mathrm{IPr})(\mathrm{CO})_{2} \mathrm{Co}\left(\mu-\eta^{2}: \eta^{2}-\mathrm{HCCPh}\right) \mathrm{Co}(\mathrm{CO})_{3}\right] \quad(2, \quad 5 \quad \mathrm{~mol} \%)$, $\left[(\operatorname{IPr})(\mathrm{CO})_{2} \mathrm{Co}\left(\mu-\eta^{2}: \eta^{2}-\mathrm{HCCPh}\right) \mathrm{Co}(\mathrm{CO})_{2}(\mathrm{IPr})\right](\mathbf{3}, 5 \mathrm{~mol} \%),\left[(\mathrm{IPr}) \mathrm{Co}(\mathrm{CO})_{3}\left(\mathrm{SiEt}_{3}\right)\right] \quad$ (4, $\left.10 \mathrm{~mol} \%\right)$, $\left[(\mathrm{IPr})_{2} \mathrm{Co}_{2}(\mathrm{CO})_{4}\right](5,5 \mathrm{~mol} \%)$, or $\left[(\mathrm{IMes})(\mathrm{CO})_{2} \mathrm{Co}\left(\mu-\eta^{2}: \eta^{2}-\mathrm{HCCPh}\right) \mathrm{Co}(\mathrm{CO})_{3}\right](\mathbf{6}, 5 \mathrm{~mol} \%)$, and THF $(1 \mathrm{~mL})$. The reaction mixture was then kept stirring at $60{ }^{\circ} \mathrm{C}$ for $5 \mathrm{~h}$. After cooling to room temperature, $n$-dodecane $(85 \mathrm{mg}, 0.50 \mathrm{mmol})$ as an internal standard and $n$-hexane $(5 \mathrm{~mL})$ were added. The solution was then analyzed by GC. The results were compiled in Table 1. Selected GC graphs are shown in Figures S157-S159.

General procedure B: In an argon-filled glovebox, a vial $(10 \mathrm{~mL})$ was added $\mathrm{Co}_{2}(\mathrm{CO})_{8}(8.5 \mathrm{mg}$, $0.025 \mathrm{mmol})$, a NHC or phosphine ligand $(0.050 \mathrm{mmol})$ and THF $(1 \mathrm{~mL})$. The reaction mixture was stirred at room temperature for $2 \mathrm{~h}$, then $\mathrm{PhC} \equiv \mathrm{CH}(51 \mathrm{mg}, 0.50 \mathrm{mmol})$ and $\mathrm{HSiEt}_{3}(64 \mathrm{mg}, 0.55$ mmol) was added. The reaction mixture was then kept stirring at $60{ }^{\circ} \mathrm{C}$ for $5 \mathrm{~h}$. After cooling to room temperature, $n$-dodecane $(85 \mathrm{mg}, 0.50 \mathrm{mmol})$ as an internal standard and $n$-hexane $(5 \mathrm{~mL})$ were added. The solution was then analyzed by GC. The results were compiled in Table 1. Selected GC graphs are shown in Figures S160-S163. 


\section{Optimization of Reaction Conditions}

General procedure: In an argon-filled glovebox, a vial $(10 \mathrm{~mL})$ was charged catalytic amount of $\mathrm{Co}_{2}(\mathrm{CO})_{8}$ and IPr (two equiv. relative to $\left.\mathrm{Co}_{2}(\mathrm{CO})_{8}\right)$ and solvent $(1 \mathrm{~mL})$. The reaction mixture was stirred at room temperature for $2 \mathrm{~h}$, then $\mathrm{PhC} \equiv \mathrm{CH}(51 \mathrm{mg}, 0.50 \mathrm{mmol})$ and $\mathrm{HSiEt}_{3}(64 \mathrm{mg}, 0.55$ $\mathrm{mmol}$ ) were added. After stirring for $5 \mathrm{~h}$ at indicated temperature, $n$-dodecane $(85 \mathrm{mg}, 0.50 \mathrm{mmol})$ as an internal standard and $n$-hexane $(5 \mathrm{~mL})$ was added. The solution was then analyzed by GC. The yields of the hydrosilylation products were listed in Table S2.

Table S2. Temperature, solvent and catalytic loading screen for the hydrosilylation of $\mathrm{PhC} \equiv \mathrm{CH}$ with $\mathrm{HSiEt}_{3}$.

\begin{tabular}{|c|c|c|c|c|c|c|c|c|}
\hline \multirow[b]{2}{*}{ Entry } & \multirow[b]{2}{*}{$\mathrm{T}\left({ }^{\circ} \mathrm{C}\right)$} & \multirow{2}{*}{$\begin{array}{c}\mathrm{HSiEt}_{3} \\
\\
\text { Solvent }\end{array}$} & \multicolumn{2}{|c|}{$\begin{array}{c}\mathrm{Co}_{2}(\mathrm{CO})_{8}+2 \mathrm{IPr} \\
\mathrm{X} \mathrm{mol} \% \\
\begin{array}{c}\text { solvent, } \\
\mathrm{T}, 5 \mathrm{~h}\end{array}\end{array}$} & Ph & $\mathrm{t}_{3}+$ & & $\begin{array}{c}\mathrm{Et}_{3} \mathrm{Si} \\
\mathrm{Ph}_{\beta-(Z)}\end{array}$ \\
\hline & & & $x$ & Conv. (\%) & $\alpha$ & $\beta-(E)$ & $\beta-(Z)$ & $\begin{array}{c}\text { Selectivity } \\
\alpha / \beta-(E)\end{array}$ \\
\hline 1 & 25 & THF & 5 & 5 & 2 & 0 & 0 & -- \\
\hline 2 & 40 & THF & 5 & 18 & 10 & 1 & 0 & 10:1 \\
\hline 3 & 50 & THF & 5 & 84 & 78 & 6 & 0 & $13: 1$ \\
\hline 4 & 60 & THF & 5 & $>99$ & 95 & 5 & 0 & 19:1 \\
\hline 5 & 60 & toluene & 5 & $>99$ & 94 & 6 & 0 & $16: 1$ \\
\hline 6 & 60 & $\mathrm{CH}_{2} \mathrm{Cl}_{2}$ & 5 & $>99$ & 76 & 5 & 0 & $15: 1$ \\
\hline 7 & 60 & THF & 2 & 98 & 93 & 5 & 0 & $18: 1$ \\
\hline 8 & 60 & THF & 1 & 78 & 69 & 4 & 0 & $17: 1$ \\
\hline
\end{tabular}




\section{General Procedures for the Catalytic Hydrosilylation Reactions}

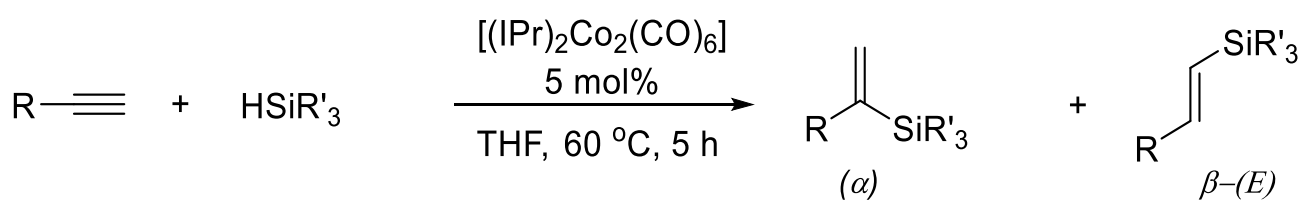

In a $\mathrm{N}_{2}$ glovebox, to a $10 \mathrm{~mL}$ screw-cap vial equipped with a stir bar was added alkyne $(2.0 \mathrm{mmol})$, hydrosilane $(2.2 \mathrm{mmol})$, cobalt catalyst $(5 \mathrm{~mol} \%)$ and THF $(4 \mathrm{~mL})$. The reaction mixture was stirred at $60{ }^{\circ} \mathrm{C}$ for $5 \mathrm{~h}$, and then cooled to room temperature. The mixture was then exposed to air and was added $n$-hexane $(2 \mathrm{~mL})$. The organic phase was subjected to vacuum to remove the volatiles. The residue was then purified by fast silica gel column chromatography. The yields of the products are tabulated in Table 1. Selected GC graphs are shown in Figures S164-S181. Characterization for the hydrosilylation products are listed as following.

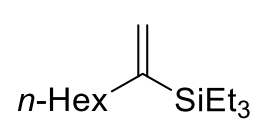

Triethyl(oct-1-en-2-yl)silane (P1) The reaction was carried out according to general procedure by using oct-1-yne (220 mg, $2.0 \mathrm{mmol}), \mathrm{HSiEt}_{3}(255 \mathrm{mg}, 2.2 \mathrm{mmol})$ and catalyst 1 (106 mg, 0.1 mmol) at $60{ }^{\circ} \mathrm{C}$ for $5 \mathrm{~h}$. Purification by fast silica gel column chromatography using petroleum ether as the eluent afforded the title compound as a colorless oil (393 mg, 87\%). ${ }^{1} \mathrm{H} \mathrm{NMR} \mathrm{(400} \mathrm{MHz}, \mathrm{CDCl}_{3}, 298$ $\mathrm{K}): \delta(\mathrm{ppm}) 5.63-5.62(\mathrm{~m}, 1 \mathrm{H}), 5.29(\mathrm{~d}, 1 \mathrm{H}, J=3.1 \mathrm{~Hz}), 2.09-2.05(\mathrm{~m}, 3 \mathrm{H}), 1.41-1.28(\mathrm{~m}, 10 \mathrm{H}), 0.92(\mathrm{t}, 9 \mathrm{H}, J=7.9$ $\mathrm{Hz}), 0.61$ (q, 6H, $J=7.9 \mathrm{~Hz}$ ). ${ }^{13} \mathrm{C} \mathrm{NMR}\left(100 \mathrm{MHz}, \mathrm{CDCl}_{3}, 298 \mathrm{~K}\right): \delta(\mathrm{ppm}) 149.4,125.1,36.6,32.0,29.5,29.1$, 22.9, 14.3, 7.5, 3.1. ${ }^{29} \mathrm{Si} \mathrm{NMR}\left(79 \mathrm{MHz}, \mathrm{CDCl}_{3}, 297 \mathrm{~K}\right): \delta$ (ppm) 1.6. These data of ${ }^{1} \mathrm{H}$ NMR and ${ }^{13} \mathrm{C}$ NMR are in accordance with the literature. ${ }^{9}$<smiles>C=C(CCC)CCCCCl</smiles>

(6-chlorohex-1-en-2-yl)triethylsilane (P2) The reaction was carried out according to general procedure by using 6-chlorohex-1-yne (232 mg, $2.0 \mathrm{mmol}), \mathrm{HSiEt}_{3}(255 \mathrm{mg}, 2.2$ $\mathrm{mmol}$ ) and catalyst $1(106 \mathrm{mg}, 0.1 \mathrm{mmol})$ at $60^{\circ} \mathrm{C}$ for $5 \mathrm{~h}$. Purification by fast silica gel column chromatography using petroleum ether as the eluent afforded the title compound as a colorless oil (394 $\mathrm{mg}$, 85\%). ${ }^{1} \mathrm{H}$ NMR (400 MHz, CDCl $\left.3,298 \mathrm{~K}\right): \delta(\mathrm{ppm}) 5.65(\mathrm{~d}, 1 \mathrm{H}, J=3.0 \mathrm{~Hz}), 5.33(\mathrm{~d}, 1 \mathrm{H}, J=3.0 \mathrm{~Hz}), 3.54(\mathrm{~m}, 2 \mathrm{H})$, 2.14-2.10 (m, 2H), 1.82-1.75 (m, 2H), 1.61-1.53 (m, 2H), $0.94(\mathrm{t}, 9 \mathrm{H}, J=7.9 \mathrm{~Hz}), 0.60(\mathrm{q}, 6 \mathrm{H}, J=7.9 \mathrm{~Hz}) .{ }^{13} \mathrm{C}$ NMR (100 MHz, $\mathrm{CDCl}_{3}, 298 \mathrm{~K}$ ): $\delta$ (ppm) 148.4, 126.0, 45.1, 35.6, 32.6, 26.3, 7.5, 3.1. HRMS (FI): calcd for $\mathrm{C}_{12} \mathrm{H}_{25} \mathrm{ClSi}[\mathrm{M}]^{+}:$232.1409; Found: 232.1412.<smiles>C=C(CC)COc1ccccc1</smiles>

Triethyl(3-phenoxyprop-1-en-2-yl)silane (P3) The reaction was carried out according to general procedure by using (prop-2-yn-1-yloxy)benzene (264 mg, $2.0 \mathrm{mmol}$ ), $\mathrm{HSiEt}_{3}$ (255 mg, $2.2 \mathrm{mmol}$ ) and catalyst $1(106 \mathrm{mg}, 0.1 \mathrm{mmol})$ at $60{ }^{\circ} \mathrm{C}$ for $5 \mathrm{~h}$. Purification by fast silica gel column chromatography using petroleum ether as the eluent afforded the title compound as a colorless oil (436 $\mathrm{mg}$, 88\%). ${ }^{1} \mathrm{H}$ NMR (400 MHz, $\left.\mathrm{CDCl}_{3}, 293 \mathrm{~K}\right): \delta(\mathrm{ppm}) 7.30-7.26(\mathrm{~m}, 2 \mathrm{H}), 6.95-6.89(\mathrm{~m}, 3 \mathrm{H}), 5.89(\mathrm{~m}, 1 \mathrm{H}), 5.49(\mathrm{dt}$, $1 \mathrm{H}, J=2.7,1.3 \mathrm{~Hz}), 4.59(\mathrm{t}, 2 \mathrm{H}, J=1.3 \mathrm{~Hz}), 0.96(\mathrm{t}, 9 \mathrm{H}, J=7.9 \mathrm{~Hz}), 0.68(\mathrm{q}, 6 \mathrm{H}, J=7.9 \mathrm{~Hz}) .{ }^{13} \mathrm{C}$ NMR $(100$ $\mathrm{MHz}, \mathrm{CDCl}_{3}, 298 \mathrm{~K}$ ): $\delta(\mathrm{ppm}) 159.0,144.7,129.5,126.9,120.7,114.8,72.3,7.4,3.1$. HRMS (EI): calcd for $\mathrm{C}_{15} \mathrm{H}_{24} \mathrm{OSi}[\mathrm{M}]^{+}:$248.1591; Found: 248.1598 .<smiles>C=C(CC)COC(=O)c1ccccc1</smiles>

2-(triethylsilyl)allylbenzoate (P4) The reaction was carried out according to general procedure by using prop-2-yn-1-ylbenzoate (320 mg, $2.0 \mathrm{mmol}), \mathrm{HSiEt}_{3}(255 \mathrm{mg}, 2.2 \mathrm{mmol})$ and catalyst $1(106 \mathrm{mg}, 0.1 \mathrm{mmol})$ at $60{ }^{\circ} \mathrm{C}$ for $5 \mathrm{~h}$. Purification by fast silica gel column 
chromatography using petroleum ether and ethyl acetate (40:1) as the eluent afforded the title compound as a colorless oil (392 mg, 71\%). ${ }^{1} \mathrm{H}$ NMR (400 MHz, $\left.\mathrm{CDCl}_{3}, 298 \mathrm{~K}\right) \delta(\mathrm{ppm})$ 8.09-8.07 (m, 2H), 7.57-7.55 (m, 1H), 7.47-7.43 (m, 2H), 5.98-5.97 (m, 1H), 5.51-5.49 (m, 1H), 4.92-4.91 (m, 2H), $0.97(\mathrm{t}, 9 \mathrm{H}, J=7.9 \mathrm{~Hz}), 0.65(\mathrm{q}, 6 \mathrm{H}, J$ $=7.9 \mathrm{~Hz}) .{ }^{13} \mathrm{C}$ NMR $\left(100 \mathrm{MHz}, \mathrm{CDCl}_{3}, 298 \mathrm{~K}\right): \delta(\mathrm{ppm}) 166.4,143.4,133.0,130.5,129.7,128.5,127.0,68.7,7.4$, 3.0. HRMS (ESI): calcd for $\mathrm{C}_{16} \mathrm{H}_{25} \mathrm{O}_{2} \mathrm{Si}[\mathrm{M}+\mathrm{H}]^{+}:$277.1618; Found: 277.1617 .

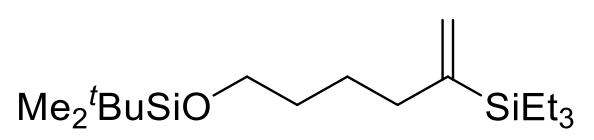

Tert-butyldimethyl((5-(triethylsilyl)hex-5-en-1-yl)oxy)silane (P5) The reaction was carried out according to general procedure by using tert-butyl(hex-5-yn-1-yloxy)dimethylsilane (424 mg, $2.0 \mathrm{mmol}$ ), $\mathrm{HSiEt}_{3}$ (255 mg, $2.2 \mathrm{mmol}$ ) and catalyst $1(106 \mathrm{mg}, 0.1 \mathrm{mmol})$ at $60{ }^{\circ} \mathrm{C}$ for $5 \mathrm{~h}$. Purification by fast silica gel column chromatography using petroleum ether as the eluent afforded the title compound as a colorless oil (472 $\mathrm{mg}, 72 \%$ ). ${ }^{1} \mathrm{H}$ NMR (400 MHz, $\left.\mathrm{CDCl}_{3}, 296 \mathrm{~K}\right): \delta(\mathrm{ppm}) 5.64-5.62(\mathrm{~m}, 1 \mathrm{H}), 5.29(\mathrm{~d}, 1 \mathrm{H}, J=3.0 \mathrm{~Hz}), 3.62(\mathrm{t}, 2 \mathrm{H}, J=6.2 \mathrm{~Hz})$, $2.10(\mathrm{~m}, 2 \mathrm{H}), 1.56-1.41(\mathrm{~m}, 4 \mathrm{H}), 0.94-0.90(\mathrm{~m}, 18 \mathrm{H}), 0.60(\mathrm{~m}, 6 \mathrm{H}), 0.05(\mathrm{~s}, 6 \mathrm{H}) .{ }^{13} \mathrm{C} \mathrm{NMR}\left(100 \mathrm{MHz}, \mathrm{CDCl}_{3}, 296\right.$ $\mathrm{K}): \delta(\mathrm{ppm}) 149.0,125.1,63.1,36.2,32.8,26.0,25.2,18.4,7.4,3.0,-5.3$. HRMS (FI): calcd for $\mathrm{C}_{18} \mathrm{H}_{40} \mathrm{OSi}_{2}[\mathrm{M}]^{+}$: 328.2612; Found: 328.2615 .<smiles>C=C(CCC)CCCCO</smiles>

5-(triethylsilyl)hex-5-en-1-ol (P6) The reaction was carried out according to general procedure by using hex-5-yn-1-ol (196 mg, $2.0 \mathrm{mmol}), \mathrm{HSiEt}_{3}(232 \mathrm{mg}, 2.0 \mathrm{mmol})$ and catalyst 1 (106 mg, $0.1 \mathrm{mmol})$ at $60{ }^{\circ} \mathrm{C}$ for $5 \mathrm{~h}$. Purification by fast silica gel column chromatography using petroleum ether and ethyl acetate (20:1) as the eluent afforded the title compound as a colorless oil (304 mg, 71\%). ${ }^{1} \mathrm{H}$ NMR (400 MHz, $\left.\mathrm{CDCl}_{3}, 298 \mathrm{~K}\right): \delta(\mathrm{ppm}) 5.64(\mathrm{~m}, 1 \mathrm{H}), 5.31(\mathrm{~d}, 1 \mathrm{H}, J=2.8 \mathrm{~Hz})$, $3.67(\mathrm{t}, 2 \mathrm{H}, J=6.4 \mathrm{~Hz}), 2.13-2.09(\mathrm{~m}, 2 \mathrm{H}), 1.62-1.55(\mathrm{~m}, 2 \mathrm{H}), 1.52-1.44(\mathrm{~m}, 2 \mathrm{H}), 1.30(\mathrm{~s}, 1 \mathrm{H}), 0.92(\mathrm{t}, 9 \mathrm{H}, J=7.9$ $\mathrm{Hz}), 0.60$ (q, 6H, $J=7.9 \mathrm{~Hz}) .{ }^{13} \mathrm{C}$ NMR (100 MHz, $\left.\mathrm{CDCl}_{3}, 295 \mathrm{~K}\right): \delta$ (ppm) 148.8, 125.3, 63.0, 36.0, 32.8, 25.0, 7.5, 3.0. HRMS (FI): calcd for $\mathrm{C}_{12} \mathrm{H}_{26} \mathrm{OSi}[\mathrm{M}]^{+}: 214.1747$; Found: 214.1752.<smiles>C=C(CC)CCO</smiles>

3-(triethylsilyl)but-3-en-1-ol (P7) The reaction was carried out according to general procedure by using but-3-yn-1-ol (140 mg, $2.0 \mathrm{mmol}), \mathrm{HSiEt}_{3}(232 \mathrm{mg}, 2.0 \mathrm{mmol})$ and catalyst $1(106 \mathrm{mg}, 0.1 \mathrm{mmol})$ at $60{ }^{\circ} \mathrm{C}$ for $5 \mathrm{~h}$. Purification by fast silica gel column chromatography using petroleum ether and DCM (2:1) as the eluent afforded the title compound as a yellow oil (294 mg, 79\%). ${ }^{1} \mathrm{H}$ NMR (400 MHz, $\left.\mathrm{CDCl}_{3}, 293 \mathrm{~K}\right): \delta(\mathrm{ppm}) 5.74(\mathrm{~m}, 1 \mathrm{H}), 5.45(\mathrm{~d}, 1 \mathrm{H}, J=2.7 \mathrm{~Hz}), 3.68(\mathrm{t}, 2 \mathrm{H}, J$ $=6.0 \mathrm{~Hz}), 2.40(\mathrm{t}, 2 \mathrm{H}, J=6.0 \mathrm{~Hz}), 1.41(\mathrm{~s}, 1 \mathrm{H}), 0.93(\mathrm{t}, 9 \mathrm{H}, J=7.9 \mathrm{~Hz}), 0.62(\mathrm{q}, 6 \mathrm{H}, J=7.9 \mathrm{~Hz}) .{ }^{13} \mathrm{C} \mathrm{NMR}(100$ $\mathrm{MHz}, \mathrm{CDCl}_{3}, 298 \mathrm{~K}$ ): $\delta(\mathrm{ppm})$ 145.5, 128.4, 61.5, 39.5, 7.4, 3.0. HRMS (FI): calcd for $\mathrm{C}_{10} \mathrm{H}_{22} \mathrm{OSi}[\mathrm{M}]^{+}: 186.1434$; Found: 186.1437.<smiles>C=C(CC)C(O)c1ccccc1</smiles>

1-phenyl-2-(triethylsilyl)prop-2-en-1-ol (P8) The reaction was carried out according to general procedure by using 1-phenylprop-2-yn-1-ol (264 mg, $2.0 \mathrm{mmol}), \mathrm{HSiEt}_{3}(255 \mathrm{mg}, 2.2$ $\mathrm{mmol})$ and catalyst $1(106.2 \mathrm{mg}, 0.1 \mathrm{mmol})$ at $60{ }^{\circ} \mathrm{C}$ for $5 \mathrm{~h}$. Purification by fast silica gel column chromatography using petroleum ether and DCM (2:1) as the eluent afforded the title compound as a yellow oil (367 mg, 74\%, $3: 1$ ). ${ }^{1} \mathrm{H}$ NMR (400 MHz, $\left.\mathrm{CDCl}_{3}, 298 \mathrm{~K}\right): \delta(\mathrm{ppm})$ 7.27-7.23 (m, 5H), 5.92 (dd, $1 \mathrm{H}, J=$ 2.5, $1.7 \mathrm{~Hz}), 5.45$, (dd, $1 \mathrm{H}, J=2.5,1.2 \mathrm{~Hz}), 5.21(\mathrm{~s}, 1 \mathrm{H}), 1.95(\mathrm{~s}, 1 \mathrm{H}), 0.75(\mathrm{t}, 9 \mathrm{H}, J=7.9 \mathrm{~Hz}), 0.33-0.45(\mathrm{~m}, 6 \mathrm{H})$. ${ }^{13} \mathrm{C}$ NMR (100 MHz, $\left.\mathrm{CDCl}_{3}, 295 \mathrm{~K}\right): \delta$ (ppm) 150.7, 142.6, 128.4, 127.8, 127.3, 125.5, 77.7, 7.3, 3.2. For $\beta$-(E) hydrosilylation product: ${ }^{1} \mathrm{H}$ NMR (400 MHz, $\left.\mathrm{CDCl}_{3}, 298 \mathrm{~K}\right): \delta(\mathrm{ppm}) 7.21-7.16(\mathrm{~m}, 5 \mathrm{H}), 6.13(\mathrm{dd}, 1 \mathrm{H}, J=18.8$, 
$5.2 \mathrm{~Hz}), 5.86(\mathrm{dd}, 1 \mathrm{H}, J=18.8,1.4 \mathrm{~Hz}), 5.08(\mathrm{~d}, 1 \mathrm{H}, \mathrm{J}=5.2 \mathrm{~Hz}), 2.11(\mathrm{~s}, 1 \mathrm{H}), 0.85(\mathrm{t}, 9 \mathrm{H}, J=7.9 \mathrm{~Hz}), 0.51(\mathrm{q}, 6 \mathrm{H}$, $J=7.9 \mathrm{~Hz}) .{ }^{13} \mathrm{C} \mathrm{NMR}\left(100 \mathrm{MHz}, \mathrm{CDCl}_{3}, 295 \mathrm{~K}\right): \delta$ (ppm) 148.6, 142.7, 128.6, 127.7, 126.6, 126.0, 77.0, 7.5, 3.5. These data are in accordance with the literature. ${ }^{10}$<smiles>C=C(CN(C)C)[SiH2]C</smiles>

N,N-dimethyl-2-(triethylsilyl)prop-2-en-1-amine (P9) The reaction was carried out according to general procedure by using N,N-dimethylprop-2-yn-1-amine (166 mg, $2.0 \mathrm{mmol}$ ), $\mathrm{HSiEt}_{3}$ $(255 \mathrm{mg}, 2.2 \mathrm{mmol})$ and catalyst $1(106 \mathrm{mg}, 0.1 \mathrm{mmol})$ at $60{ }^{\circ} \mathrm{C}$ for $5 \mathrm{~h}$. Purification by fast silica gel column chromatography using petroleum ether and ethyl acetate (5:1) as the eluent afforded the title compound as a yellow oil $(263 \mathrm{mg}, 66 \%) .{ }^{1} \mathrm{H}$ NMR $\left(400 \mathrm{MHz}, \mathrm{CDCl}_{3}, 298 \mathrm{~K}\right) \delta(\mathrm{ppm}) 5.77(\mathrm{~d}, 1 \mathrm{H}, J=1.2 \mathrm{~Hz})$, $5.38(\mathrm{~d}, 1 \mathrm{H}, J=1.2 \mathrm{~Hz}), 2.89(\mathrm{~s}, 2 \mathrm{H}), 2.14(\mathrm{~s}, 6 \mathrm{H}), 0.93(\mathrm{t}, 9 \mathrm{H}, J=7.8 \mathrm{~Hz}), 0.63(\mathrm{q}, 6 \mathrm{H}, J=7.8 \mathrm{~Hz}) .{ }^{13} \mathrm{C} \mathrm{NMR}$ $\left(100 \mathrm{MHz}, \mathrm{CDCl}_{3}, 295 \mathrm{~K}\right): \delta(\mathrm{ppm})$ 147.0, 127.1, 67.0, 45.7, 7.5, 3.0. HRMS (FI): calcd for $\mathrm{C}_{11} \mathrm{H}_{25} \mathrm{NSi}[\mathrm{M}]^{+}$: 199.1751; Found: 199.1753<smiles>C=C(CC)CCCCC(=C)[SiH2]CC</smiles>

octa-1,7-diene-2,7-diylbis(triethylsilane) (P10) The reaction was carried out according to general procedure by using octa-1,7-diyne (356 mg, $2.0 \mathrm{mmol})$, $\mathrm{HSiEt}_{3}(510 \mathrm{mg}, 4.4 \mathrm{mmol})$ and catalyst $1(106 \mathrm{mg}, 0.1 \mathrm{mmol})$ at $60{ }^{\circ} \mathrm{C}$ for $5 \mathrm{~h}$. Purification by fast silica gel column chromatography using petroleum ether as the eluent afforded the title compound as a yellow oil (608 mg, 90\%). ${ }^{1} \mathrm{H}$ NMR (400 MHz, $\left.\mathrm{CDCl}_{3}, 298 \mathrm{~K}\right) \delta(\mathrm{ppm})$ 5.64-5.63 (m, 2H), $5.29(\mathrm{~d}$, $2 \mathrm{H}, J=3.0 \mathrm{~Hz}), 2.10(\mathrm{~m}, 4 \mathrm{H}), 1.44-1.40(\mathrm{~m}, 4 \mathrm{H}), 0.85(\mathrm{t}, 18 \mathrm{H}, J=7.9 \mathrm{~Hz}), 0.52(\mathrm{q}, 12 \mathrm{H}, J=7.9 \mathrm{~Hz}) .{ }^{13} \mathrm{C}$ NMR $\left(100 \mathrm{MHz}, \mathrm{CDCl}_{3}, 298 \mathrm{~K}\right): \delta(\mathrm{ppm})$ 149.3, 125.2, 36.4, 29.0, 7.5, 3.1. HRMS (FI): calcd for $\mathrm{C}_{20} \mathrm{H}_{42} \mathrm{Si}_{2}[\mathrm{M}]^{+}$: 338.2820; Found: 338.2822.<smiles>C=C(/C=C/c1ccccc1)SCC</smiles>

(E)-triethyl(4-phenylbuta-1,3-dien-2-yl)silane (P11) The reaction was carried out according to general procedure by using (E)-but-1-en-3-yn-1-ylbenzene (256 mg, 2.0 mmol), $\mathrm{HSiEt}_{3}(255 \mathrm{mg}, 2.2 \mathrm{mmol})$ and catalyst $1(106 \mathrm{mg}, 0.1 \mathrm{mmol})$ at $60{ }^{\circ} \mathrm{C}$ for $5 \mathrm{~h}$. Purification by fast silica gel column chromatography using petroleum ether as the eluent afforded the title compound as a yellow oil $(410 \mathrm{mg}, 84 \%) .{ }^{1} \mathrm{H}$ NMR $\left(400 \mathrm{MHz}, \mathrm{CDCl}_{3}, 293 \mathrm{~K}\right) \delta(\mathrm{ppm}) 7.42(\mathrm{~d}$, $2 \mathrm{H}, J=7.4 \mathrm{~Hz}), 7.32(\mathrm{t}, 2 \mathrm{H}, J=7.4 \mathrm{~Hz}), 7.22(\mathrm{t}, 1 \mathrm{H}, J=7.4 \mathrm{~Hz}), 6.92(\mathrm{~d}, 1 \mathrm{H}, J=16.3 \mathrm{~Hz}), 6.62(\mathrm{~d}, 1 \mathrm{H}, J=16.3$ $\mathrm{Hz}), 5.96(\mathrm{~d}, 1 \mathrm{H}, \mathrm{J}=3.0 \mathrm{~Hz}), 5.48(\mathrm{~d}, 1 \mathrm{H}, J=3.0 \mathrm{~Hz}), 0.98(\mathrm{t}, 9 \mathrm{H}, J=7.8 \mathrm{~Hz}), 0.76(\mathrm{q}, 6 \mathrm{H}, J=7.8 \mathrm{~Hz}) .{ }^{13} \mathrm{C} \mathrm{NMR}$ $\left(100 \mathrm{MHz}, \mathrm{CDCl}_{3}, 293 \mathrm{~K}\right): \delta$ (ppm) 145.9, 137.9, 134.6, 129.8, 128.7, 127.4, 126.6, 126.4, 7.5, 3.6. These data are in accordance with the literature. ${ }^{11}$

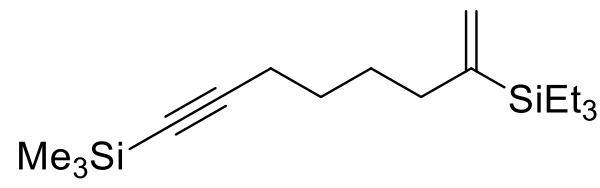

Triethyl(8-(trimethylsilyl)oct-1-en-7-yn-2-yl)silane (P12) The reaction was carried out according to general procedure by using trimethyl(octa-1,7-diyn-1-yl)silane (356 mg, $2.0 \mathrm{mmol}$ ), $\mathrm{HSiEt}_{3}(255$ $\mathrm{mg}, 2.2 \mathrm{mmol})$ and catalyst $1(106 \mathrm{mg}, 0.1 \mathrm{mmol})$ at $60{ }^{\circ} \mathrm{C}$ for $5 \mathrm{~h}$. Purification by fast silica gel column chromatography using petroleum ether as the eluent afforded the title compound as a yellow oil (482 mg, 82\%). ${ }^{1} \mathrm{H}$ NMR (400 MHz, $\left.\mathrm{CDCl}_{3}, 296 \mathrm{~K}\right) . \delta(\mathrm{ppm}) 5.63(\mathrm{dt}, 1 \mathrm{H}, J=3.0,1.6$ $\mathrm{Hz}), 5.29(\mathrm{dt}, 1 \mathrm{H}, J=3.0,1.0 \mathrm{~Hz}), 3.61(\mathrm{t}, 2 \mathrm{H}, J=6.3 \mathrm{~Hz}), 2.11-2.07(\mathrm{~m}, 2 \mathrm{H}), 1.54-1.41(\mathrm{~m}, 4 \mathrm{H}), 0.92(\mathrm{t}, 9 \mathrm{H}, J=$ $7.9 \mathrm{~Hz}), 0.59$ (q, 6H, $J=7.9 \mathrm{~Hz}), 0.05(\mathrm{~s}, 9 \mathrm{H}) .{ }^{13} \mathrm{C} \mathrm{NMR}\left(100 \mathrm{MHz}, \mathrm{CDCl}_{3}, 298 \mathrm{~K}\right): \delta(\mathrm{ppm}) 149.2,125.5,63.3$, 36.3, 32.9, 26.1, 25.3, 18.5, 7.5, 3.1, -5.1. HRMS (FI): calcd for $\mathrm{C}_{17} \mathrm{H}_{34} \mathrm{Si}_{2}[\mathrm{M}]^{+}:$294.2194; Found: 294.2198.

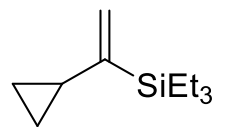

(cyclopropylvinyl)triethylsilane (P13) The reaction was carried out according to general procedure by using ethynylcyclopropane (132 mg, $2.0 \mathrm{mmol}), \mathrm{HSiEt}_{3}(255 \mathrm{mg}, 2.2 \mathrm{mmol})$ and 
catalyst $1(106 \mathrm{mg}, 0.1 \mathrm{mmol})$ at $60{ }^{\circ} \mathrm{C}$ for $5 \mathrm{~h}$. Purification by fast silica gel column chromatography using $n$-pentane as the eluent afforded the title compound as a colorless oil $(269 \mathrm{mg}, 74 \%) .{ }^{1} \mathrm{H} \mathrm{NMR}\left(400 \mathrm{MHz}, \mathrm{CDCl}_{3}\right.$, $298 \mathrm{~K}): \delta(\mathrm{ppm}) 5.29(\mathrm{dd}, 1 \mathrm{H}, J=2.6,1.3 \mathrm{~Hz}), 5.04(\mathrm{~d}, 1 \mathrm{H}, J=2.6 \mathrm{~Hz}), 1.29-1.22(\mathrm{~m}, 1 \mathrm{H}), 0.85(\mathrm{t}, 9 \mathrm{H}, J=7.8 \mathrm{~Hz})$, 0.55 (q, 6H, $J=7.8 \mathrm{~Hz}), 0.47-0.41(\mathrm{~m}, 2 \mathrm{H}), 0.35-0.31$ (m, 2H). ${ }^{13} \mathrm{C} \mathrm{NMR}\left(100 \mathrm{MHz}, \mathrm{CDCl}_{3}, 293 \mathrm{~K}\right): \delta(\mathrm{ppm})$ 150.6, 120.7, 15.5, 7.5, 7.0, 3.0. HRMS (EI): calcd for $\mathrm{C}_{11} \mathrm{H}_{22} \mathrm{Si}[\mathrm{M}]^{+}:$182.1485; Found: 182.1489.<smiles>C=C([Al])[Si]CC</smiles>

(1-cyclohexylvinyl)triethylsilane (P14) The reaction was carried out according to general procedure by using ethynylcyclohexane $(216 \mathrm{mg}, 2.0 \mathrm{mmol}), \mathrm{HSiEt}_{3}(255 \mathrm{mg}, 2.2 \mathrm{mmol})$ and catalyst $1(106 \mathrm{mg}, 0.1 \mathrm{mmol})$ at $60{ }^{\circ} \mathrm{C}$ for $5 \mathrm{~h}$. Purification by fast silica gel column chromatography using petroleum ether as the eluent afforded the title compound as a colorless oil (358 $\mathrm{mg}, 80 \%$ ). ${ }^{1} \mathrm{H}$ NMR (400 MHz, $\left.\mathrm{CDCl}_{3}, 295 \mathrm{~K}\right): \delta(\mathrm{ppm}) 5.67(\mathrm{~d}, 1 \mathrm{H}, J=2.7 \mathrm{~Hz}), 5.30(\mathrm{~d}, 1 \mathrm{H}, J=2.7 \mathrm{~Hz}), 1.99-1.94(\mathrm{~m}, 1 \mathrm{H})$, 1.77-1.66 (m, 5H), 1.31-1.07 (m, 5H), $0.92(\mathrm{t}, 9 \mathrm{H}, J=7.9 \mathrm{~Hz}), 0.59$ (q, 6H, $J=7.9 \mathrm{~Hz}) .{ }^{13} \mathrm{C}$ NMR $(100 \mathrm{MHz}$, $\mathrm{CDCl}_{3}, 295 \mathrm{~K}$ ): $\delta$ (ppm) $150.1,125.8,45.2,34.9,32.6,26.4,2.0,0.2 .{ }^{29} \mathrm{Si} \mathrm{NMR}\left(79 \mathrm{MHz}, \mathrm{CDCl}_{3}, 297 \mathrm{~K}\right): \delta(\mathrm{ppm})$ 1.9. These data of ${ }^{1} \mathrm{H}$ NMR and ${ }^{13} \mathrm{C}$ NMR are in accordance with the literature. ${ }^{12}$

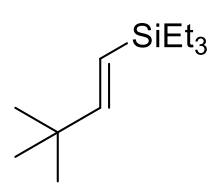

(E)-(3,3-Dimethylbut-1-en-1-yl)triethylsilane (P15) The reaction was carried out according to general procedure by using 3,3-dimethylbut-1-yne (164 mg, $2.0 \mathrm{mmol}$ ), $\mathrm{HSiEt}_{3}(255 \mathrm{mg}, 2.2 \mathrm{mmol}$ ) and catalyst 1 (106 mg, $0.1 \mathrm{mmol}$ ) at $60{ }^{\circ} \mathrm{C}$ for $5 \mathrm{~h}$. Purification by fast silica gel column chromatography using petroleum ether as the eluent afforded the title compound as a colorless oil (91 mg, 23\%). ${ }^{1} \mathrm{H}$ NMR (400 MHz, $\left.\mathrm{CDCl}_{3}, 298 \mathrm{~K}\right): \delta(\mathrm{ppm}) 6.04$ (d, $\left.1 \mathrm{H}, J=19.1 \mathrm{~Hz}\right), 5.42(\mathrm{~d}, 1 \mathrm{H}, J=19.1 \mathrm{~Hz})$, 1.00 (s, 9H), 0.92 (t, 9H, $J=7.9 \mathrm{~Hz}), 0.55$ (q, $6 \mathrm{H}, J=7.9 \mathrm{~Hz}) .{ }^{13} \mathrm{C} \mathrm{NMR}\left(100 \mathrm{MHz}, \mathrm{CDCl}_{3}, 296 \mathrm{~K}\right.$ ): $\delta$ (ppm) 159.2, $118.3,35.4,29.3,7.5,3.7$. These data are in accordance with the literature. ${ }^{13}$<smiles>C=C(CC)c1ccccc1</smiles>

Triethyl(1-phenylvinyl)silane (P16) The reaction was carried out according to general procedure by using phenylacetylene (204 mg, $2.0 \mathrm{mmol}), \mathrm{HSiEt}_{3}(255 \mathrm{mg}, 2.2 \mathrm{mmol})$ and catalyst $1(106 \mathrm{mg}, 0.1 \mathrm{mmol})$ at $60{ }^{\circ} \mathrm{C}$ for $5 \mathrm{~h}$. Purification by fast silica gel column chromatography using petroleum ether as the eluent afforded the title compound as a colorless oil (392 mg, 90\%). ${ }^{1} \mathrm{H}$ NMR (400 MHz, $\left.\mathrm{CDCl}_{3}, 296 \mathrm{~K}\right): \delta(\mathrm{ppm}) 7.32(\mathrm{t}, 2 \mathrm{H}, J=7.4 \mathrm{~Hz}), 7.24(\mathrm{t}, 1 \mathrm{H}, J=7.4 \mathrm{~Hz})$, $7.19(\mathrm{~d}, 2 \mathrm{H}, J=7.4 \mathrm{~Hz}), 5.91(\mathrm{~d}, 1 \mathrm{H}, J=3.1 \mathrm{~Hz}), 5.62(\mathrm{~d}, 1 \mathrm{H}, J=3.1 \mathrm{~Hz}), 0.97(\mathrm{t}, 9 \mathrm{H}, J=7.9 \mathrm{~Hz}), 0.71(\mathrm{q}, 6 \mathrm{H}, J=$ $7.9 \mathrm{~Hz}) .{ }^{13} \mathrm{C} \mathrm{NMR}\left(100 \mathrm{MHz}, \mathrm{CDCl}_{3}, 295 \mathrm{~K}\right): \delta$ (ppm) 150.6, 146.6, 129.0, 128.2, 126.8, 126.2, 7.4, 3.4. These data are in accordance with the literature. ${ }^{9}$

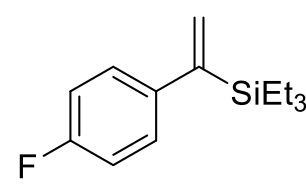

triethyl(1-(4-fluorophenyl)vinyl)silane (P17) The reaction was carried out according to general procedure by using 1-ethynyl-4-fluorobenzene ( $240 \mathrm{mg}, 2.0 \mathrm{mmol}$ ), $\mathrm{HSiEt}_{3}(255 \mathrm{mg}$, $2.2 \mathrm{mmol}$ ) and catalyst $1(106 \mathrm{mg}, 0.1 \mathrm{mmol})$ at $60^{\circ} \mathrm{C}$ for $5 \mathrm{~h}$. Purification by fast silica gel column chromatography using petroleum ether as the eluent afforded the title compound as a yellow oil (420 mg, 89\%). ${ }^{1} \mathrm{H}$ NMR (400 MHz, $\mathrm{CDCl}_{3}, 298 \mathrm{~K}$ ): $\delta$ (ppm) 7.13-7.09 (m, 2H), 7.00-6.95 (m, 2H), $5.85(\mathrm{~d}, 1 \mathrm{H}, J=2.9 \mathrm{~Hz}), 5.58(\mathrm{~d}, 1 \mathrm{H}, J=2.9 \mathrm{~Hz}), 0.93(\mathrm{t}, 9 \mathrm{H}, J=7.9 \mathrm{~Hz}), 0.66(\mathrm{q}, 6 \mathrm{H}, J=7.9 \mathrm{~Hz}) .{ }^{13} \mathrm{C}$ NMR $(100$ $\left.\mathrm{MHz}, \mathrm{CDCl}_{3}, 296 \mathrm{~K}\right): \delta(\mathrm{ppm}) 161.8(\mathrm{~d}, J=244.3 \mathrm{~Hz}), 149.6,141.5(\mathrm{~d}, J=3.3 \mathrm{~Hz}), 129.2(\mathrm{~d}, J=1.5 \mathrm{~Hz}), 128.2(\mathrm{~d}$, $J=7.8 \mathrm{~Hz}), 115.0$ (d, $J=21.2 \mathrm{~Hz}), 7.4,3.4 .{ }^{19} \mathrm{~F} \mathrm{NMR}\left(376 \mathrm{MHz}, \mathrm{CDCl}_{3}, 296 \mathrm{~K}\right): \delta(\mathrm{ppm})-117.4$. These data are in accordance with the literature. ${ }^{9}$<smiles>C=C(CC)c1ccc(Cl)cc1</smiles>

(1-(4-chlorophenyl)vinyl)triethylsilane (P18). The reaction was carried out according to general procedure by using 1-chloro-4-phenyl acetylene (272 mg, $2.0 \mathrm{mmol}$ ), $\mathrm{HSiEt}_{3}$ (255 
$\mathrm{mg}, 2.2 \mathrm{mmol})$ and catalyst $1(106 \mathrm{mg}, 0.1 \mathrm{mmol})$ at $60{ }^{\circ} \mathrm{C}$ for $5 \mathrm{~h}$. Purification by fast silica gel column chromatography using petroleum ether as the eluent afforded the title compound as a yellow oil $(448 \mathrm{mg}, 89 \%) .{ }^{1} \mathrm{H}$ NMR (400 MHz, $\left.\mathrm{CDCl}_{3}, 298 \mathrm{~K}\right): \delta(\mathrm{ppm}) 7.27(\mathrm{~d}, 2 \mathrm{H}, J=8.3 \mathrm{~Hz}), 7.09(\mathrm{~d}, 2 \mathrm{H}, J=8.3 \mathrm{~Hz}), 5.87(\mathrm{~d}, 1 \mathrm{H}, J=2.8$ $\mathrm{Hz}), 5.60(\mathrm{~d}, 1 \mathrm{H}, J=2.8 \mathrm{~Hz}), 0.94(\mathrm{t}, 9 \mathrm{H}, J=7.9 \mathrm{~Hz}), 0.67(\mathrm{q}, 6 \mathrm{H}, J=7.9 \mathrm{~Hz}) .{ }^{13} \mathrm{C} \mathrm{NMR}(100 \mathrm{MHz}, \mathrm{CDCl} 3,298$ $\mathrm{K}): \delta(\mathrm{ppm}) 149.6,144.1,132.1,129.5,128.4,128.1,7.4,3.4$. HRMS (EI): calcd for $\mathrm{C}_{14} \mathrm{H}_{21} \mathrm{ClSi}[\mathrm{M}]^{+}: 252.1096$; Found: 252.1095.<smiles>C=C(CC)c1ccc(Br)cc1</smiles>

(1-(4-bromophenyl)vinyl)triethylsilane (P19). The reaction was carried out according to general procedure by using 1-bromo-4-phenyl acetylene (362 mg, $2.0 \mathrm{mmol}$ ), $\mathrm{HSiEt}_{3}$ (255 $\mathrm{mg}, 2.2 \mathrm{mmol})$ and catalyst $1(106 \mathrm{mg}, 0.1 \mathrm{mmol})$ at $60{ }^{\circ} \mathrm{C}$ for $5 \mathrm{~h}$. Purification by fast silica gel column chromatography using petroleum ether as the eluent afforded the title compound as a yellow oil (511 mg, 86\%). ${ }^{1} \mathrm{H}$ NMR (400 MHz, $\left.\mathrm{CDCl}_{3}, 298 \mathrm{~K}\right): \delta(\mathrm{ppm}) 7.41(\mathrm{~d}, 2 \mathrm{H}, J=8.3 \mathrm{~Hz})$, $7.01(\mathrm{~d}, 2 \mathrm{H}, J=8.3 \mathrm{~Hz}), 5.85(\mathrm{~d}, 1 \mathrm{H}, J=2.9 \mathrm{~Hz}), 5.59(\mathrm{~d}, 1 \mathrm{H}, J=2.9 \mathrm{~Hz}), 0.92(\mathrm{t}, 9 \mathrm{H}, J=7.9 \mathrm{~Hz}), 0.65(\mathrm{q}, 6 \mathrm{H}, J=$ $7.9 \mathrm{~Hz}) .{ }^{13} \mathrm{C} \mathrm{NMR}\left(100 \mathrm{MHz}, \mathrm{CDCl}_{3}, 298 \mathrm{~K}\right): \delta$ (ppm) 149.6, 144.6, 131.3, 129.5, 128.5, 120.2, 7.4, 3.4. HRMS (EI): calcd for $\mathrm{C}_{14} \mathrm{H}_{21} \mathrm{BrSi}[\mathrm{M}]^{+}:$296.059; Found: 296.0601 .<smiles>C=C(CC)c1ccc(C#N)cc1</smiles>

4-(1-(triethylsilyl)vinyl)benzonitrile (P20) The reaction was carried out according to general procedure by using 4-ethynylbenzonitrile (254 mg, $2.0 \mathrm{mmol}$ ), $\mathrm{HSiEt}_{3}(255 \mathrm{mg}$, $2.2 \mathrm{mmol})$ and catalyst $1(106 \mathrm{mg}, 0.1 \mathrm{mmol})$ at $60{ }^{\circ} \mathrm{C}$ for $8 \mathrm{~h}$. Purification by fast silica gel column chromatography using petroleum ether as the eluent afforded the title compound as a yellow oil (282 mg, 58\%). ${ }^{1} \mathrm{H}$ NMR (400 MHz, $\left.\mathrm{CDCl}_{3}, 298 \mathrm{~K}\right): \delta(\mathrm{ppm}) 7.57$ (d, 2H, $J=8.3 \mathrm{~Hz}$ ), $7.21(\mathrm{~d}, 2 \mathrm{H}, J=8.3 \mathrm{~Hz}), 5.88(\mathrm{~d}, 1 \mathrm{H}, J=2.8 \mathrm{~Hz}), 5.66(\mathrm{~d}, 1 \mathrm{H}, J=2.8 \mathrm{~Hz}), 0.91(\mathrm{t}, 9 \mathrm{H}, J=7.9 \mathrm{~Hz}), 0.65(\mathrm{q}, 6 \mathrm{H}, J=$ $7.9 \mathrm{~Hz}) .{ }^{13} \mathrm{C} \mathrm{NMR}\left(100 \mathrm{MHz}, \mathrm{CDCl}_{3}, 298 \mathrm{~K}\right): \delta$ (ppm) 150.7, 149.8, 132.1, 130.8, 127.5, 119.2, 109.9, 7.3, 3.3. These data are in accordance with the literature. ${ }^{14}$<smiles>C=C(CC)c1ccc(C(F)(F)F)cc1</smiles>

Triethyl(1-(4-(trifluoromethyl)phenyl)vinyl)silane (P21) The reaction was carried out according to general procedure by using 1-trifluoromethyl-4-phenyl acetylene (340 mg, $2.0 \mathrm{mmol}), \mathrm{HSiEt}_{3}(255 \mathrm{mg}, 2.2 \mathrm{mmol})$ and catalyst $1(106 \mathrm{mg}, 0.1 \mathrm{mmol})$ at $60{ }^{\circ} \mathrm{C}$ for 5 h. Purification by fast silica gel column chromatography using petroleum ether as the eluent afforded the title compound as a yellow oil (498 mg, 87\%). ${ }^{1} \mathrm{H} \mathrm{NMR}\left(400 \mathrm{MHz}, \mathrm{CDCl}_{3}, 298 \mathrm{~K}\right): \delta(\mathrm{ppm})$ $7.54(\mathrm{~d}, 2 \mathrm{H}, J=8.0 \mathrm{~Hz}), 7.23(\mathrm{~d}, 2 \mathrm{H}, J=8.0 \mathrm{~Hz}), 5.88(\mathrm{~d}, 1 \mathrm{H}, J=2.5 \mathrm{~Hz}), 5.65(\mathrm{~d}, 1 \mathrm{H}, \mathrm{d}, J=2.5 \mathrm{~Hz}), 0.93(\mathrm{t}, 9 \mathrm{H}$, $J=7.9 \mathrm{~Hz}), 0.66$ (q, 6H, $J=7.9 \mathrm{~Hz}) \cdot{ }^{13} \mathrm{C} \mathrm{NMR}\left(100 \mathrm{MHz}, \mathrm{CDCl}_{3}, 298 \mathrm{~K}\right): \delta$ (ppm) 150.0, 149.6, 130.3, 128.5 (q, $J$ $=32.2 \mathrm{~Hz}), 127.1,125.2(\mathrm{q}, J=3.7 \mathrm{~Hz}), 124.6(\mathrm{q}, J=270.7 \mathrm{~Hz}), 7.4,3.3 .{ }^{19} \mathrm{~F}$ NMR $\left(376 \mathrm{MHz}, \mathrm{CDCl}_{3}, 298 \mathrm{~K}\right) \delta$ (ppm): -62.3. These data are in accordance with the literature. ${ }^{14}$<smiles>C=C(CC)c1ccc(C(C)=O)cc1</smiles>

Methyl-4-(1-(triethylsilyl)vinyl)benzoate (P22) The reaction was carried out according to general procedure by using methyl 4-ethynylbenzoate $(320 \mathrm{mg}, 2.0$ $\mathrm{mmol}), \mathrm{HSiEt}_{3}(255 \mathrm{mg}, 2.2 \mathrm{mmol})$ and catalyst $1(106 \mathrm{mg}, 0.1 \mathrm{mmol})$ at $60^{\circ} \mathrm{C}$ for $5 \mathrm{~h}$. Purification by fast silica gel column chromatography using petroleum ether and ethyl acetate (10: 1) as the eluent afforded the title compound as a yellow oil $(475 \mathrm{mg}, 86 \%) .{ }^{1} \mathrm{H} \mathrm{NMR}\left(400 \mathrm{MHz}, \mathrm{CDCl}_{3}\right.$, $298 \mathrm{~K}): \delta(\mathrm{ppm}) 7.96(\mathrm{~d}, 2 \mathrm{H}, J=8.2 \mathrm{~Hz}), 7.19(\mathrm{~d}, 2 \mathrm{H}, J=8.2 \mathrm{~Hz}), 5.89(\mathrm{~d}, 1 \mathrm{H}, J=2.8 \mathrm{~Hz}), 5.63(\mathrm{~d}, 1 \mathrm{H}, J=2.8$ $\mathrm{Hz}), 3.90(\mathrm{~s}, 3 \mathrm{H}), 0.91(\mathrm{t}, 9 \mathrm{H}, J=7.8 \mathrm{~Hz}), 0.66(\mathrm{q}, 6 \mathrm{H}, J=7.8 \mathrm{~Hz}) .{ }^{13} \mathrm{C} \mathrm{NMR}\left(100 \mathrm{MHz}, \mathrm{CDCl}_{3}, 298 \mathrm{~K}\right): \delta(\mathrm{ppm})$ 167.2, 150.7, 150.2, 129.9, 129.6, 128.0, 126.8, 52.1, 7.3, 3.3. ${ }^{29} \mathrm{Si} \mathrm{NMR}\left(79 \mathrm{MHz}, \mathrm{CDCl}_{3}, 297 \mathrm{~K}\right): \delta$ (ppm) 2.7. These data of ${ }^{1} \mathrm{H}$ NMR and ${ }^{13} \mathrm{C}$ NMR are in accordance with the literature. ${ }^{15}$ 
$\mathrm{MeOC}_{\mathrm{SiEt}_{3}}$

1-(4-(1-(triethylsilyl)vinyl)phenyl)ethan-1-one (P23) The reaction was carried out according to general procedure by using 1-(4-ethynylphenyl)ethan-1-one (288 mg, 2.0 $\mathrm{mmol}), \mathrm{HSiEt}_{3}(255 \mathrm{mg}, 2.2 \mathrm{mmol})$ and catalyst $1(106 \mathrm{mg}, 0.1 \mathrm{mmol})$ at $60^{\circ} \mathrm{C}$ for $5 \mathrm{~h}$. Purification by fast silica gel column chromatography using petroleum ether and ethyl acetate $(20: 1)$ as the eluent afforded the title compound as a yellow oil (369 mg, $71 \%) .{ }^{1} \mathrm{H}$ NMR (400 MHz, $\left.\mathrm{CDCl}_{3}, 298 \mathrm{~K}\right): \delta(\mathrm{ppm}) 7.89(\mathrm{~d}, 2 \mathrm{H}, J=7.9 \mathrm{~Hz}), 7.22(\mathrm{~d}, 2 \mathrm{H}, J=7.9 \mathrm{~Hz}), 5.90(\mathrm{~d}, 1 \mathrm{H}, J=2.9 \mathrm{~Hz}), 5.65(\mathrm{~d}, 1 \mathrm{H}, J$ $=2.9 \mathrm{~Hz}), 2.59(\mathrm{~s}, 3 \mathrm{H}), 0.92(\mathrm{t}, 9 \mathrm{H}, J=7.9 \mathrm{~Hz}), 0.66(\mathrm{q}, 6 \mathrm{H}, J=7.9 \mathrm{~Hz}) \cdot{ }^{13} \mathrm{C}$ NMR $\left(100 \mathrm{MHz}, \mathrm{CDCl}_{3}, 297 \mathrm{~K}\right): \delta$ (ppm) $197.8,150.9,150.1,135.1,130.0,128.4,126.9,26.6,7.3,3.3$. These data are in accordance with the literature. ${ }^{14}$<smiles>C=C(CC)c1ccc(C)cc1</smiles>

triethyl(1-(p-tolyl)vinyl)silane (P24) The reaction was carried out according to general procedure by using 1-ethynyl-4-methylbenzene (232 mg, $2.0 \mathrm{mmol}), \mathrm{HSiEt}_{3}(255 \mathrm{mg}, 2.2$ $\mathrm{mmol}$ ) and catalyst $1(106 \mathrm{mg}, 0.1 \mathrm{mmol})$ at $60^{\circ} \mathrm{C}$ for $5 \mathrm{~h}$. Purification by fast silica gel column chromatography using petroleum ether as the eluent afforded the title compound as a colorless oil (404 mg, 87\%). ${ }^{1} \mathrm{H}$ NMR (400 MHz, $\left.\mathrm{CDCl}_{3}, 298 \mathrm{~K}\right): \delta(\mathrm{ppm}) 7.10(\mathrm{~d}, 4 \mathrm{H}, J=8.0 \mathrm{~Hz}), 5.88(\mathrm{~d}, 1 \mathrm{H}$, $J=3.2 \mathrm{~Hz}), 5.56(\mathrm{~d}, 1 \mathrm{H}, J=3.2 \mathrm{~Hz}), 2.35(\mathrm{~s}, 3 \mathrm{H}), 0.95(\mathrm{t}, 9 \mathrm{H}, J=7.9 \mathrm{~Hz}), 0.69(\mathrm{q}, 6 \mathrm{H}, J=7.9 \mathrm{~Hz}) .{ }^{13} \mathrm{C} \mathrm{NMR}(100$ $\left.\mathrm{MHz}, \mathrm{CDCl}_{3}, 298 \mathrm{~K}\right): \delta(\mathrm{ppm}) 150.2,142.6,135.8,128.9,128.5,126.7,21.2,7.5,3.5$. These data are in accordance with the literature. ${ }^{16}$<smiles>C=C(CC)c1ccc(OC)cc1</smiles>

triethyl(1-(4-methoxyphenyl)vinyl)silane (P25) The reaction was carried out according to general procedure by using 1-ethynyl-4-methoxybenzene (264 mg, $2.0 \mathrm{mmol}), \mathrm{HSiEt}_{3}$ $(255 \mathrm{mg}, 2.2 \mathrm{mmol})$ and catalyst $1(106 \mathrm{mg}, 0.1 \mathrm{mmol})$ at $60{ }^{\circ} \mathrm{C}$ for $5 \mathrm{~h}$. Purification by fast silica gel column chromatography using petroleum ether as the eluent afforded the title compound as a colorless oil (431 mg, 87\%). ${ }^{1} \mathrm{H}$ NMR (400 MHz, $\left.\mathrm{CDCl}_{3}, 297 \mathrm{~K}\right): \delta(\mathrm{ppm}) 7.11(\mathrm{~d}, 2 \mathrm{H}, J=8.9$ $\mathrm{Hz}), 6.84(\mathrm{~d}, 2 \mathrm{H}, J=8.9 \mathrm{~Hz}), 5.86(\mathrm{~d}, 1 \mathrm{H}, J=3.0 \mathrm{~Hz}), 5.52(\mathrm{~d}, 1 \mathrm{H}, J=3.0 \mathrm{~Hz}), 3.80(\mathrm{~s}, 3 \mathrm{H}), 0.93(\mathrm{t}, 9 \mathrm{H}, J=7.8$ $\mathrm{Hz}), 0.67$ (q, 6H, $J=7.8 \mathrm{~Hz}) .{ }^{13} \mathrm{C}$ NMR $\left(100 \mathrm{MHz}, \mathrm{CDCl}_{3}, 297 \mathrm{~K}\right): \delta(\mathrm{ppm}) 158.3,149.5,138.0,128.1,127.8$, 113.6, 55.3, 7.5, 3.5. ${ }^{29} \mathrm{Si}$ NMR (79 MHz, $\left.\mathrm{CDCl}_{3}, 297 \mathrm{~K}\right): \delta(\mathrm{ppm}) 2.5$. These data of ${ }^{1} \mathrm{H}$ NMR and ${ }^{13} \mathrm{C}$ NMR are in accordance with the literature. ${ }^{9}$<smiles>C=C(CC)c1ccc(N(C)C)cc1</smiles>

N,N-dimethyl-4-(1-(triethylsilyl)vinyl)aniline (P26) The reaction was carried out according to general procedure by using 4-ethynyl-N,N-dimethylaniline $(290 \mathrm{mg}, 2.0$ mmol), $\mathrm{HSiEt}_{3}(255 \mathrm{mg}, 2.2 \mathrm{mmol})$ and catalyst $1(106 \mathrm{mg}, 0.1 \mathrm{mmol})$ at $60{ }^{\circ} \mathrm{C}$ for $5 \mathrm{~h}$. Purification by fast silica gel column chromatography using petroleum ether and DCM (5:1) as the eluent afforded the title compound as a yellow oil (449 mg, 86\%). ${ }^{1} \mathrm{H} \mathrm{NMR}\left(400 \mathrm{MHz}, \mathrm{CDCl}_{3}, 298 \mathrm{~K}\right)$ : $\delta(\mathrm{ppm}) 7.14(\mathrm{~d}, 2 \mathrm{H}, J=8.8 \mathrm{~Hz}), 6.74(\mathrm{~d}, 2 \mathrm{H}, J=8.8 \mathrm{~Hz}), 5.89(\mathrm{~d}, 1 \mathrm{H}, J=3.1 \mathrm{~Hz}), 5.48(\mathrm{~d}, 1 \mathrm{H}, J=3.1 \mathrm{~Hz}), 2.97(\mathrm{~s}$, $6 \mathrm{H}), 0.96(\mathrm{t}, 9 \mathrm{H}, J=7.9 \mathrm{~Hz}), 0.68(\mathrm{q}, 6 \mathrm{H}, J=7.9 \mathrm{~Hz}) .{ }^{13} \mathrm{C} \mathrm{NMR}\left(100 \mathrm{MHz}, \mathrm{CDCl}_{3}, 298 \mathrm{~K}\right): \delta(\mathrm{ppm}) 149.3,149.2$, $134.0,127.5,126.7,112.7,40.9,7.5,3.6$. These data are in accordance with the literature. ${ }^{9}$<smiles>C=C(CC)c1ccc(-c2ccccc2)cc1</smiles>

(1-([1,1'-biphenyl]-4-yl)vinyl)triethylsilane (P27) The reaction was carried out according to general procedure by using 4-ethynyl-1,1'-biphenyl (356 mg, $2.0 \mathrm{mmol}$ ), $\mathrm{HSiEt}_{3}$ (255 $\mathrm{mg}, 2.2 \mathrm{mmol})$ and catalyst $1(106 \mathrm{mg}, 0.1 \mathrm{mmol})$ at $60{ }^{\circ} \mathrm{C}$ for $5 \mathrm{~h}$. Purification by fast silica gel column chromatography using petroleum ether as the eluent afforded the title compound as a colorless oil (523 mg, 89\%). ${ }^{1} \mathrm{H}$ NMR (400 MHz, $\left.\mathrm{CDCl}_{3}, 298 \mathrm{~K}\right): \delta(\mathrm{ppm}) 7.67(\mathrm{~d}, 2 \mathrm{H}, J=7.4 \mathrm{~Hz})$, 
$7.60(\mathrm{~d}, 2 \mathrm{H}, J=8.0 \mathrm{~Hz}), 7.47(\mathrm{t}, 2 \mathrm{H}, J=7.4 \mathrm{~Hz}), 7.40-7.36(\mathrm{~m} .1 \mathrm{H}), 7.31(\mathrm{~d}, 2 \mathrm{H}, J=8.0 \mathrm{~Hz}), 6.00(\mathrm{~d}, 1 \mathrm{H}, J=2.8$ $\mathrm{Hz}), 5.67(\mathrm{~d}, 1 \mathrm{H}, J=2.8 \mathrm{~Hz}), 1.02(\mathrm{t}, 9 \mathrm{H}, J=7.8 \mathrm{~Hz}), 0.77(\mathrm{q}, 6 \mathrm{H}, J=7.8 \mathrm{~Hz}) .{ }^{13} \mathrm{C} \mathrm{NMR}(100 \mathrm{MHz}, \mathrm{CDCl}, 298$ $\mathrm{K}): \delta$ (ppm) 150.1, 144.6, 141.1, 139.1, 129.0, 128.9, 127.2, 127.2, 127.1, 127.0, 7.5, 3.5. ${ }^{29} \mathrm{Si}$ NMR (79 MHz, $\mathrm{CDCl}_{3}, 297 \mathrm{~K}$ ): $\delta$ (ppm) 2.7. HRMS (EI): calcd for $\mathrm{C}_{20} \mathrm{H}_{26} \mathrm{Si}[\mathrm{M}]^{+}: 294.1798$; Found: 294.1799.

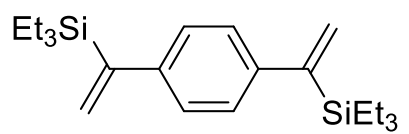

1,4-bis(1-(triethylsilyl)vinyl)benzene (P28) The reaction was carried out according to general procedure by using 1,4-diethynylbenzene ( $252 \mathrm{mg}, 2.0 \mathrm{mmol}$ ), $\mathrm{HSiEt}_{3}(510$ $\mathrm{mg}, 4.4 \mathrm{mmol}$ ) and catalyst $1(212 \mathrm{mg}, 0.2 \mathrm{mmol})$ at $60^{\circ} \mathrm{C}$ for $5 \mathrm{~h}$. Purification by fast silica gel column chromatography using petroleum ether as the eluent afforded the title compound as a colorless oil (580 mg, 81\%). ${ }^{1} \mathrm{H}$ NMR (400 MHz, $\mathrm{CDCl}_{3}, 292 \mathrm{~K}$ ): $\delta(\mathrm{ppm}) 7.09$ (s, 4H), 5.90 (d, 2H, $\left.J=3.1 \mathrm{~Hz}\right), 5.55$ (d, 2H, $J$ $=3.1 \mathrm{~Hz}), 0.92(\mathrm{t}, 18 \mathrm{H}, J=7.8 \mathrm{~Hz}), 0.67(\mathrm{q}, 12 \mathrm{H}, J=7.8 \mathrm{~Hz}) \cdot{ }^{13} \mathrm{C} \mathrm{NMR}\left(100 \mathrm{MHz}, \mathrm{CDCl}_{3}, 298 \mathrm{~K}\right): \delta(\mathrm{ppm}) 150.2$, 143.3, 128.5, 126.5, 7.5, 3.5. HRMS (FI): calcd for $\mathrm{C}_{22} \mathrm{H}_{38} \mathrm{Si}_{2}[\mathrm{M}]^{+}: 358.2507$; Found: 358.2504 .<smiles>C=Cc1ccc(C(=C)[SiH2]CC)cc1</smiles>

Triethyl(1-(4-vinylphenyl)vinyl)silane (P29) The reaction was carried out according to general procedure by using 1-ethynyl-4-vinylbenzene (256 mg, $2.0 \mathrm{mmol}$ ), $\mathrm{HSiEt}_{3}$ (255 $\mathrm{mg}, 2.2 \mathrm{mmol})$ and catalyst $1(106 \mathrm{mg}, 0.1 \mathrm{mmol})$ at $60{ }^{\circ} \mathrm{C}$ for $5 \mathrm{~h}$. Purification by fast silica gel column chromatography using petroleum ether as the eluent afforded the title compound as a cololess oil (386 mg, 79\%). ${ }^{1} \mathrm{H}$ NMR (400 MHz, $\left.\mathrm{CDCl}_{3}, 295 \mathrm{~K}\right): \delta(\mathrm{ppm}) 7.35(\mathrm{~d}, 2 \mathrm{H}, J=8.1 \mathrm{~Hz})$, $7.13(\mathrm{~d}, 2 \mathrm{H}, J=8.1 \mathrm{~Hz}), 6.71(\mathrm{dd}, 1 \mathrm{H}, J=17.6,10.9 \mathrm{~Hz}), 5.89(\mathrm{~d}, 1 \mathrm{H}, J=3.0 \mathrm{~Hz}), 5.73(\mathrm{dd}, 1 \mathrm{H}, J=17.6,0.7 \mathrm{~Hz})$, $5.57(\mathrm{~d}, 1 \mathrm{H}, J=3.0 \mathrm{~Hz}), 5.22(\mathrm{dd}, 1 \mathrm{H}, J=10.9,0.7 \mathrm{~Hz}), 0.93(\mathrm{t}, 9 \mathrm{H}, J=7.8 \mathrm{~Hz}), 0.67(\mathrm{q}, 6 \mathrm{H}, J=7.8 \mathrm{~Hz}) .{ }^{13} \mathrm{C} \mathrm{NMR}$ $\left(100 \mathrm{MHz}, \mathrm{CDCl}_{3}, 298 \mathrm{~K}\right): \delta$ (ppm) 150.1, 145.2, 136.8, 135.6, 128.9, 127.0, 126.1, 113.2, 7.5, 3.5. HRMS (EI): calcd for $\mathrm{C}_{16} \mathrm{H}_{24} \mathrm{Si}[\mathrm{M}]^{+}:$244.1642; Found: 244.1641 .<smiles>C=C(CC)c1cccc(F)c1</smiles>

Triethyl(1-(3-fluorophenyl)vinyl)silane (P30) The reaction was carried out according to general procedure by using 1-ethynyl-3-fluorobenzene ( $240 \mathrm{mg}, 2.0 \mathrm{mmol}$ ), $\mathrm{HSiEt}_{3}(255 \mathrm{mg}$, $2.2 \mathrm{mmol}$ ) and catalyst $1(106 \mathrm{mg}, 0.1 \mathrm{mmol})$ at $60{ }^{\circ} \mathrm{C}$ for $5 \mathrm{~h}$. Purification by fast silica gel column chromatography using petroleum ether as the eluent afforded the title compound as a yellow oil (382 mg, 81\%). ${ }^{1} \mathrm{H}$ NMR (400 MHz, $\left.\mathrm{CDCl}_{3}, 296 \mathrm{~K}\right): \delta(\mathrm{ppm})$ 7.27-7.21 (m, 1H), 6.93-6.83 (m, 3H), $5.88(\mathrm{~d}, 1 \mathrm{H}, J=2.9 \mathrm{~Hz}), 5.59(\mathrm{~d}, 1 \mathrm{H}, J=2.9 \mathrm{~Hz}), 0.93(\mathrm{t}, 9 \mathrm{H}, J=7.8 \mathrm{~Hz}), 0.66(\mathrm{q}, 6 \mathrm{H}, J=7.8 \mathrm{~Hz}) .{ }^{13} \mathrm{C} \mathrm{NMR}(100$ $\left.\mathrm{MHz}, \mathrm{CDCl}_{3}, 296 \mathrm{~K}\right): \delta(\mathrm{ppm}) 162.8(\mathrm{~d}, J=244.0 \mathrm{~Hz}), 149.7,148.0(\mathrm{~d}, J=7.0 \mathrm{~Hz}), 129.7,129.6,122.5(\mathrm{~d}, J=2.7$ $\mathrm{Hz}), 113.6(\mathrm{~d}, J=21.0 \mathrm{~Hz}), 113.0(\mathrm{~d}, J=21.1 \mathrm{~Hz}), 7.4,3.4 .{ }^{19} \mathrm{~F} \mathrm{NMR}\left(376 \mathrm{MHz}, \mathrm{CDCl}_{3}, 296 \mathrm{~K}\right) \delta(\mathrm{ppm}):-113.8$. ${ }^{29} \mathrm{Si} \mathrm{NMR}\left(79 \mathrm{MHz}, \mathrm{CDCl}_{3}, 297 \mathrm{~K}\right) \delta(\mathrm{ppm}):$ 2.7. HRMS (EI): calcd for $\mathrm{C}_{14} \mathrm{H}_{21} \mathrm{FSi}[\mathrm{M}]^{+}:$236.1391; Found: 236.1390 .<smiles>C=C(CC)c1cccc(Cl)c1</smiles>

(1-(3-Chlorophenyl)vinyl)triethylsilane (P31) The reaction was carried out according to general procedure by using 1-chloro-3-phenyl acetylene (272 mg, $2.0 \mathrm{mmol}$ ), $\mathrm{HSiEt}_{3}$ (255 $\mathrm{mg}, 2.2 \mathrm{mmol})$ and catalyst $1(106 \mathrm{mg}, 0.1 \mathrm{mmol})$ at $60{ }^{\circ} \mathrm{C}$ for $5 \mathrm{~h}$. Purification by fast silica gel column chromatography using petroleum ether as the eluent afforded the title compound as a yellow oil (393 mg, 78\%). ${ }^{1} \mathrm{H}$ NMR (400 MHz, $\left.\mathrm{CDCl}_{3}, 297 \mathrm{~K}\right): \delta(\mathrm{ppm})$ 7.24-7.18 (m, 2H), 7.15 (s, $1 \mathrm{H}), 7.04-7.02(\mathrm{~m}, 1 \mathrm{H}), 5.88(\mathrm{~d}, 1 \mathrm{H}, J=2.9 \mathrm{~Hz}), 5.62(\mathrm{~d}, 1 \mathrm{H}, J=2.9 \mathrm{~Hz}), 0.94(\mathrm{t}, 9 \mathrm{H}, J=7.9 \mathrm{~Hz}), 0.68(\mathrm{q}, 6 \mathrm{H}, J=$ $7.9 \mathrm{~Hz}) .{ }^{13} \mathrm{C} \mathrm{NMR}\left(100 \mathrm{MHz}, \mathrm{CDCl}_{3}, 297 \mathrm{~K}\right): \delta$ (ppm) 149.6, 147.5, 134.0, 129.8, 129.4, 126.8, 126.3, 125.0, 7.4, 3.4. HRMS (EI): calcd for $\mathrm{C}_{14} \mathrm{H}_{21} \mathrm{ClSi}[\mathrm{M}]^{+}:$252.1096; Found: 252.1095 .<smiles>C=C(CC)c1cccc(C)c1</smiles>

Triethyl(1-(m-tolyl)vinyl)silane (P32) The reaction was carried out according to general 
procedure by using1-ethynyl-3-methylbenzene (232 mg, $2.0 \mathrm{mmol}$ ), HSiEt 3 (255 mg, $2.2 \mathrm{mmol}$ ) and catalyst 1 (106 $\mathrm{mg}, 0.1 \mathrm{mmol}$ ) at $60^{\circ} \mathrm{C}$ for $5 \mathrm{~h}$. Purification by fast silica gel column chromatography using petroleum ether as the eluent afforded the title compound as a yellow oil (376 mg, 81\%). ${ }^{1} \mathrm{H}$ NMR (400 MHz, $\left.\mathrm{CDCl}_{3}, 296 \mathrm{~K}\right): \delta(\mathrm{ppm})$ 7.20-7.16 (m, 1H), 7.02-7.04 (m, 1H), 6.94-6.96 (m, 2H), $5.87(\mathrm{~d}, 1 \mathrm{H}, J=3.1 \mathrm{~Hz}), 5.56$ (d, 1H, J = 3.1 Hz), 2.35 (s, $3 \mathrm{H}$ ), 0.93 (t, 9H, $J=7.8 \mathrm{~Hz}$ ), 0.67 (q, 6H, $J=7.8 \mathrm{~Hz}) .{ }^{13} \mathrm{C} \mathrm{NMR}\left(100 \mathrm{MHz}, \mathrm{CDCl}_{3}, 296 \mathrm{~K}\right): \delta$ (ppm) 150.6, 145.6, $137.7,128.7,128.0,127.5,127.0,123.9,21.6,7.5,3.5$. These data are in accordance with the literature. ${ }^{9}$<smiles>C=C(CC)c1cccc(OC)c1</smiles>

Triethyl(1-(3-methoxyphenyl)vinyl)silane (P33) The reaction was carried out according to general procedure by using 1-ethynyl-3-methoxybenzene (264 mg, 2.0 $\mathrm{mmol}), \mathrm{HSiEt}_{3}(255 \mathrm{mg}, 2.2 \mathrm{mmol})$ and catalyst $1(106 \mathrm{mg}, 0.1 \mathrm{mmol})$ at $60^{\circ} \mathrm{C}$ for $5 \mathrm{~h}$. Purification by fast silica gel column chromatography using petroleum ether as the eluent afforded the title compound as a yellow oil (402 mg, 81\%). ${ }^{1} \mathrm{H} \mathrm{NMR}\left(400 \mathrm{MHz}, \mathrm{CDCl}_{3}, 303 \mathrm{~K}\right): \delta(\mathrm{ppm})$ 7.26-7.19 (m, 1H), 6.78-6.71 (m, 3H), 5.89 (d, $1 \mathrm{H}, J=3.0 \mathrm{~Hz}), 5.57$ (d, 1H, $J=3.0 \mathrm{~Hz}), 3.82(\mathrm{~s}, 3 \mathrm{H}), 0.94(\mathrm{t}, 9 \mathrm{H}, J$ $=7.9 \mathrm{~Hz}), 0.67$ (q, 6H, $J=7.9 \mathrm{~Hz}) .{ }^{13} \mathrm{C}$ NMR $\left(100 \mathrm{MHz}, \mathrm{CDCl}_{3}, 303 \mathrm{~K}\right): \delta$ (ppm) 159.5, 150.5, 147.1, 129.1, 129.0, 119.4, 112.6, 111.5, 55.2, 7.4, 3.4. HRMS (FI): calcd for $\mathrm{C}_{15} \mathrm{H}_{24} \mathrm{OSi}[\mathrm{M}]^{+}: 248.1591$; Found: 248.1587.

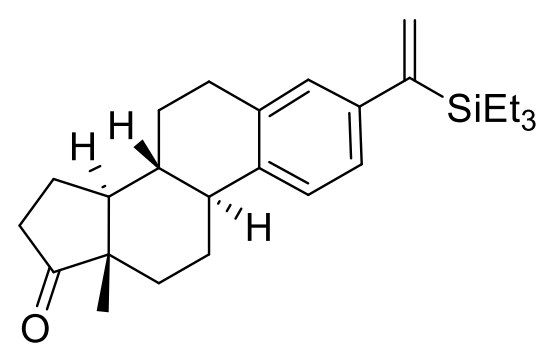

$(8 R, 9 S, 13 S, 14 S)$-13-Methyl-3-(1-(triethylsilyl)vinyl)-6,7,8,9,11,12,13,14,1 5,16-decahydro-17H-cyclopenta[a]phenanthren-17-one (P34) The reaction was carried out according to general procedure by using (8R,9S,13S,14S)-3-ethynyl-13-methyl-7,8,9,11,12,13,14,15,16-decahydro-6 H-cyclopenta[a]phenanthren-17(14H)-one (556 mg, 2.0 mmol), $\mathrm{HSiEt}_{3}(255$ $\mathrm{mg}, 2.2 \mathrm{mmol})$ and catalyst $1(106 \mathrm{mg}, 0.1 \mathrm{mmol})$ at $60{ }^{\circ} \mathrm{C}$ for $5 \mathrm{~h}$. Purification by fast silica gel column chromatography using petroleum ether, ethyl acetate and dichloromethane $(15: 1: 1)$ as the the eluent afforded the title compound as a yellow oil (646 mg, 82\%). ${ }^{1} \mathrm{H}$ NMR (400 MHz, $\left.\mathrm{CDCl}_{3}, 294 \mathrm{~K}\right): \delta(\mathrm{ppm}) 7.20(\mathrm{~d}, 1 \mathrm{H}, J=8.0 \mathrm{~Hz}), 6.95(\mathrm{dd}, 1 \mathrm{H}, J=8.0,1.7 \mathrm{~Hz})$, 6.91-6.88 (m, 1H), $5.87(\mathrm{~d}, 1 \mathrm{H}, J=3.1 \mathrm{~Hz}), 5.53(\mathrm{~d}, 1 \mathrm{H}, J=3.1 \mathrm{~Hz}), 2.90(\mathrm{dd}, 2 \mathrm{H}, J=8.6,3.9 \mathrm{~Hz}), 2.51(\mathrm{dd}, 1 \mathrm{H}, J$ $=18.8,8.5 \mathrm{~Hz}), 2.45-2.39(\mathrm{~m}, 1 \mathrm{H}), 2.35-2.26(\mathrm{~m}, 1 \mathrm{H}), 2.19-1.94(\mathrm{~m}, 4 \mathrm{H}), 1.68-1.42(\mathrm{~m}, 6 \mathrm{H}), 0.93(\mathrm{t}, 9 \mathrm{H}, J=8.0$ $\mathrm{Hz}), 0.92(\mathrm{~s}, 3 \mathrm{H}), 0.66(\mathrm{q}, 6 \mathrm{H}, J=8.0 \mathrm{~Hz}) .{ }^{13} \mathrm{C} \mathrm{NMR}\left(100 \mathrm{MHz}, \mathrm{CDCl}_{3}, 294 \mathrm{~K}\right): \delta(\mathrm{ppm}) 221.0,149.8,142.9$, 137.7, 136.1, 128.5, 127.2, 125.1, 124.3, 50.6, 48.1, 44.4, 38.3, 35.9, 31.7, 29.6, 26.6, 25.7, 21.7, 14.0, 7.5, 3.4. HRMS (EI): calcd for $\mathrm{C}_{26} \mathrm{H}_{38} \mathrm{OSi}[\mathrm{M}]^{+}: 394.2686$; Found: 394.2698 .<smiles>C=C(CC)c1ccccc1F</smiles>

Triethyl(1-(2-fluorophenyl)vinyl)silane (P35) The reaction was carried out according to general procedure by using 1-ethynyl-2-fluorobenzene ( $240 \mathrm{mg}, 2.0 \mathrm{mmol}), \mathrm{HSiEt}_{3}(255 \mathrm{mg}$, $2.2 \mathrm{mmol})$ and catalyst $1(106 \mathrm{mg}, 0.1 \mathrm{mmol})$ at $60{ }^{\circ} \mathrm{C}$ for $5 \mathrm{~h}$. Purification by fast silica gel column chromatography using petroleum ether as the eluent afforded the title compound as a yellow oil (373 mg, 79\%). ${ }^{1} \mathrm{H}$ NMR (400 MHz, $\mathrm{CDCl}_{3}, 298 \mathrm{~K}$ ): $\delta(\mathrm{ppm})$ 7.21-7.16 (m, 1H), 7.07-6.99 (m, 3H), $5.89(\mathrm{~d}, 1 \mathrm{H}, J=3.1 \mathrm{~Hz}), 5.73(\mathrm{~d}, 1 \mathrm{H}, J=3.1 \mathrm{~Hz}), 0.94(\mathrm{t}, 9 \mathrm{H}, J=7.9 \mathrm{~Hz}), 0.65(\mathrm{q}, 6 \mathrm{H}, J=7.9 \mathrm{~Hz}) .{ }^{13} \mathrm{C}$ NMR $(100$ $\left.\mathrm{MHz}, \mathrm{CDCl}_{3}, 298 \mathrm{~K}\right): \delta(\mathrm{ppm}) 159.1(\mathrm{~d}, J=243.8 \mathrm{~Hz}), 145.6,133.3(\mathrm{~d}, J=15.1 \mathrm{~Hz}), 130.9,129.7$ (d, $\left.J=4.3 \mathrm{~Hz}\right)$, $127.8(\mathrm{~d}, J=7.8 \mathrm{~Hz}), 123.9(\mathrm{~d}, J=3.5 \mathrm{~Hz}), 115.3(\mathrm{~d}, J=22.6 \mathrm{~Hz}), 7.3,3.3 .{ }^{19} \mathrm{~F} \mathrm{NMR}\left(376 \mathrm{MHz}, \mathrm{CDCl}_{3}, 298 \mathrm{~K}\right) \delta$ (ppm): -114.8. These data are in accordance with the literature. ${ }^{14}$

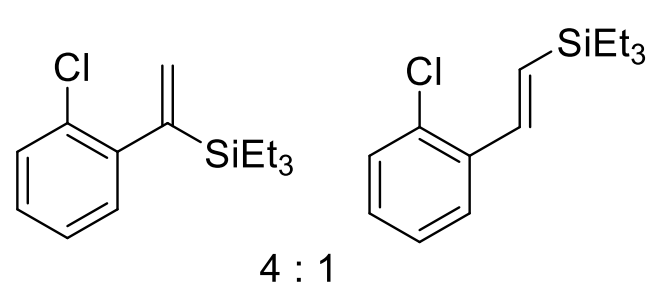

$4: 1$ (1-(2-chlorophenyl)vinyl)triethylsilane (P36) The reaction was carried out according to general procedure by using 1-ethynyl-2chlorobenzene (272 mg, $2.0 \mathrm{mmol}), \mathrm{HSiEt}_{3}(255 \mathrm{mg}, 2.2 \mathrm{mmol})$ and 
catalyst $1(106 \mathrm{mg}, 0.1 \mathrm{mmol})$ at $60{ }^{\circ} \mathrm{C}$ for $5 \mathrm{~h}$. Purification by fast silica gel column chromatography using petroleum ether as the eluent afforded the title compound as a yellow oil (403 mg, 80\%, 4: 1). ${ }^{1} \mathrm{H} \mathrm{NMR} \mathrm{(400} \mathrm{MHz,}$ $\left.\mathrm{CDCl}_{3}, 298 \mathrm{~K}\right): \delta(\mathrm{ppm}) 7.36-7.34(\mathrm{~m}, 1 \mathrm{H}), 7.24-7.11(\mathrm{~m}, 2 \mathrm{H}), 7.01-6.99(\mathrm{~m}, 1 \mathrm{H}), 5.79(\mathrm{~d}, 1 \mathrm{H}, J=3.0 \mathrm{~Hz}), 5.76(\mathrm{~d}$, $1 \mathrm{H}, J=3.0 \mathrm{~Hz}), 0.94(\mathrm{t}, 9 \mathrm{H}, J=7.9 \mathrm{~Hz}), 0.65$ (q, 6H, $J=7.9 \mathrm{~Hz}) .{ }^{13} \mathrm{C} \mathrm{NMR}\left(100 \mathrm{MHz}, \mathrm{CDCl}_{3}, 294 \mathrm{~K}\right): \delta(\mathrm{ppm})$ $149.5,144.5,140.9,130.2,129.4,129.2,127.2,126.4,7.4,3.5$. For $\beta-(E)$ hydrosilylation product: ${ }^{1} \mathrm{H}$ NMR (400 $\left.\mathrm{MHz}, \mathrm{CDCl}_{3}, 298 \mathrm{~K}\right): \delta(\mathrm{ppm}) 7.63-7.61(\mathrm{~m}, 1 \mathrm{H}), 7.33(\mathrm{~d}, 1 \mathrm{H} J=19.3 \mathrm{~Hz}), 7.19-7.11(\mathrm{~m}, 3 \mathrm{H}), 6.44(\mathrm{~d}, 1 \mathrm{H}, J=$ $19.3 \mathrm{~Hz}), 1.02$ (t, 9H, $J=7.9 \mathrm{~Hz}), 0.70$ (q, 6H, $J=7.9 \mathrm{~Hz}) .{ }^{13} \mathrm{C} \mathrm{NMR}\left(100 \mathrm{MHz}, \mathrm{CDCl}_{3}, 294 \mathrm{~K}\right): \delta(\mathrm{ppm}) 136.7$, $133.2,131.7,129.8,129.7,128.9,126.9,126.8,7.5,3.6$. These data are in accordance with the literature. ${ }^{9}$

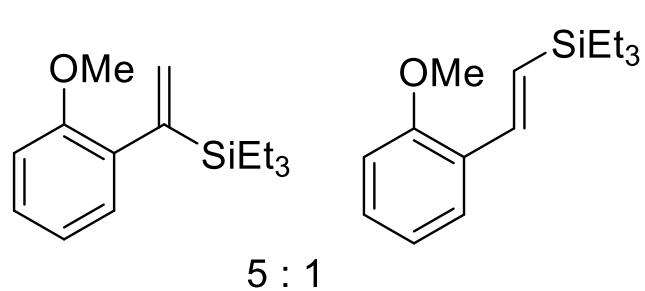

Triethyl(1-(2-methoxyphenyl)vinyl)silane (P37) The reaction was carried out according to general procedure by using 1-ethynyl-2-methoxybenzene (264 mg, $2.0 \mathrm{mmol}), \mathrm{HSiEt}_{3}(255 \mathrm{mg}$, $2.2 \mathrm{mmol})$ and catalyst $1(106 \mathrm{mg}, 0.1 \mathrm{mmol})$ at $60{ }^{\circ} \mathrm{C}$ for $5 \mathrm{~h}$. Purification by fast silica gel column chromatography using petroleum ether as the eluent afforded the title compound as a yellow oil (367 mg, 74\%, $5: 1) .{ }^{1} \mathrm{H}$ NMR (400 MHz, $\mathrm{CDCl}_{3}, 298 \mathrm{~K}$ ): $\delta$ (ppm) 7.23-7.17 (m, 1H), 6.80-6.99 (m, 3H), 5.80 $(\mathrm{d}, 1 \mathrm{H}, J=3.2 \mathrm{~Hz}), 5.61(\mathrm{~d}, 1 \mathrm{H}, J=3.2 \mathrm{~Hz}), 3.77(\mathrm{~s}, 3 \mathrm{H}), 0.90(\mathrm{t}, 9 \mathrm{H}, J=7.9 \mathrm{~Hz}), 0.60(\mathrm{q}, 6 \mathrm{H}, J=7.9 \mathrm{~Hz}) .{ }^{13} \mathrm{C}$ NMR (100 MHz, $\mathrm{CDCl}_{3}, 298 \mathrm{~K}$ ): $\delta$ (ppm) 155.8, 149.2, 135.3, 128.8, 128.5, 127.4, 120.7, 109.8, 54.8, 7.4, 3.5. For $\beta$-(E) hydrosilylation product: ${ }^{1} \mathrm{H}$ NMR $\left(400 \mathrm{MHz}, \mathrm{CDCl}_{3}, 298 \mathrm{~K}\right): \delta(\mathrm{ppm}) 7.55(\mathrm{~d}, 1 \mathrm{H}, J=7.6 \mathrm{~Hz}), 7.30(\mathrm{~d}, 1 \mathrm{H} J$ $=19.5 \mathrm{~Hz}), 7.23-7.17(\mathrm{~m}, 1 \mathrm{H}), 6.80-6.99(\mathrm{~m}, 2 \mathrm{H}), 6.38(\mathrm{~d}, 1 \mathrm{H}, J=19.5 \mathrm{~Hz}), 3.85(\mathrm{~s}, 3 \mathrm{H}), 0.99(\mathrm{t}, 9 \mathrm{H}, J=7.9 \mathrm{~Hz})$, 0.67 (q, $6 \mathrm{H}, J=7.9 \mathrm{~Hz}) .{ }^{13} \mathrm{C} \mathrm{NMR}\left(100 \mathrm{MHz}, \mathrm{CDCl}_{3}, 298 \mathrm{~K}\right): \delta(\mathrm{ppm}) 156.6,139.1,128.9,127.8,126.2,126.1$, $120.6,111.0,55.6,7.5,3.6$. These data are in accordance with the literature. ${ }^{9}$

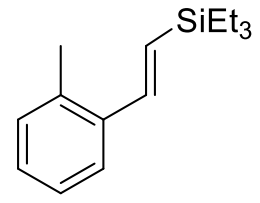

(E)-Triethyl(1-(o-tolyl)vinyl)silane (P38) The reaction was carried out according to general procedure by using 1-ethynyl-2-methylbenzene (232 mg, $2.0 \mathrm{mmol}), \mathrm{HSiEt}_{3}(255 \mathrm{mg}, 2.2 \mathrm{mmol})$ and catalyst 1 (106 mg, $0.1 \mathrm{mmol})$ at $60{ }^{\circ} \mathrm{C}$ for $5 \mathrm{~h}$. Purification by fast silica gel column chromatography using petroleum ether as the eluent afforded the title compound as a yellow oil (413 mg, 89\%). ${ }^{1} \mathrm{H}$ NMR (400 MHz, $\left.\mathrm{CDCl}_{3}, 298 \mathrm{~K}\right): \delta(\mathrm{ppm})$ 7.53-7.51 (m, 1H), 7.19-7.13 (m, 4H), 6.31 (d, 1H, $J$ $=19.2 \mathrm{~Hz}), 2.38(\mathrm{~s}, 3 \mathrm{H}), 1.00(\mathrm{t}, 9 \mathrm{H}, J=7.9 \mathrm{~Hz}), 0.67(\mathrm{q}, 6 \mathrm{H}, J=7.9 \mathrm{~Hz}) .{ }^{13} \mathrm{C} \mathrm{NMR}\left(100 \mathrm{MHz}, \mathrm{CDCl}_{3}, 298 \mathrm{~K}\right): \delta$ (ppm) 150.6, 145.6, 137.7, 128.7, 128.1, 127.5, 127.0, 123.9, 21.6, 7.5, 3.5. These data are in accordance with the literature. ${ }^{17}$

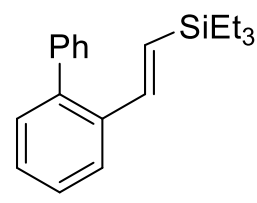

(1-([1,1'-biphenyl]-2-yl)vinyl)triethylsilane (P39) The reaction was carried out according to general procedure by using 2-ethynyl-1,1'-biphenyl (356 mg, 2.0 mmol), HSiEt $_{3}(255 \mathrm{mg}, 2.2$ mmol) and catalyst $1(106 \mathrm{mg}, 0.1 \mathrm{mmol})$ at $60{ }^{\circ} \mathrm{C}$ for $8 \mathrm{~h}$. Purification by fast silica gel column chromatography using petroleum ether as the eluent afforded the title compound as a yellow oil (265 mg, 45\%). ${ }^{1} \mathrm{H}$ NMR (400 MHz, $\left.\mathrm{CDCl}_{3}, 296 \mathrm{~K}\right): \delta(\mathrm{ppm})$ 7.94-7.90 (m, $\left.1 \mathrm{H}\right), 7.65-7.55(\mathrm{~m}, 8 \mathrm{H}), 7.17(\mathrm{~d}, 1 \mathrm{H}, J$ $=19.6 \mathrm{~Hz}), 6.62(\mathrm{~d}, 1 \mathrm{H}, J=19.6 \mathrm{~Hz}), 1.18(\mathrm{t}, 9 \mathrm{H}, J=8.0 \mathrm{~Hz}), 0.81(\mathrm{q}, 6 \mathrm{H}, J=8.0 \mathrm{~Hz}) .{ }^{13} \mathrm{C} \mathrm{NMR}(100 \mathrm{MHz}$, $\mathrm{CDCl}_{3}, 296 \mathrm{~K}$ ): $\delta$ (ppm) 144.4, 140.8, 140.7, 137.2, 130.1, 130.0, 128.0, 127.8, 127.6, 127.5, 127.1, 126.1, 7.5, 3.6. These data are in accordance with the literature. ${ }^{18}$<smiles>C=C(CC)c1ccc2cc(OC)ccc2c1</smiles>

Triethyl(1-(6-methoxynaphthalen-2-yl)vinyl)silane (P40) The reaction was carried out according to general procedure by using 2-ethynyl-6-methoxynaphthalene (364 mg, $2.0 \mathrm{mmol}), \mathrm{HSiEt}_{3}(255 \mathrm{mg}, 2.2 \mathrm{mmol})$ 
and catalyst $1(106 \mathrm{mg}, 0.1 \mathrm{mmol})$ at $60{ }^{\circ} \mathrm{C}$ for $5 \mathrm{~h}$. Purification by fast silica gel column chromatography using petroleum ether as the eluent afforded the title compound as a colorless oil $(530 \mathrm{mg}, 89 \%) .{ }^{1} \mathrm{H} \mathrm{NMR}(400 \mathrm{MHz}$, $\left.\mathrm{CDCl}_{3}, 298 \mathrm{~K}\right): \delta(\mathrm{ppm}) 7.70(\mathrm{dd}, 2 \mathrm{H}, J=15.6,8.6 \mathrm{~Hz}), 7.55(\mathrm{~s}, 1 \mathrm{H}), 7.33(\mathrm{dd}, 1 \mathrm{H}, J=8.6,1.6 \mathrm{~Hz}), 7.14-7.17(\mathrm{~m}$, 2H), $6.00(\mathrm{~d}, 1 \mathrm{H}, J=3.0 \mathrm{~Hz}), 5.66(\mathrm{~d}, 1 \mathrm{H}, J=3.0 \mathrm{~Hz}), 3.93(\mathrm{~s}, 3 \mathrm{H}), 0.97(\mathrm{t}, 9 \mathrm{H}, J=7.8 \mathrm{~Hz}), 0.73(\mathrm{q}, 6 \mathrm{H}, J=7.8$ Hz). ${ }^{13} \mathrm{C}$ NMR (100 MHz, $\mathrm{CDCl}_{3}, 298 \mathrm{~K}$ ): $\delta$ (ppm) 157.4, 150.4, 141.0, 133.3, 129.5, 129.1, 129.0, 126.5, 126.4, $124.8,118.9,105.7,55.4,7.5,3.6$. These data are in accordance with the literature. ${ }^{14}$

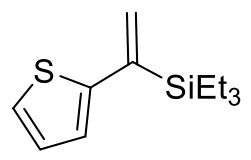

Triethyl(1-(thiophen-2-yl)vinyl)silane (P41) The reaction was carried out according to general procedure by using 2-ethynylthiophene (216 mg, $2.0 \mathrm{mmol}), \mathrm{HSiEt}_{3}(255 \mathrm{mg}, 2.2 \mathrm{mmol})$ and catalyst $1(106 \mathrm{mg}, 0.1 \mathrm{mmol})$ at $60{ }^{\circ} \mathrm{C}$ for $5 \mathrm{~h}$. Purification by fast silica gel column chromatography using petroleum ether as the eluent afforded the title compound as a orange oil $(260 \mathrm{mg}, 58 \%) .{ }^{1} \mathrm{H}$ NMR (400 MHz, $\left.\mathrm{CDCl}_{3}, 298 \mathrm{~K}\right): \delta(\mathrm{ppm}) 7.16-7.15(\mathrm{~m}, 1 \mathrm{H}), 7.00-6.94(\mathrm{~m}, 2 \mathrm{H}), 6.14(\mathrm{~d}, 1 \mathrm{H}, J=2.1 \mathrm{~Hz}), 5.50(\mathrm{~d}$, $1 \mathrm{H}, J=2.1 \mathrm{~Hz}), 0.99(\mathrm{t}, 9 \mathrm{H}, J=7.7 \mathrm{~Hz}), 0.78(\mathrm{q}, 6 \mathrm{H}, J=7.7 \mathrm{~Hz}) \cdot{ }^{13} \mathrm{C} \mathrm{NMR}\left(100 \mathrm{MHz}, \mathrm{CDCl}_{3}, 298 \mathrm{~K}\right): \delta(\mathrm{ppm})$ 147.7, 141.4, 127.6, 127.4, 124.0, 123.6, 7.5, 3.5. HRMS (EI): calcd for $\mathrm{C}_{12} \mathrm{H}_{20} \mathrm{SSi}[\mathrm{M}]^{+}$: 224.1049; Found: 224.1055 .

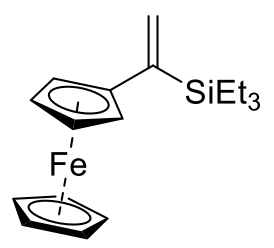

Triethyl(1-ferrocenevinyl)silane (P42) The reaction was carried out according to general procedure by using ethynylferrocene $(420 \mathrm{mg}, 2.0 \mathrm{mmol}), \mathrm{HSiEt}_{3}(255 \mathrm{mg}, 2.2 \mathrm{mmol})$ and catalyst $1(106 \mathrm{mg}, 0.1 \mathrm{mmol})$ at $60{ }^{\circ} \mathrm{C}$ for $5 \mathrm{~h}$. Purification by fast silica gel column chromatography using petroleum ether as the eluent afforded the title compound as a yellow oil $(541 \mathrm{mg}, 83 \%) .{ }^{1} \mathrm{H}$ NMR $\left(400 \mathrm{MHz}, \mathrm{CDCl}_{3}, 296 \mathrm{~K}\right): \delta(\mathrm{ppm}) 6.23(\mathrm{~d}, 1 \mathrm{H}, J=3.0 \mathrm{~Hz}), 5.51(\mathrm{~d}$, $1 \mathrm{H}, J=3.0 \mathrm{~Hz}), 4.32(\mathrm{~m}, 2 \mathrm{H}), 4.18(\mathrm{~m}, 2 \mathrm{H}), 4.07(\mathrm{~m}, 5 \mathrm{H}), 0.96(\mathrm{t}, 9 \mathrm{H}, J=7.8 \mathrm{~Hz}), 0.72(\mathrm{q}, 6 \mathrm{H}, J=7.8 \mathrm{~Hz}) .{ }^{13} \mathrm{C}$ NMR (100 MHz, $\left.\mathrm{CDCl}_{3}, 296 \mathrm{~K}\right): \delta$ (ppm) 145.0, 126.0, 88.5, 69.5, 67.8, 67.6, 7.6, 3.7. HRMS (ESI): calcd for $\mathrm{C}_{18} \mathrm{H}_{26} \mathrm{FeSi}[\mathrm{M}]^{+}:$326.11477; Found: 326.11508 .

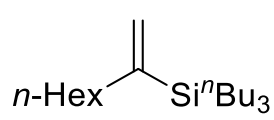

Tributyl(oct-1-en-2-yl)silane (P43) The reaction was carried out according to general procedure by using oct-1-yne (220 mg, $2.0 \mathrm{mmol}), \mathrm{HSi}^{n} \mathrm{Bu}_{3}(440 \mathrm{mg}, 2.2 \mathrm{mmol})$ and catalyst 1 (106 mg, $0.1 \mathrm{mmol}$ ) at $60{ }^{\circ} \mathrm{C}$ for $5 \mathrm{~h}$. Purification by fast silica gel column chromatography using petroleum ether as the eluent afforded the title compound as a colorless oil (521 mg, 84\%). ${ }^{1} \mathrm{H}$ NMR (400 $\left.\mathrm{MHz}, \mathrm{CDCl}_{3}, 294 \mathrm{~K}\right) \delta(\mathrm{ppm}) 5.60(\mathrm{dt}, 1 \mathrm{H}, J=3.0,1.5 \mathrm{~Hz}), 5.27(\mathrm{~d}, 1 \mathrm{H}, J=3.0 \mathrm{~Hz}), 2.09-2.05(\mathrm{~m}, 2 \mathrm{H}), 1.35-1.20$ (m, 20H), 0.90-0.87 (m, 12H), 0.61-0.57 (m, 6H). ${ }^{13} \mathrm{C} \mathrm{NMR} \mathrm{(100} \mathrm{MHz,} \mathrm{CDCl}_{3}, 298 \mathrm{~K}$ ): $\delta$ (ppm) 150.2, 124.8, 36.4, $32.0,29.4,29.0,27.0,26.2,22.8,14.3,14.0,12.0$. HRMS (FI): calcd for $\mathrm{C}_{20} \mathrm{H}_{42} \mathrm{Si}[\mathrm{M}]^{+}: 310.3050$; Found: 310.3042 .

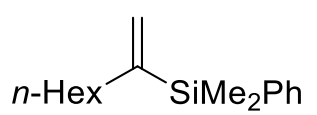

Dimethyl(oct-1-en-2-yl) (phenyl)silane (P44) The reaction was carried out according to general procedure by using oct-1-yne (220 mg, $2.0 \mathrm{mmol}), \mathrm{HSiMe}_{2} \mathrm{Ph}(299 \mathrm{mg}, 2.2 \mathrm{mmol})$ and catalyst 1 (106 mg, $0.1 \mathrm{mmol})$ at $60{ }^{\circ} \mathrm{C}$ for $5 \mathrm{~h}$. Purification by fast silica gel column chromatography using petroleum ether as the eluent afforded the title compound as a colorless oil (423 $\mathrm{mg}, 86 \%$ ). ${ }^{1} \mathrm{H}$ NMR (400 MHz, $\left.\mathrm{CDCl}_{3}, 298 \mathrm{~K}\right) \delta(\mathrm{ppm}) 7.56-7.53(\mathrm{~m}, 2 \mathrm{H}), 7.37-7.38(\mathrm{~m}, 3 \mathrm{H}), 5.71(\mathrm{~m}, 1 \mathrm{H}), 5.43(\mathrm{~d}, 1 \mathrm{H}, J=$ $3.0 \mathrm{~Hz}$ ), 2.14 (t, 2H), 1.39-1.24 (m, 8H), 0.89 (t, 3H), 0.40 (s, 6H). $\left.{ }^{13} \mathrm{C} \mathrm{NMR} \mathrm{(100} \mathrm{MHz,} \mathrm{CDCl}_{3}, 298 \mathrm{~K}\right):(\delta, \mathrm{ppm})$ $150.7,138.7,134.1,129.0,127.8,125.8,36.2,31.9,29.2,29.0,22.8,13.2,-2.7$. These data are in accordance with the literature. ${ }^{19}$

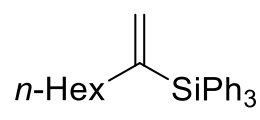

Oct-1-en-2-yltriphenylsilane (P45) The reaction was carried out according to general 
procedure by using oct-1-yne (220 mg, $2.0 \mathrm{mmol}), \mathrm{HSiPh}_{3}(572 \mathrm{mg}, 2.2 \mathrm{mmol})$ and catalyst $1(106 \mathrm{mg}, 0.1 \mathrm{mmol})$ at $60{ }^{\circ} \mathrm{C}$ for $5 \mathrm{~h}$. Purification by fast silica gel column chromatography using petroleum ether as the eluent afforded the title compound as a colorless oil (585 mg, 79\%). ${ }^{1} \mathrm{H}$ NMR $\left(400 \mathrm{MHz}, \mathrm{CDCl}_{3}, 293 \mathrm{~K}\right) \delta$ (ppm) 7.57-7.55 (m, $6 \mathrm{H}), 7.43-7.34(\mathrm{~m}, 9 \mathrm{H}), 5.99(\mathrm{dt}, 1 \mathrm{H}, J=2.9,1.5 \mathrm{~Hz}), 5.53(\mathrm{~d}, 1 \mathrm{H}, J=2.9 \mathrm{~Hz}), 2.22(\mathrm{t}, 2 \mathrm{H}, J=7.6), 1.37-1.30(\mathrm{~m}$, $2 \mathrm{H}), 1.22-1.10(\mathrm{~m}, 6 \mathrm{H}), 0.83(\mathrm{t}, 3 \mathrm{H}, J=7.0 \mathrm{~Hz}) .{ }^{13} \mathrm{C} \mathrm{NMR}\left(100 \mathrm{MHz}, \mathrm{CDCl}_{3}, 294 \mathrm{~K}\right): \delta(\mathrm{ppm}) 146.8,136.4,134.4$, $130.3,129.5,127.9,36.4,31.8,29.1,28.9,22.7,14.2$. These data are in accordance with the literature. ${ }^{20}$

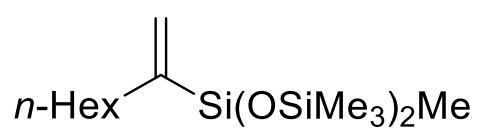

1,1,1,3,5,5,5-heptamethyl-3-(oct-1-en-2-yl)trisiloxane (P46) The reaction was carried out according to general procedure by using oct-1-yne (220 mg, 2.0 mmol), HSiMe(OTMS $)_{2}(488 \mathrm{mg}, 2.2 \mathrm{mmol})$ and catalyst 1 (106 mg, $\left.0.1 \mathrm{mmol}\right)$ at $60{ }^{\circ} \mathrm{C}$ for $5 \mathrm{~h}$. Purification by fast silica gel column chromatography using petroleum ether as the eluent afforded the title compound as a yellow oil $(571 \mathrm{mg}, 86 \%) .{ }^{1} \mathrm{H}$ NMR $\left(400 \mathrm{MHz}, \mathrm{CDCl}_{3}, 298 \mathrm{~K}\right) \delta(\mathrm{ppm}) 5.54(\mathrm{~m}, 1 \mathrm{H}), 5.40$ $(\mathrm{d}, 1 \mathrm{H}, J=3.2 \mathrm{~Hz}), 2.12-2.08(\mathrm{~m}, 2 \mathrm{H}), 1.45-1.25(\mathrm{~m}, 8 \mathrm{H}), 0.91-0.87(\mathrm{~m}, 3 \mathrm{H}), 0.10(\mathrm{~s}, 18 \mathrm{H}), 0.09(\mathrm{~s}, 3 \mathrm{H}) .{ }^{13} \mathrm{C}$ NMR $\left(100 \mathrm{MHz}, \mathrm{CDCl}_{3}, 295 \mathrm{~K}\right)$ : ( $\delta$, ppm) 151.1, 125.2, 36.0, 32.0, 29.5, 29.2, 22.9, 14.3, 2.0, -0.1. HRMS (FI): calcd for $\mathrm{C}_{15} \mathrm{H}_{36} \mathrm{O}_{2} \mathrm{Si}_{3}[\mathrm{M}]^{+}:$332.2018; Found: 332.2014.

$n$-Hex ${ }_{\mathrm{Si}(\mathrm{OEt})_{3}}$

triethoxy(oct-1-en-2-yl)silane (P47) The reaction was carried out according to general procedure by using oct-1-yne $(220 \mathrm{mg}, 2.0 \mathrm{mmol}), \mathrm{HSi}(\mathrm{OEt})_{3}(361 \mathrm{mg}, 2.2 \mathrm{mmol})$ and catalyst $1(106 \mathrm{mg}, 0.1 \mathrm{mmol})$ at $60{ }^{\circ} \mathrm{C}$ for $5 \mathrm{~h}$. Purification by fast silica gel column chromatography using petroleum ether and DCM $(5: 1)$ as the eluent afforded the title compound as a colorless oil (416 mg, 76\%). ${ }^{1} \mathrm{H}$ NMR (400 MHz, $\left.\mathrm{CDCl}_{3}, 293 \mathrm{~K}\right) \delta(\mathrm{ppm}) 5.73-5.70(\mathrm{~m}, 1 \mathrm{H}), 5.62(\mathrm{~d}, 1 \mathrm{H}, J=3.2 \mathrm{~Hz}), 3.82(\mathrm{q}$, $6 \mathrm{H}, J=7.0 \mathrm{~Hz}), 2.15-2.11(\mathrm{~m}, 3 \mathrm{H}), 1.48-1.41(\mathrm{~m}, 4 \mathrm{H}), 1.23(\mathrm{t}, 9 \mathrm{H}, J=7.0 \mathrm{~Hz}), 0.88(\mathrm{t}, 6 \mathrm{H}, \mathrm{J}=7.0 \mathrm{~Hz}) .{ }^{13} \mathrm{C} \mathrm{NMR}$ $\left(100 \mathrm{MHz}, \mathrm{CDCl}_{3}, 298 \mathrm{~K}\right): \delta$ (ppm) 144.0, 129.1, 58.6, 36.2, 31.9, 29.3, 28.9, 22.8, 18.4, 14.2. These data are in accordance with the literature. ${ }^{21}$

Tributyl(1-phenylvinyl)silane (P48) The reaction was carried out according to general procedure by using phenylacetylene (204 mg, $2.0 \mathrm{mmol}), \mathrm{HSi}^{n} \mathrm{Bu}_{3}(440 \mathrm{mg}, 2.2 \mathrm{mmol})$ and catalyst 1 (106 $\mathrm{mg}, 0.1 \mathrm{mmol})$ at $60{ }^{\circ} \mathrm{C}$ for $5 \mathrm{~h}$. Purification by fast silica gel column chromatography using petroleum ether as the eluent afforded the title compound as a yellow oil $(489 \mathrm{mg}, 81 \%) .{ }^{1} \mathrm{H}$ NMR $(400 \mathrm{MHz}$, $\left.\mathrm{CDCl}_{3}, 296 \mathrm{~K}\right) \delta(\mathrm{ppm}) 7.36-7.19(\mathrm{~m}, 5 \mathrm{H}), 5.91(\mathrm{~d}, 1 \mathrm{H}, J=3.0 \mathrm{~Hz}), 5.63(\mathrm{~d}, 1 \mathrm{H}, J=3.0 \mathrm{~Hz}), 1.42-1.28(\mathrm{~m}, 12 \mathrm{H})$, 0.94-0.90 (m, 9H), 0.74-0.70 (m, 6H). $\left.{ }^{13} \mathrm{C} \mathrm{NMR} \mathrm{(100} \mathrm{MHz,} \mathrm{CDCl}_{3}, 296 \mathrm{~K}\right): \delta$ (ppm) 151.4, 145.7, 128.6, 128.2, 126.8, 126.2, 26.8, 26.1, 13.9, 12.2. HRMS (EI): calcd for $\mathrm{C}_{20} \mathrm{H}_{34} \mathrm{Si}[\mathrm{M}]^{+}: 302.2424$; Found: 302.2428 .

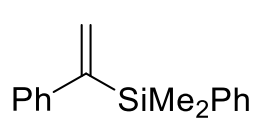

Dimethyl(phenyl)(1-phenylvinyl)silane (P49) The reaction was carried out according to general procedure by using phenylacetylene $(204 \mathrm{mg}, 2.0 \mathrm{mmol}), \mathrm{HSiMe}_{2} \mathrm{Ph}(299 \mathrm{mg}, 2.2$ $\mathrm{mmol})$ and catalyst $1(106 \mathrm{mg}, 0.1 \mathrm{mmol})$ at $60{ }^{\circ} \mathrm{C}$ for $5 \mathrm{~h}$. Purification by fast silica gel column chromatography using petroleum ether as the eluent afforded the title compound as a colorless oil (414 mg, 87\%). ${ }^{1} \mathrm{H}$ NMR $\left(400 \mathrm{MHz}, \mathrm{CDCl}_{3}, 296 \mathrm{~K}\right) \delta(\mathrm{ppm})$ 7.63-7.61 (m, 2H), 7.44-7.41 (m, 3H), 7.32-7.24 (m, 3H), 7.19-7.18 $(\mathrm{m}, 2 \mathrm{H}), 6.05(\mathrm{~d}, 1 \mathrm{H}, J=2.9 \mathrm{~Hz}), 5.74(\mathrm{~d}, 1 \mathrm{H}, J=2.9 \mathrm{~Hz}), 0.48(\mathrm{~s}, 6 \mathrm{H}) .{ }^{13} \mathrm{C} \mathrm{NMR}\left(100 \mathrm{MHz}, \mathrm{CDCl}_{3}, 296 \mathrm{~K}\right): \delta$ (ppm) 151.2, 144.3, 138.4, 134.2, 129.3, 129.2, 128.2, 128.0, 127.0, 126.5, -2.2. ${ }^{29} \mathrm{Si} \mathrm{NMR} \mathrm{(79} \mathrm{MHz,} \mathrm{CDCl}_{3}, 297$ $\mathrm{K}): \delta(\mathrm{ppm})$-8.2. These data of ${ }^{1} \mathrm{H}$ NMR and ${ }^{13} \mathrm{C}$ NMR are in accordance with the literature. ${ }^{22}$ The data of ${ }^{29} \mathrm{Si}$ NMR is in accordance with the literature..$^{27}$

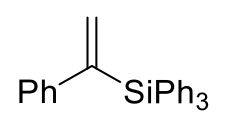

Triphenyl(1-phenylvinyl)silane (P50) The reaction was carried out according to general 
procedure by using phenylacetylene (204 mg, $2.0 \mathrm{mmol}), \mathrm{HSiPh}_{3}(572 \mathrm{mg}, 2.2 \mathrm{mmol})$ and catalyst 1 (106 mg, 0.1 $\mathrm{mmol}$ ) at $60^{\circ} \mathrm{C}$ for $5 \mathrm{~h}$. Purification by fast silica gel column chromatography using petroleum ether as the eluent afforded the title compound as a colorless solid $(594 \mathrm{mg}, 82 \%)$. Melting point: 102.6-104.3 ${ }^{\circ} \mathrm{C} .{ }^{1} \mathrm{H} \mathrm{NMR}(400 \mathrm{MHz}$, $\left.\mathrm{CDCl}_{3}, 295 \mathrm{~K}\right) \delta(\mathrm{ppm})$ 7.55-7.52 (m, 6H), 7.44-7.40 (m, 4H), 7.37-7.33 (m, 6H), 7.20-7.19 (m, 4H), 6.31 (d, 1H, $J$ $=2.7 \mathrm{~Hz}), 5.71(\mathrm{~d}, 1 \mathrm{H}, J=2.7 \mathrm{~Hz}) .{ }^{13} \mathrm{C}$ NMR $\left(100 \mathrm{MHz}, \mathrm{CDCl}_{3}, 295 \mathrm{~K}\right): \delta(\mathrm{ppm}) 147.4,144.0,136.5,134.3,134.1$, 129.7, 128.3, 128.0, 127.6, 126.9. ${ }^{29} \mathrm{Si} \mathrm{NMR}\left(79 \mathrm{MHz}, \mathrm{CDCl}_{3}, 297 \mathrm{~K}\right): \delta(\mathrm{ppm})-14.6$. These data of ${ }^{1} \mathrm{H}$ NMR and

${ }^{13} \mathrm{C}$ NMR are in accordance with the literature. ${ }^{23}$ The data of ${ }^{29} \mathrm{Si}$ NMR is in accordance with the literature. ${ }^{27}$<smiles>C=C([SiH2]OCCCC)c1ccccc1</smiles>

1,1,1,3,5,5,5-Heptamethyl-3-(1-phenylvinyl)trisiloxane (P51) The reaction was carried out according to general procedure by using phenylacetylene $(204 \mathrm{mg}, 2.0$ $\mathrm{mmol}), \mathrm{HSiMe}(\mathrm{OTMS})_{2}(488 \mathrm{mg}, 2.2 \mathrm{mmol})$ and catalyst $1(106 \mathrm{mg}, 0.1 \mathrm{mmol})$ at $60{ }^{\circ} \mathrm{C}$ for $5 \mathrm{~h}$. Purification by fast silica gel column chromatography using petroleum ether as the eluent afforded the title compound as a yellow oil $(557 \mathrm{mg}, 86 \%) .{ }^{1} \mathrm{H} \mathrm{NMR}\left(400 \mathrm{MHz}, \mathrm{CDCl}_{3}, 298 \mathrm{~K}\right) \delta(\mathrm{ppm}):$ 7.37-7.22 (m, 5H), $5.93(\mathrm{~d}$, $1 \mathrm{H}, J=3.0 \mathrm{~Hz}), 5.73(\mathrm{~d}, 1 \mathrm{H}, J=3.0 \mathrm{~Hz}), 0.22(\mathrm{~s}, 3 \mathrm{H}), 0.08(\mathrm{~s}, 18 \mathrm{H}) .{ }^{13} \mathrm{C} \mathrm{NMR}\left(100 \mathrm{MHz}, \mathrm{CDCl}_{3}, 295 \mathrm{~K}\right):(\delta, \mathrm{ppm})$ 150.9, 143.1, 128.2, 128.0, 127.1, 126.7, 1.9, 0.3. HRMS (EI): calcd for $\mathrm{C}_{15} \mathrm{H}_{28} \mathrm{O}_{2} \mathrm{Si}_{3}[\mathrm{M}]^{+}:$324.1392; Found: 324.1405 .<smiles>C=C(c1ccccc1)[SiH](CC)OCC</smiles>

Triethoxy(1-phenylvinyl)silane (P52) The reaction was carried out according to general procedure by using phenylacetylene $(204 \mathrm{mg}, 2.0 \mathrm{mmol}), \mathrm{HSi}(\mathrm{OEt})_{3}(361 \mathrm{mg}, 2.2 \mathrm{mmol})$ and catalyst $1(106 \mathrm{mg}, 0.1 \mathrm{mmol})$ at $60{ }^{\circ} \mathrm{C}$ for $5 \mathrm{~h}$. Purification by fast silica gel column chromatography using petroleum ether and DCM $(5: 1)$ as the eluent afforded the title compound as a colorless oil (388 mg, 73\%). ${ }^{1} \mathrm{H}$ NMR (400 MHz, $\left.\mathrm{CDCl}_{3}, 298 \mathrm{~K}\right) \delta(\mathrm{ppm}) 7.46-7.44(\mathrm{~m}, 2 \mathrm{H}), 7.32-7.21(\mathrm{~m}, 3 \mathrm{H}), 6.15(\mathrm{~d}, 1 \mathrm{H}, J$ $=3.0 \mathrm{~Hz}), 5.97(\mathrm{~d}, 1 \mathrm{H}, J=3.0 \mathrm{~Hz}), 3.83(\mathrm{q}, 6 \mathrm{H}, J=7.0 \mathrm{~Hz}), 1.20(\mathrm{t}, 9 \mathrm{H}, J=7.0 \mathrm{~Hz}) .{ }^{13} \mathrm{C} \mathrm{NMR}\left(100 \mathrm{MHz}, \mathrm{CDCl}_{3}\right.$, $298 \mathrm{~K}): \delta(\mathrm{ppm}) 143.4,142.1,131.8,128.4,127.1,127.0,58.9,18.3$. These data are in accordance with the literature. ${ }^{24}$

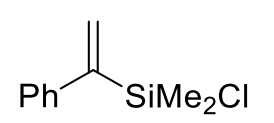

Chlorodimethyl(1-phenylvinyl)silane (P53) The reaction was carried out according to general procedure by using phenylacetylene $(510 \mathrm{mg}, 5.0 \mathrm{mmol}), \mathrm{HSiMe}_{2} \mathrm{Cl}(517 \mathrm{mg}, 5.5 \mathrm{mmol})$ and catalyst $1(265 \mathrm{mg}, 0.25 \mathrm{mmol})$ at $60^{\circ} \mathrm{C}$ for $5 \mathrm{~h}$. Purification by distillation and give colorless oil (421 mg, 43\%). ${ }^{1} \mathrm{H}$ NMR (400 MHz, $\left.\mathrm{C}_{6} \mathrm{D}_{6}, 294 \mathrm{~K}\right) \delta(\mathrm{ppm})$ 7.32-7.30 (m, 2H), 7.18-7.14 (m, 2H), 7.09-7.11 (m, $1 \mathrm{H}), 5.84(\mathrm{~d}, 1 \mathrm{H}, J=2.3 \mathrm{~Hz}), 5.70(\mathrm{~d}, 1 \mathrm{H}, J=2.3 \mathrm{~Hz}), 0.36(\mathrm{~s}, 6 \mathrm{H}) .{ }^{13} \mathrm{C} \mathrm{NMR}\left(100 \mathrm{MHz}, \mathrm{C}_{6} \mathrm{D}_{6}, 298 \mathrm{~K}\right): \delta(\mathrm{ppm})$ 149.8, 142.6, 130.3, 128.7, 127.4, 127.3, 2.0. HRMS (FI): calcd for $\mathrm{C}_{10} \mathrm{H}_{13} \mathrm{ClSi}[\mathrm{M}]^{+}$: 196.0470; Found: 196.0472.

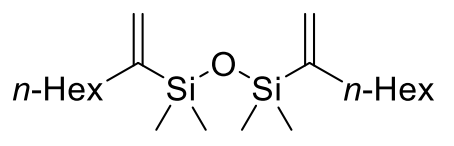

2,2'-(1,1,3,3-Tetramethyldisiloxane-1,3-diyl)bis(oct-1-en-3-one) (P54) The reaction was carried out according to general procedure by using oct-1-yne (220 $\mathrm{mg}, 2.0 \mathrm{mmol}),\left(\mathrm{HSiMe}_{2}\right)_{2} \mathrm{O}(134 \mathrm{mg}, 1 \mathrm{mmol})$ and catalyst $1(106 \mathrm{mg}, 0.1 \mathrm{mmol})$ at $60{ }^{\circ} \mathrm{C}$ for $5 \mathrm{~h}$. Purification by fast silica gel column chromatography using petroleum ether as the eluent afforded the title compound as a colorless oil $(283 \mathrm{mg}, 80 \%) .{ }^{1} \mathrm{H}$ NMR $\left(400 \mathrm{MHz}, \mathrm{CDCl}_{3}, 298 \mathrm{~K}\right) \delta(\mathrm{ppm}) 5.56(\mathrm{~m}, 2 \mathrm{H})$, $5.38(\mathrm{~d}, 2 \mathrm{H}, J=3.0 \mathrm{~Hz}), 2.16-2.13(\mathrm{~m}, 4 \mathrm{H}), 1.45-1.41(\mathrm{~m}, 4 \mathrm{H}), 1.33-1.29(\mathrm{~m}, 12 \mathrm{H}), 0.92-0.89(\mathrm{~m}, 6 \mathrm{H}), 0.17(\mathrm{~s}$, $12 \mathrm{H}) .{ }^{13} \mathrm{C} \mathrm{NMR}\left(100 \mathrm{MHz}, \mathrm{CDCl}_{3}, 298 \mathrm{~K}\right): \delta$ (ppm) 152.5, 124.3, 35.8, 32.0, 29.5, 29.2, 22.9, 14.3, 0.7. HRMS (FI): calcd for $\mathrm{C}_{20} \mathrm{H}_{42} \mathrm{OSi}_{2}[\mathrm{M}]^{+}: 354.2769$; Found: 354.2766 .<smiles>C=C(c1ccccc1)[Si](C)(C)O[Si](C)(C)c1ccccc1</smiles>
1,1,3,3-Tetramethyl-1,3-bis(1-phenylvinyl)disiloxane (P55) The reaction was carried out according to general procedure by using phenylacetylene (204 mg, $2.0 \mathrm{mmol}$ ), 
$\left(\mathrm{HSiMe}_{2}\right)_{2} \mathrm{O}(134 \mathrm{mg}, 1 \mathrm{mmol})$ and catalyst $1(106 \mathrm{mg}, 0.1 \mathrm{mmol})$ at $60{ }^{\circ} \mathrm{C}$ for $5 \mathrm{~h}$. Purification by fast silica gel column chromatography using petroleum ether as the eluent afforded the title compound as a colorless oil (243 $\mathrm{mg}$, 72\%). ${ }^{1} \mathrm{H}$ NMR (400 MHz, $\left.\mathrm{CDCl}_{3}, 294 \mathrm{~K}\right) \delta(\mathrm{ppm}) 7.33-7.20(\mathrm{~m}, 10 \mathrm{H}), 5.87(\mathrm{~d}, 2 \mathrm{H}, J=2.9 \mathrm{~Hz}), 5.65(\mathrm{~d}, 2 \mathrm{H}, J=$ $2.9 \mathrm{~Hz}), 0.20$ (s, 12H). ${ }^{13} \mathrm{C} \mathrm{NMR}\left(100 \mathrm{MHz}, \mathrm{CDCl}_{3}, 298 \mathrm{~K}\right): \delta$ (ppm) 152.6, 143.8, 128.3, 127.7, 127.0, 126.6, 1.0. HRMS (EI): calcd for $\mathrm{C}_{20} \mathrm{H}_{26} \mathrm{OSi}_{2}[\mathrm{M}]^{+}$: 338.1517; Found: 338.1526 .<smiles>C=C(c1ccccc1)[Si](C)(C)O[Si](C)(C)O[Si](C)(C)C(=C)c1ccccc1</smiles>

1-Phenylvinyl functionalized polydimethylsiloxane (P56) The reaction was carried out according to general procedure by using phenylacetylene $(204 \mathrm{mg}, 2.0 \mathrm{mmol})$, Hydride terminated polydimethylsiloxane $(1.3 \mathrm{~g}, 1.0 \mathrm{mmol})$ and catalyst $1(106 \mathrm{mg}$, $0.1 \mathrm{mmol})$ at $60{ }^{\circ} \mathrm{C}$ for $5 \mathrm{~h}$. The solvent was removed by vacuum to give the crude product, which was dissolved in hexane $(15 \mathrm{ml})$ and washed with water $(3 \times 5 \mathrm{ml})$, dried with $\mathrm{Na}_{2} \mathrm{SO}_{4}$, filtered and evaporated to dryness to give the product as a clear, slightly reddish brown oil $(0.8 \mathrm{~g}, 53 \%) .{ }^{1} \mathrm{H} \mathrm{NMR} \mathrm{(400} \mathrm{MHz,}$ $\left.\mathrm{CDCl}_{3}, 294 \mathrm{~K}\right) \delta(\mathrm{ppm}) 7.27-7.26(\mathrm{~m}, 3 \mathrm{H}), 7.22-7.17(\mathrm{~m}, 2 \mathrm{H}), 5.87(\mathrm{~d}, 1 \mathrm{H}, J=2.8 \mathrm{~Hz}), 5.67(\mathrm{~d}, 1 \mathrm{H}, J=2.8 \mathrm{~Hz})$, 0.23 (s, 6H), 0.06 (s, 48H). ${ }^{13} \mathrm{C}$ NMR (100 MHz, $\left.\mathrm{CDCl}_{3}, 294 \mathrm{~K}\right): \delta$ (ppm) 152.4, 143.8, 128.2, 127.6, 127.0, 126.6, 1.2, 1.0. Average molecular weight determined by NMR: $1010(\mathrm{~m}=16)$.

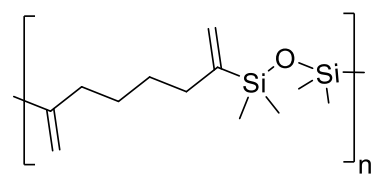

Poly(1,1,3,3-tetramethyl-1-(octa-1,7-dien-2-yl)disiloxane) (P57) The reaction was carried out according to general procedure by using octa-1,7-diyne (106 mg, $1.0 \mathrm{mmol})$, 1,1,3,3-tetramethyldisiloxane $(134 \mathrm{mg}, 1.0 \mathrm{mmol})$ and catalyst $1(0.05 \mathrm{~mol}, 53 \mathrm{mg})$ at $60{ }^{\circ} \mathrm{C}$ for $5 \mathrm{~h}$. The mixture was added dropwise to a mixture solvent $n$-hexane $(5 \mathrm{ml})$ and ethyl alcohol $(5 \mathrm{ml})$, then standing at $-15{ }^{\circ} \mathrm{C}$ for overnight. Pouring out the solvent slowly and dring under vacuum to leave $\mathbf{P 5 7}$ as a sticky light reddish brown oil (153.6 mg, 64\%). ${ }^{1} \mathrm{H}$ NMR (400 MHz, $\mathrm{CDCl}_{3}, 298 \mathrm{~K}$ ): $\delta$ (ppm) 5.57-5.38 (br, 4H), 2.15 (br, 4H), 1.40 (br, 4H), 0.15 (br, 12H). $\left.{ }^{13} \mathrm{C} \mathrm{NMR} \mathrm{(400} \mathrm{MHz,} \mathrm{CDCl}_{3}, 298 \mathrm{~K}\right): \delta(\mathrm{ppm})$ $152.2,124.4,35.5,29.1,0.7 . \mathrm{M}_{\mathrm{n}}=12647, \mathrm{M}_{\mathrm{w}} / \mathrm{M}_{\mathrm{n}}=1.6$.

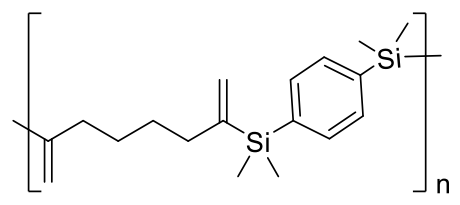

Poly((4-(dimethylsilyl)phenyl)dimethyl(octa-1,7-dien-2-yl)silane) (P58) The reaction was carried out according to general procedure by using octa-1,7-diyne $(106 \mathrm{mg}, 1.0 \mathrm{mmol}), 1,4-b i s($ dimethylsilyl)benzene $(194 \mathrm{mg}, 1.0 \mathrm{mmol})$ and catalyst $1(0.05 \mathrm{~mol}, 53 \mathrm{mg})$ at $60{ }^{\circ} \mathrm{C}$ for $5 \mathrm{~h}$. Then the mixture was added dropwise to a mixture solvent $n$-hexane $(5 \mathrm{ml})$ and ethyl alcohol $(5 \mathrm{ml})$, then standing at $-15{ }^{\circ} \mathrm{C}$ for overnight. Pouring out the solvent slowly and dring under vacuum to leave $\mathbf{P 5 8}$ as a sticky light reddish brown oil (204 mg, 68\%). ${ }^{1} \mathrm{H}$ NMR (400 MHz, $\mathrm{CDCl}_{3}, 303 \mathrm{~K}$ ): $\delta$ (ppm) 7.48 (br, 4H), 5.62 (br, 4H), 2.08 (br, 4H), 1.32 (br, 4H), 0.32 (br, 12H). ${ }^{13} \mathrm{C}$ NMR (400 MHz, $\mathrm{CDCl}_{3}, 303 \mathrm{~K}$ ): $\delta$ (ppm) 150.5, 139.3, 133.4, 126.0, 36.0, 28.7, -2.7. $\mathrm{M}_{\mathrm{n}}=16605$, $\mathrm{M}_{\mathrm{w}} / \mathrm{M}_{\mathrm{n}}=1.7$.

\section{Gram-Scale Reactions.}

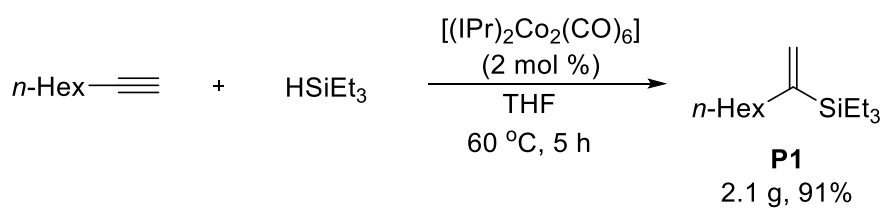

In a $\mathrm{N}_{2}$ glovebox, to a $100 \mathrm{~mL}$ screw-cap vial equipped with a stir bar was added oct-1-yne (10 mmol, $1.1 \mathrm{~g}), \mathrm{HSiEt}_{3}(11 \mathrm{mmol}, 1.2 \mathrm{~g})$, catalyst $1(0.2 \mathrm{mmol}, 213 \mathrm{mg})$ and THF $(20 \mathrm{~mL})$. The reaction mixture was stirred at $60{ }^{\circ} \mathrm{C}$ for $5 \mathrm{~h}$. After cooling to room temperature, the mixture was then exposed to air and was added $n$-hexane $(2 \mathrm{~mL})$. The organic phase was subjected to vacuum to 
remove the volatiles. The residue was then purified by fast silica gel column chromatography using petroleum ether as the eluent afforded the title compound as a colorless oil $(2.1 \mathrm{~g}, 91 \%)$.

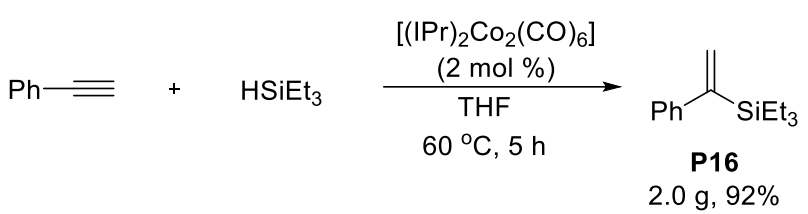

In a $\mathrm{N}_{2}$ glovebox, to a $100 \mathrm{~mL}$ screw-cap scintillation vial equipped with a stir bar was added Phenylacetylene (10 mmol, $1.0 \mathrm{~g})$, Triethyl silane (11 mmol, $1.2 \mathrm{~g})$, catalyst $1(0.2 \mathrm{mmol}, 212.6 \mathrm{mg})$ and THF $(20 \mathrm{~mL})$. The reaction mixture was stirred at $60{ }^{\circ} \mathrm{C}$ for $5 \mathrm{~h}$. After cooling to room temperature, the mixture was then exposed to air and was added $n$-hexane $(2 \mathrm{~mL})$. The organic phase was subjected to vacuum to remove the volatiles. The residue was then purified by fast silica gel column chromatography using petroleum ether as the eluent afforded the title compound as a colorless oil $(2.0 \mathrm{~g}, 92 \%)$.

\section{Palladium-Catalyzed Hiyama Coupling.}

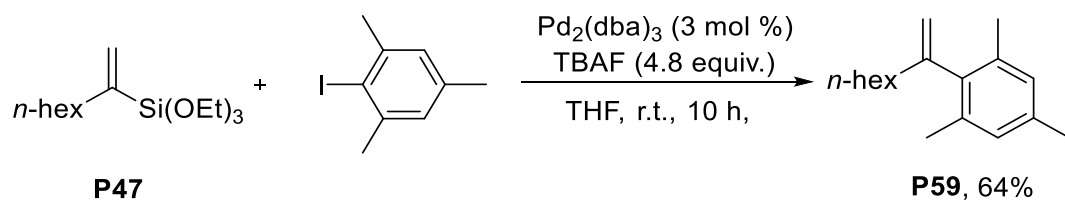

In an $\mathrm{N}_{2}$-filled glovebox, a Schlenk flask (10 mL) was charged with P47 (411 $\left.\mathrm{mg}, 1.5 \mathrm{mmol}\right)$ and TBAF (tetra- $n$-butylammonium fluoride, $4.8 \mathrm{~mL}, 4.8 \mathrm{mmol}, 1.0 \mathrm{M}$ in THF). The resulted mixture was stirred for $3 \mathrm{~min}$. at room temperature. $\operatorname{Pd}_{2}(\mathrm{dba})_{3}(27 \mathrm{mg}, 0.03 \mathrm{mmol})$ and 2-iodo-1,3,5-trimethylbenzene (246 mg, $1.0 \mathrm{mmol}$ ) was then introduced and the reaction mixture was stirred at room temperature for $10 \mathrm{~h}$. The mixture was concentrated under vacuum. The residue was then purified by column chromatography using petroleum ether as eluent to give the desired product (147 mg, 64\%). ${ }^{1} \mathrm{H}$ NMR (400 MHz, $\left.\mathrm{CDCl}_{3}, 293 \mathrm{~K}\right): \delta(\mathrm{ppm}) 6.85$ (s, 2H), $5.23(\mathrm{~m}, 1 \mathrm{H}), 4.79(\mathrm{~d}, 1 \mathrm{H}$, $J=1.5 \mathrm{~Hz}), 2.27(\mathrm{~s}, 3 \mathrm{H}), 2.20(\mathrm{~s}, 6 \mathrm{H}), 2.17(\mathrm{t}, 2 \mathrm{H}, J=9.2 \mathrm{~Hz}), 1.53-1.44(\mathrm{~m}, 2 \mathrm{H}), 137-1.26(\mathrm{~m}, 6 \mathrm{H})$, $0.88(\mathrm{t}, 3 \mathrm{H}, J=6.7 \mathrm{H}) .{ }^{13} \mathrm{C} \mathrm{NMR}\left(100 \mathrm{MHz}, \mathrm{CDCl}_{3}, 293 \mathrm{~K}\right): 149.1,140.6,135.8,135.0,128.2,113.0$, $37.3,32.0,29.5,27.6,22.8,21.1,20.0,14.3$. These data are in accordance with the literature. ${ }^{25}$

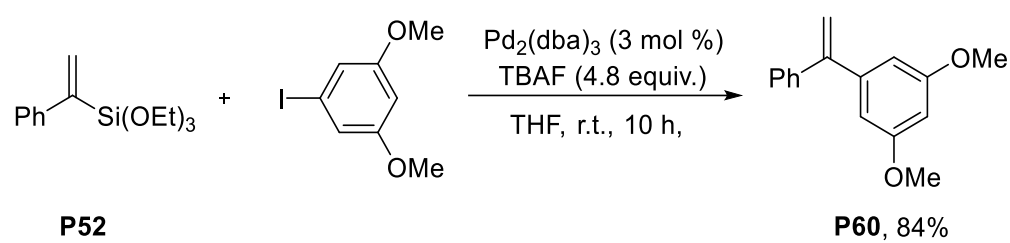

In an $\mathrm{N}_{2}$-filled glovebox, a Schlenk flask (10 mL) was charged with $\mathbf{P 5 2}$ (399.2 $\mathrm{mg}, 1.5 \mathrm{mmol}$ ) and TBAF (tetra- $n$-butylammonium fluoride, $4.8 \mathrm{~mL}, 4.8 \mathrm{mmol}, 1.0 \mathrm{M}$ in THF). The resulted mixture was stirred for $3 \mathrm{~min}$. at room temperature. $\mathrm{Pd}_{2}(\mathrm{dba})_{3}(27.5 \mathrm{mg}, 0.03 \mathrm{mmol})$ and 1-iodo-3,5-dimethoxybenzene $(264.0 \mathrm{mg}, 1 \mathrm{mmol})$ was then introduced and the reaction mixture was stirred at room temperature for $10 \mathrm{~h}$. The mixture was concentrated under vacuum. The residue was then purified by column chromatography using PE/EA (20:1) as eluent to give the desired product (202 mg, 84\%). ${ }^{\cdot 1} \mathrm{H}$ NMR (400 MHz, $\left.\mathrm{CDCl}_{3}, 294 \mathrm{~K}\right): \delta(\mathrm{ppm}) 7.37-7.31(\mathrm{~m}, 5 \mathrm{H}), 6.50(\mathrm{~d}, 2 \mathrm{H}, J=$ 
$2.2 \mathrm{~Hz}), 6.45$ (t, 1H, J = 2.2 Hz), $5.46(\mathrm{~s}, 2 \mathrm{H}), 3.77(\mathrm{~s}, 6 \mathrm{H}) .{ }^{13} \mathrm{C} \mathrm{NMR}\left(100 \mathrm{MHz}, \mathrm{CDCl}_{3}, 294 \mathrm{~K}\right): \delta$ (ppm) 160.6, 150.1, 143.8, 141.2, 128.3, 128.2, 127.8, 114.5, 106.7, 99.9, 55.5. These data are in accordance with the literature. ${ }^{26}$ 
6. Kinetic Profiles of the Reaction of Phenyl Acetylene with HSiEt3 Using 1-5 as Catalysts. The reaction was carried out according to the general procedures A by using phenyl acetylene $(0.50$ mmol), $\mathrm{HSiEt}_{3}(0.55 \mathrm{mmol})$, catalysts 1 (5 mol\%), 2 (5 mol\%), 3 (5 mol\%), 4 (10 mol\%), 5 (5 mol\%), and $n$-dodecane $(0.50 \mathrm{mmol})$ in THF $(1 \mathrm{~mL})$. The reaction mixture was stirred at $60{ }^{\circ} \mathrm{C}$ or room temperature and monitored by GC at regular intervals (10 mins or 1 hour). The change of the yield of $\alpha$-triethylsilyl styrene according time is shown in Figure $4 \mathrm{c}$.

\section{Deuterium-Labeling Experiments}

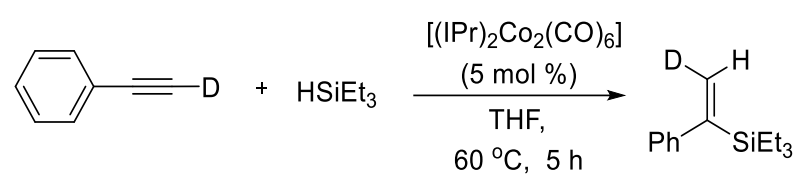

In a $\mathrm{N}_{2}$ glovebox, to a $10 \mathrm{~mL}$ screw-cap vial equipped with a stir bar was added PhCCD $(0.5 \mathrm{mmol}$, $51 \mathrm{mg}), \mathrm{HSiEt}_{3}(0.5 \mathrm{mmol}, 58 \mathrm{mg})$, catalyst $1(0.025 \mathrm{mmol}, 26 \mathrm{mg})$ and THF $(1 \mathrm{~mL})$. The reaction mixture was stirred at $60{ }^{\circ} \mathrm{C}$ for $5 \mathrm{~h}$. The mixture was then exposed to air and $n$-hexane $(1 \mathrm{~mL})$ was added. The organic layer was separated and purified by fast silica gel column chromatography using petroleum ether as the eluent afforded the title mixture compound as a colorless oil $(97 \mathrm{mg}, 89 \%) .{ }^{1} \mathrm{H}$ NMR (400 MHz, $\left.\mathrm{CDCl}_{3}, 303 \mathrm{~K}\right): \delta(\mathrm{ppm})$ 7.37-7.33 (m, 2H), 7.28-7.27 (m, 1H), 7.23-7.21 (m, 2H), $5.63(\mathrm{~s}, 1 \mathrm{H}), 1.00(\mathrm{t}, 9 \mathrm{H}, J=7.9 \mathrm{~Hz}), 0.74(\mathrm{q}, 6 \mathrm{H}, J=7.9 \mathrm{~Hz}) .{ }^{2} \mathrm{H} \mathrm{NMR}\left(61 \mathrm{MHz}, \mathrm{CDCl}_{3}, 296 \mathrm{~K}\right): \delta$ (ppm) 5.16. ${ }^{13} \mathrm{C} \mathrm{NMR}\left(100 \mathrm{MHz}, \mathrm{CDCl}_{3}, 303 \mathrm{~K}\right): \delta(\mathrm{ppm}) 150.4,145.6,128.7(\mathrm{t}, J=23.7 \mathrm{~Hz}), 128.2$, 126.8, 126.2, 7.5, 3.4. HRMS (EI): calcd for $\mathrm{C}_{14} \mathrm{H}_{21} \mathrm{DSi}[\mathrm{M}]^{+}: 219.1548$; Found: 219.154 .

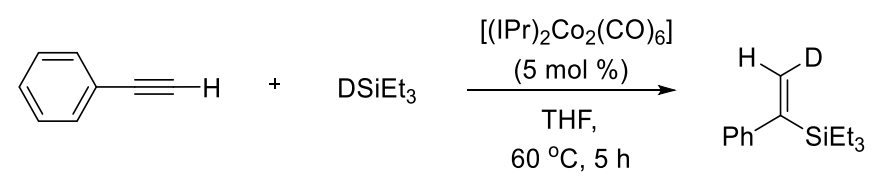

In a $\mathrm{N}_{2}$ glovebox, to a $10 \mathrm{~mL}$ screw-cap vial equipped with a stir bar was added $\mathrm{PhCCH}(0.50$ mmol, $51 \mathrm{mg})$, DSiEt $3(0.50 \mathrm{mmol}, 58 \mathrm{mg})$, catalyst $1(0.025 \mathrm{~mol}, 26 \mathrm{mg})$ and THF $(1 \mathrm{~mL})$. The reaction mixture was stirred at $60{ }^{\circ} \mathrm{C}$ for $5 \mathrm{~h}$. The mixture was then exposed to air and $n$-hexane ( 1 $\mathrm{mL}$ ) was added. The organic layer was separated and purified by fast silica gel column chromatography using petroleum ether as the eluent afforded the title mixture compound as a colorless oil (95 mg, 87\%). ${ }^{1} \mathrm{H}$ NMR (400 MHz, $\left.\mathrm{CDCl}_{3}, 298 \mathrm{~K}\right): \delta(\mathrm{ppm})$ 7.34-7.31 (m, 2H), 7.27-7.22 (m, 1H), 7.20-7.18 (m, 2H), $5.90(\mathrm{~s}, 1 \mathrm{H}), 0.97$ (t, 9H, $J=7.9 \mathrm{~Hz}), 0.71(\mathrm{q}, 6 \mathrm{H}, J=7.9 \mathrm{~Hz})$. ${ }^{2} \mathrm{H} \mathrm{NMR}\left(61 \mathrm{MHz}, \mathrm{CDCl}_{3}, 296 \mathrm{~K}\right): \delta(\mathrm{ppm}) 4.95 .{ }^{13} \mathrm{C} \mathrm{NMR}\left(100 \mathrm{MHz}, \mathrm{CDCl}_{3}, 303 \mathrm{~K}\right): \delta(\mathrm{ppm})$ $150.5,145.6,128.6(\mathrm{t}, J=24.2 \mathrm{~Hz}), 128.2$, 126.8, 126.2, 7.5, 3.5. HRMS (EI): calcd for $\mathrm{C}_{14} \mathrm{H}_{21} \mathrm{DSi}$ $[\mathrm{M}]^{+}:$219.1548; Found: 219.1553.

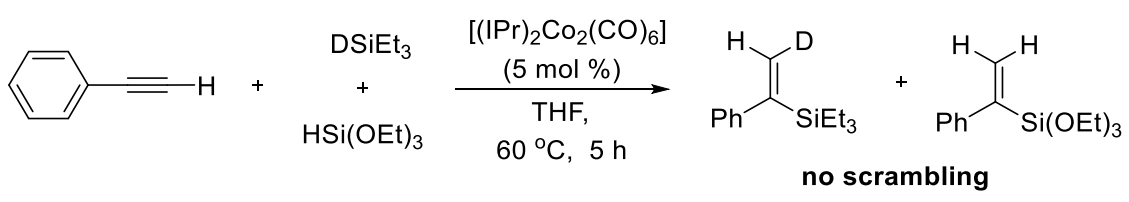

In a $\mathrm{N}_{2}$ glovebox, to a $10 \mathrm{~mL}$ screw-cap vial equipped with a stir bar was added $\mathrm{PhCCH}(0.5 \mathrm{mmol}$, $51 \mathrm{mg}), \mathrm{DSiEt}_{3}(0.3 \mathrm{mmol}, 35 \mathrm{mg}), \mathrm{HSi}(\mathrm{OEt})_{3}(0.3 \mathrm{mmol}, 35 \mathrm{mg})$, catalyst $1(0.025 \mathrm{mmol}, 26 \mathrm{mg})$ 
and THF $(1 \mathrm{~mL})$. The reaction mixture was stirred at $60{ }^{\circ} \mathrm{C}$ for $5 \mathrm{~h}$. The mixture was then exposed to air and $n$-hexane $(1 \mathrm{~mL})$ was added. The organic layer was separated and purified by fast silica gel column chromatography using petroleum ether as the eluent afforded the (Z)-triethyl(1-phenylvinyl-2-d)silane (52 mg, 80\%) and triethoxy(1-phenylvinyl)silane (61 mg, 76\%) as colorless oils. 


\section{Reference}

(1) Hans, M.; Lorkowski, J.; Demonceau, A.; Delaude, L. Efficient Synthetic Protocols for the Preparation of Common N-Heterocyclic Carbene Precursors. Beilstein J. Org. Chem. 2015, 11, 2318-2325.

(2) Levens, A.; An, F.; Breugst, M.; Mayr, H.; Lupton, D. W. Influence of the N-Substituents on the Nucleophilicity and Lewis Basicity of N-Heterocyclic Carbenes. Org. Lett. 2016, 18, 3566-3569.

(3) Gibson, S. E.; Johnstone, C.; Loch, J. A.; Steed, J. W.; Stevenazzi, A. Novel Structures and Pauson-Khand Activities of N-Heterocyclic Carbene Dicobalt Complexes. Organometallics 2003, 22, 5374-5377.

(4) Kamon, H.; Aoki, Y.; Nakata, N.; Ishii, A. Characterization and Phenylacetylene-Assisted Cyclometalation of an Isolable Hydrido-Selenolato $\mathrm{Pt}^{\mathrm{II}}$ Complex Having Phosphite Ligands, cis- $\left[\mathrm{PtH}(\mathrm{SeTrip})\left\{\mathrm{P}(\mathrm{OPh})_{3}\right\}_{2}\right]$. Bull. Chem. Soc. Jpn. 2014, 87, 274-282.

(5) He, T.; Wang, G.; Bonetti, V.; Klare, H. F. T.; Oestreich, M. Silylium-Ion-Promoted (5+1) Cycloaddition of Aryl-Substituted Vinylcyclopropanes and Hydrosilanes Involving Aryl Migration. Angew. Chem., Int. Ed. 2020, 59, 12186-12191.

(6) Sheldrick, G. M. SADABS, Program for Empirical Absorption Correction of Area Detector Data; University of Göttingen: Germany, 1996.

(7) Sheldrick, G. M. SHELXTL 5.10 for Windows NT: Structure Determination Software Programs; Bruker Analytical X-ray systems, Inc.: Madison, WI, 1997.

(8) Du, J.; Wang, L.; Xie, M.; Deng, L. A Two-Coordinate Cobalt(II) Imido Complex with NHC Ligation: Synthesis, Structure, and Reactivity. Angew. Chem. Int. Ed., 2015, 54, 12640-12644.

(9) Rivero-Crespo, M. A.; Leyva-Pérez, A.; Corma, A. A Ligand-Free $\mathrm{Pt}_{3}$ Cluster Catalyzes the Markovnikov Hydrosilylation of alkynes with up to $10^{6}$ Turnover Frequencies. Chem. Eur. J. 2017, 23, 1702-1708.

(10) Usanov, D. L.; Yamamoto, H. Formation of Five- and Seven-Membered Rings Enabled by the Triisopropylsilyl Auxiliary Group. Org. Lett. 2012, 14, 414-417.

(11) Junker, C. S.; Welker, M. E.; Day, C. S. Synthesis of 4-Aryl- and 4-Alkyl-2-silyl-1,3-butadienes and Their Diels-Alder/Cross-Coupling Reactions. J. Org. Chem. 2010, 75, 8155-8165.

(12) Guo, W.; Pleixats, R.; Shafir, A.; Parella, T. Rhodium Nanoflowers Stabilized by a Nitrogen-Rich PEG- Tagged Substrate as Recyclable Catalyst for the Stereoselective Hydrosilylation of Internal Alkynes. Adv. Synth. Catal. 2015, 357, 89-99.

(13) Gee, J. C.; Fuller, B. A.; Lockett, H.; Sedghi, G.; Robertson, C. M.; Luzyanin, K. V. Visible Light Accelerated Hydrosilylation of Alkynes using Platinum-[Acyclic Diaminocarbene] Photocatalysts. Chem. Commun. 2018, 54, 9450-9453.

(14)Battace, A.; Zair, T.; Doucet, H.; Santelli, M. Selective Synthesis of (E)-triethyl(2-arylethenyl)silane Derivatives by Reaction of Aryl Bromides with Triethyl Vinylsilane Catalyzed by a Palladium-Tetraphosphine Complex. J. Organomet. Chem. 2005, 690 3790-3802.

(15) Hamze, A.; Provot, O.; Brion, J.; Alami, M. Regiochemical Aspects of the Platinum Oxide Catalyzed Hydrosilylation of Alkynes. Synthesis 2007, 13, 2025-2036.

(16) Chauhan, B. P. S.; Sarka, A. Functionalized Vinylsilanes via Highly Efficient and Recyclable Pt-nanoparticle Catalysed Hydrosilylation of Alkynes. Dalton Trans., 2017, 46, 8709-8715.

(17) Lu, W.; Li, C.; Wu, X.; Xie, X.; Zhang Z. [Rh(COD)Cl $]_{2} / \mathrm{PPh}_{3}$-Catalyzed Dehydrogenative Silylation of Styrene Derivatives with NBE as a Hydrogen Acceptor. Organometallics 2020, 39, 3780-3788.

(18) Kita, Y.; Tobisu, N.; Chatani, N. Rhodium-Catalyzed Alkenylation of Nitriles via Silicon-Assisted C-CN Bond Cleavage. Org. Lett. 2010, 12, 1864-1867.

(19) Wang, P.; Yeo, X.; Loh, T. Copper-Catalyzed Highly Regioselective Silylcupration of Terminal Alkynes to Form $\alpha$-Vinylsilane. J. Am. Chem. Soc. 2011, 133, 1254-1256. 
(20) Chaulagain, M. R.; Mahandru, G. M.; Montgomery, J. Alkyne Hydrosilylation Catalyzed by Nickel Complexes of N-Heterocyclic Carbenes. Tetrahedron 2006, 62, 7560-7566.

(21) Takamitsu, S.; Motoharu, C.; Hiroaki, S.; Yuki, H.; Masaharu, A.; Keiji, Y. Cationic Palladium Complex-Catalyzed Hydrosilylative Cross-Coupling of Alkynes with Alkenes 1,4-Addition of Trichlorosilane to form 4-silyl-1-butene Framework. Bull. Chem. Soc. Jpn. 2007, 80, 1814-1823.

(22) Skrodzki, M.; Patroniak, V.; Pawluc, P. Schiff Base Cobalt(II) Complex-Catalyzed Highly Markovnikov-Selective Hydrosilylation of Alkynes. Org. Lett. 2021, 23, 663-667.

(23) Fotie, J.; Agbo, M. E.; Qu, F,; Tolar, T. Dichloro(ethylenediamine)platinum(II), a Water-Soluble Analog of the Antitumor Cisplatin, as a Heterogeneous Catalyst for a Stereoselective Hydrosilylation of Alkynes under Neat Condition. Tetrahedron Lett. 2020. 61, 152300.

(24) Chauhan, M.; Hauck, B. J.; Keller, L. P.; Boudjouk, P. Hydrosilylation of Alkynes Catalyzed by Platinum on Carbon. J. Organomet. Chem. 2002, 645, 1-13.

(25) Qin, L.; Ren, X.; Lu, Y.; Li, Y.; Zhou, J. Intermolecular Mizoroki-Heck Reaction of Aliphatic Olefins with High Selectivity for Substitution at the Internal Position. Angew. Chem. Int. Ed. 2012, 51, 5915-5919.

(26) Bess E. N.; Sigman, M. S. Distinctive Meta-Directing Group Effect for Iridium-Catalyzed 1,1-Diarylalkene Enantioselective Hydrogenation. Org. Lett. 2013, 15, 646-649.

(27) Jimenez, R.; Lopez, J. M.; Cervantes, J. Metal Supported Catalysts Obtained by Sol-gel in the Hydrosilylation of Phenylacetylene with $\mathrm{R}_{3} \mathrm{SiH}$ Organosilanes $\left(\mathrm{R}_{3}=\mathrm{Ph}_{3}, \mathrm{Ph}_{2} \mathrm{Me}\right.$, and $\left.\mathrm{PhMe}_{2}\right)$. Can. J. Chem. 2000, 78, 1491-1495. 
9. NMR Spectra of cobalt complexes 2-6 and hydrosilylation products.

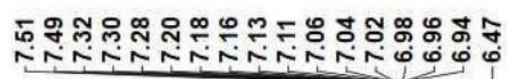

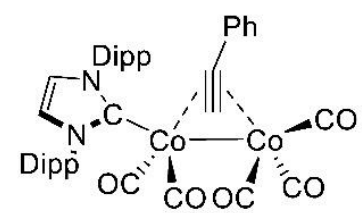

${ }^{1} \mathrm{H}$ NMR $\left(400 \mathrm{MHz}, \mathrm{C}_{6} \mathrm{D}_{6}, 298 \mathrm{~K}\right)$

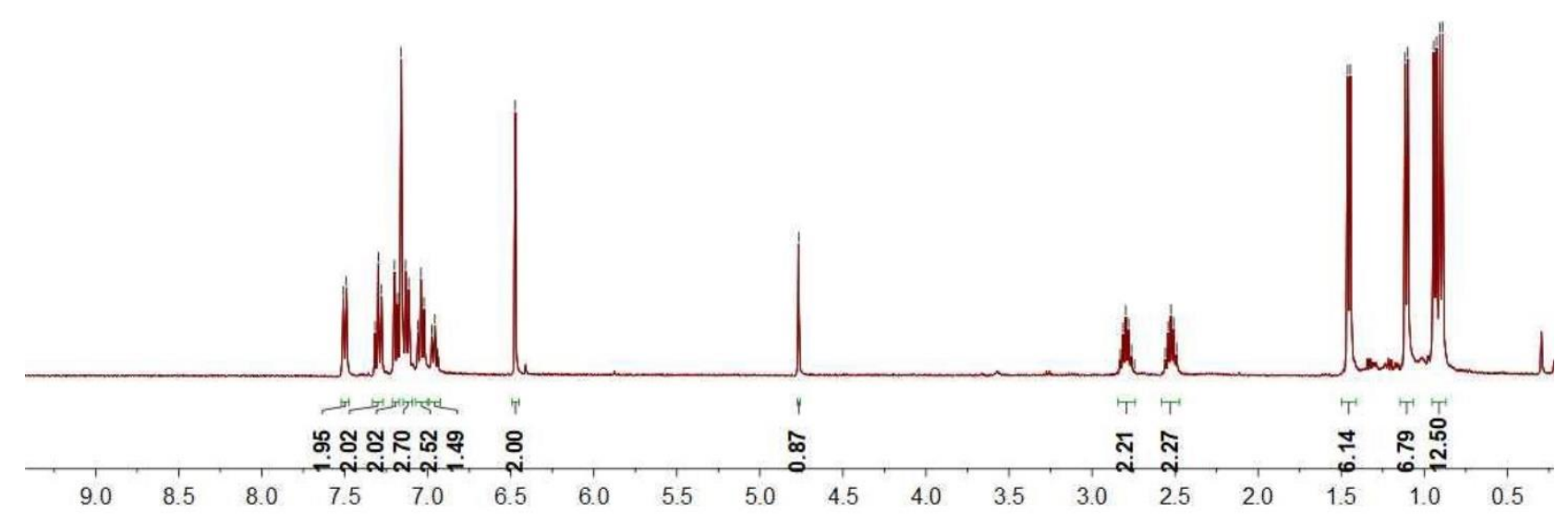

Figure S7. ${ }^{1} \mathrm{H}$ NMR spectrum of 2. 

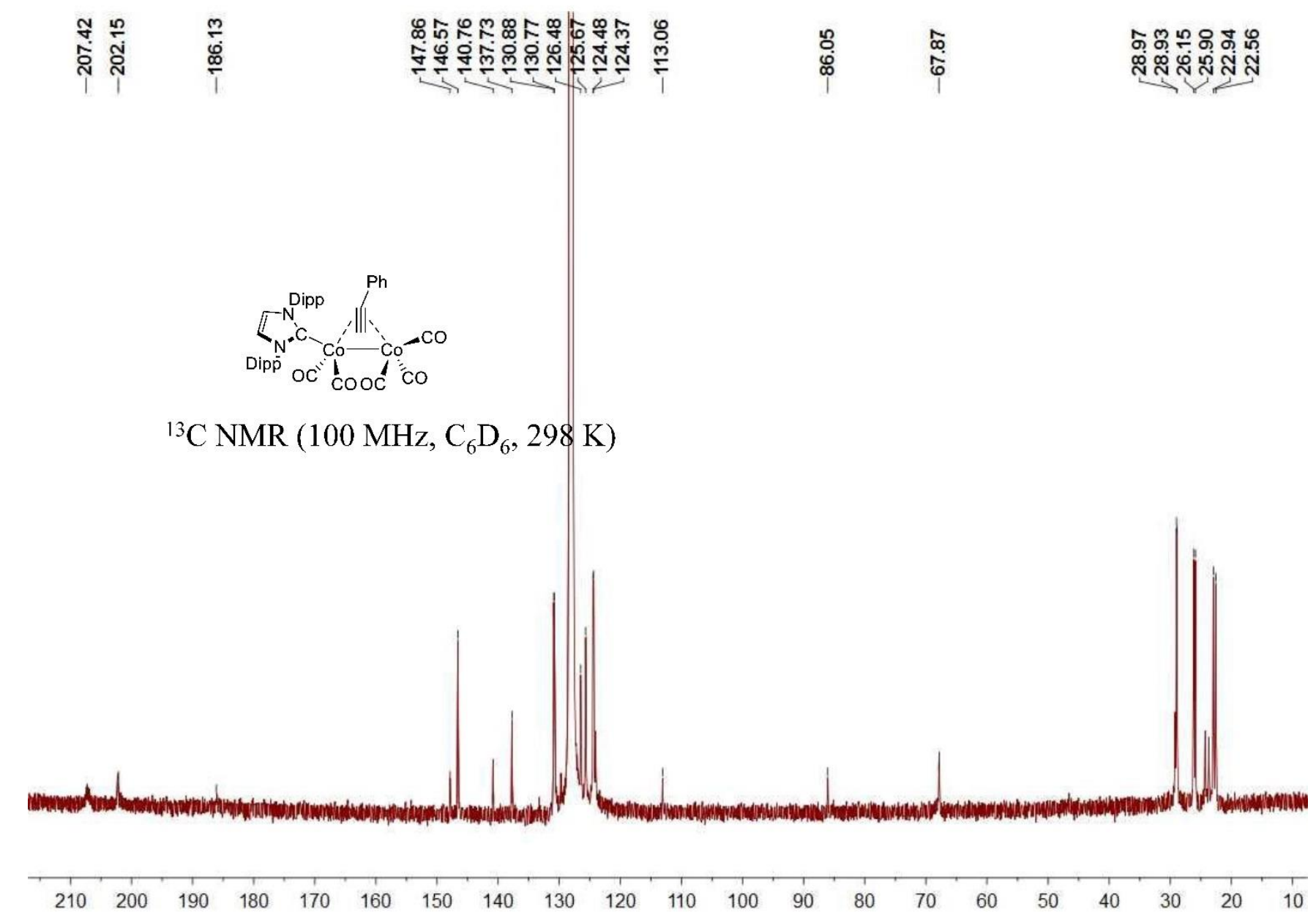

Figure S8. ${ }^{13} \mathrm{C}$ NMR spectrum of 2 . 


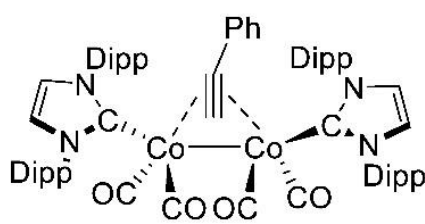

${ }^{1} \mathrm{H}$ NMR (400 MHz, $\mathrm{C}_{6} \mathrm{D}_{6}, 298 \mathrm{~K}$ )

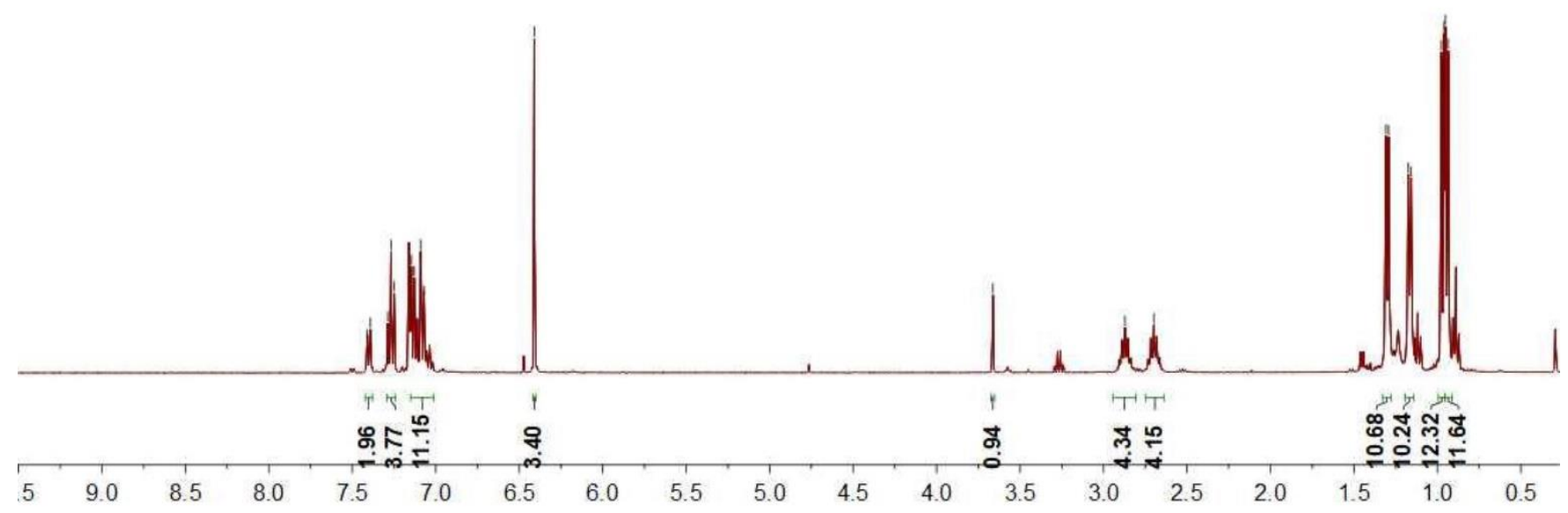

Figure S9. ${ }^{1} \mathrm{H}$ NMR spectrum of 3 . 


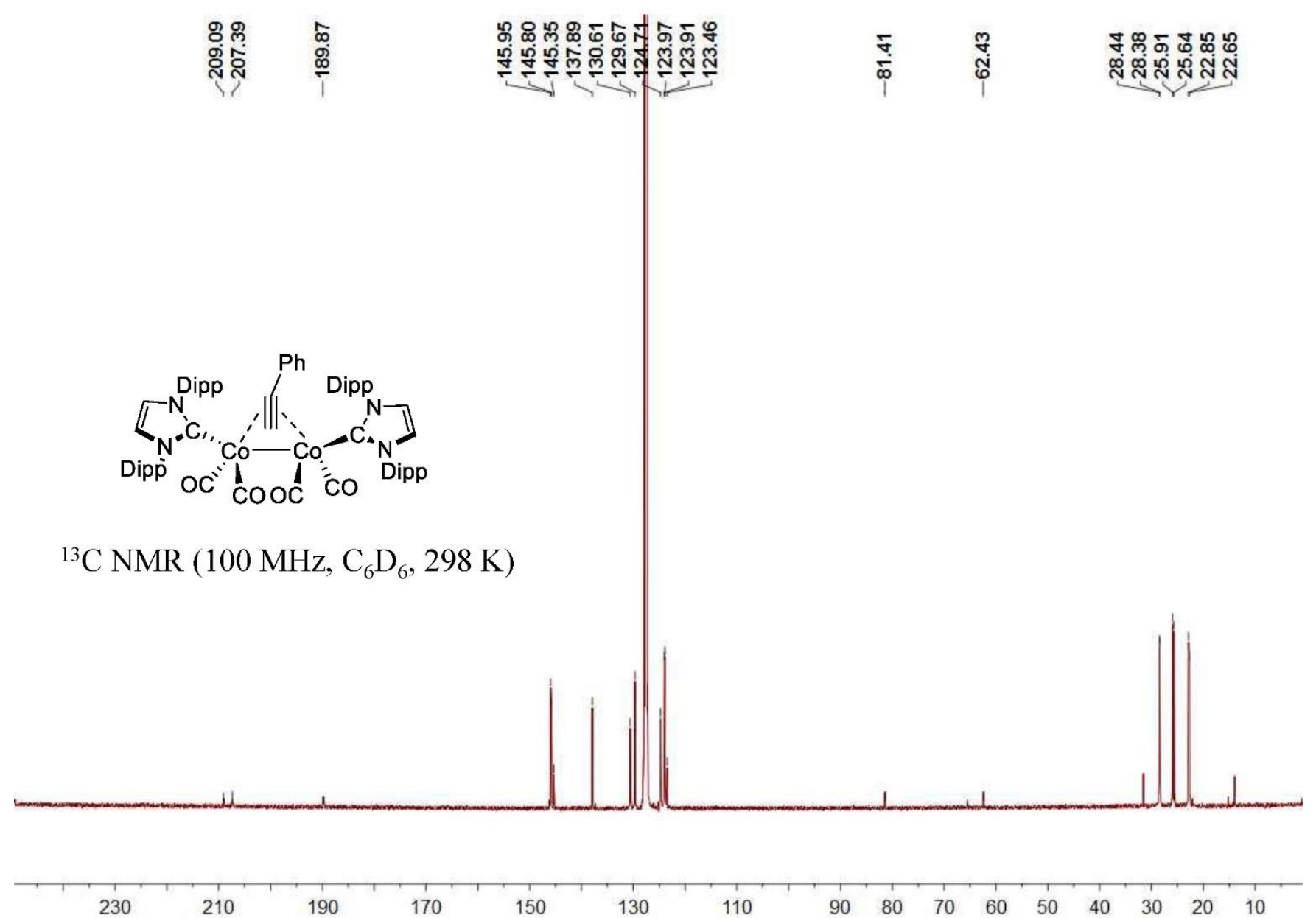

Figure S10. ${ }^{13} \mathrm{C}$ NMR spectrum of 3 . 


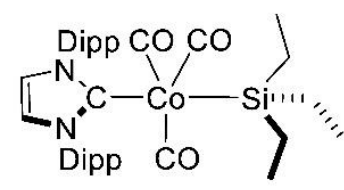

'H NMR (400 MHz, $\mathrm{C}_{6} \mathrm{D}_{6}, 295 \mathrm{~K}$ )

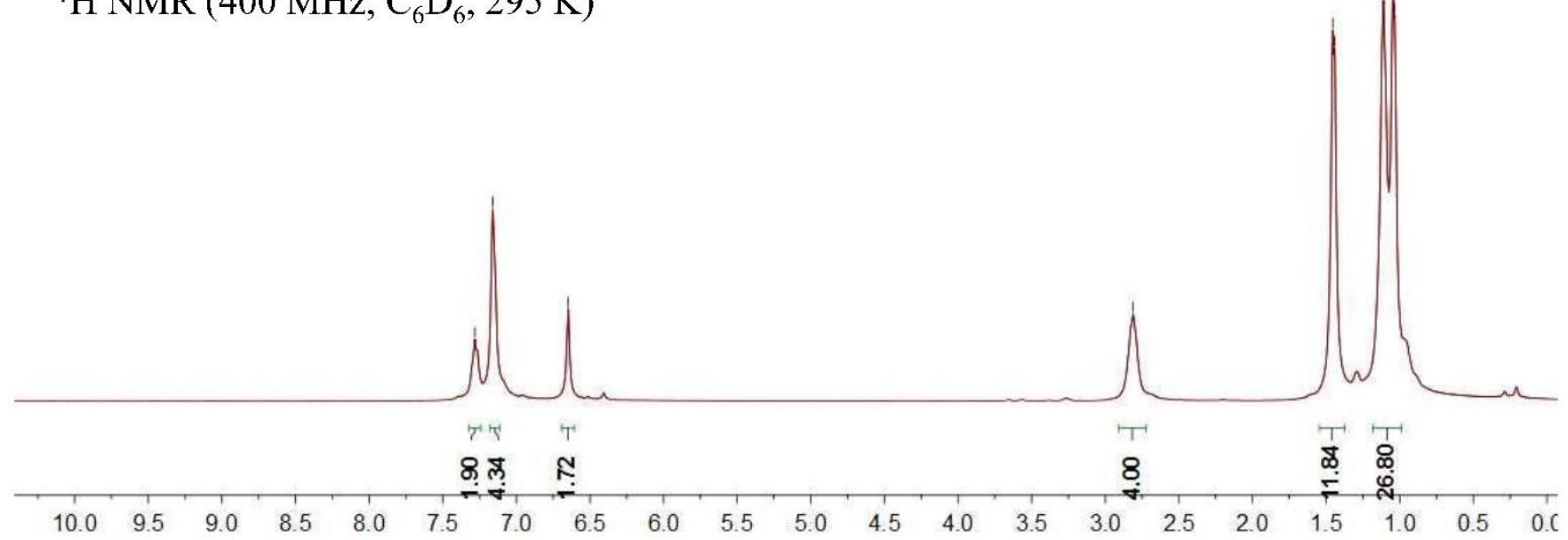

Figure S11. ${ }^{1} \mathrm{H}$ NMR spectrum of 4 . 


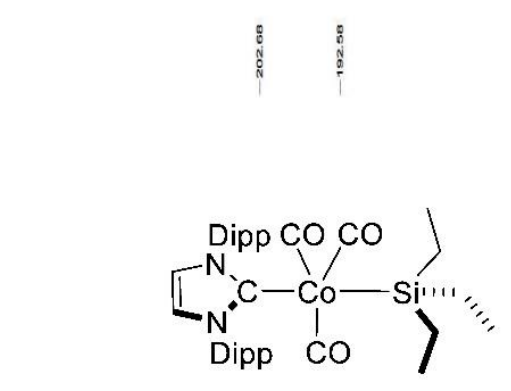

${ }^{13} \mathrm{C}$ NMR (100 MHz, $\left.\mathrm{C}_{6} \mathrm{D}_{6}, 295 \mathrm{~K}\right)$

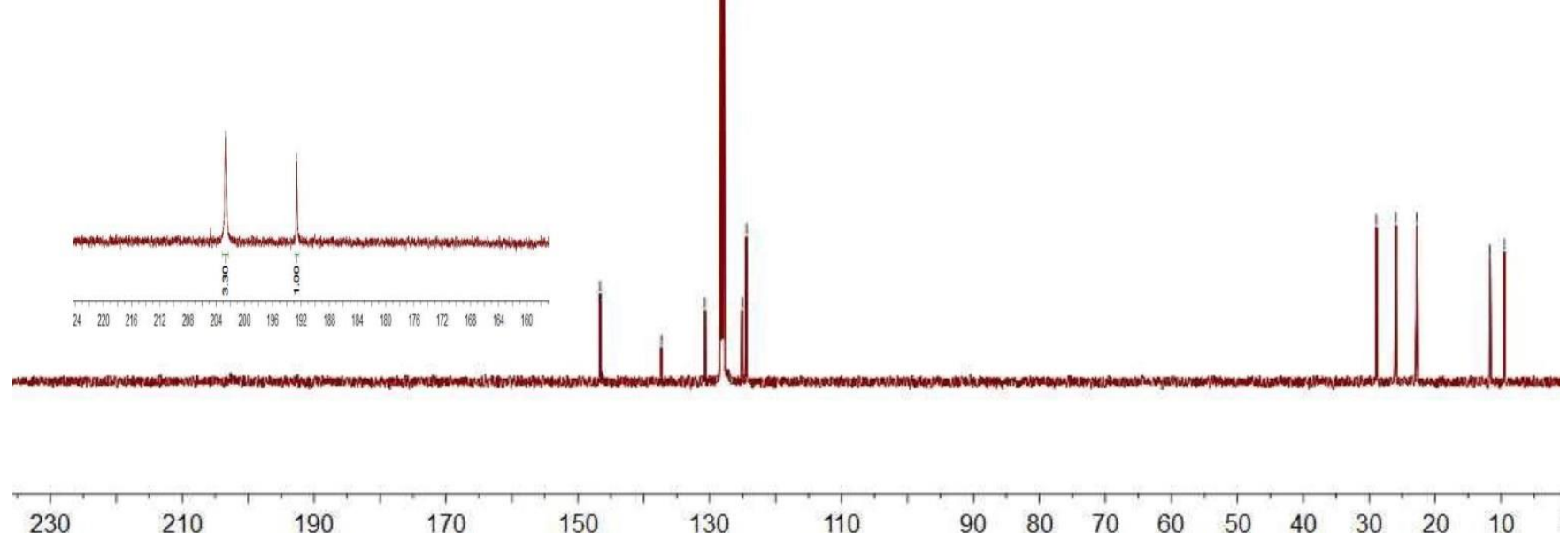

Figure S12. ${ }^{13} \mathrm{C}$ NMR spectrum of 4 . 


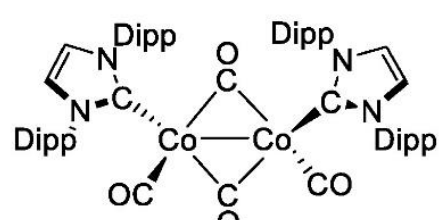

${ }^{1} \mathrm{H}$ NMR (400 MHz, $\mathrm{C}_{6} \mathrm{D}_{6}, 303 \mathrm{~K}$ )

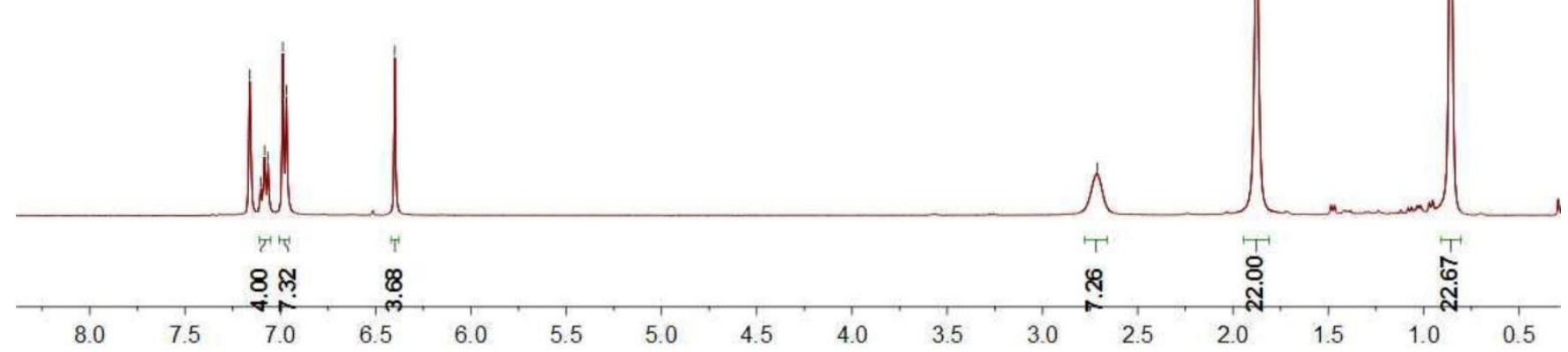

Figure S13. ${ }^{1} \mathrm{H}$ NMR spectrum of 5 . 


\section{0}

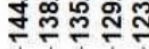

요요

สึ่ สู

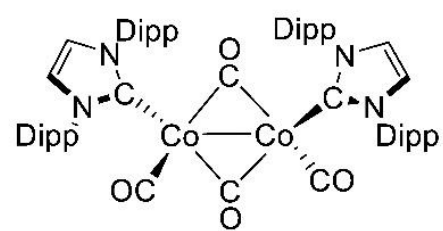

${ }^{13} \mathrm{C}$ NMR (100 MHz, $\left.\mathrm{C}_{6} \mathrm{D}_{6}, 303 \mathrm{~K}\right)$

$\begin{array}{lllllllllllllll}30 & 220 & 210 & 200 & 190 & 180 & 170 & 160 & 150 & 140 & 130 & 120 & 110 & 100 & 90\end{array}$

Figure S14. ${ }^{13} \mathrm{C}$ NMR spectrum of 5 . 


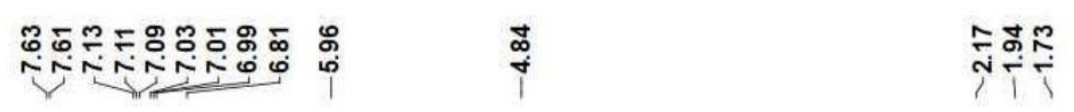

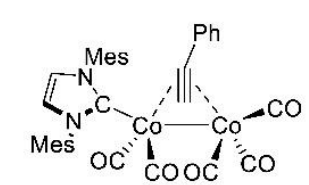

${ }^{1} \mathrm{H}$ NMR (400 MHz, $\mathrm{C}_{6} \mathrm{D}_{6}, 303 \mathrm{~K}$ )

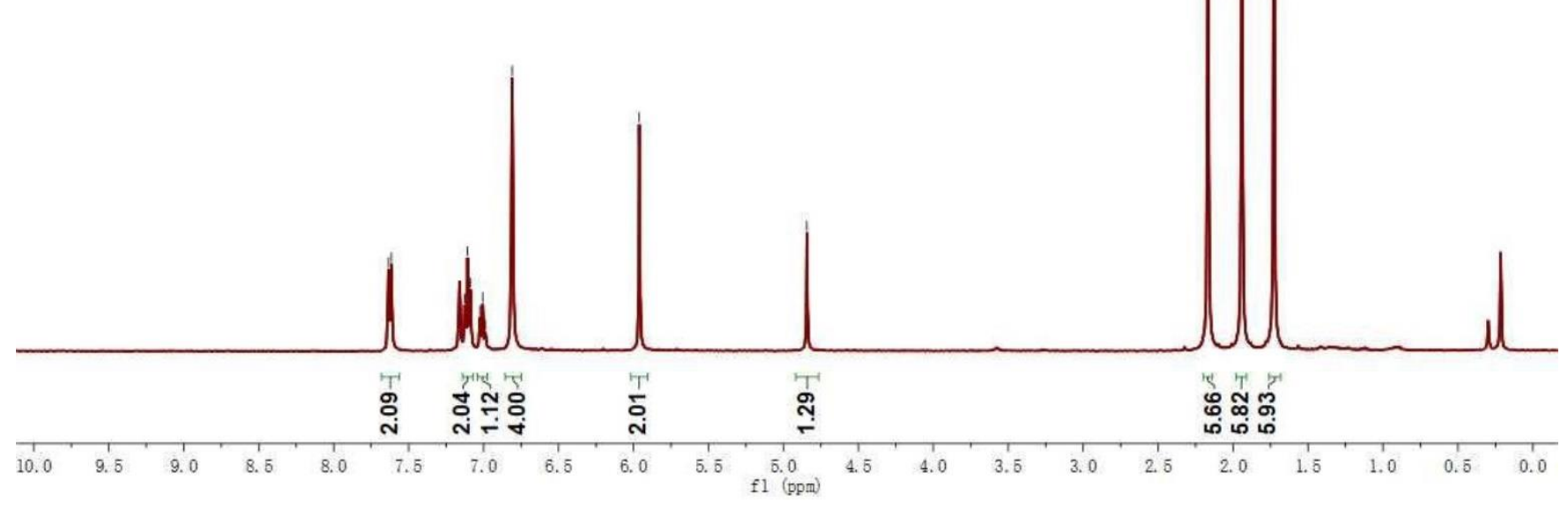

Figure S15. ${ }^{1} \mathrm{H}$ NMR spectrum of 6. 


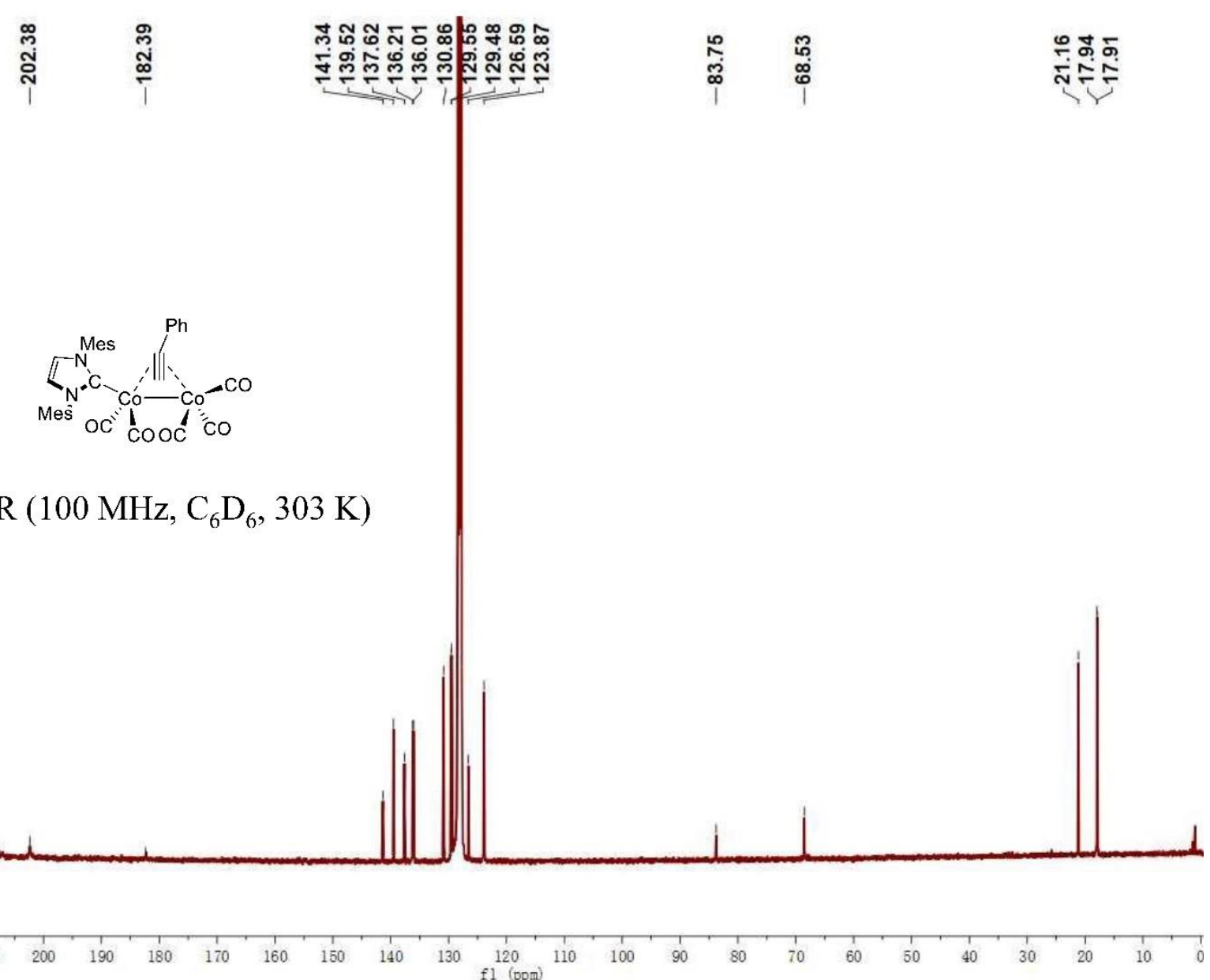

Figure S16. ${ }^{13} \mathrm{C}$ NMR spectrum of 6 . 


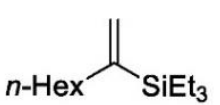

${ }^{1} \mathrm{H} \mathrm{NMR}\left(\mathrm{CDCl}_{3}, 400 \mathrm{MHz}, 298 \mathrm{~K}\right)$

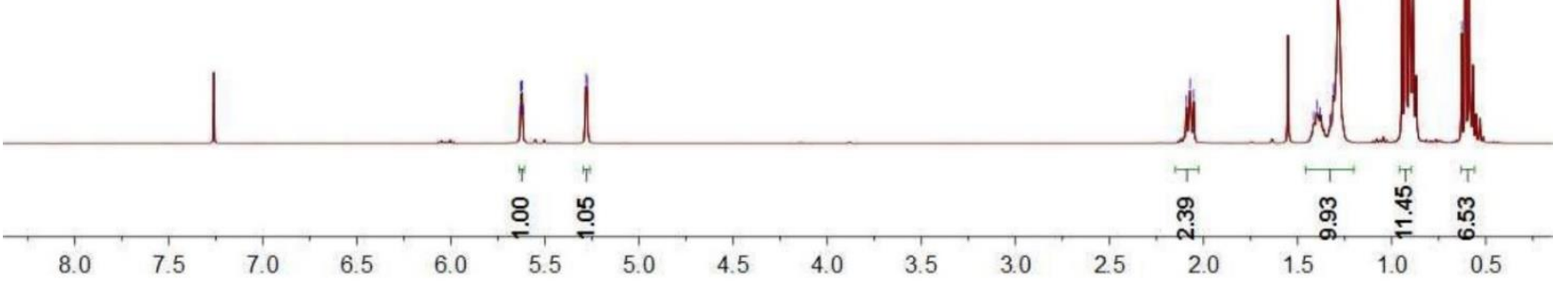

Figure S17. ${ }^{1} \mathrm{H}$ NMR spectrum of $\mathbf{P 1}$. 

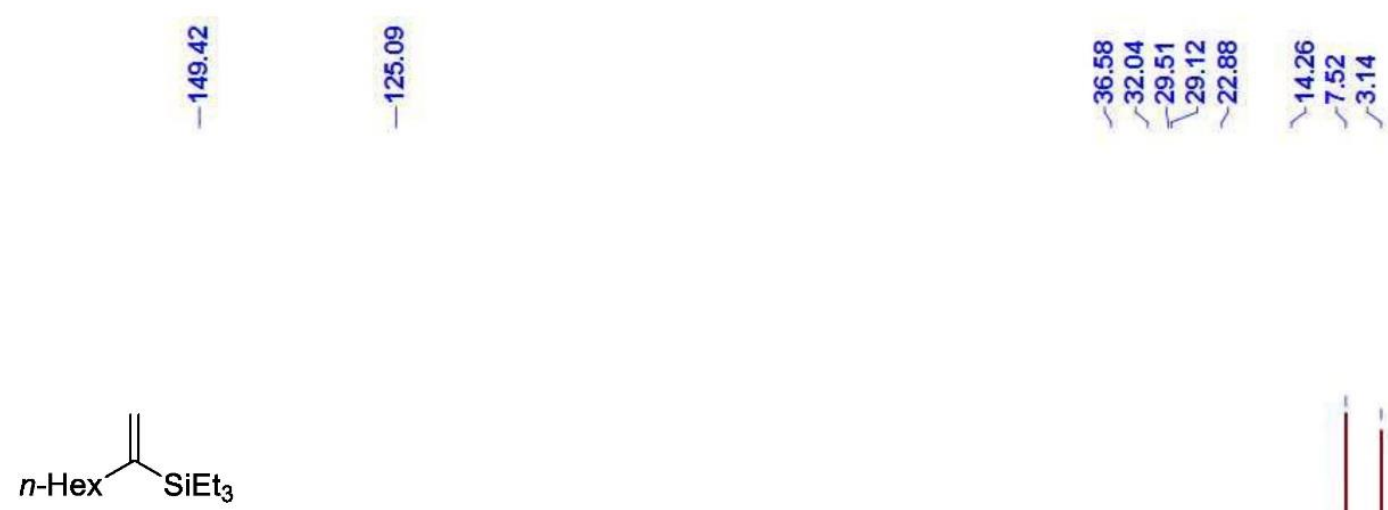

${ }^{13} \mathrm{C} \mathrm{NMR}\left(\mathrm{CDCl}_{3}, 100 \mathrm{MHz}, 298 \mathrm{~K}\right)$
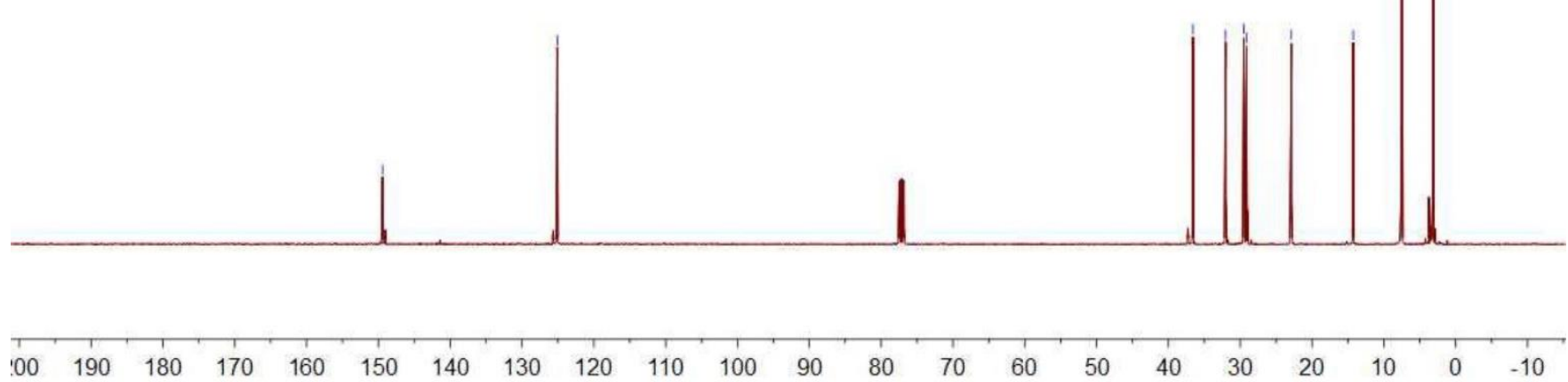

Figure S18. ${ }^{13} \mathrm{C}$ NMR spectrum of $\mathbf{P 1}$.

S45 


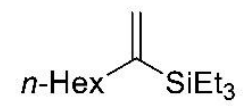

${ }^{29} \mathrm{Si} \mathrm{NMR}\left(79 \mathrm{MHz}, \mathrm{CDCl}_{3}, 297 \mathrm{~K}\right)$
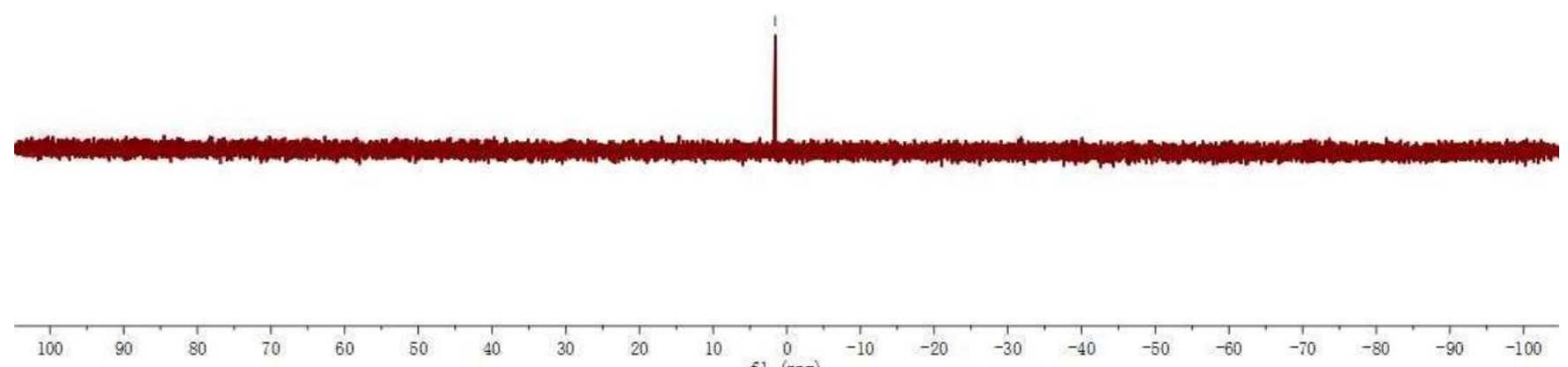

Figure S19. ${ }^{29}$ Si NMR spectrum of $\mathbf{P 1}$. 


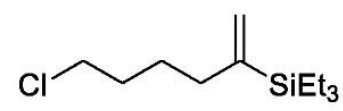

${ }^{1} \mathrm{H} \mathrm{NMR}\left(\mathrm{CDCl}_{3}, 400 \mathrm{MHz}, 298 \mathrm{~K}\right)$

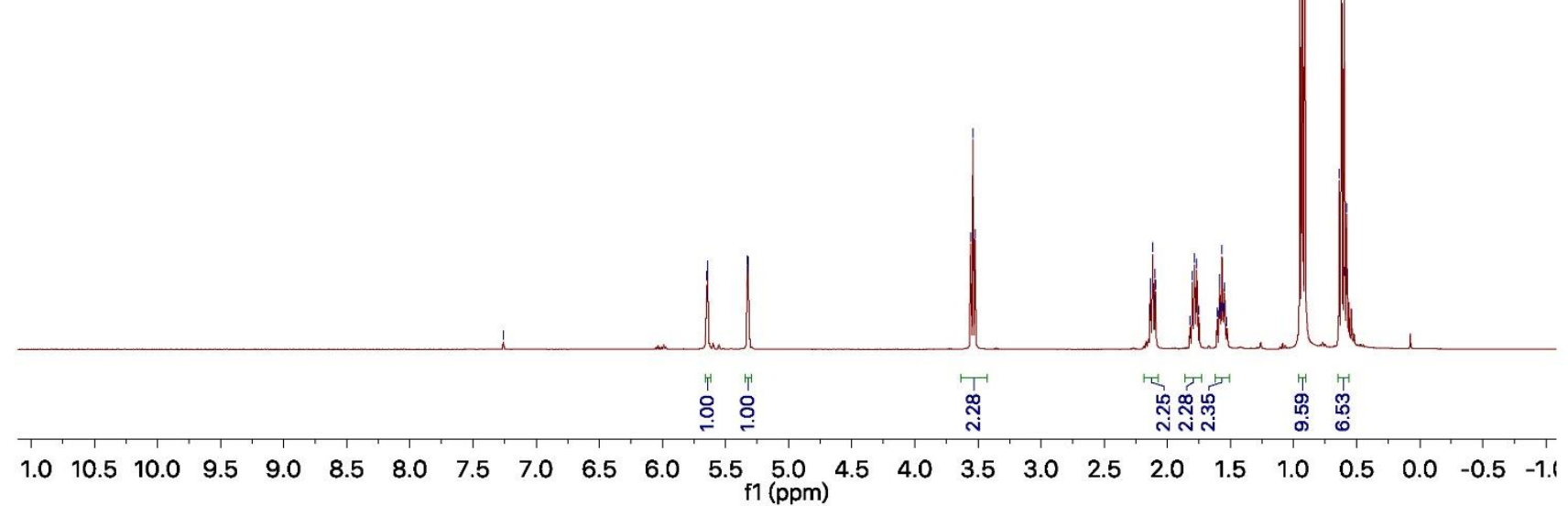

Figure S20. ${ }^{1} \mathrm{H}$ NMR spectrum of $\mathbf{P 2}$. 

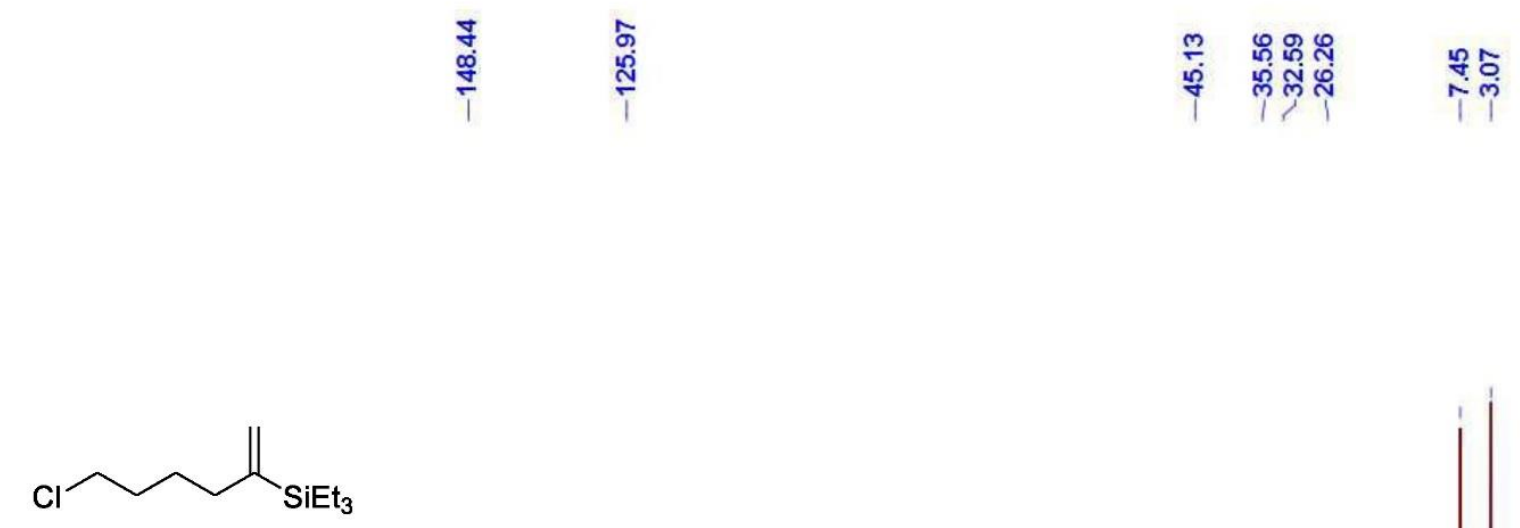

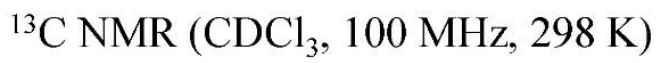

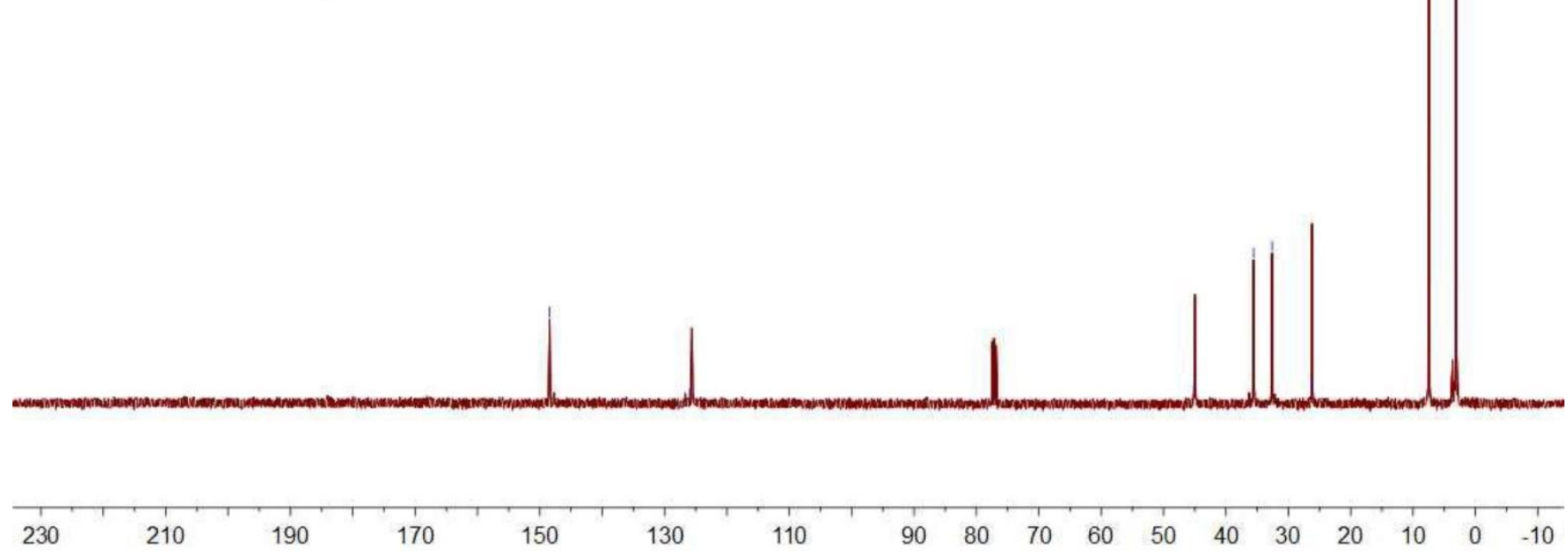

Figure S21. ${ }^{13} \mathrm{C}$ NMR spectrum of $\mathbf{P 2}$. 


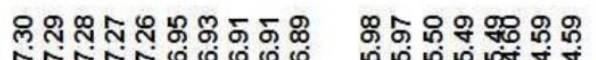

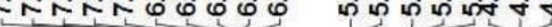

ஜ영중융융

10

$$
\mathrm{Ph}^{-\mathrm{O}} \mathrm{N}_{\mathrm{SiEt}_{3}}
$$

${ }^{1} \mathrm{H} \mathrm{NMR}\left(\mathrm{CDCl}_{3}, 400 \mathrm{MHz}, 293 \mathrm{~K}\right)$

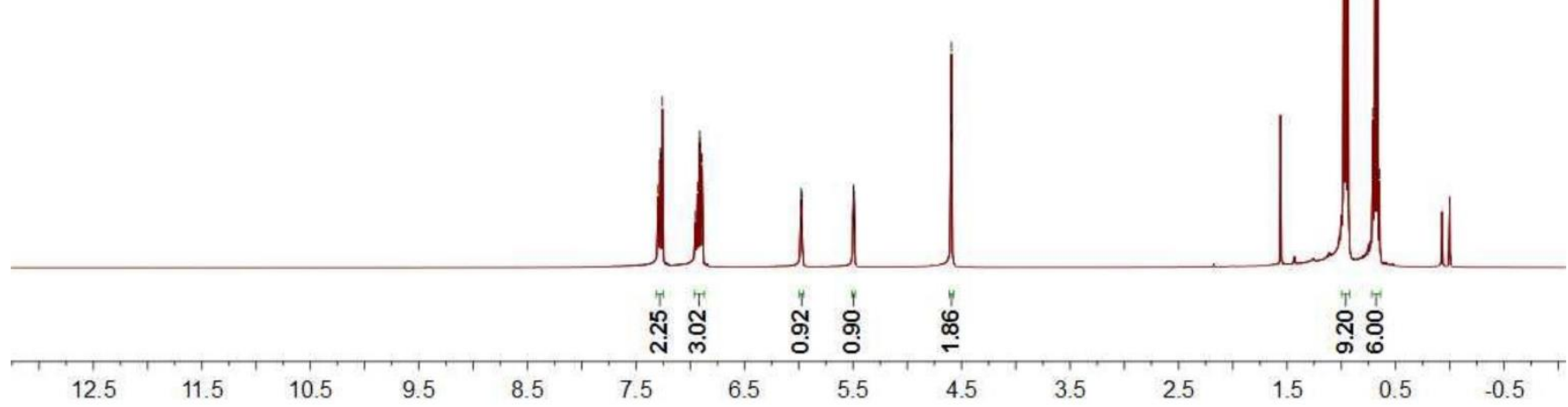

Figure S22. ${ }^{1} \mathrm{H}$ NMR spectrum of $\mathbf{P 3}$. 


$$
\mathrm{Ph}^{-}-\|_{\mathrm{SiEt}_{3}}
$$

${ }^{13} \mathrm{C} \mathrm{NMR}\left(\mathrm{CDCl}_{3}, 100 \mathrm{MHz}, 298 \mathrm{~K}\right)$

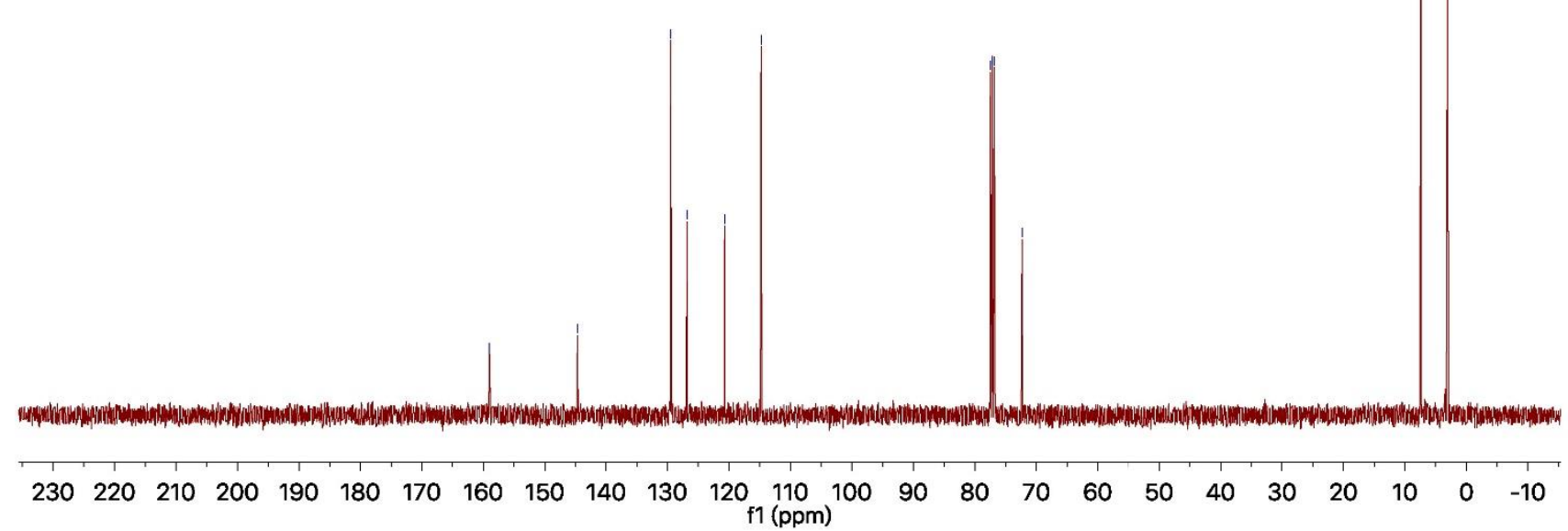

Figure S23. ${ }^{13} \mathrm{C}$ NMR spectrum of $\mathbf{P 3}$. 


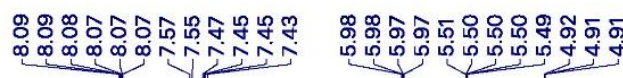

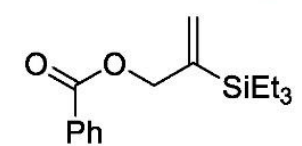

'H NMR (CDCl 3,400 MHz, 298 K)

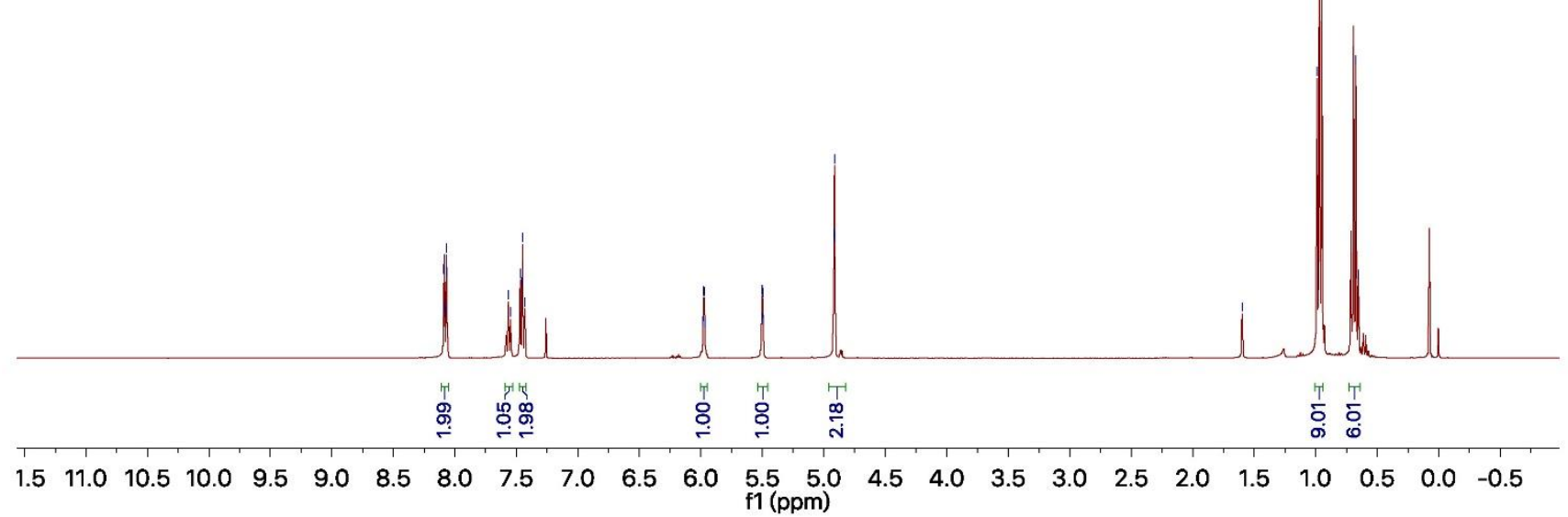

Figure S24. ${ }^{1} \mathrm{H}$ NMR spectrum of $\mathbf{P 4}$. 


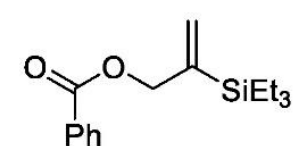

${ }^{13} \mathrm{C} \mathrm{NMR}\left(\mathrm{CDCl}_{3}, 100 \mathrm{MHz}, 298 \mathrm{~K}\right)$
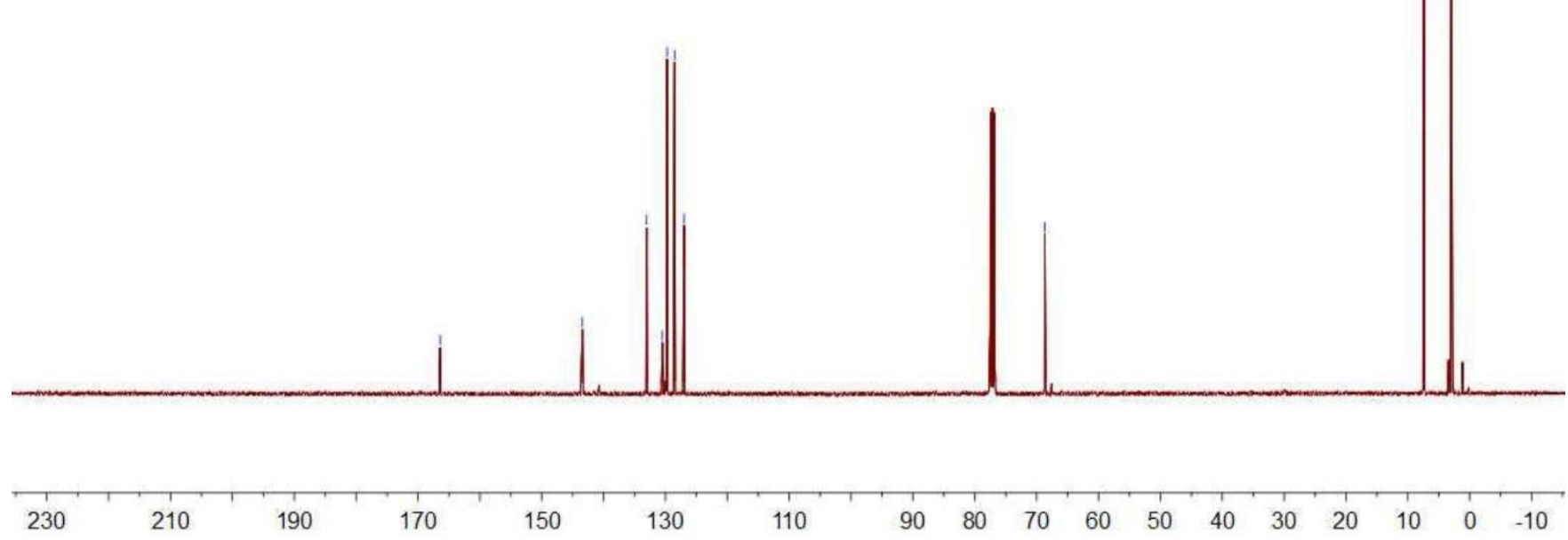

Figure S25. ${ }^{13} \mathrm{C}$ NMR spectrum of $\mathbf{P 4}$. 


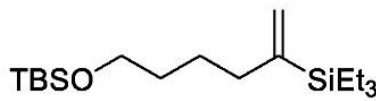

${ }^{1} \mathrm{H}$ NMR $\left(\mathrm{CDCl}_{3}, 400 \mathrm{MHz}, 296 \mathrm{~K}\right)$

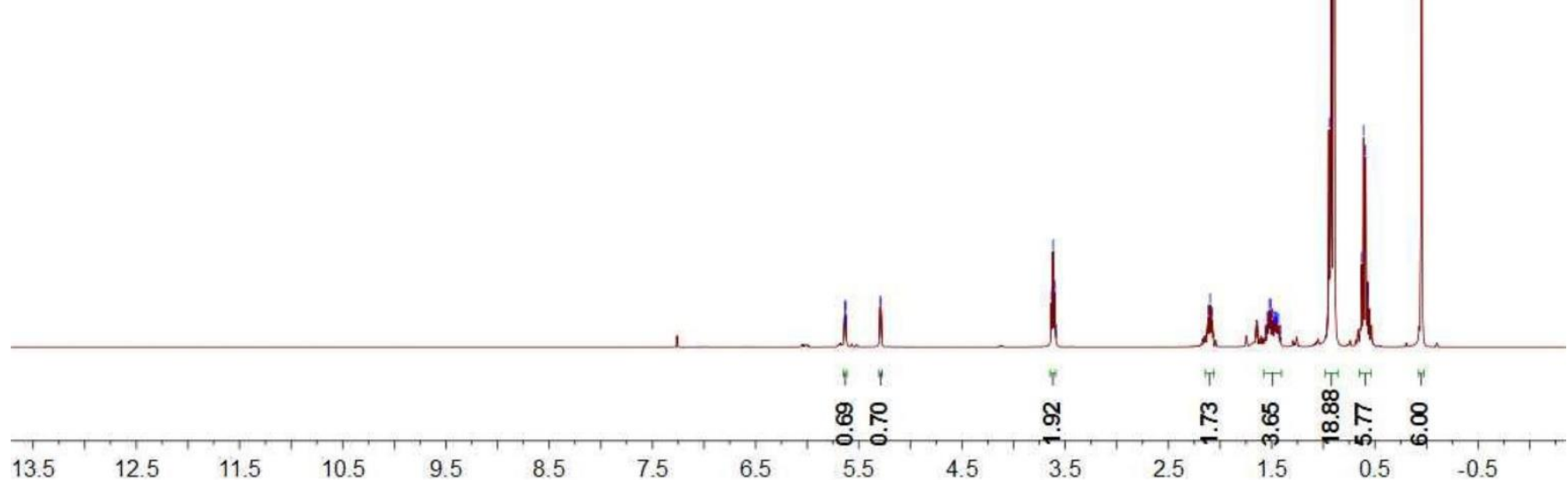

Figure S26. ${ }^{1} \mathrm{H}$ NMR spectrum of $\mathbf{P 5}$. 

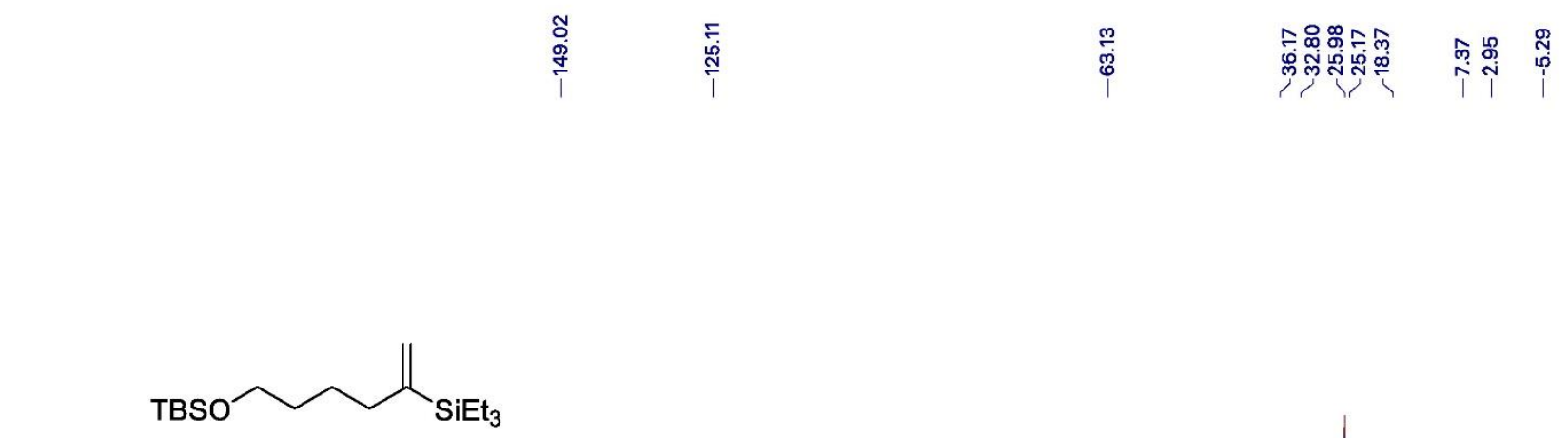

${ }^{13} \mathrm{C}$ NMR ( $\left.\mathrm{CDCl}_{3}, 100 \mathrm{MHz}, 296 \mathrm{~K}\right)$

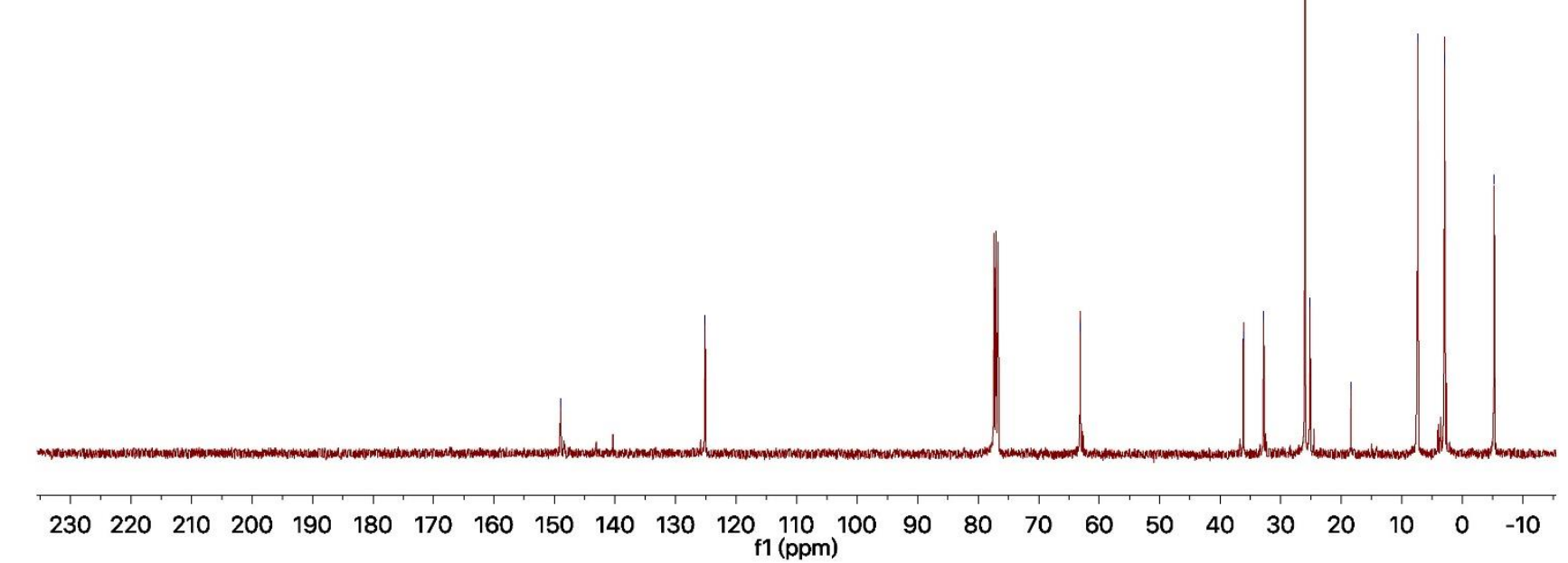

Figure S27. ${ }^{13} \mathrm{C}$ NMR spectrum of $\mathbf{P 5}$. 


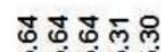

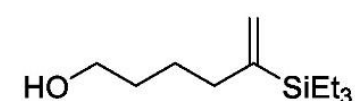

${ }^{1} \mathrm{H} \mathrm{NMR}\left(\mathrm{CDCl}_{3}, 400 \mathrm{MHz}, 298 \mathrm{~K}\right)$

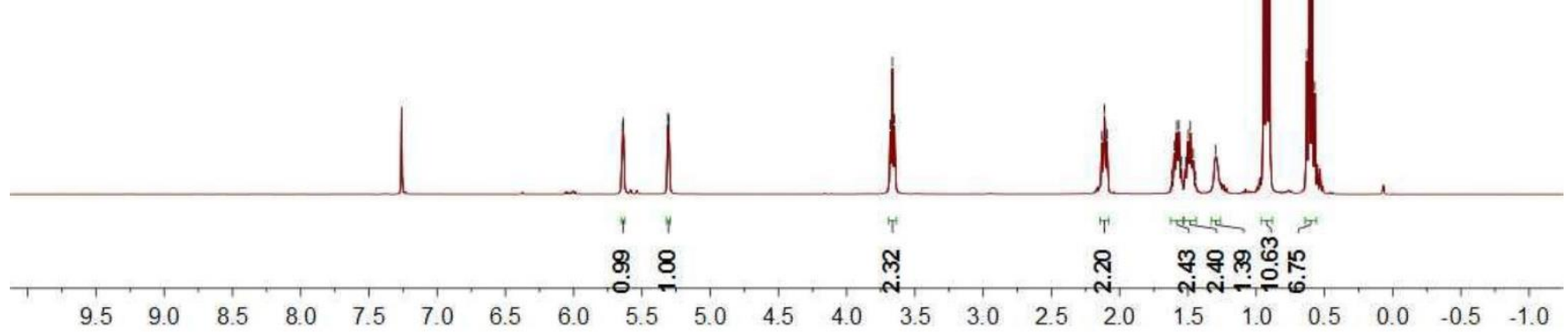

Figure S28. ${ }^{1} \mathrm{H}$ NMR spectrum of $\mathbf{P 6}$ 


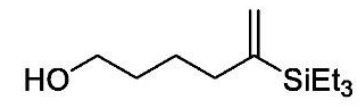

${ }^{13} \mathrm{C} \mathrm{NMR}\left(\mathrm{CDCl}_{3}, 100 \mathrm{MHz}, 295 \mathrm{~K}\right)$

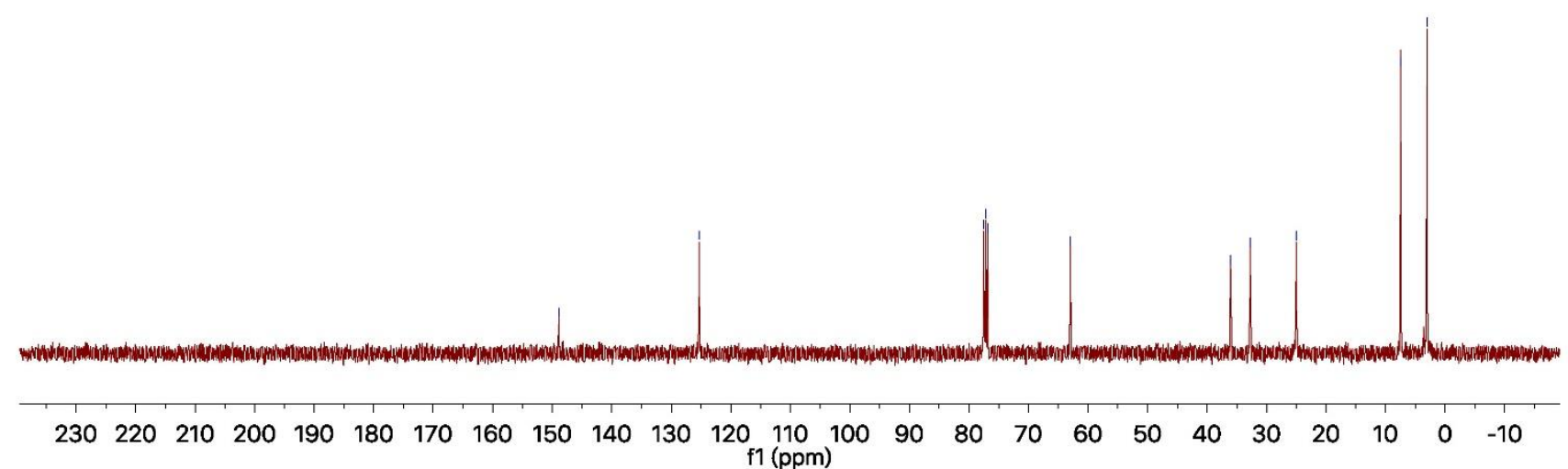

Figure S29. ${ }^{13} \mathrm{C}$ NMR spectrum of $\mathbf{P 6}$. 


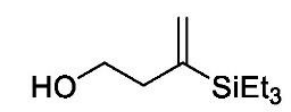

${ }^{1} \mathrm{H} \mathrm{NMR}\left(\mathrm{CDCl}_{3}, 400 \mathrm{MHz}, 293 \mathrm{~K}\right)$

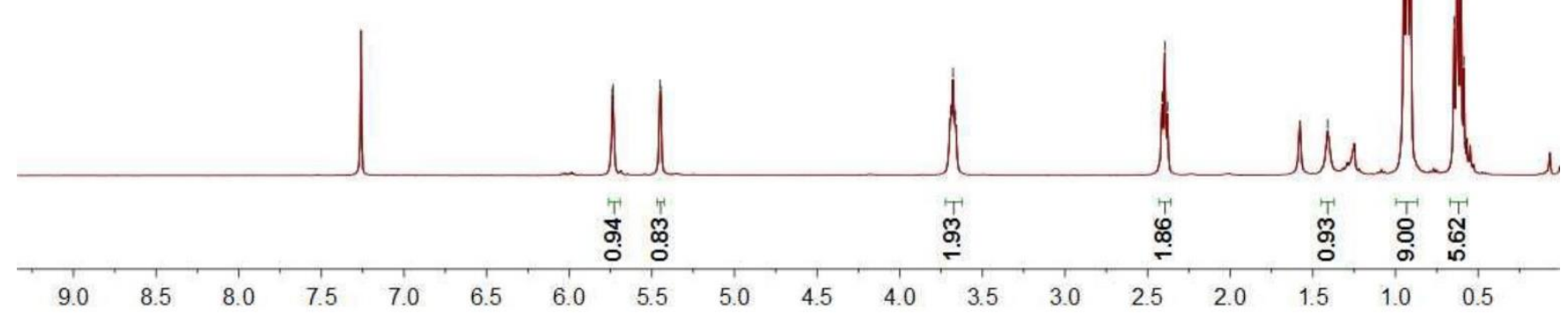

Figure S30. ${ }^{1} \mathrm{H}$ NMR spectrum of $\mathbf{P 7}$. 
$\mathrm{NO}_{\mathrm{SiEt}_{3}}$

${ }^{13} \mathrm{C} \mathrm{NMR}\left(\mathrm{CDCl}_{3}, 100 \mathrm{MHz}, 298 \mathrm{~K}\right)$

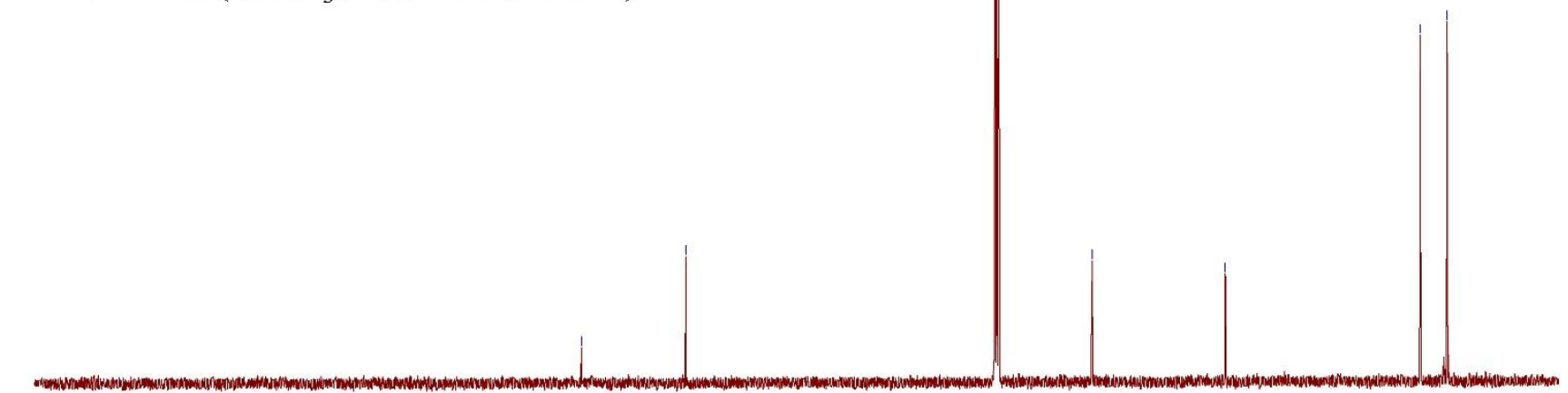

$\begin{array}{lllllllllllllllllllllllll}230 & 220 & 210 & 200 & 190 & 180 & 170 & 160 & 150 & 140 & 130 & 120 & 110 & 100 & 90 & 80 & 70 & 60 & 50 & 40 & 30 & 20 & 10 & 0 & -10\end{array}$

$$
\mathrm{f} 1(\mathrm{ppm})
$$

Figure S31. ${ }^{13} \mathrm{C}$ NMR spectrum of $\mathbf{P 7}$. 


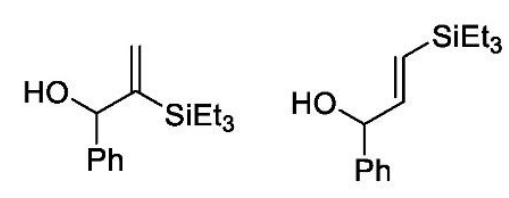

(3:1)

${ }^{1} \mathrm{H} \mathrm{NMR}\left(\mathrm{CDCl}_{3}, 400 \mathrm{MHz}, 298 \mathrm{~K}\right)$

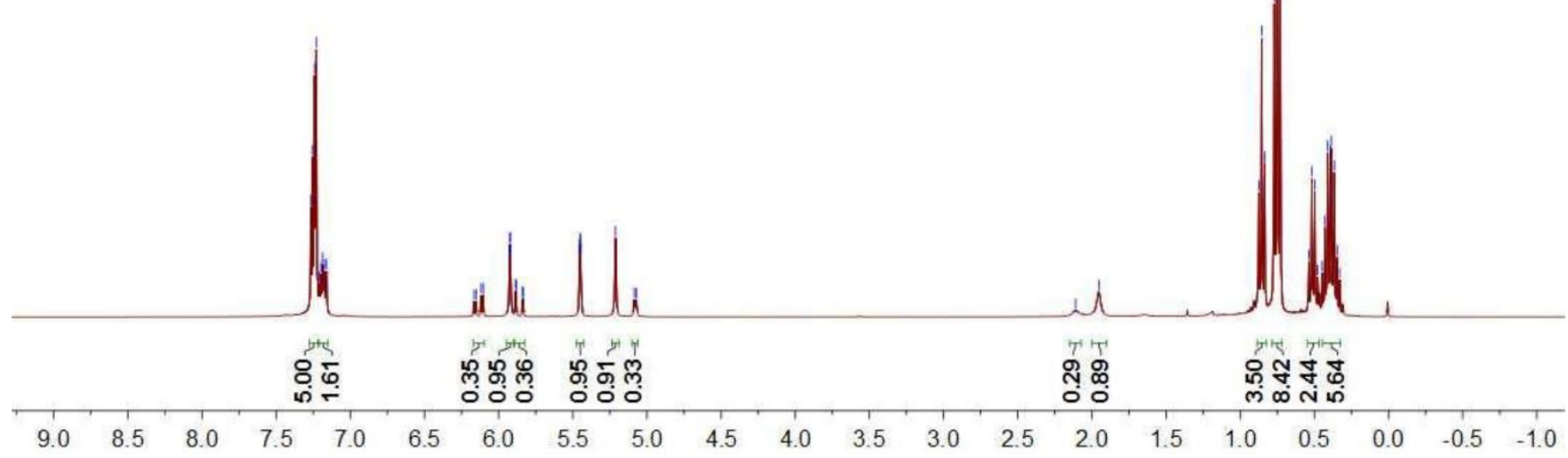

Figure S32. ${ }^{1} \mathrm{H}$ NMR spectrum of $\mathbf{P 8}$. 


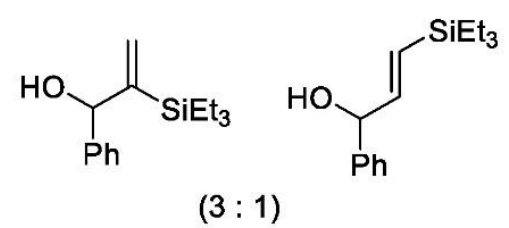

${ }^{13} \mathrm{C}$ NMR $\left(\mathrm{CDCl}_{3}, 100 \mathrm{MHz}, 295 \mathrm{~K}\right)$

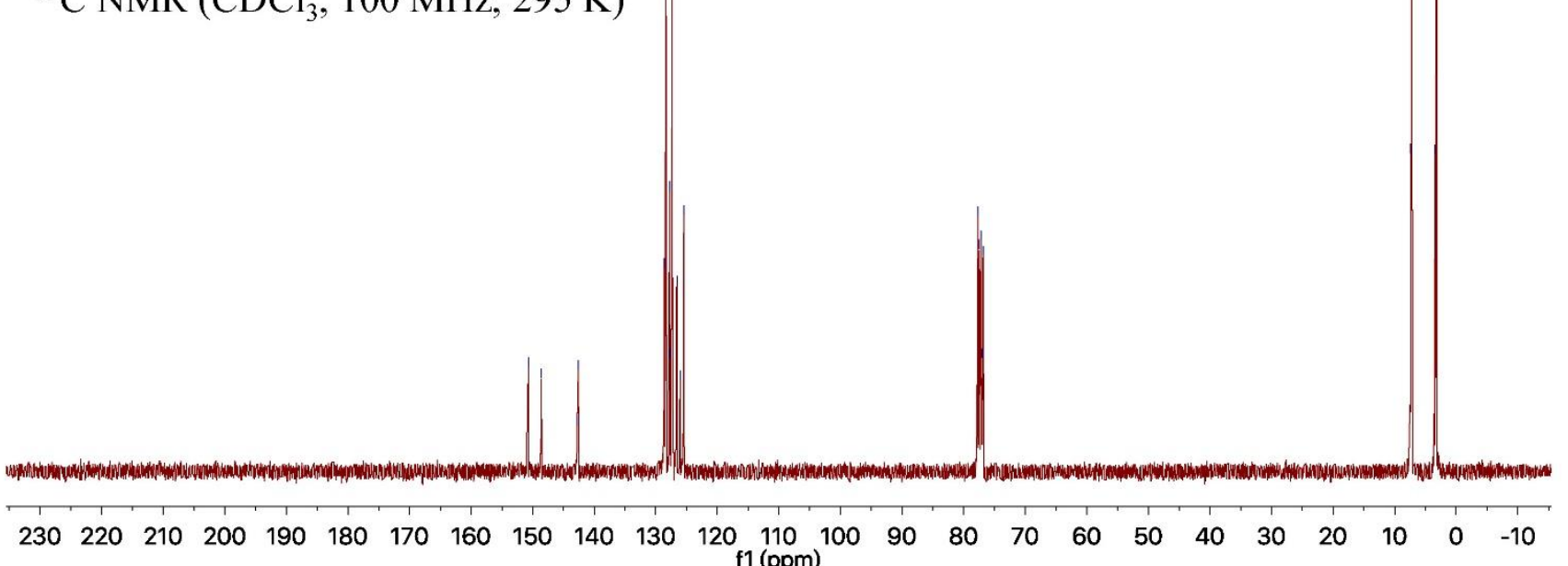

Figure S33. ${ }^{13} \mathrm{C}$ NMR spectrum of $\mathbf{P 8}$. 


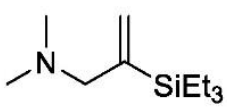

${ }^{1} \mathrm{H} \mathrm{NMR}\left(\mathrm{CDCl}_{3}, 400 \mathrm{MHz}, 298 \mathrm{~K}\right)$

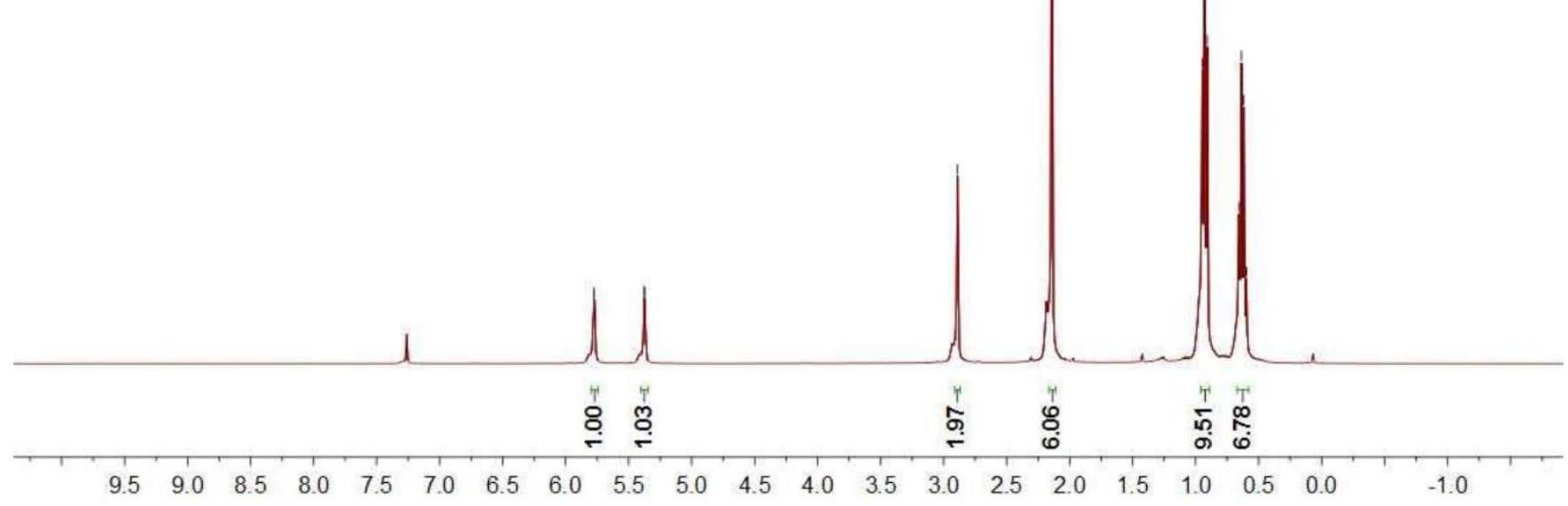

Figure S34. ${ }^{1} \mathrm{H}$ NMR spectrum of $\mathbf{P 9}$. 


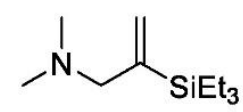

${ }^{13} \mathrm{C} \mathrm{NMR}\left(\mathrm{CDCl}_{3}, 100 \mathrm{MHz}, 295 \mathrm{~K}\right)$

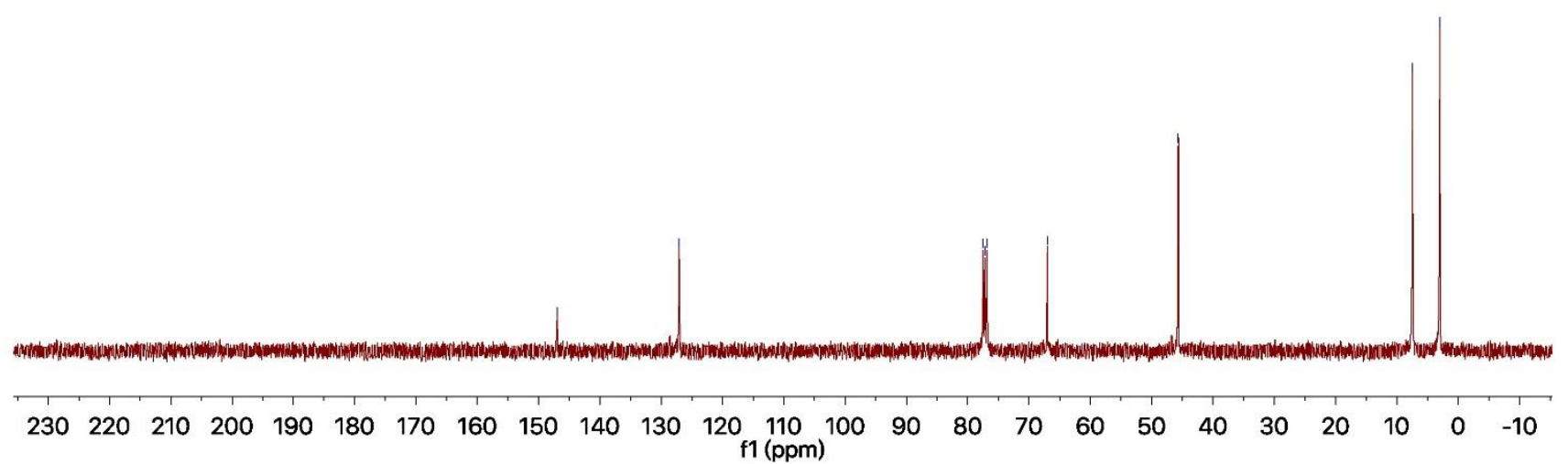

Figure S35. ${ }^{13} \mathrm{C}$ NMR spectrum of $\mathbf{P 9}$. 


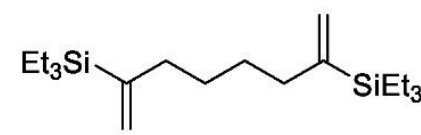

'H NMR (CDCl 3,400 MHz, 298 K)

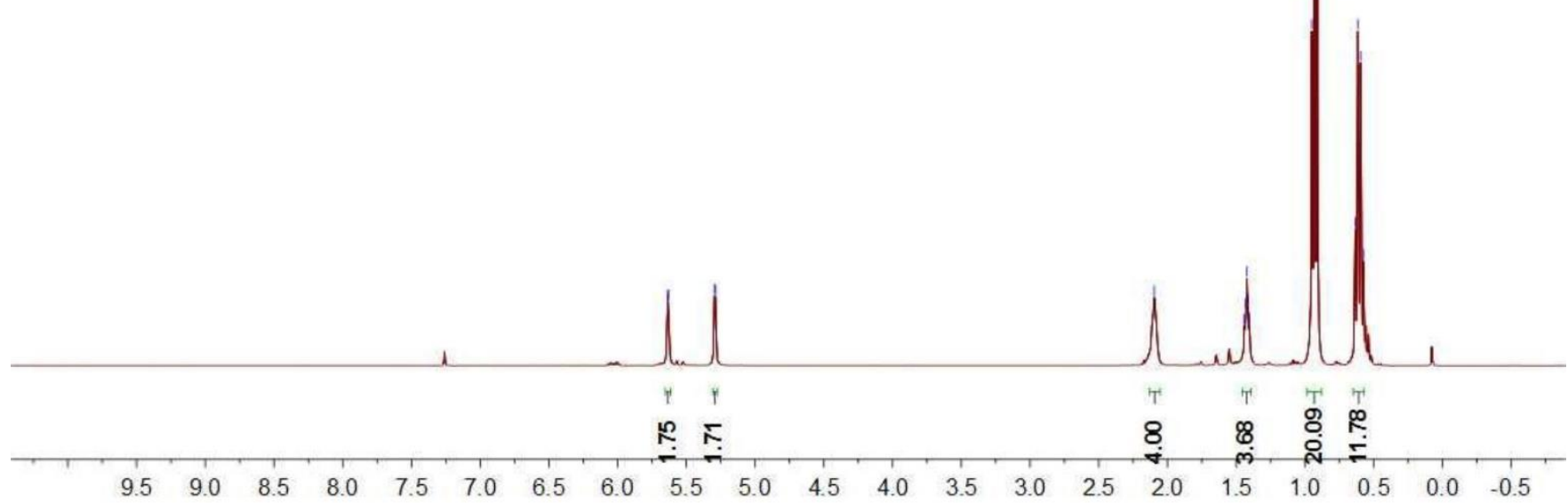

Figure S36. ${ }^{1} \mathrm{H}$ NMR spectrum of $\mathbf{P 1 0}$. 

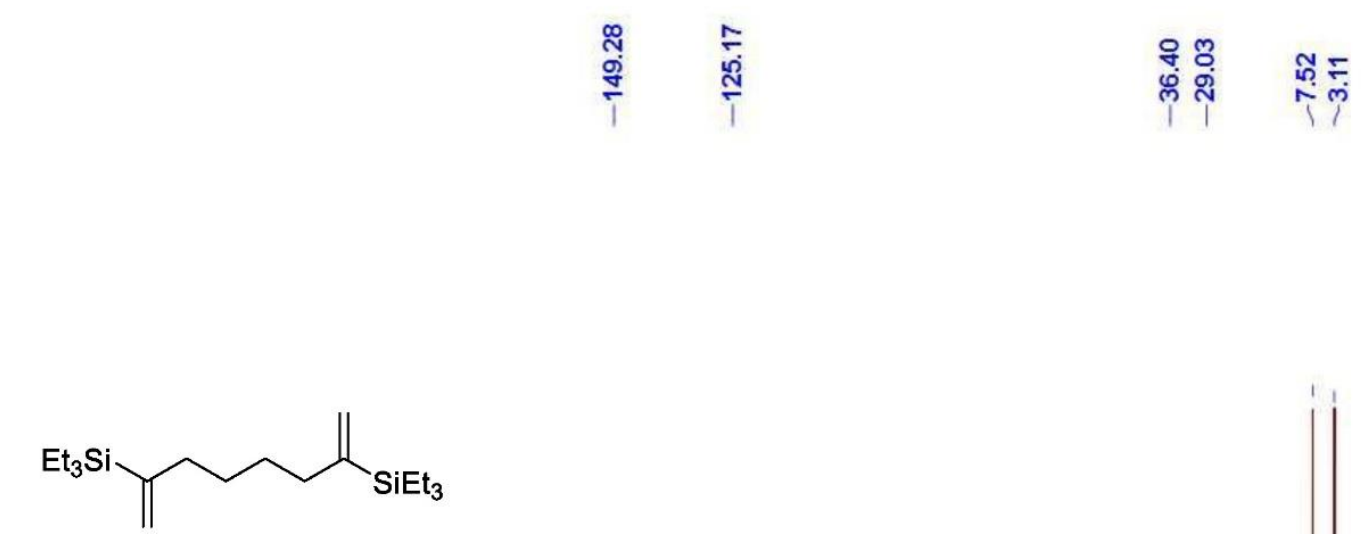

${ }^{13} \mathrm{C} \mathrm{NMR}\left(\mathrm{CDCl}_{3}, 100 \mathrm{MHz}, 298 \mathrm{~K}\right)$

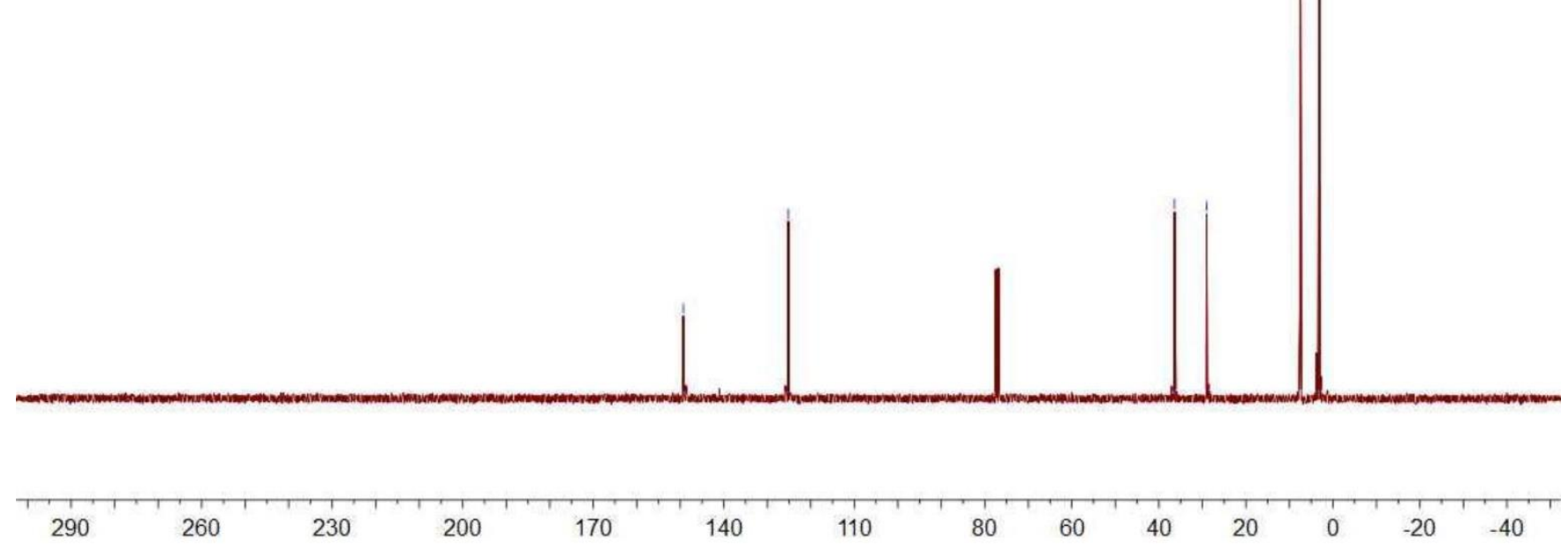

Figure S37. ${ }^{13} \mathrm{C}$ NMR spectrum of P10. 


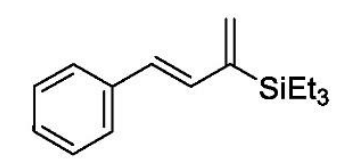

${ }^{1} \mathrm{H}$ NMR $\left(\mathrm{CDCl}_{3}, 400 \mathrm{MHz}, 293 \mathrm{~K}\right)$

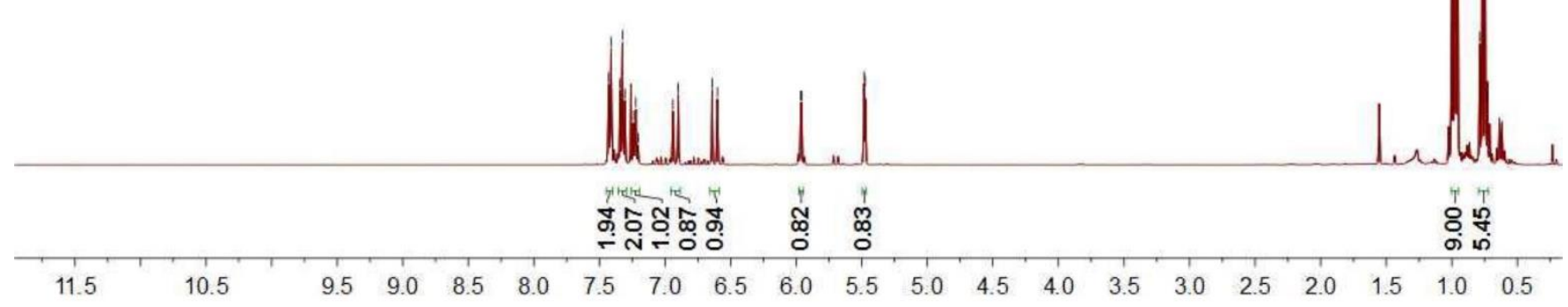

Figure S38. ${ }^{1} \mathrm{H}$ NMR spectrum of $\mathbf{P 1 1 .}$ 

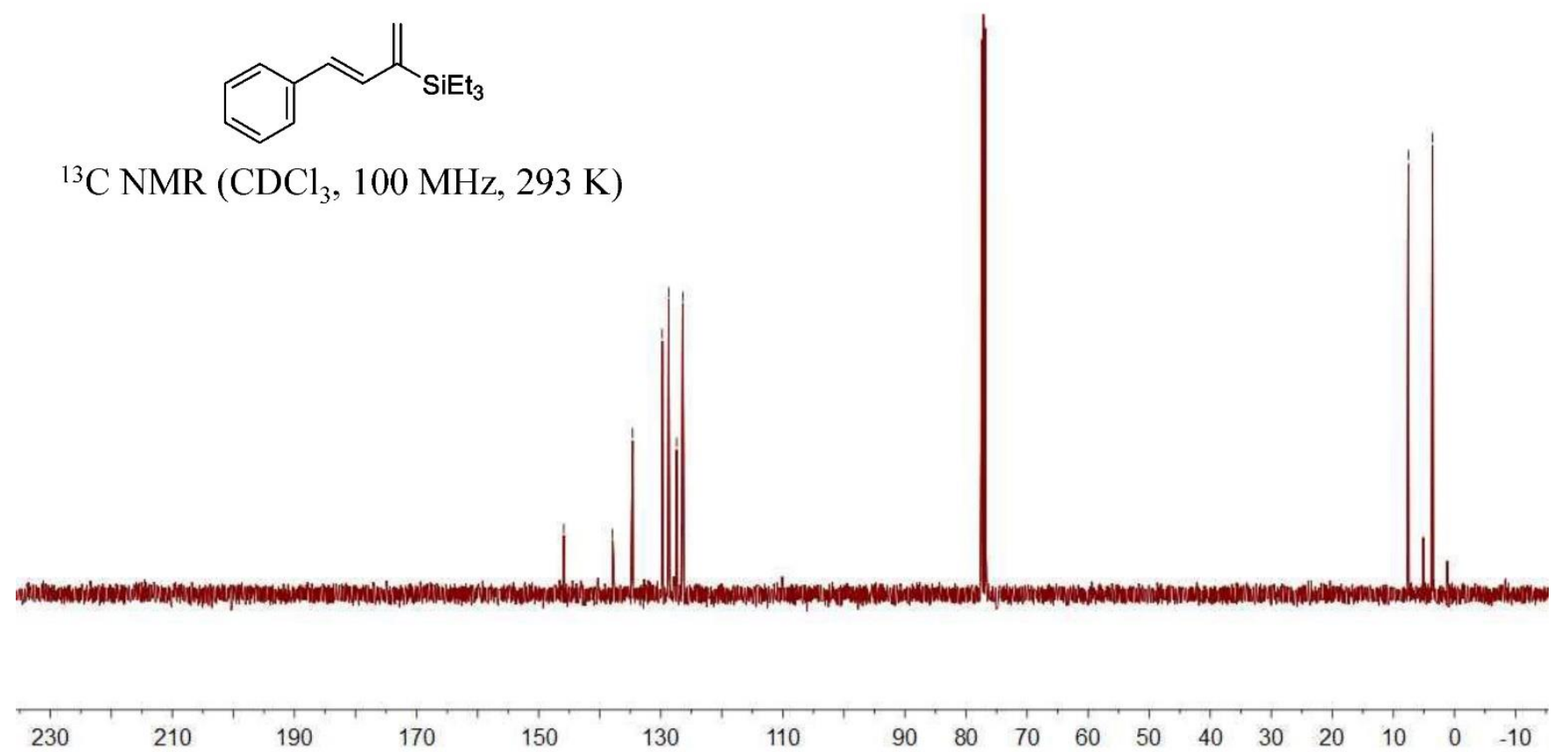

Figure S39. ${ }^{13} \mathrm{C}$ NMR spectrum of $\mathbf{P 1 1}$. 


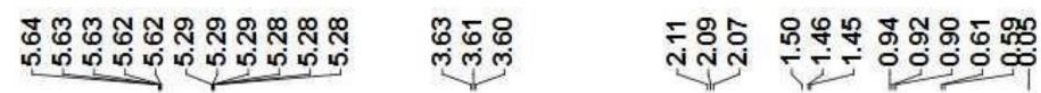

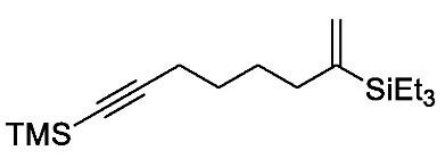

'H NMR ( $\mathrm{CDCl}_{3}, 400$ MHz, $\left.296 \mathrm{~K}\right)$

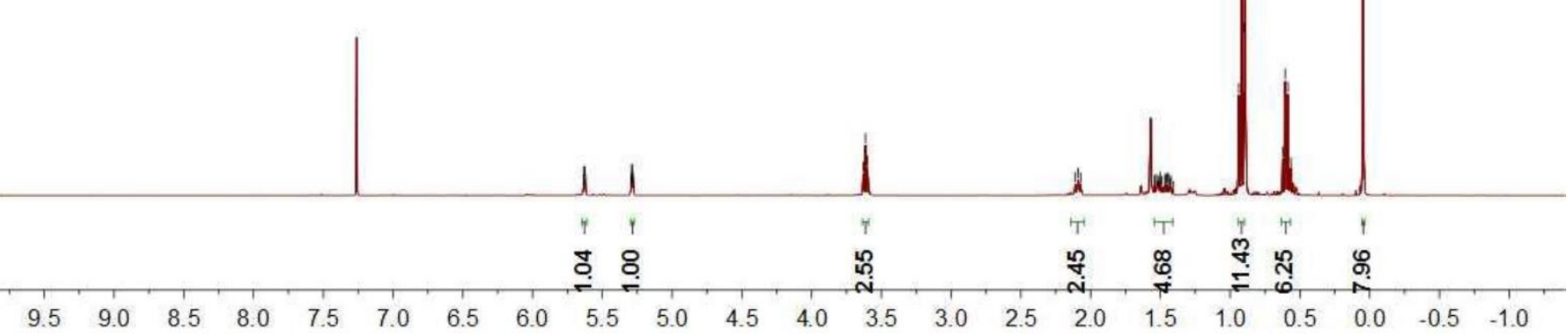

Figure S40. ${ }^{1} \mathrm{H}$ NMR spectrum of P12. 

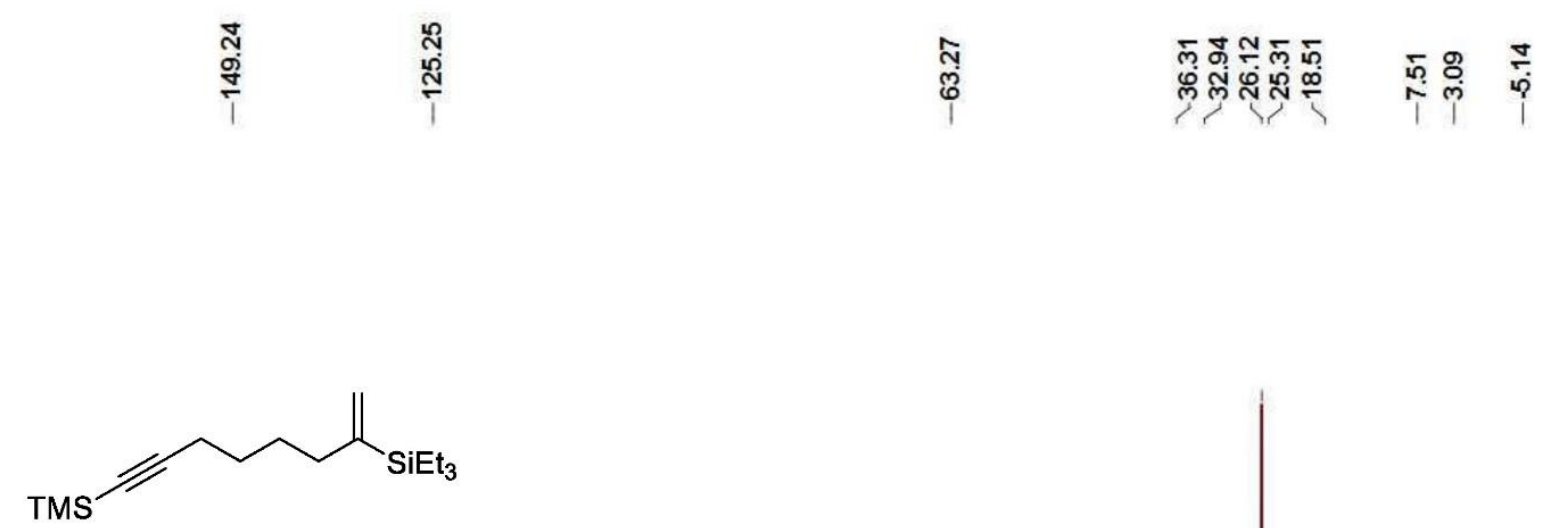

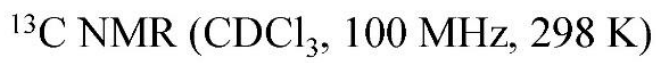
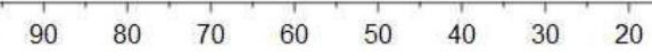

Figure S41. ${ }^{13} \mathrm{C}$ NMR spectrum of P12. 


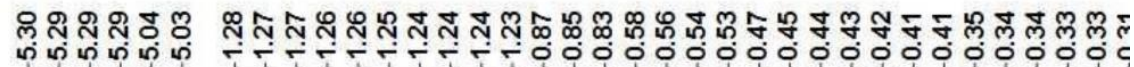

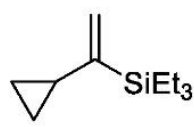

${ }^{1} \mathrm{H} \mathrm{NMR}\left(\mathrm{CDCl}_{3}, 400 \mathrm{MHz}, 298 \mathrm{~K}\right)$

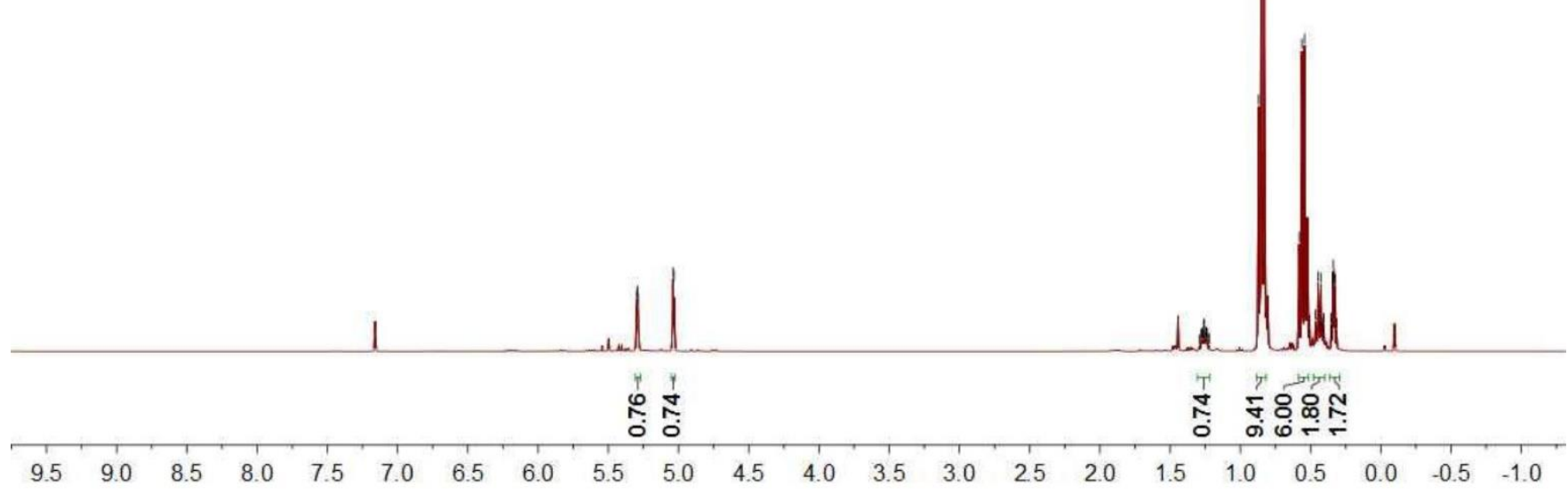

Figure S42. ${ }^{1} \mathrm{H}$ NMR spectrum of P13. 


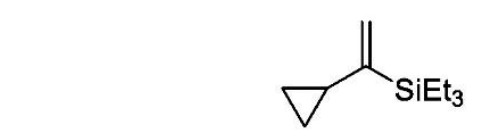

${ }^{13} \mathrm{C} \mathrm{NMR}\left(\mathrm{CDCl}_{3}, 100 \mathrm{MHz}, 293 \mathrm{~K}\right)$
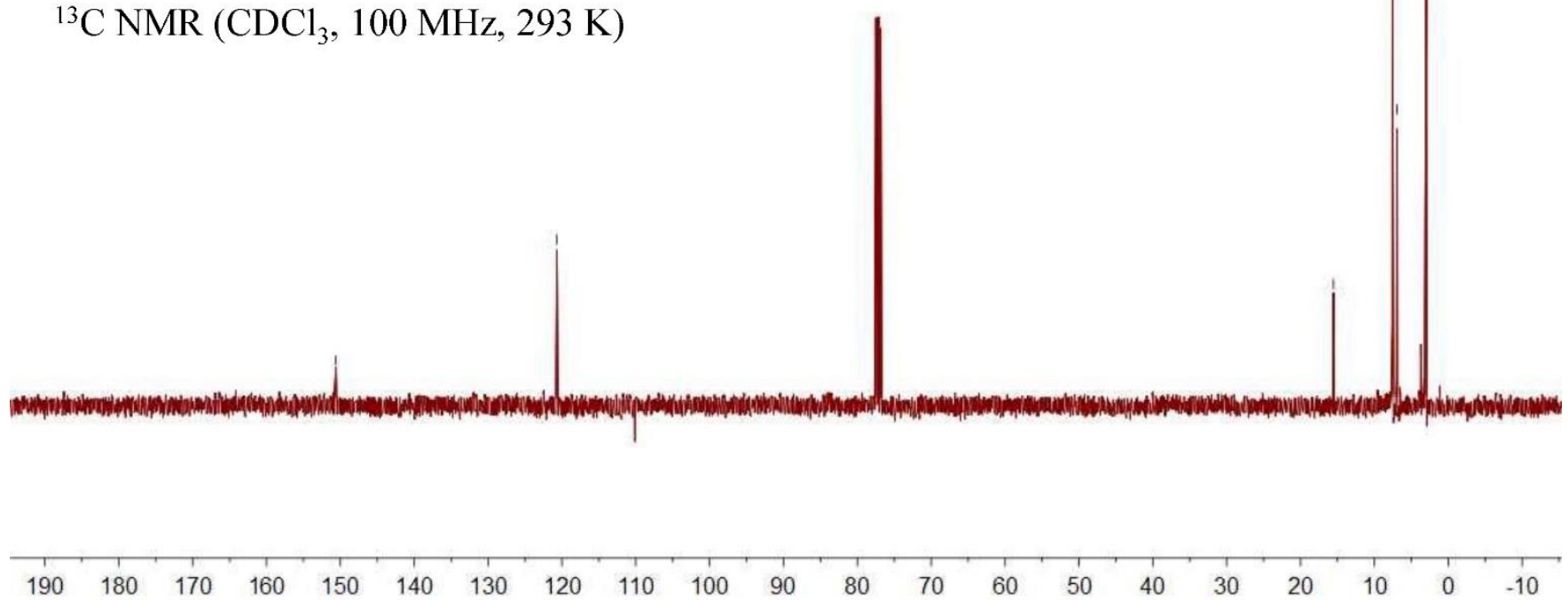

Figure S43. ${ }^{13} \mathrm{C}$ NMR spectrum of P13. 


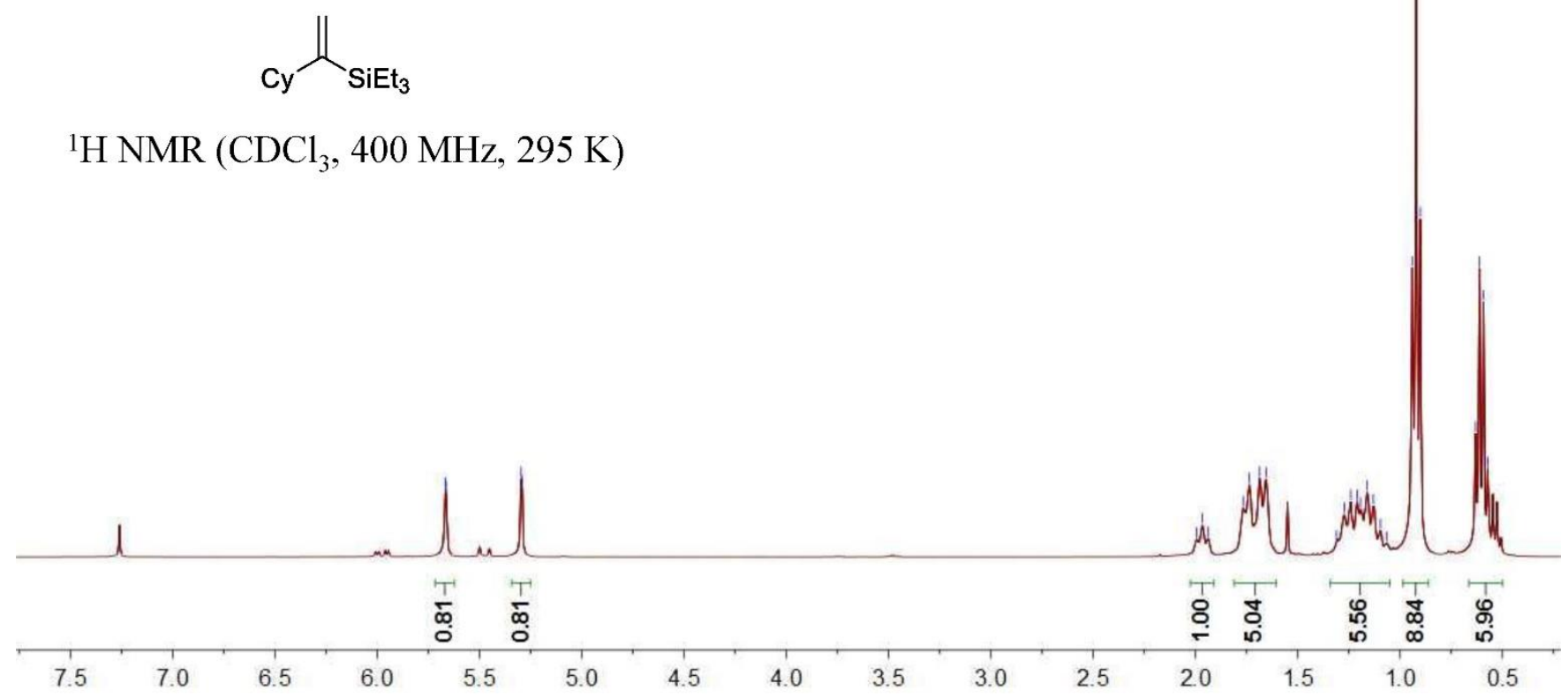

Figure S44. ${ }^{1} \mathrm{H}$ NMR spectrum of P14. 


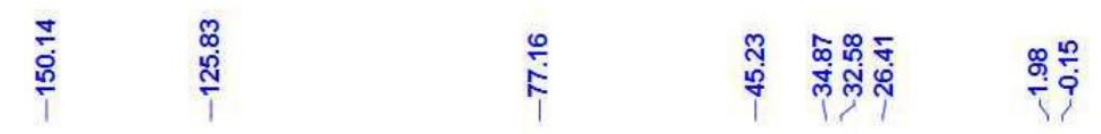

$$
\text { Cy } \stackrel{\mathrm{SiEt}}{3}_{\mathrm{S}}
$$

${ }^{13} \mathrm{C} \mathrm{NMR}\left(\mathrm{CDCl}_{3}, 100 \mathrm{MHz}, 295 \mathrm{~K}\right)$

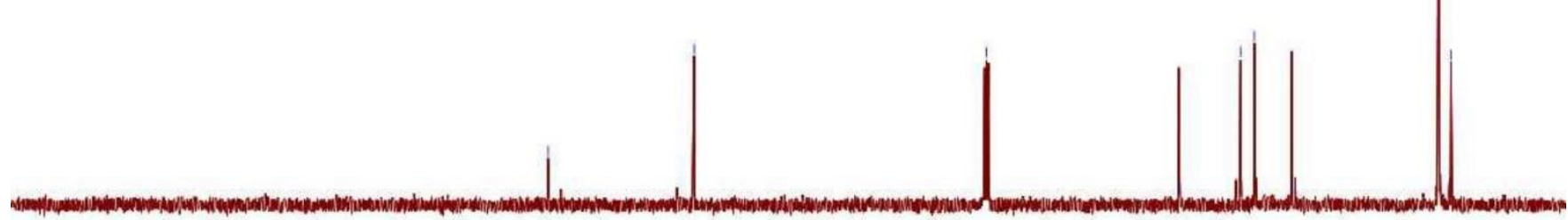

Figure S45. ${ }^{13} \mathrm{C}$ NMR spectrum of P14. 


$$
\text { Cy } \stackrel{\mathrm{SiEt}}{3}_{\mathrm{H}}
$$

${ }^{29} \mathrm{Si}$ NMR (79 MHz, $\mathrm{CDCl}_{3}, 297 \mathrm{~K}$ )

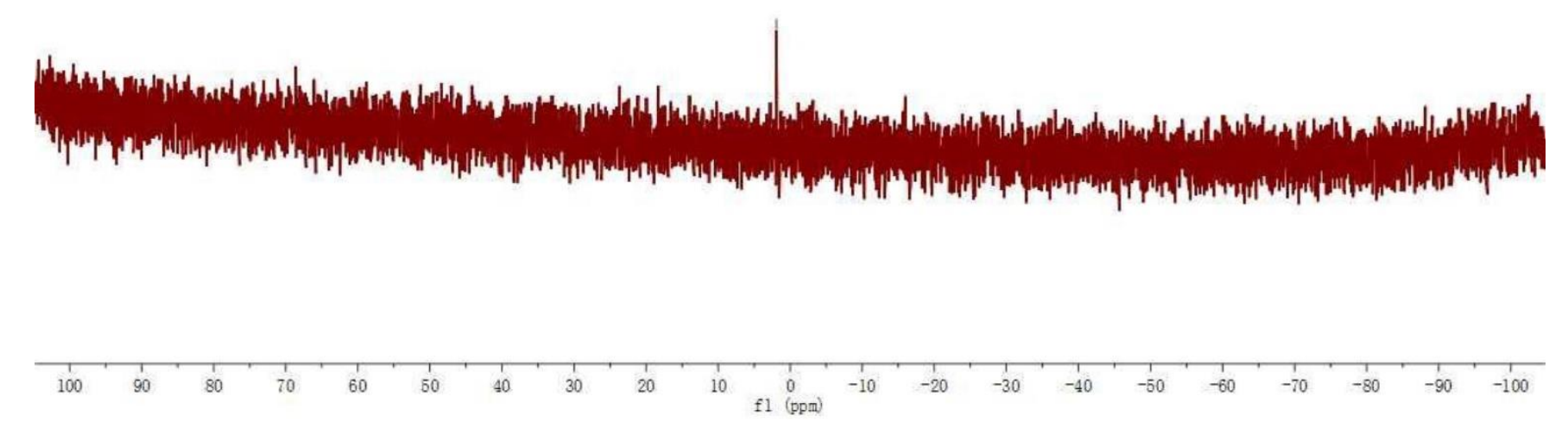

Figure S46. ${ }^{29}$ Si NMR spectrum of P14. 
${ }^{1} \mathrm{H}$ NMR (400 MHz, $\left.\mathrm{CDCl}_{3}, 298 \mathrm{~K}\right)$

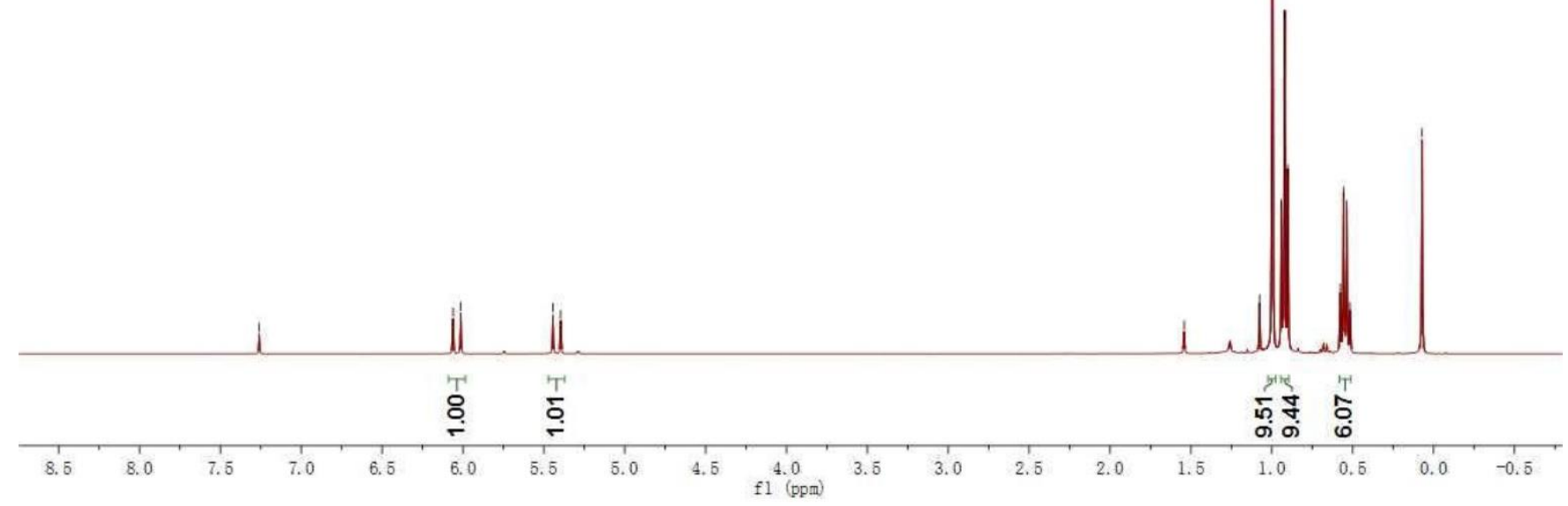

Figure S47. ${ }^{1} \mathrm{H}$ NMR spectrum of $\mathbf{P 1 5}$. 


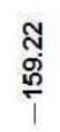

$\underset{\substack{\infty \\ \stackrel{\infty}{*}}}{\stackrel{\infty}{\infty}}$

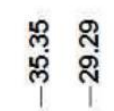

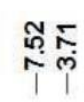<smiles>CC[SiH2]C=CC(C)(C)C</smiles>

${ }^{13} \mathrm{C}$ NMR (100 MHz, $\left.\mathrm{CDCl}_{3}, 296 \mathrm{~K}\right)$
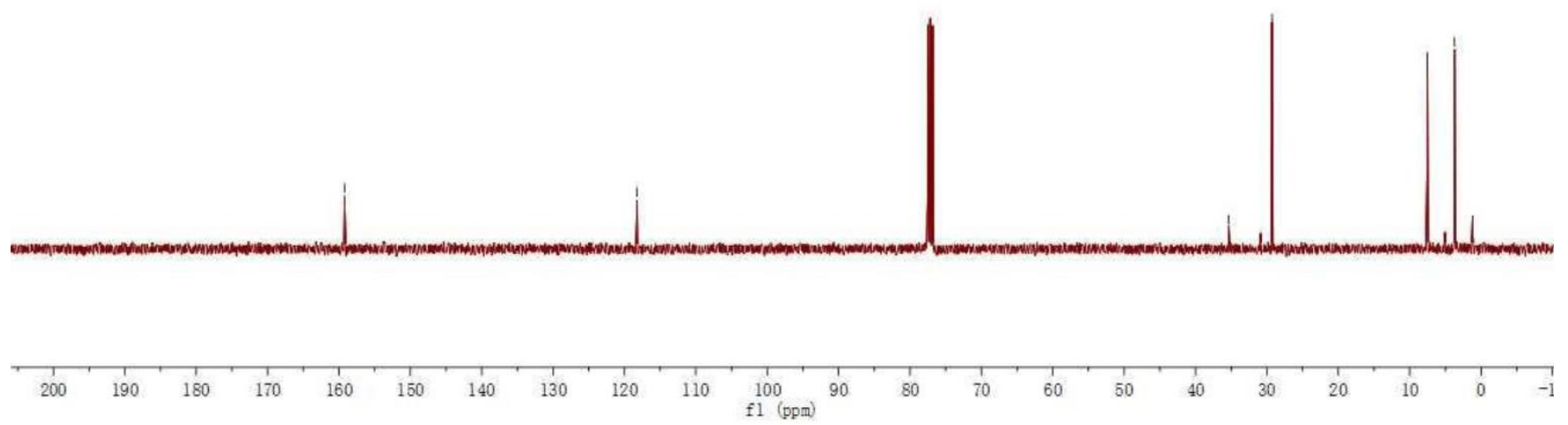

Figure S48. ${ }^{13} \mathrm{C}$ NMR spectrum of $\mathbf{P 1 5}$.

S75 


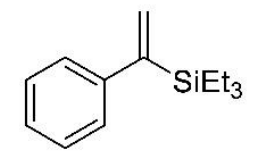

${ }^{1} \mathrm{H}$ NMR (400 MHz, $\left.\mathrm{CDCl}_{3}, 296 \mathrm{~K}\right)$

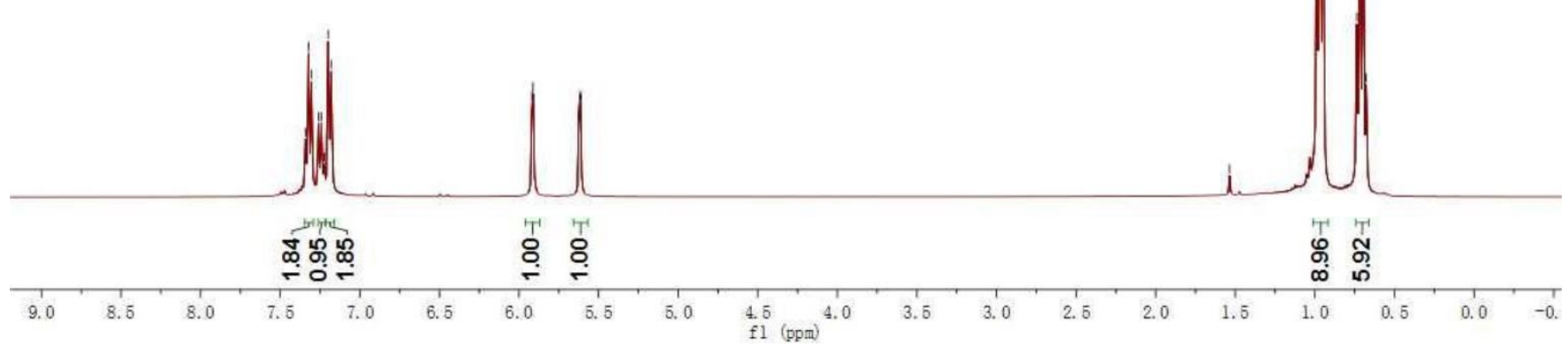

Figure S49. ${ }^{1} \mathrm{H}$ NMR spectrum of P16. 


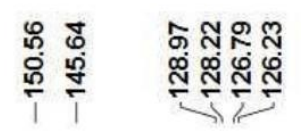

量贲<smiles>C=C([SiH2]CC)c1ccccc1</smiles>

${ }^{13} \mathrm{C}$ NMR (100 MHz, $\left.\mathrm{CDCl}_{3}, 295 \mathrm{~K}\right)$

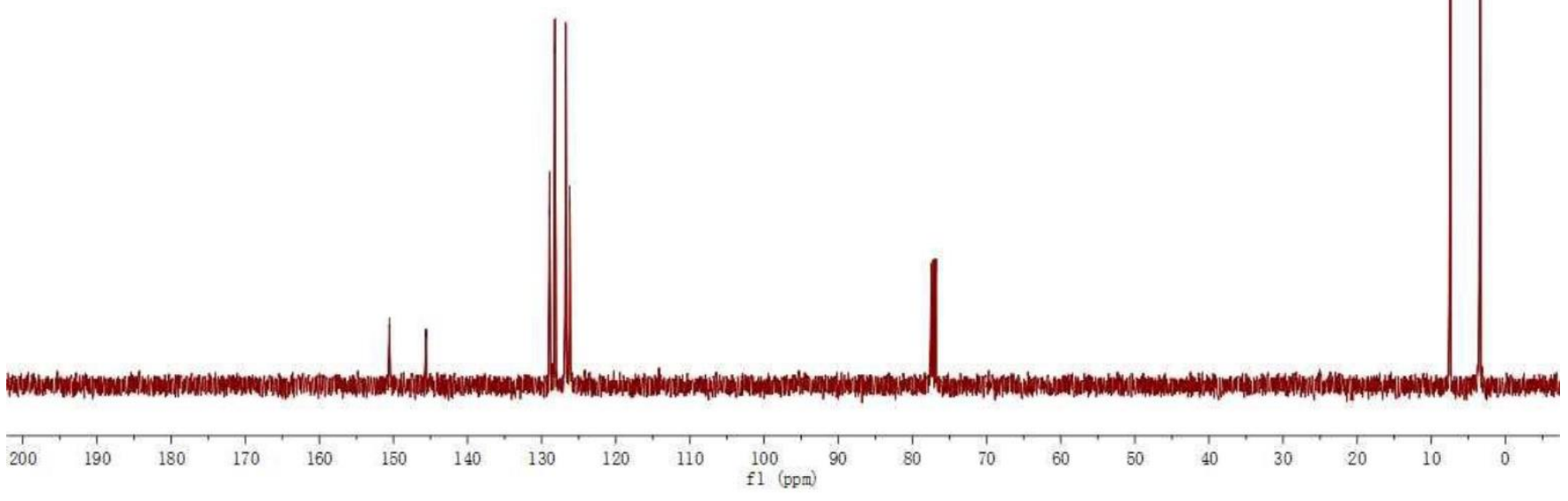

Figure S50. ${ }^{13} \mathrm{C}$ NMR spectrum of $\mathbf{P 1 6}$.

S77 


\section{ุำ}

NiNito

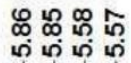

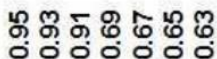

/1
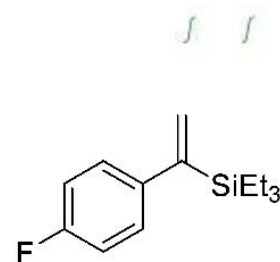

${ }^{1} \mathrm{H}$ NMR (400 MHz, $\left.\mathrm{CDCl}_{3}, 298 \mathrm{~K}\right)$

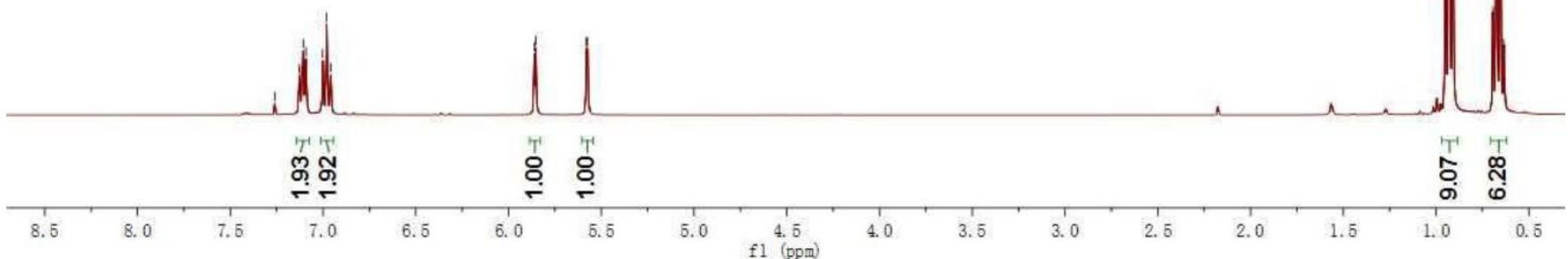

Figure S51. ${ }^{1} \mathrm{H}$ NMR spectrum of P17. 


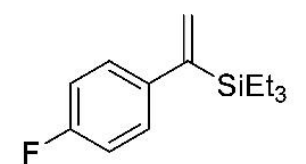

${ }^{13} \mathrm{C}$ NMR $\left(100 \mathrm{MHz}, \mathrm{CDCl}_{3}, 296 \mathrm{~K}\right)$

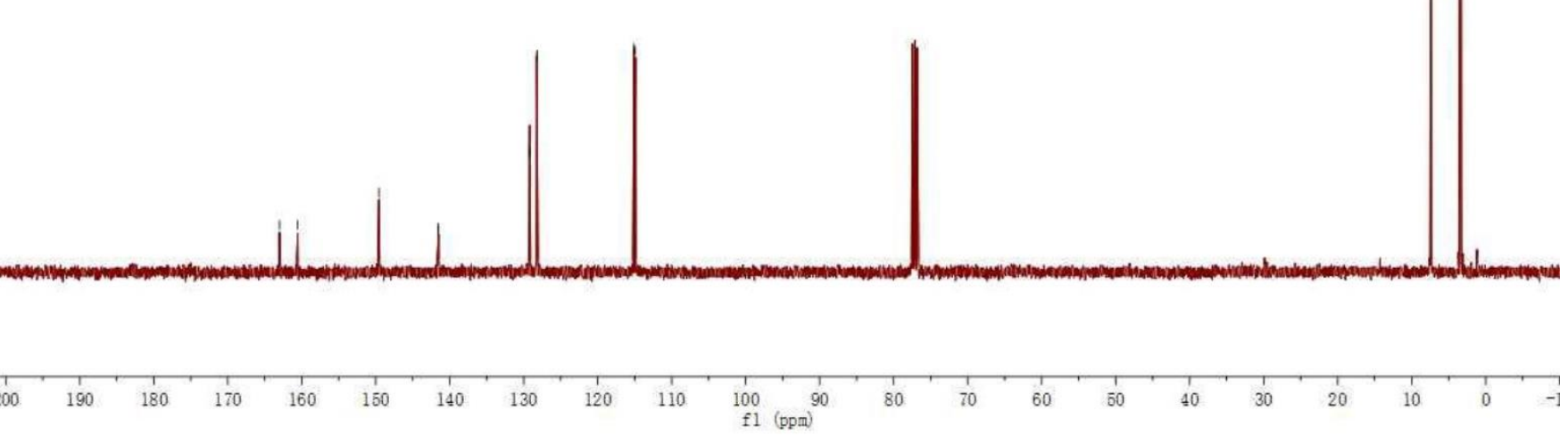

Figure S52. ${ }^{13} \mathrm{C}$ NMR spectrum of P17. 


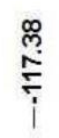

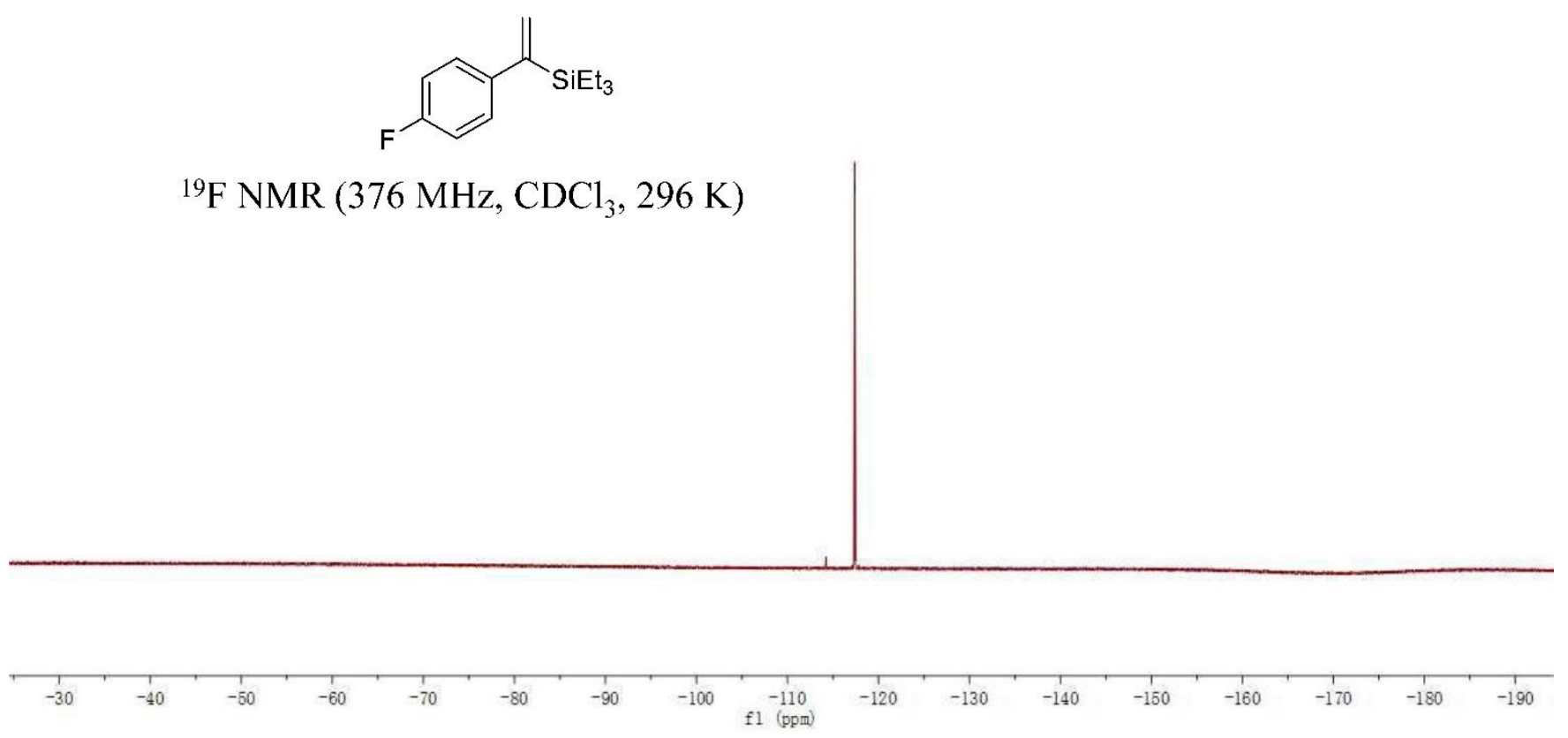

Figure S53. ${ }^{19}$ F NMR spectrum of P17.

S80 


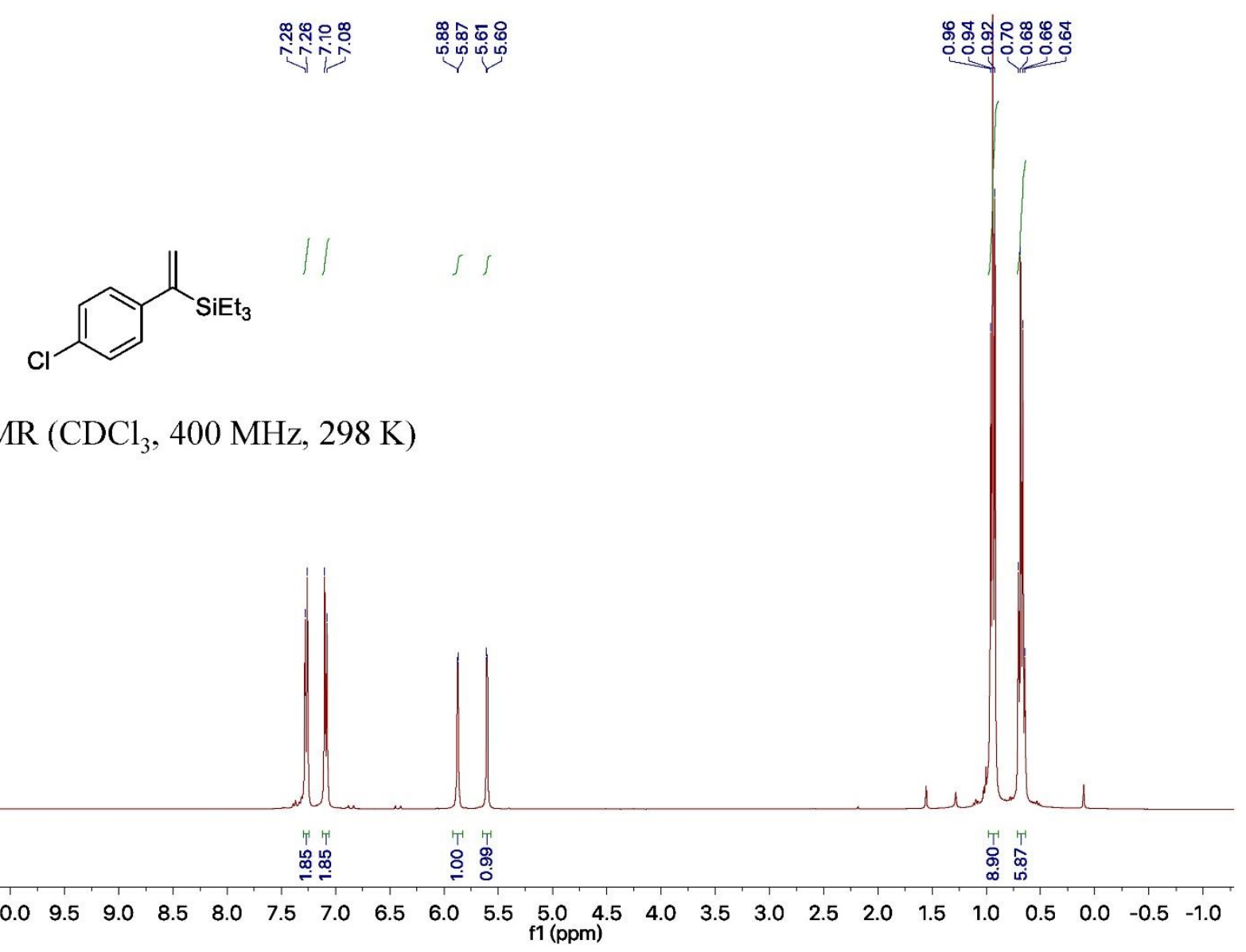

Figure S54. ${ }^{1} \mathrm{H}$ NMR spectrum of P18.

581 


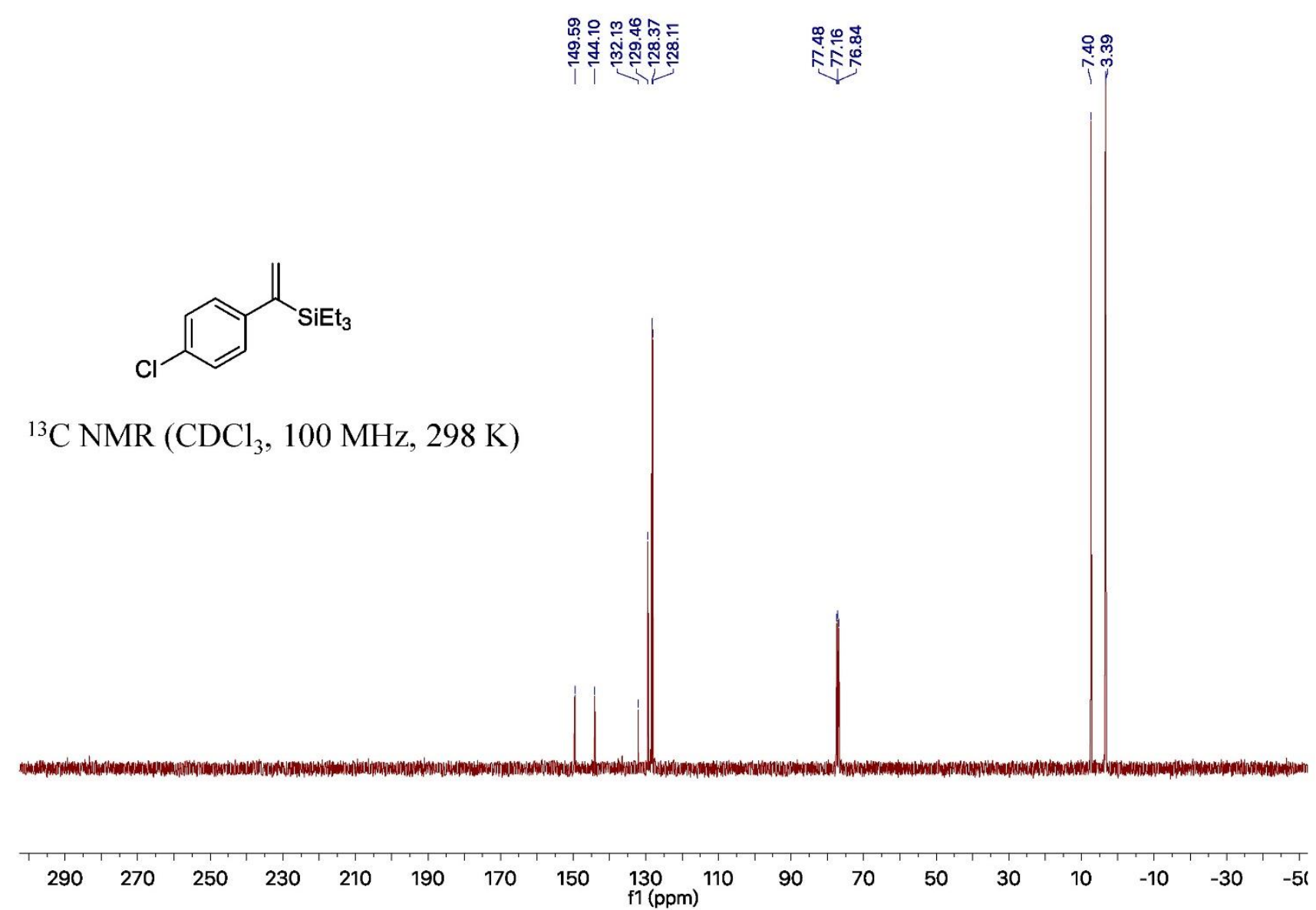

Figure S55. ${ }^{13} \mathrm{C}$ NMR spectrum of $\mathbf{P 1 8}$. 


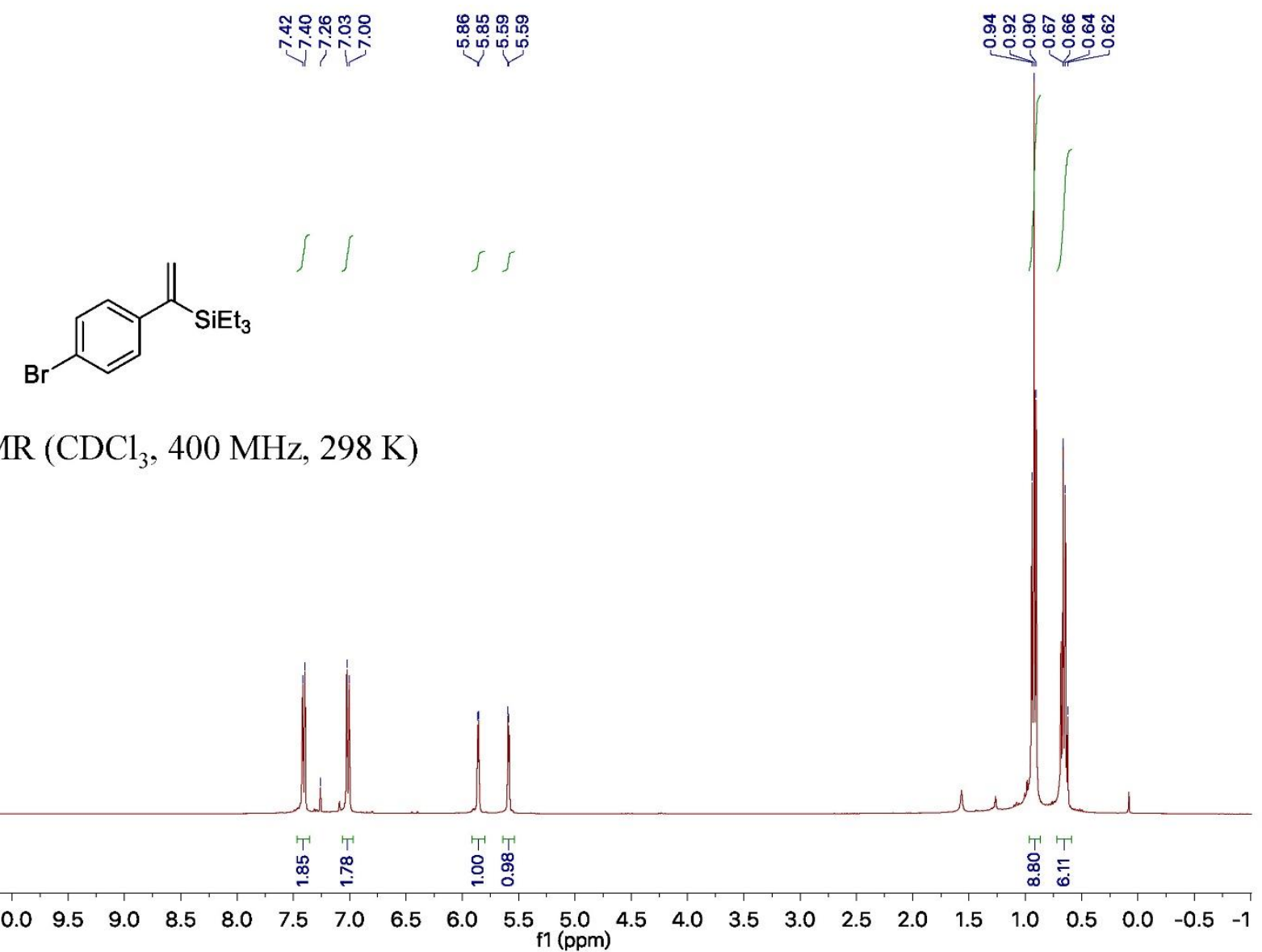

Figure S56. ${ }^{1} \mathrm{H}$ NMR spectrum of P19. 

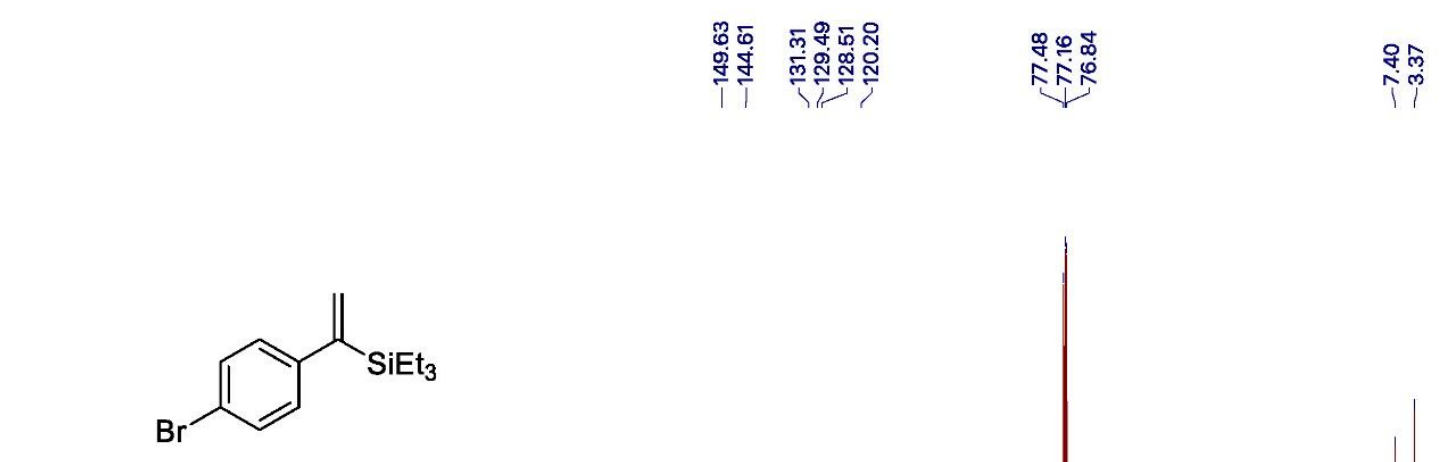

${ }^{13} \mathrm{C} \mathrm{NMR}\left(\mathrm{CDCl}_{3}, 100 \mathrm{MHz}, 298 \mathrm{~K}\right)$
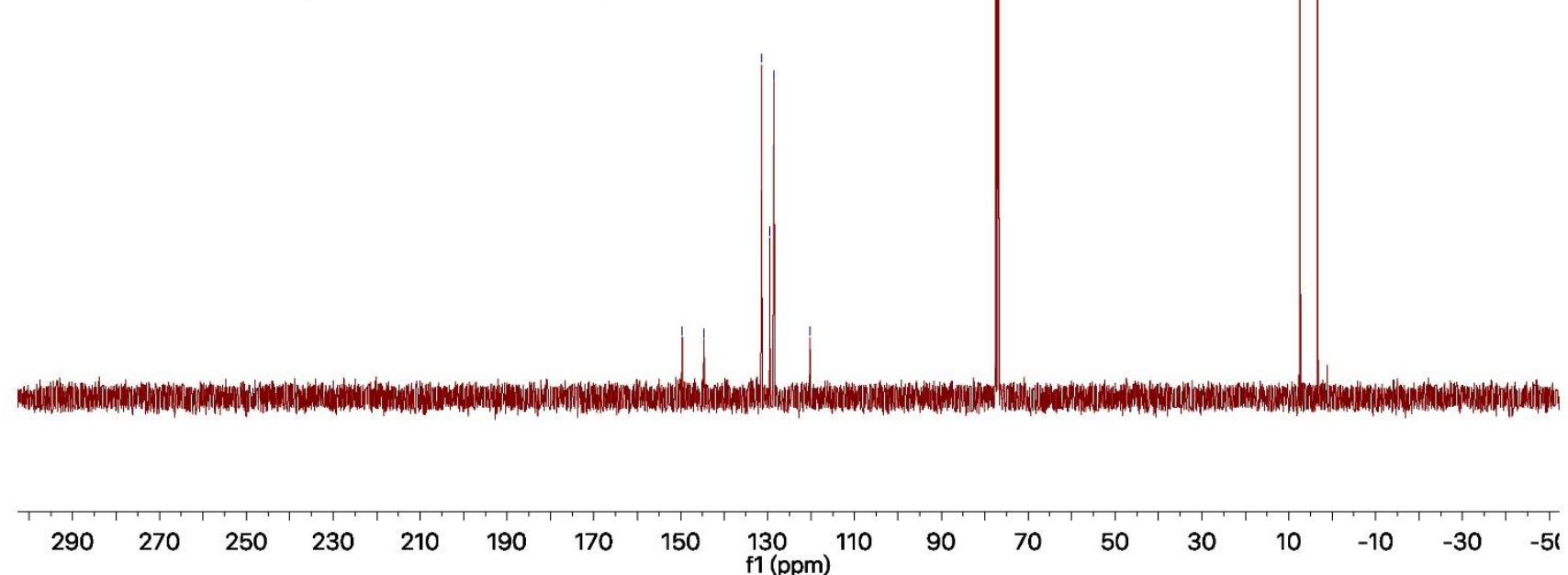

Figure S57. ${ }^{13} \mathrm{C}$ NMR spectrum of $\mathbf{P 1 9}$. 


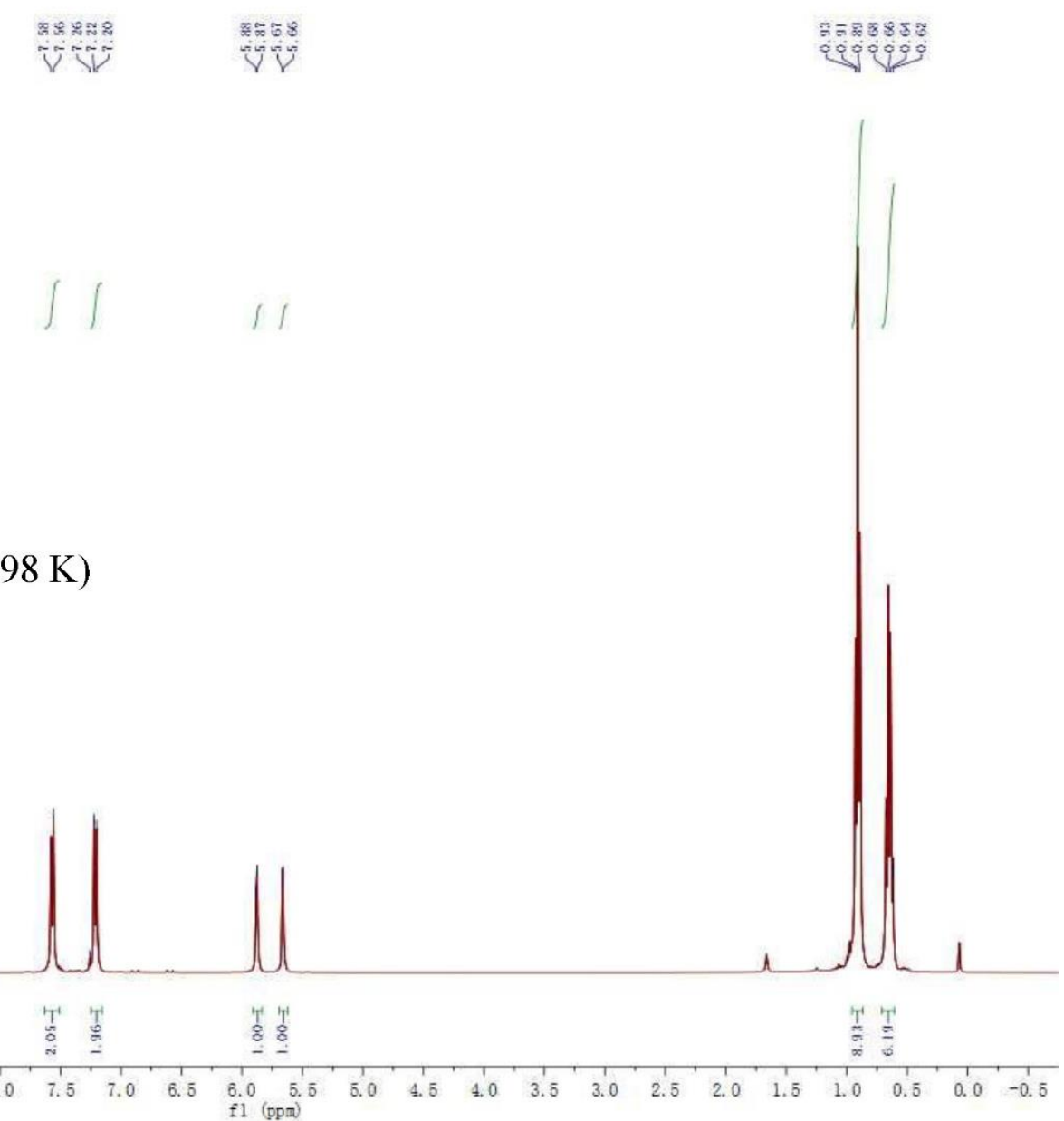

Figure S58. ${ }^{1} \mathrm{H}$ NMR spectrum of $\mathbf{P 2 0}$.

585 


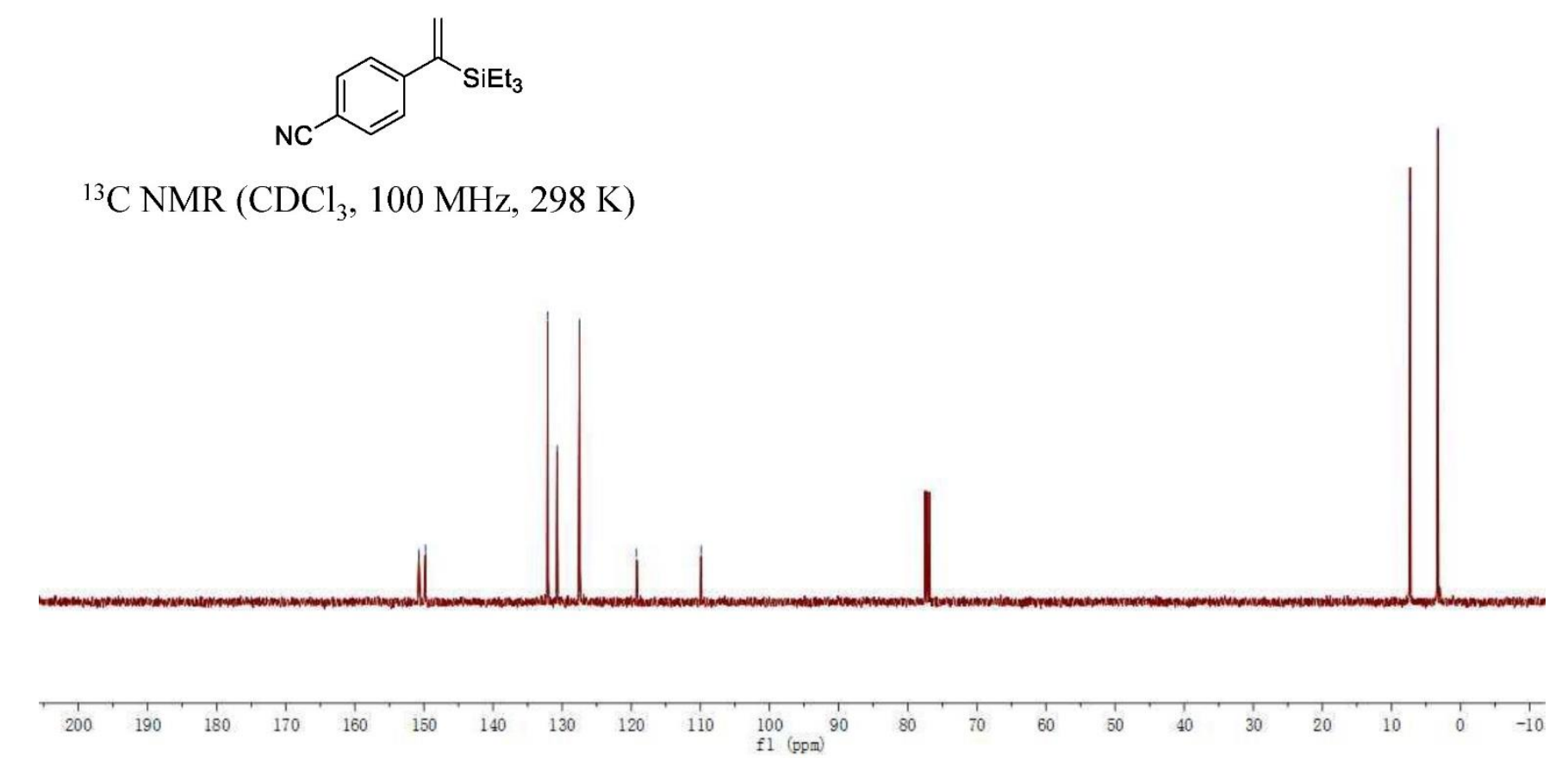

Figure S59. ${ }^{13} \mathrm{C}$ NMR spectrum of $\mathbf{P 2 0}$. 


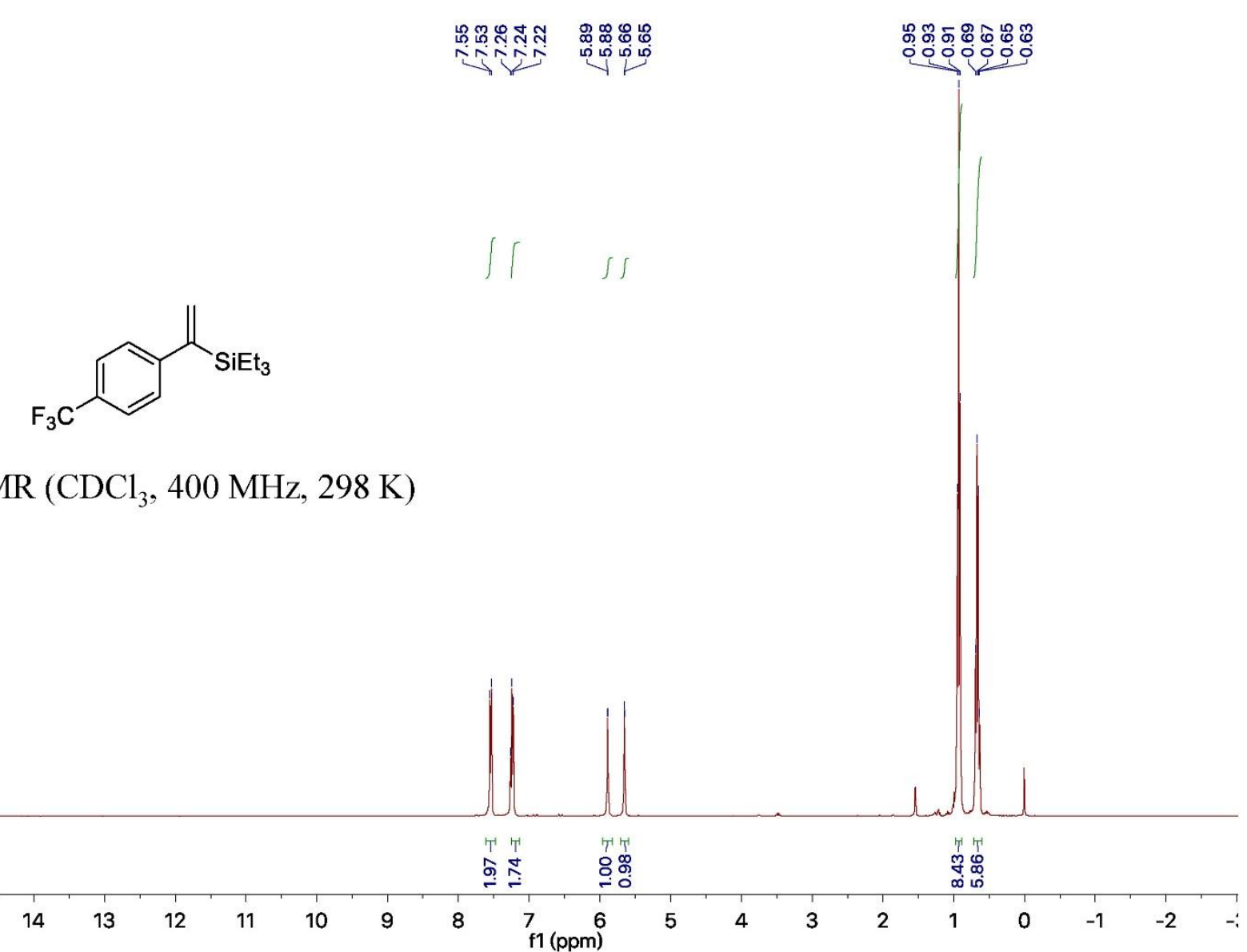

Figure S60. ${ }^{1} \mathrm{H}$ NMR spectrum of $\mathbf{P 2 1}$. 


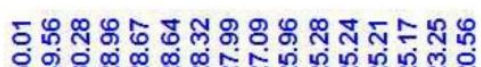

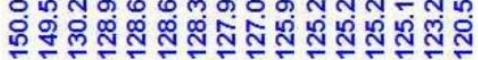

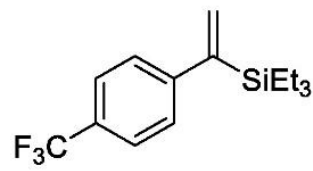

${ }^{13} \mathrm{C} \mathrm{NMR}\left(\mathrm{CDCl}_{3}, 100 \mathrm{MHz}, 298 \mathrm{~K}\right)$

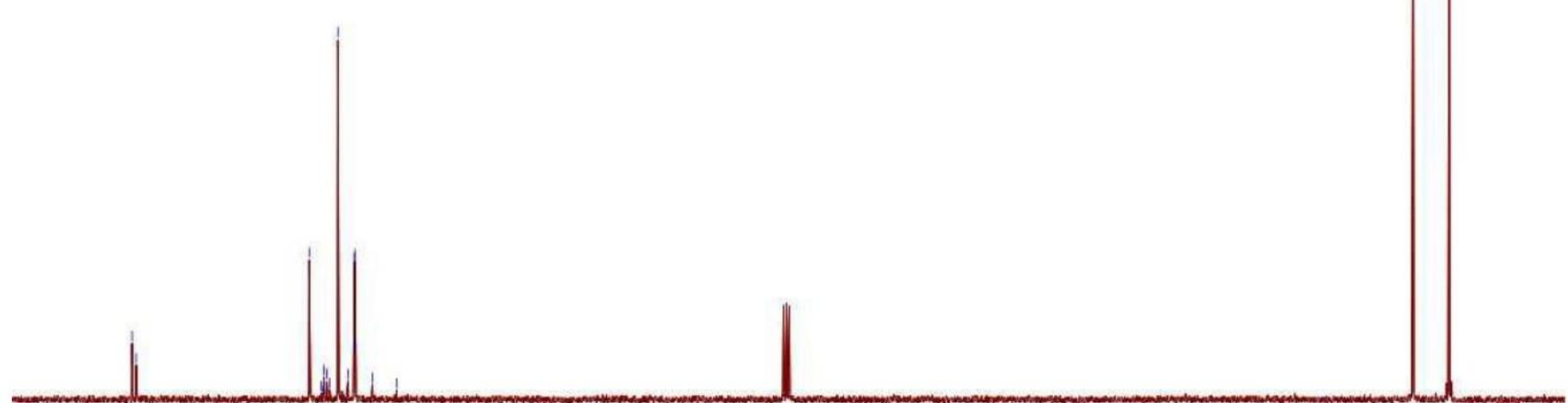

$\begin{array}{lll}160 \quad 150 & 140\end{array}$

Figure S61. ${ }^{13} \mathrm{C}$ NMR spectrum of $\mathbf{P 2 1}$. 


$$
\underline{-1}
$$




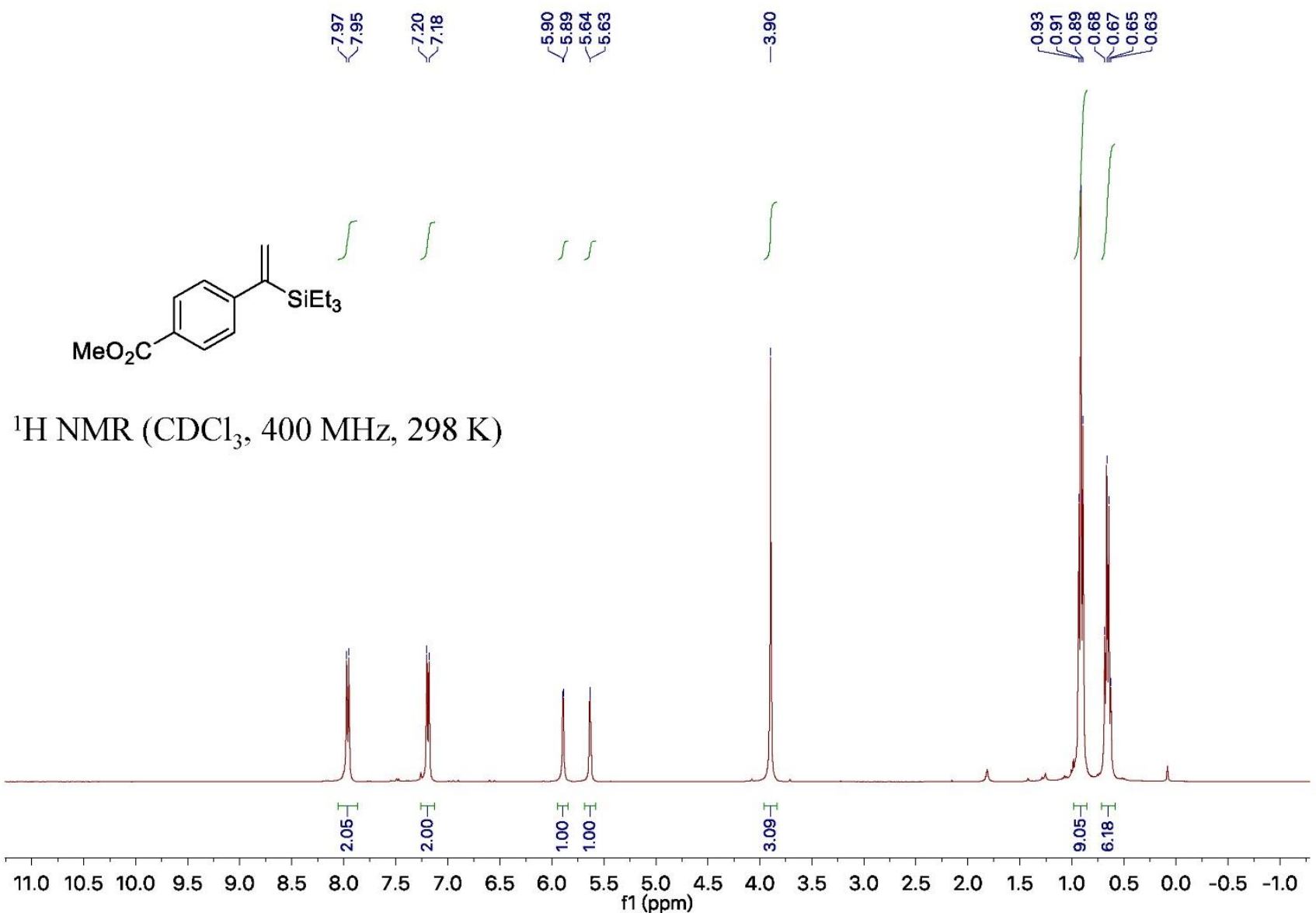

Figure S63. ${ }^{1} \mathrm{H}$ NMR spectrum of $\mathbf{P 2 2}$. 


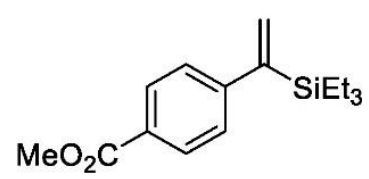

${ }^{13} \mathrm{C} \mathrm{NMR}\left(\mathrm{CDCl}_{3}, 100 \mathrm{MHz}, 298 \mathrm{~K}\right)$

$\begin{array}{lllllllllllllllllllllll}200 & 190 & 180 & 170 & 160 & 150 & 140 & 130 & 120 & 110 & 100 & 90 & 80 & 70 & 60 & 50 & 40 & 30 & 20 & 10 & 0 & -10\end{array}$

Figure S64. ${ }^{13} \mathrm{C}$ NMR spectrum of $\mathbf{P 2 2}$. 


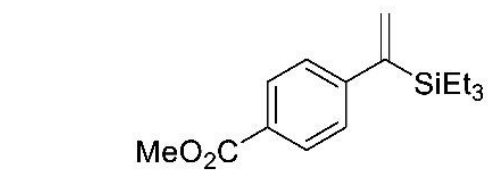

${ }^{29} \mathrm{Si}$ NMR (79 MHz, $\mathrm{CDCl}_{3}, 297 \mathrm{~K}$ )

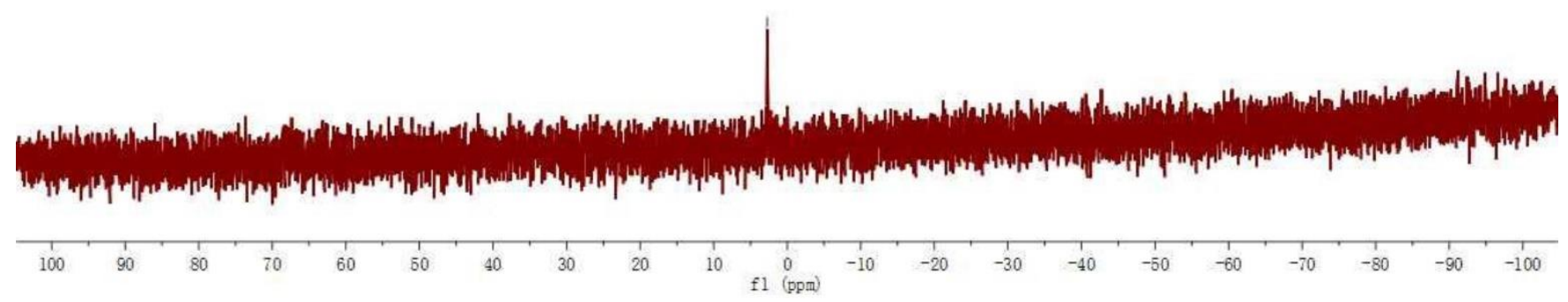

Figure S65. ${ }^{29}$ Si NMR spectrum of $\mathbf{P 2 2}$. 


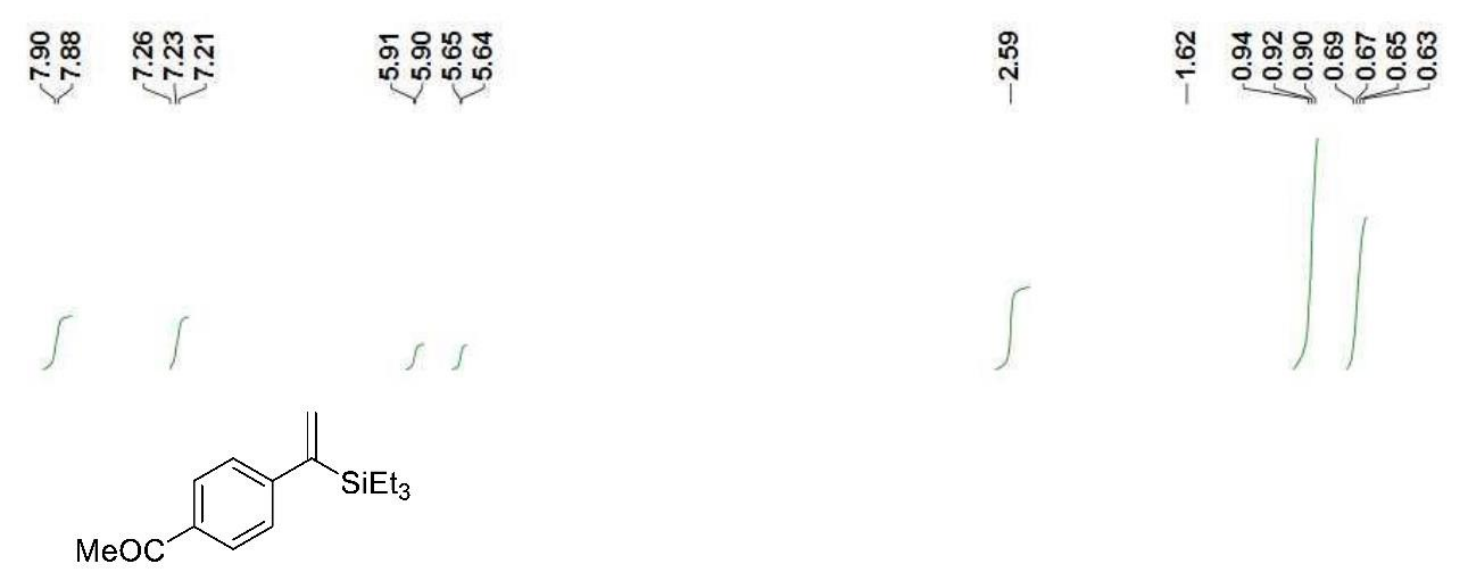

${ }^{1} \mathrm{H}$ NMR (400 MHz, $\mathrm{CDCl}_{3}, 298 \mathrm{~K}$ )

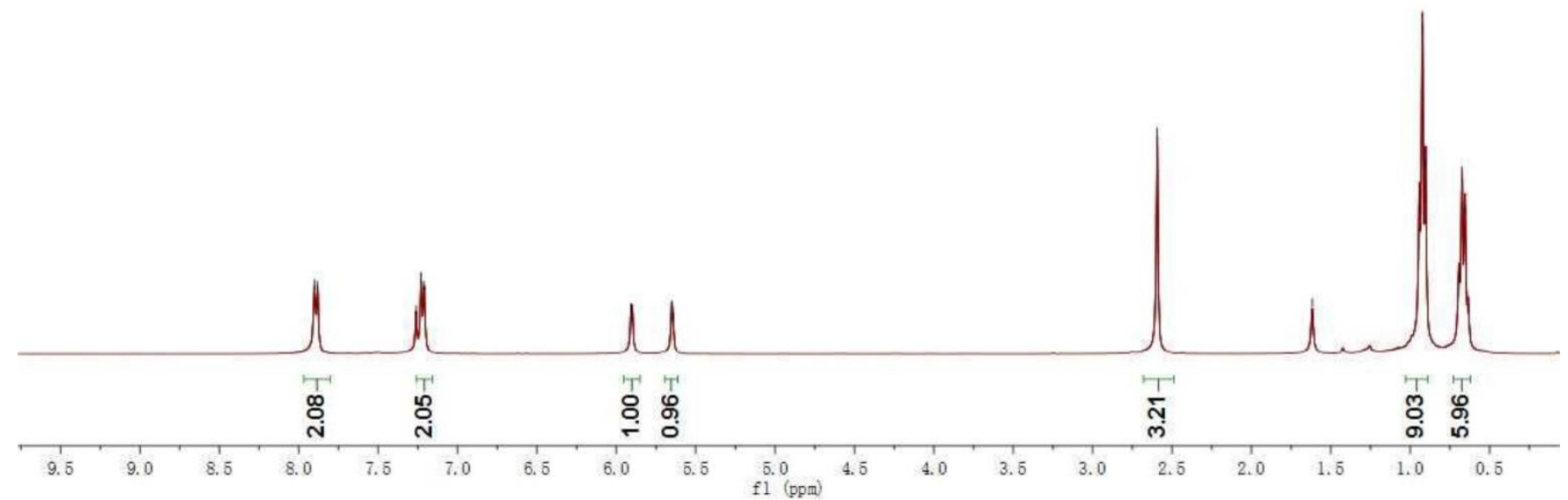

Figure S66. ${ }^{1} \mathrm{H}$ NMR spectrum of $\mathbf{P 2 3}$. 

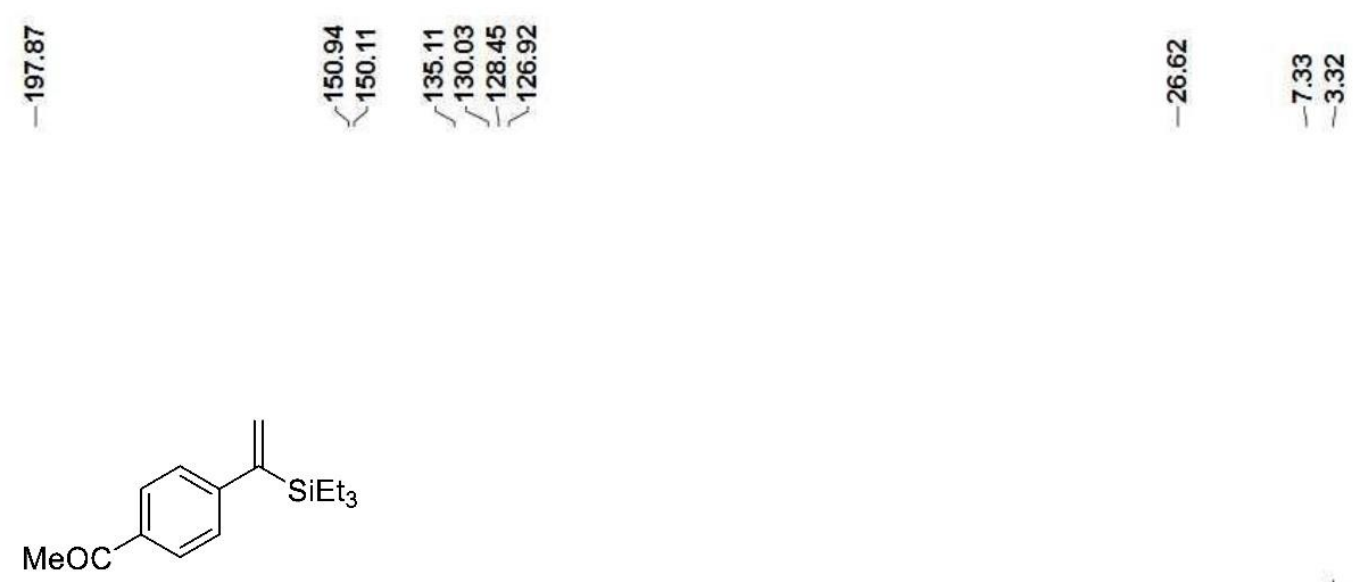

${ }^{13} \mathrm{C}$ NMR (100 MHz, $\mathrm{CDCl}_{3}, 297 \mathrm{~K}$ )
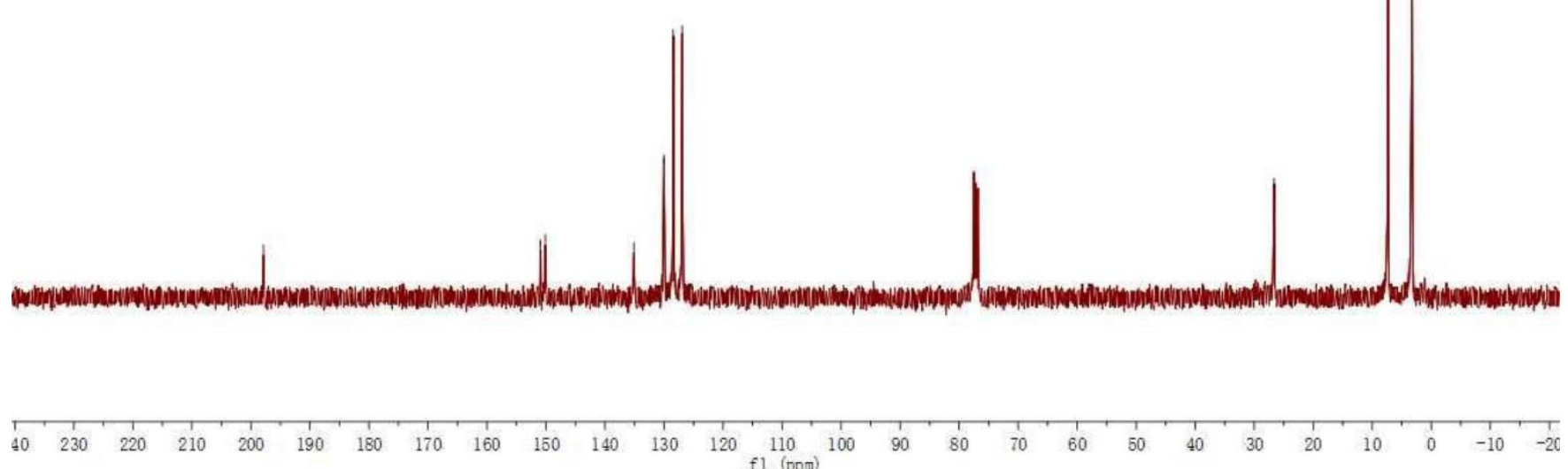

Figure S67. ${ }^{13} \mathrm{C}$ NMR spectrum of $\mathbf{P 2 3}$. 


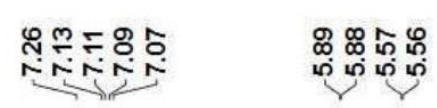
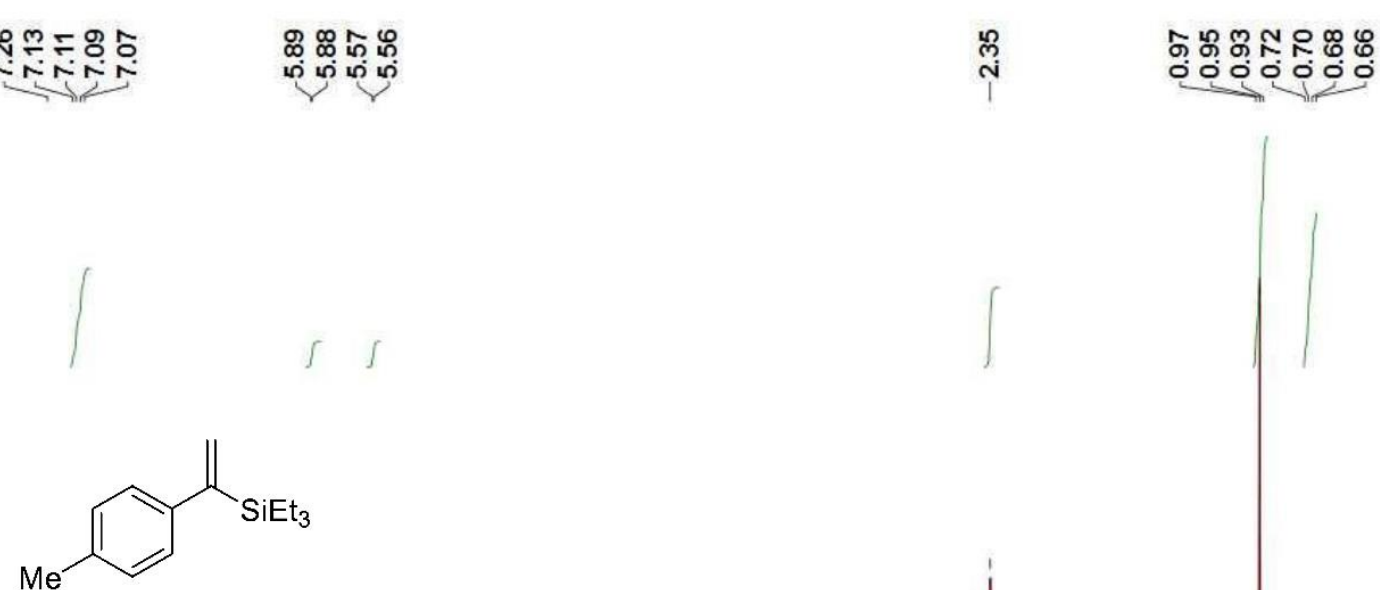

${ }^{1} \mathrm{H}$ NMR (400 MHz, $\left.\mathrm{CDCl}_{3}, 298 \mathrm{~K}\right)$

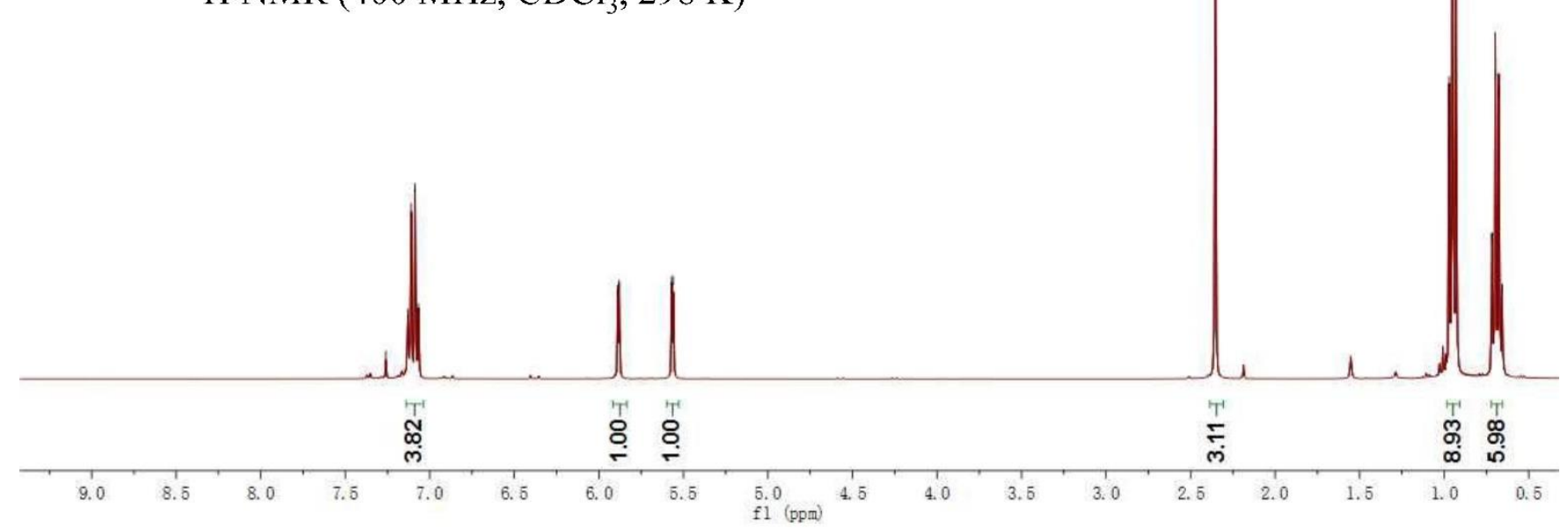

Figure S68. ${ }^{1} \mathrm{H}$ NMR spectrum of $\mathbf{P 2 4}$. 


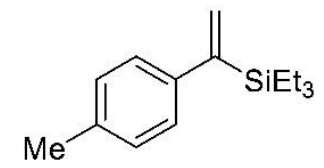

${ }^{13} \mathrm{C}$ NMR (100 MHz, $\left.\mathrm{CDCl}_{3}, 298 \mathrm{~K}\right)$

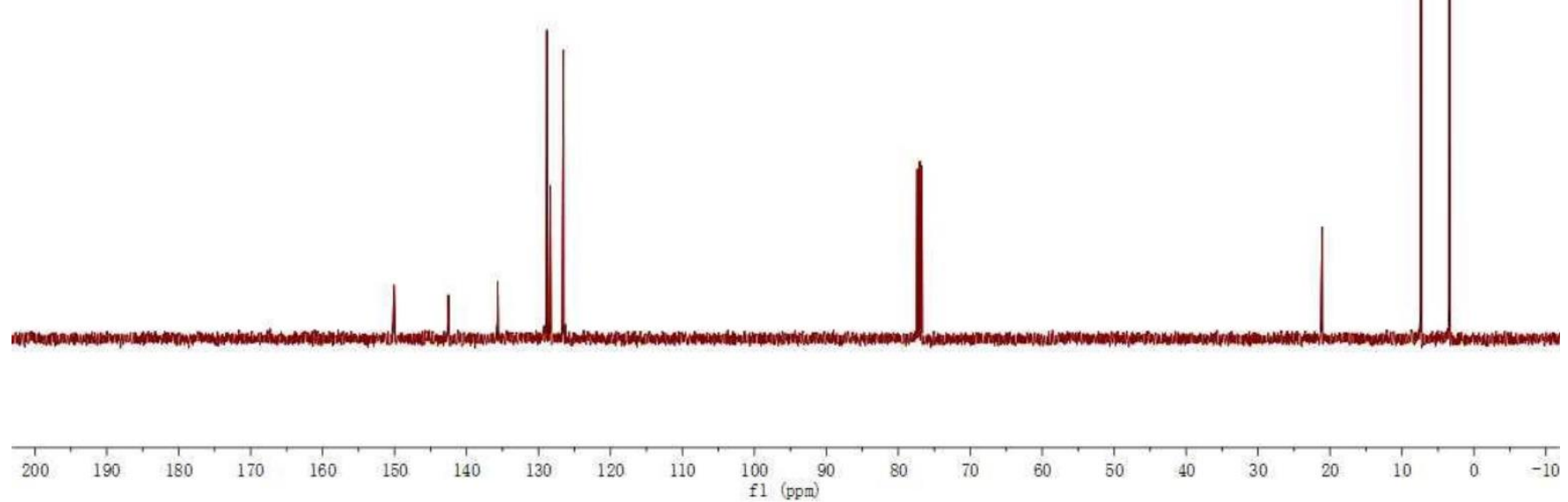

Figure S69. ${ }^{13} \mathrm{C}$ NMR spectrum of $\mathbf{P 2 4}$. 


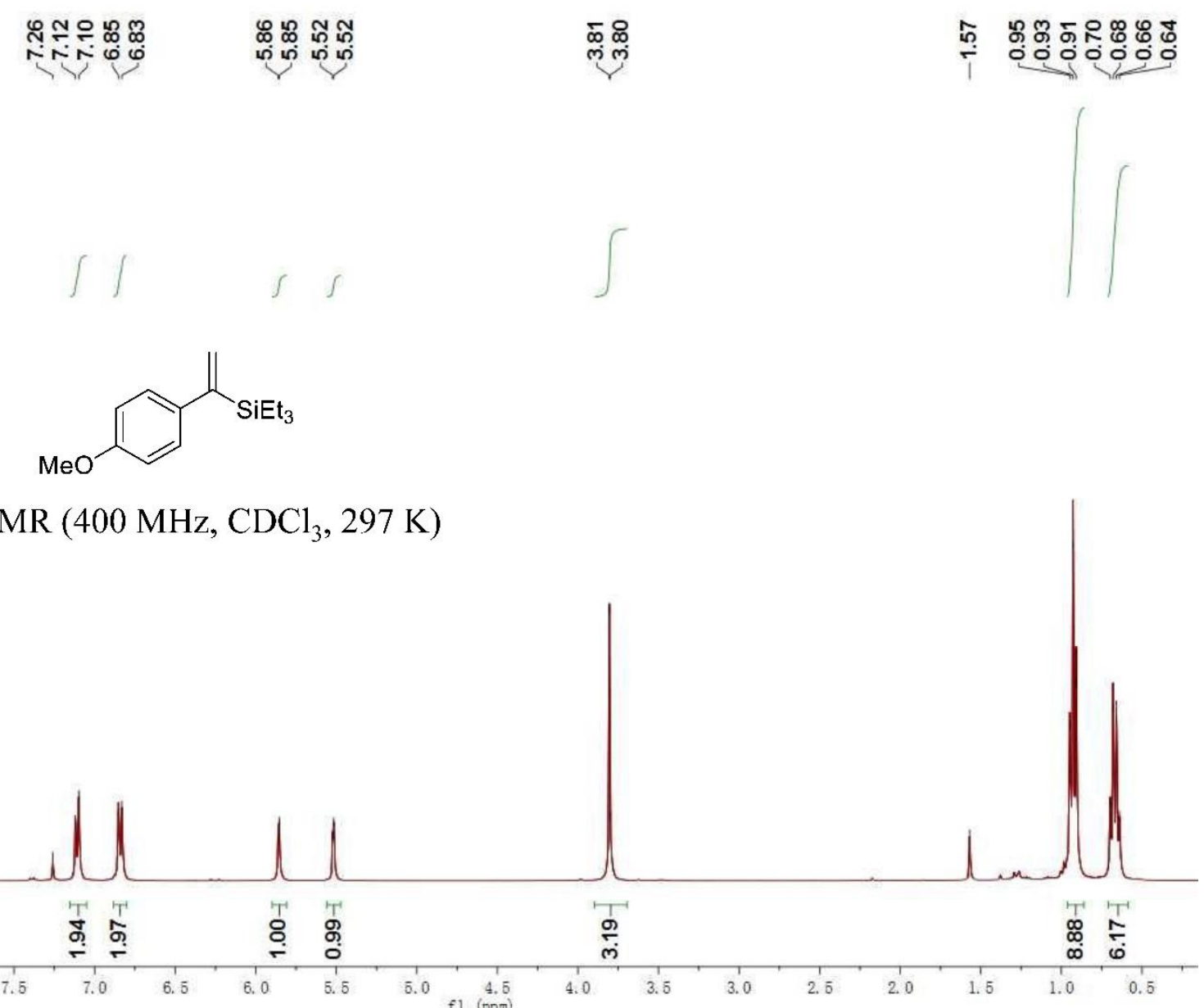

Figure S70. ${ }^{1} \mathrm{H}$ NMR spectrum of $\mathbf{P 2 5}$.

S97 


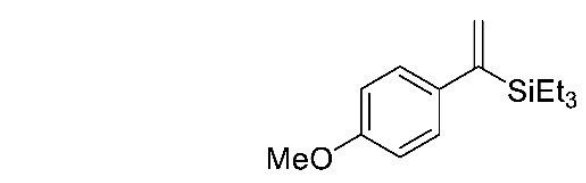

${ }^{13} \mathrm{C}$ NMR (100 MHz, $\left.\mathrm{CDCl}_{3}, 297 \mathrm{~K}\right)$

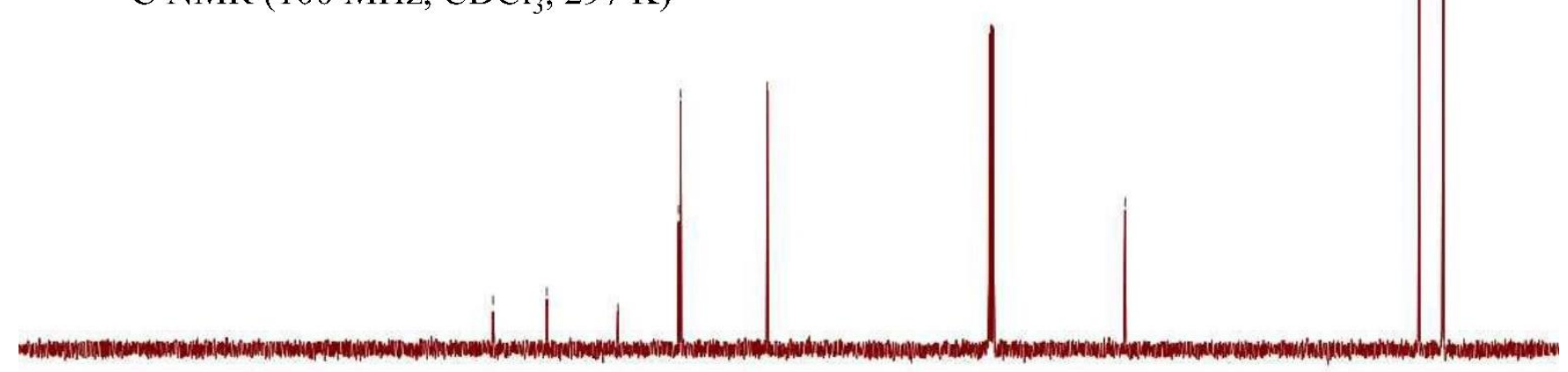

$\begin{array}{llllllllll}{ }_{230}^{\prime} & 220 & 210 & 200 & 190 & 180 & 170 & 160 & 150\end{array}$ $150 \quad 140$ $120 \underset{\mathrm{f} 1}{110} 1 \mathrm{ppm})$

Figure S71. ${ }^{13} \mathrm{C}$ NMR spectrum of $\mathbf{P 2 5}$. 


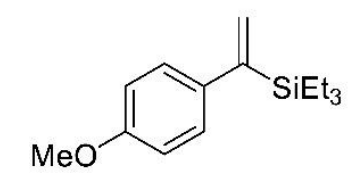

${ }^{29} \mathrm{Si} \mathrm{NMR}\left(79 \mathrm{MHz}, \mathrm{CDCl}_{3}, 297 \mathrm{~K}\right)$

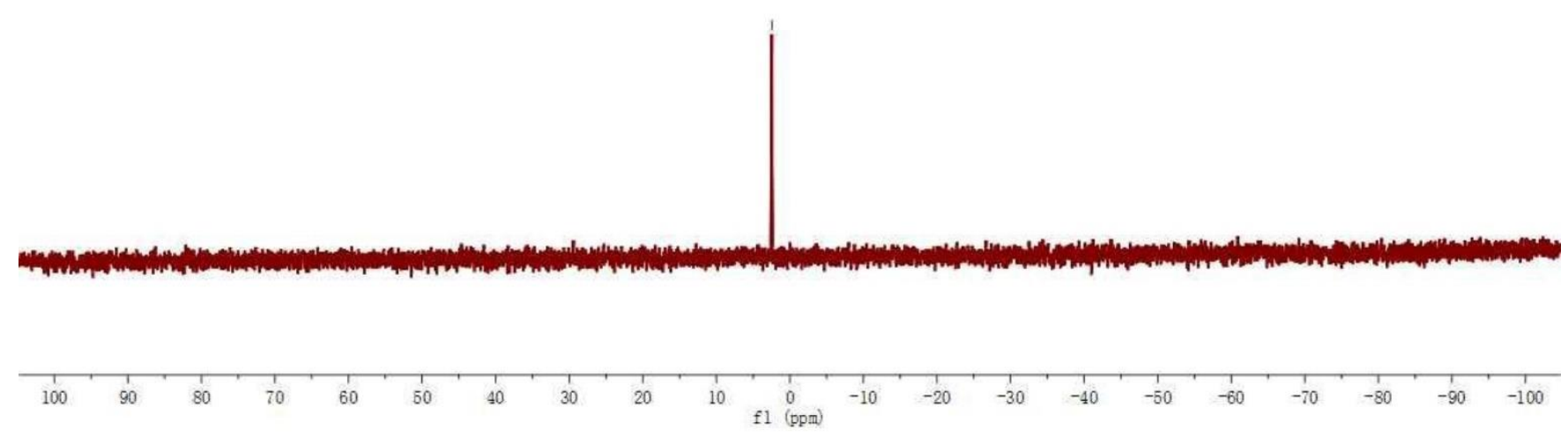

Figure S72. ${ }^{29} \mathrm{Si}$ NMR spectrum of $\mathbf{P 2 5}$.

599 


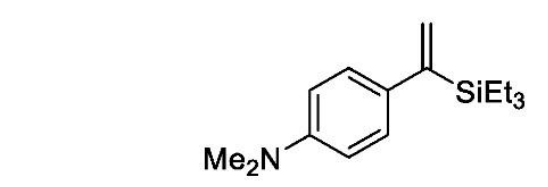

${ }^{1} \mathrm{H} \mathrm{NMR}\left(\mathrm{CDCl}_{3}, 400 \mathrm{MHz}, 298 \mathrm{~K}\right)$

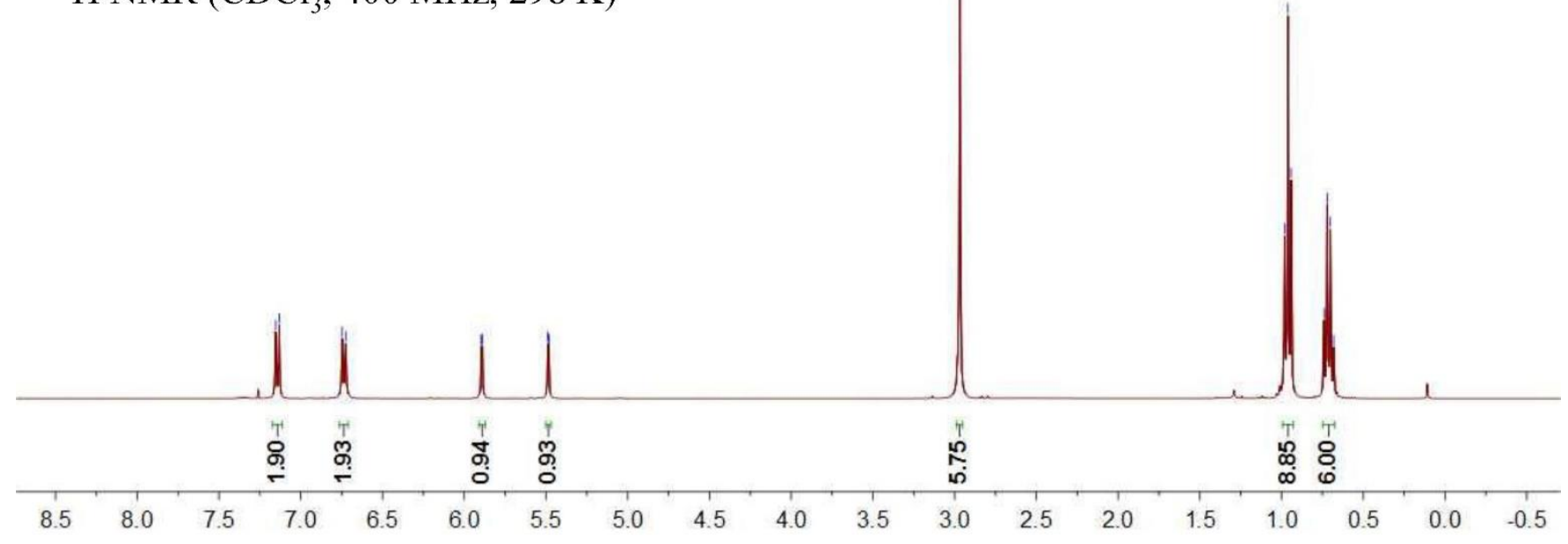

Figure S73. ${ }^{1} \mathrm{H}$ NMR spectrum of $\mathbf{P 2 6}$. 


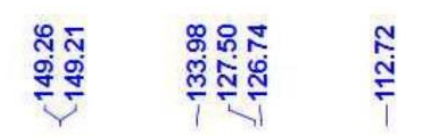

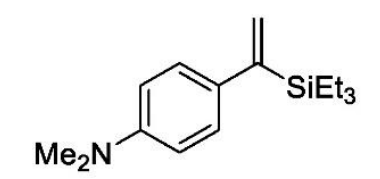

${ }^{13} \mathrm{C} \mathrm{NMR}\left(\mathrm{CDCl}_{3}, 100 \mathrm{MHz}, 298 \mathrm{~K}\right)$
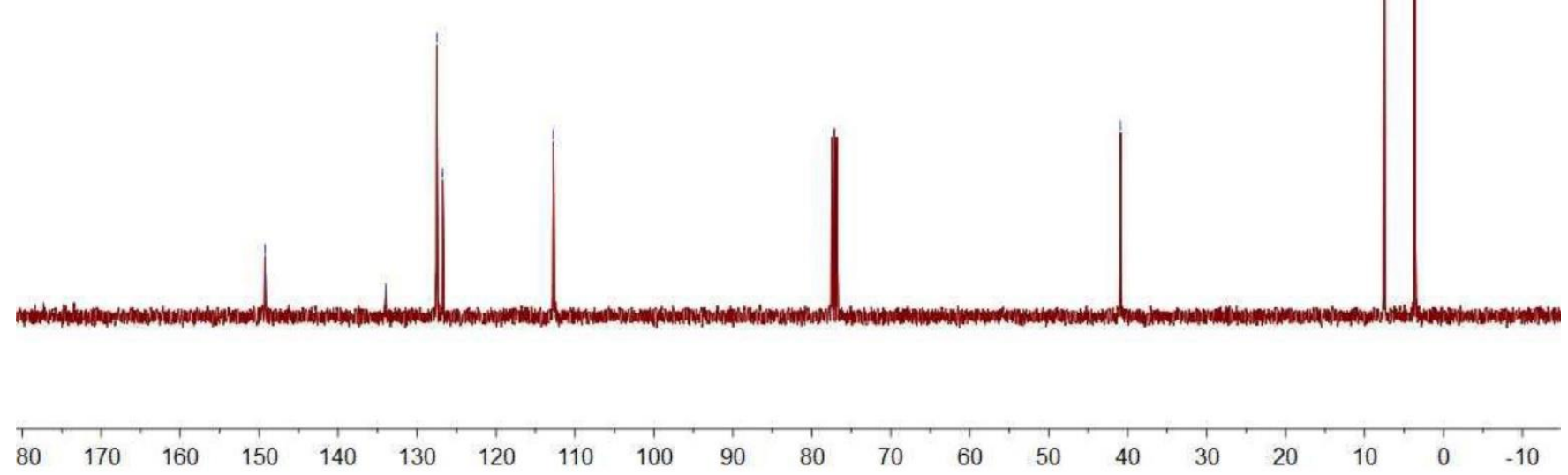

Figure S74. ${ }^{13} \mathrm{C}$ NMR spectrum of $\mathbf{P 2 6}$. 


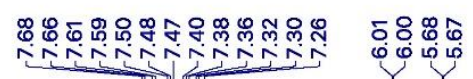

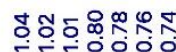

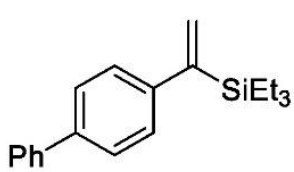

IIII

${ }^{1} \mathrm{H} \mathrm{NMR}\left(\mathrm{CDCl}_{3}, 400 \mathrm{MHz}, 298 \mathrm{~K}\right)$

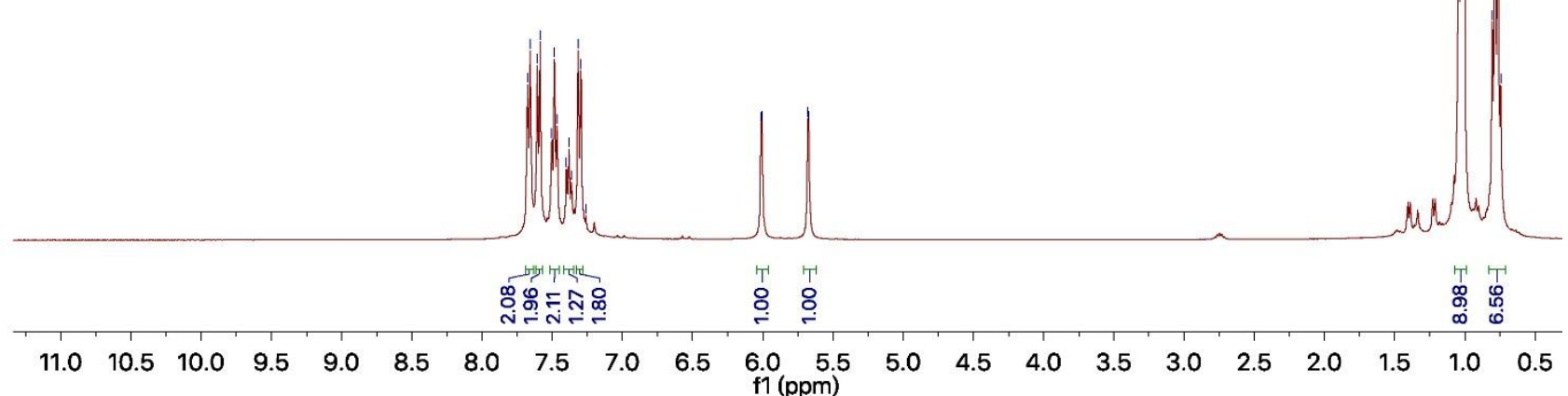

Figure S75. ${ }^{1} \mathrm{H}$ NMR spectrum of $\mathbf{P 2 7}$. 


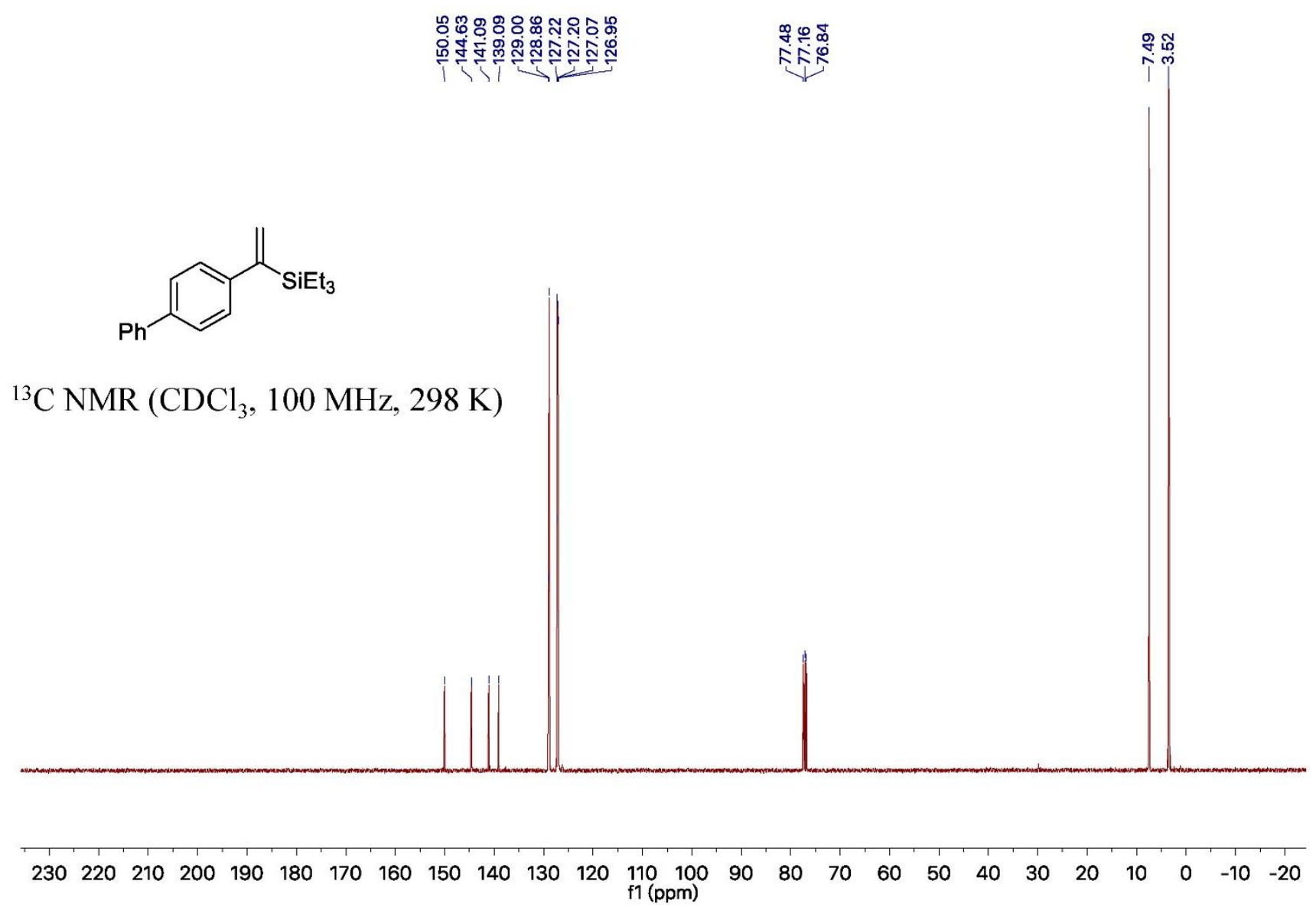

Figure S76. ${ }^{13} \mathrm{C}$ NMR spectrum of $\mathbf{P 2 7}$. 


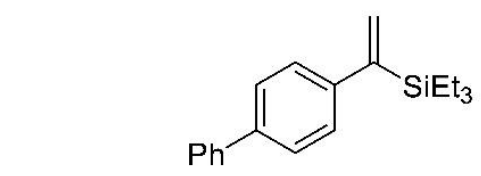

${ }^{29} \mathrm{Si} \mathrm{NMR}\left(79 \mathrm{MHz}, \mathrm{CDCl}_{3}, 297 \mathrm{~K}\right)$

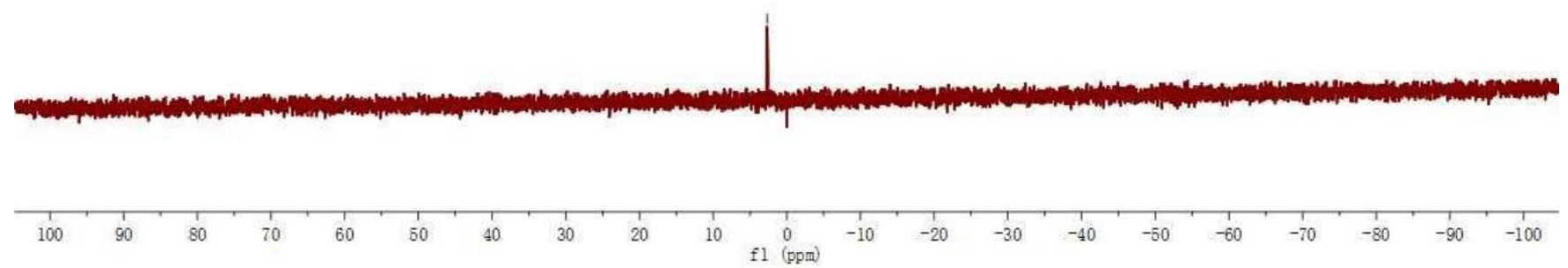

Figure S77. ${ }^{29} \mathrm{Si}$ NMR spectrum of $\mathbf{P 2 7}$. 


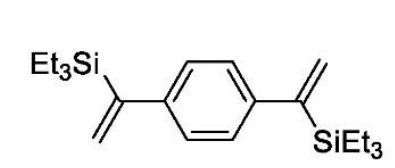

${ }^{1} \mathrm{H} \mathrm{NMR}\left(\mathrm{CDCl}_{3}, 400 \mathrm{MHz}, 292 \mathrm{~K}\right)$

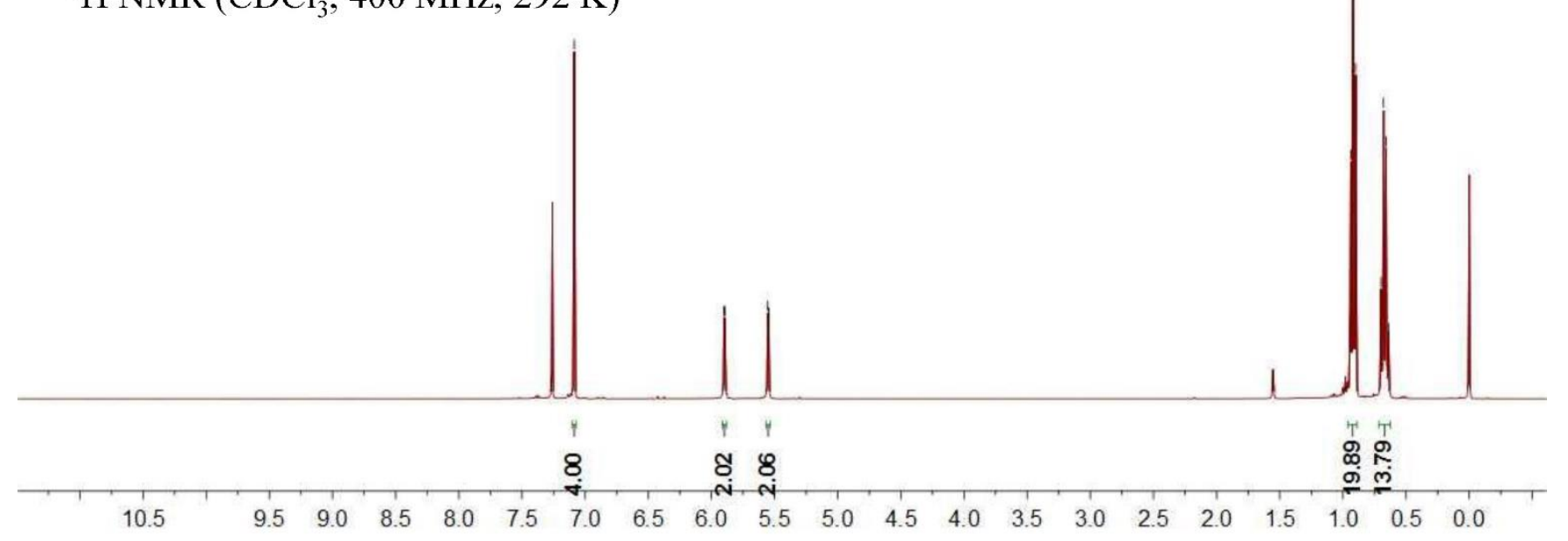

Figure S78. ${ }^{1} \mathrm{H}$ NMR spectrum of $\mathbf{P 2 8}$. 


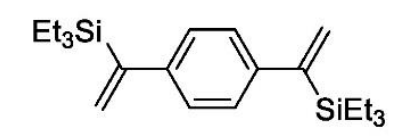

${ }^{13} \mathrm{C} \mathrm{NMR}\left(\mathrm{CDCl}_{3}, 100 \mathrm{MHz}, 298 \mathrm{~K}\right)$

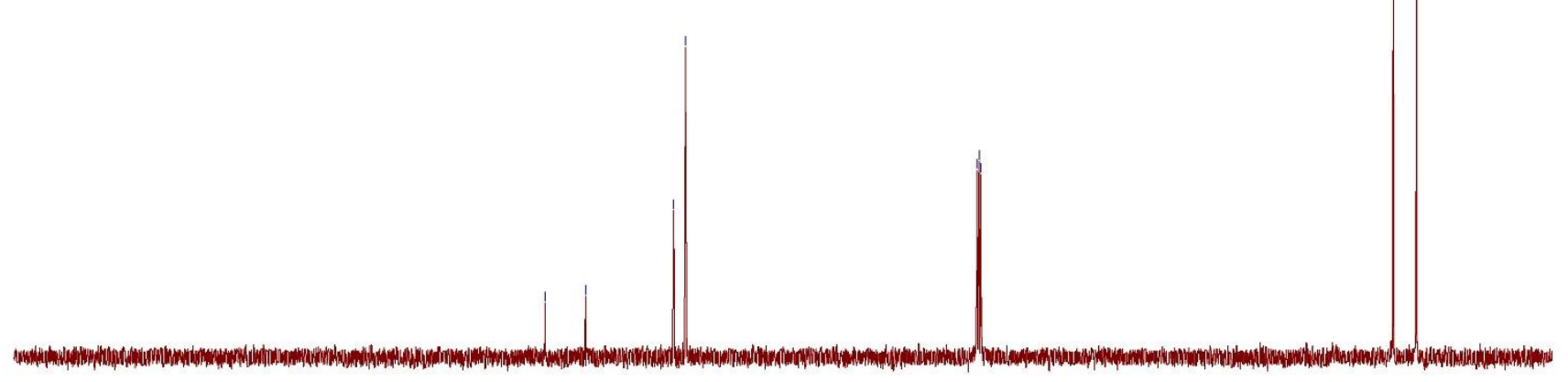

$\begin{array}{llllllllllllllllllllllllllll}230 & 220 & 210 & 200 & 190 & 180 & 170 & 160 & 150 & 140 & 130 & 120 & 110 & 100 & 90 & 80 & 70 & 60 & 50 & 40 & 30 & 20 & 10 & 0 & -10\end{array}$

Figure S79. ${ }^{13} \mathrm{C}$ NMR spectrum of $\mathbf{P 2 8}$. 


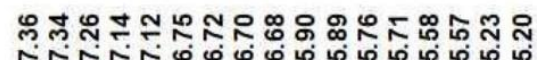

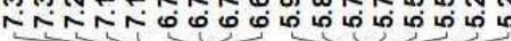

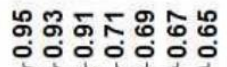

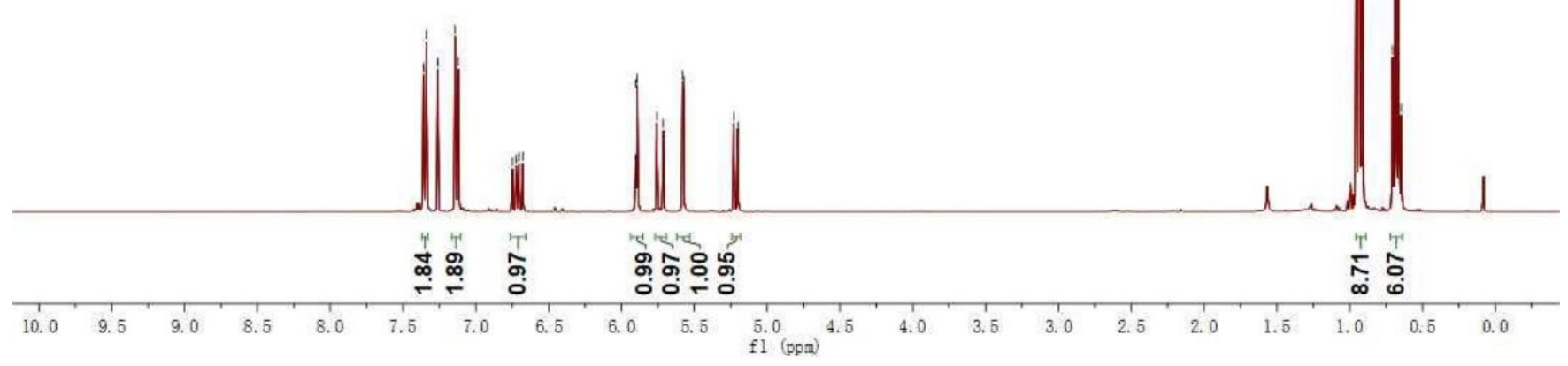

Figure S80. ${ }^{1} \mathrm{H}$ NMR spectrum of $\mathbf{P 2 9}$. 

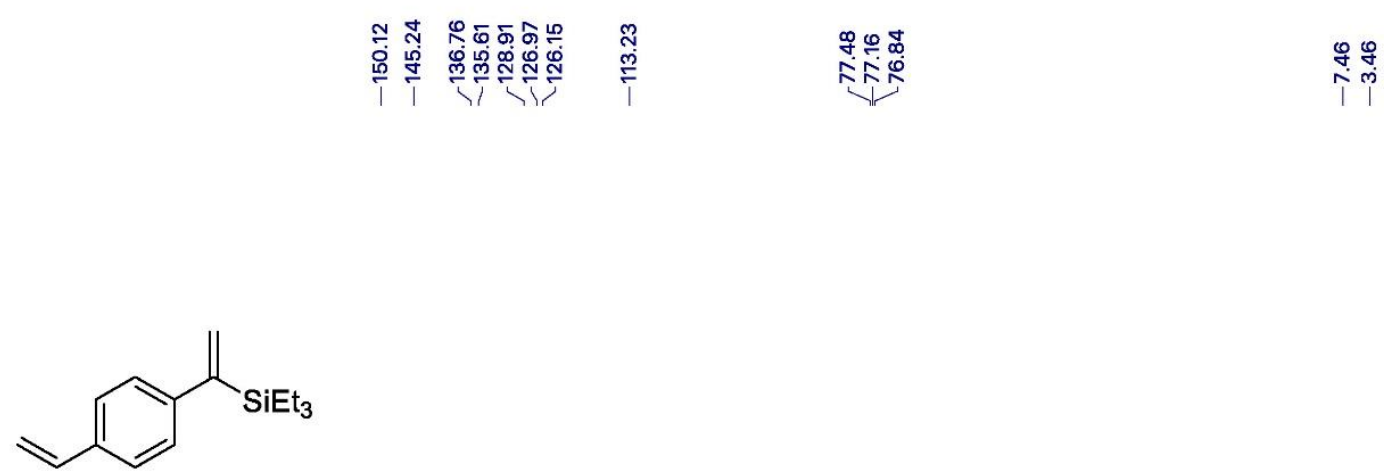

${ }^{13} \mathrm{C} \mathrm{NMR}\left(\mathrm{CDCl}_{3}, 100 \mathrm{MHz}, 298 \mathrm{~K}\right)$

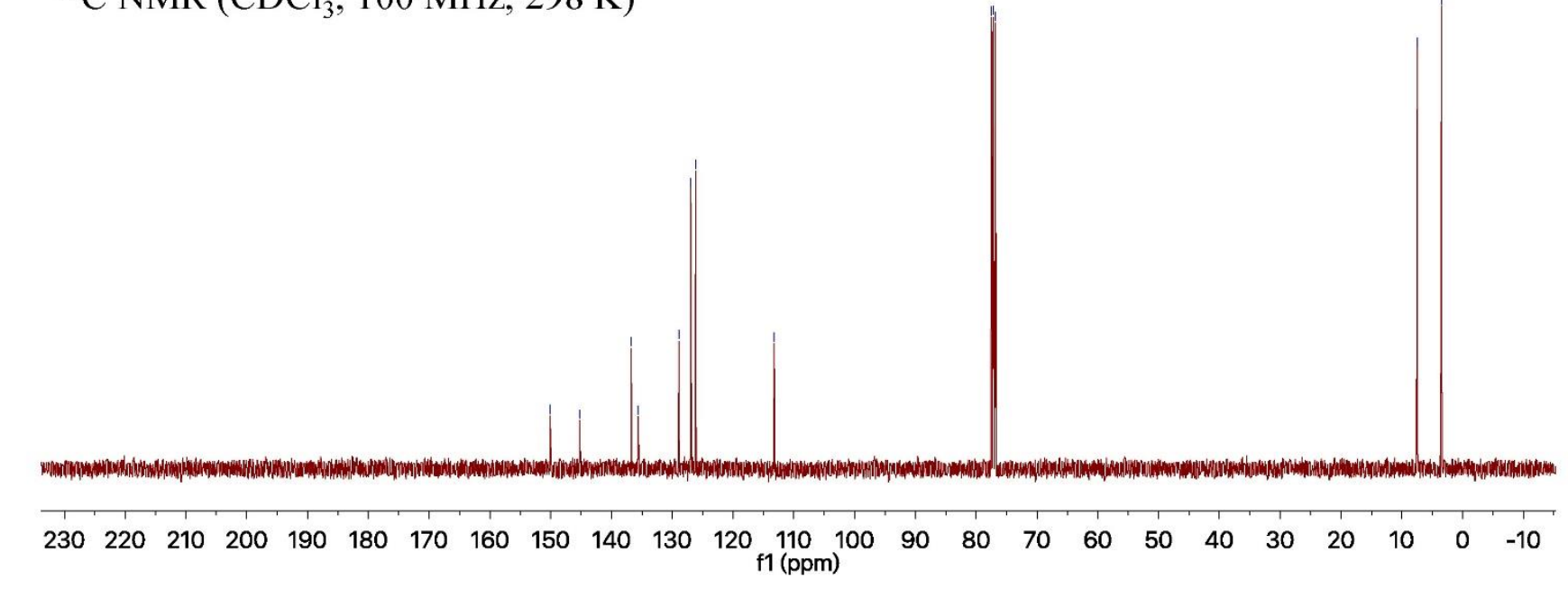

Figure S81. ${ }^{13} \mathrm{C}$ NMR spectrum of $\mathbf{P 2 9}$. 


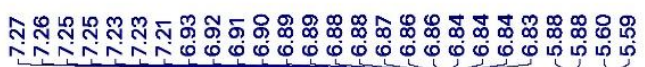

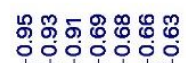
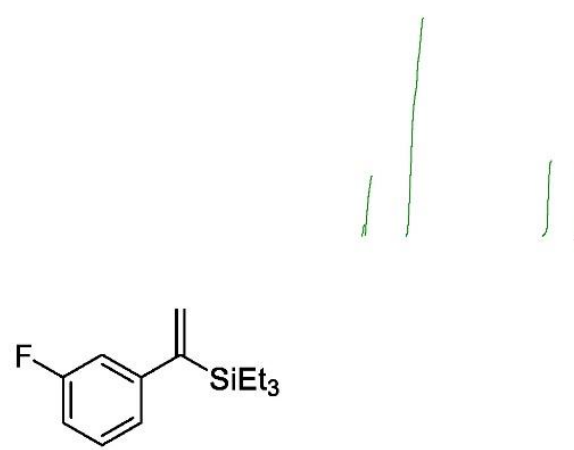

${ }^{1} \mathrm{H} \mathrm{NMR}\left(\mathrm{CDCl}_{3}, 400 \mathrm{MHz}, 296 \mathrm{~K}\right)$

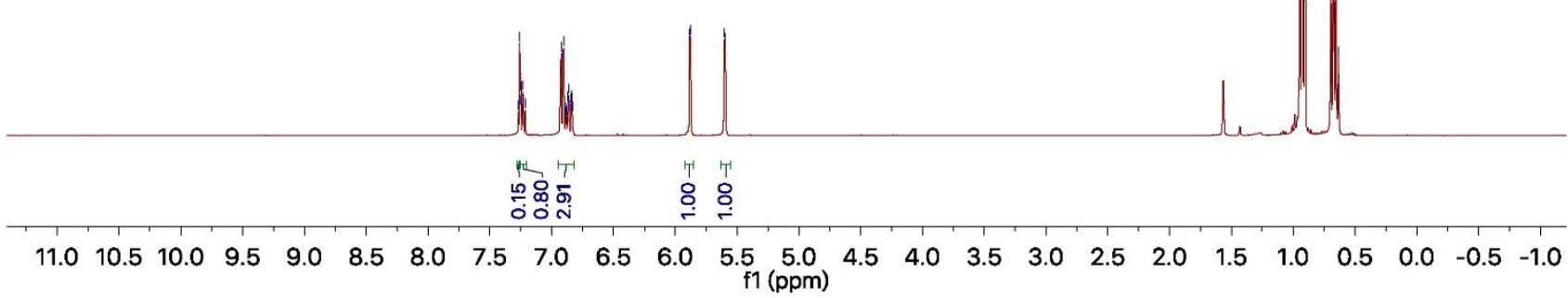

Figure S82. ${ }^{1} \mathrm{H}$ NMR spectrum of $\mathbf{P 3 0}$. 


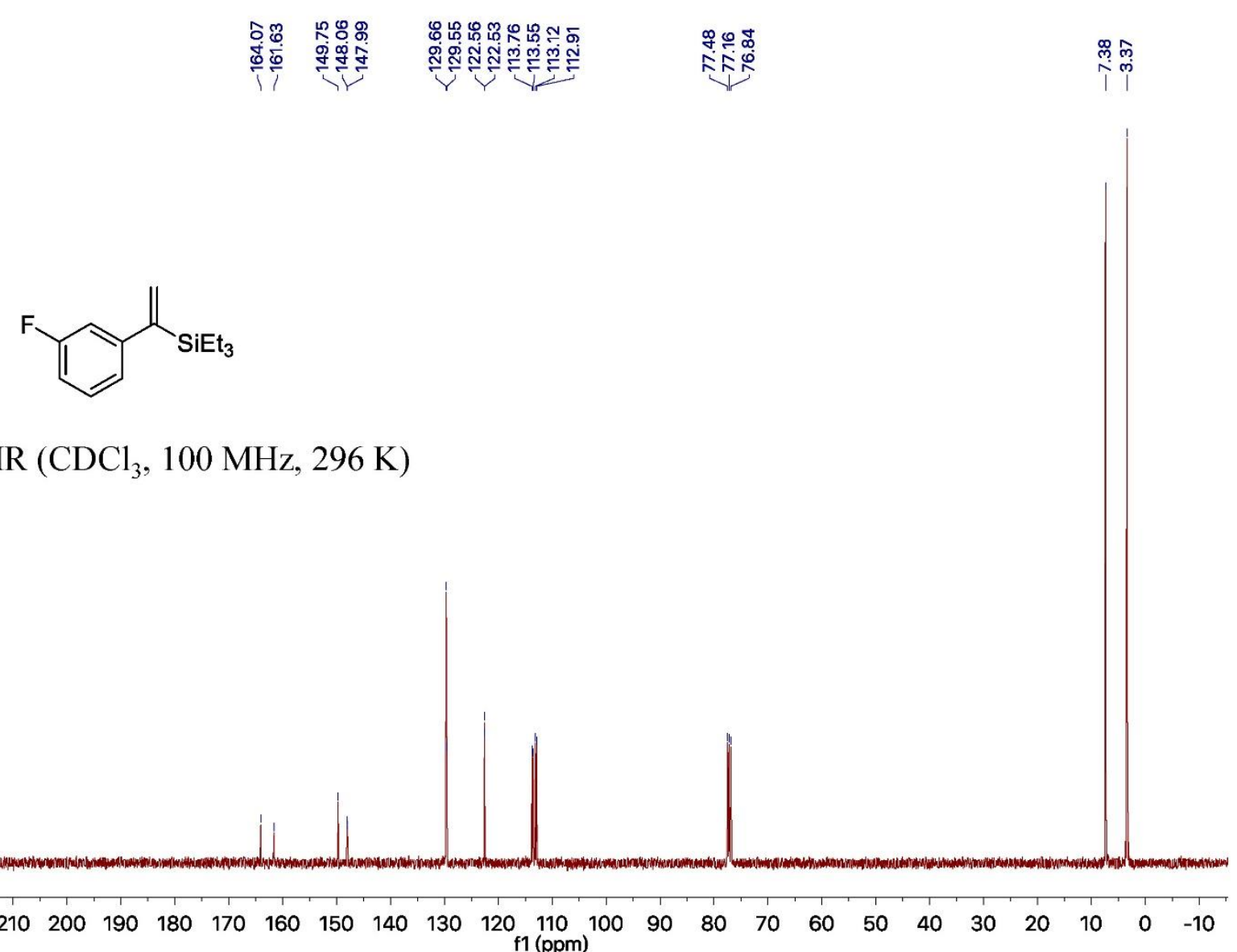

Figure S83. ${ }^{13} \mathrm{C}$ NMR spectrum of $\mathbf{P 3 0}$. 


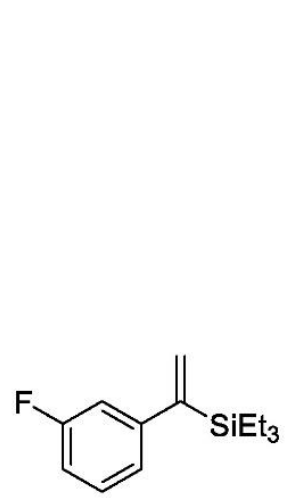

${ }^{19} \mathrm{~F}$ NMR $\left(\mathrm{CDCl}_{3}, 376 \mathrm{MHz}, 296 \mathrm{~K}\right)$

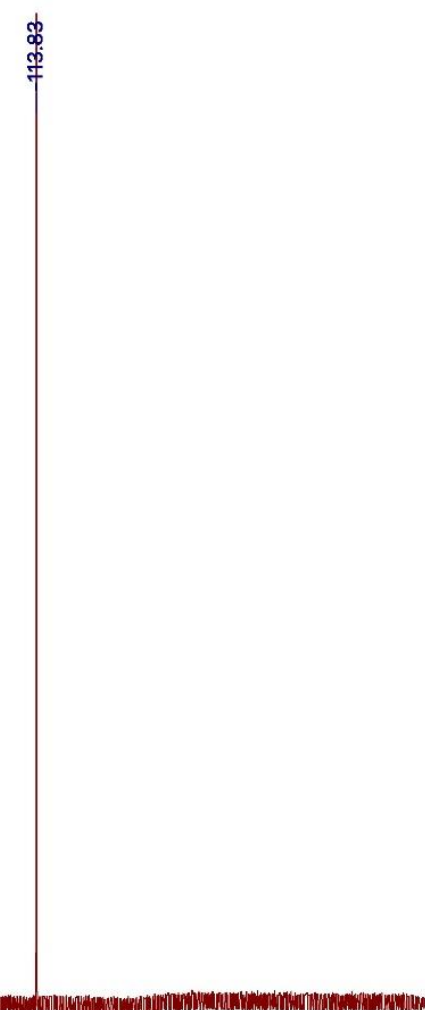

m.1.

Figure S84. ${ }^{19} \mathrm{~F}$ NMR spectrum of P30. 


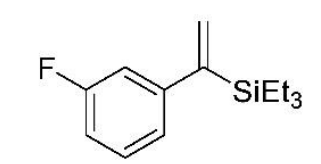

${ }^{29} \mathrm{Si} \mathrm{NMR}\left(79 \mathrm{MHz}, \mathrm{CDCl}_{3}, 297 \mathrm{~K}\right)$

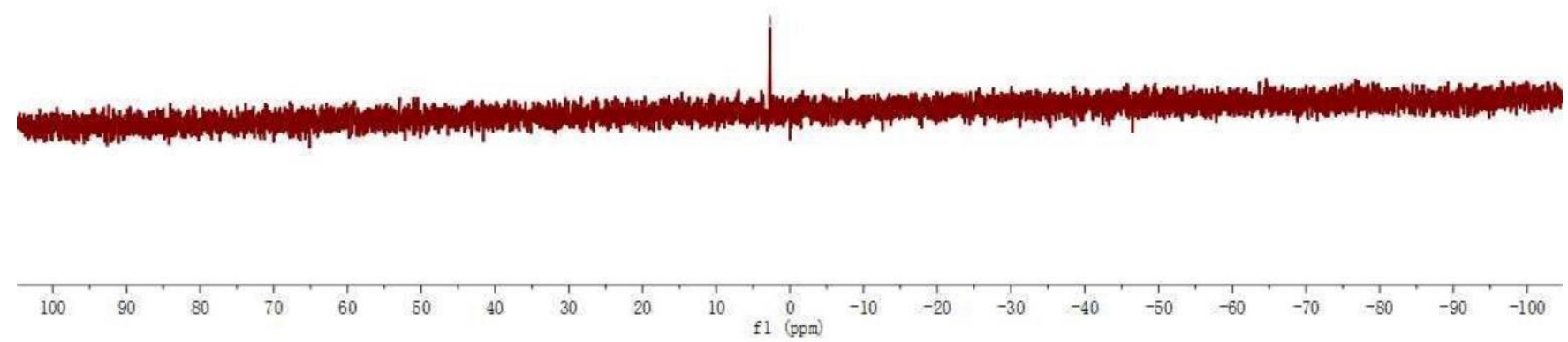

Figure S85. ${ }^{29} \mathrm{Si}$ NMR spectrum of P30. 


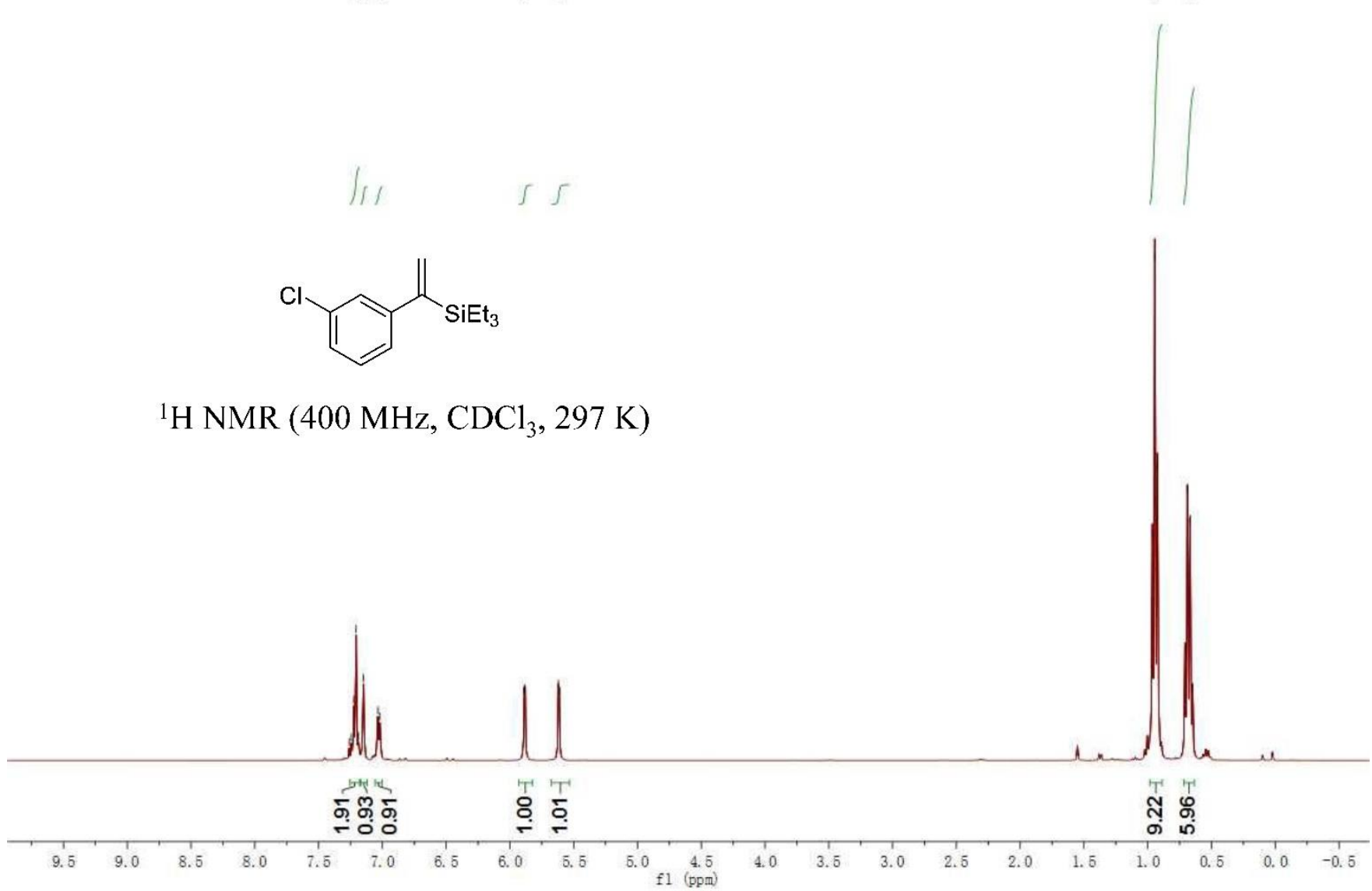

${ }^{1} \mathrm{H}$ NMR (400 MHz, CDCl $\left.3,297 \mathrm{~K}\right)$

Figure S86. ${ }^{1} \mathrm{H}$ NMR spectrum of $\mathbf{P 3 1}$. 


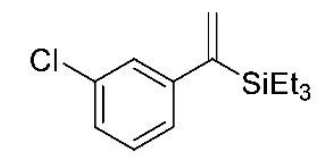

${ }^{13} \mathrm{C}$ NMR $\left(100 \mathrm{MHz}, \mathrm{CDCl}_{3}, 297 \mathrm{~K}\right)$
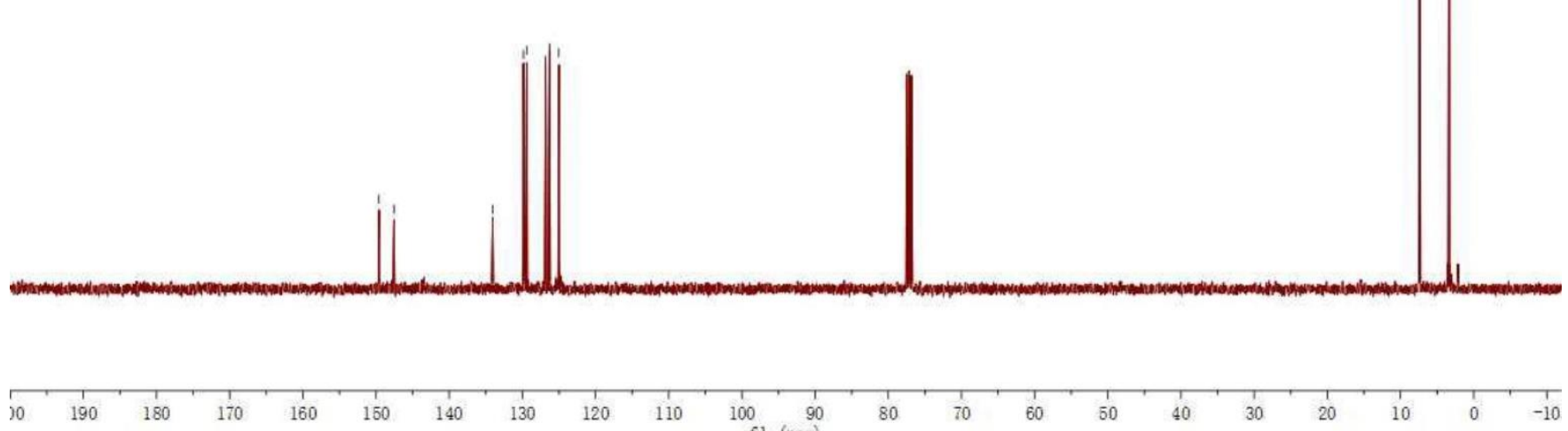

Figure S87. ${ }^{13} \mathrm{C}$ NMR spectrum of $\mathbf{P 3 1}$. 


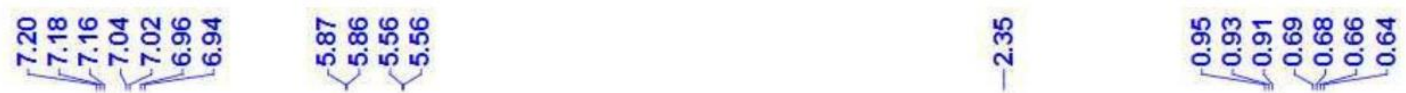

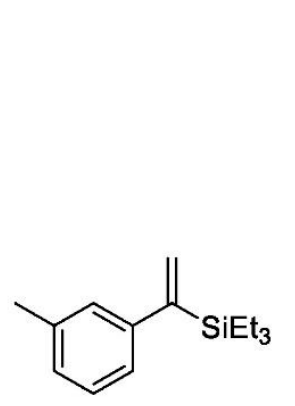

${ }^{1} \mathrm{H}$ NMR (CDCl, 400 MHz, $\left.296 \mathrm{~K}\right)$

Figure S88. ${ }^{1} \mathrm{H}$ NMR spectrum of $\mathbf{P 3 2}$. 

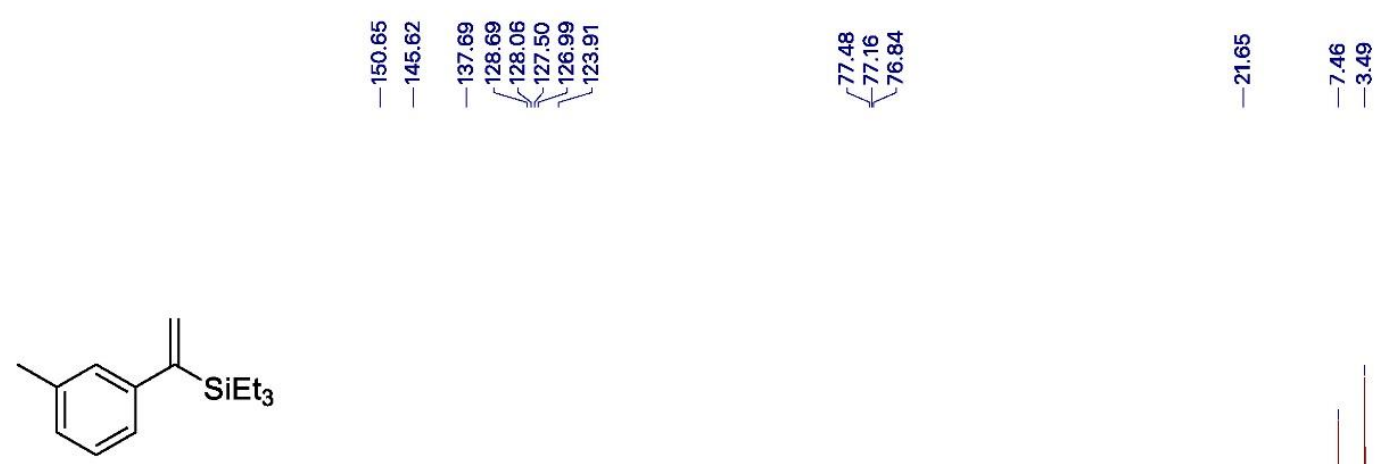

${ }^{13} \mathrm{C} \mathrm{NMR}\left(\mathrm{CDCl}_{3}, 100 \mathrm{MHz}, 296 \mathrm{~K}\right)$

$\begin{array}{llllllllllllllllllllllll}220 & 210 & 200 & 190 & 180 & 170 & 160 & 150 & 140 & 130 & 120 & 110 & 100 & 90 & 80 & 70 & 60 & 50 & 40 & 30 & 20 & 10 & 0 & -10\end{array}$

Figure S89. ${ }^{13} \mathrm{C}$ NMR spectrum of $\mathbf{P 3 2}$. 


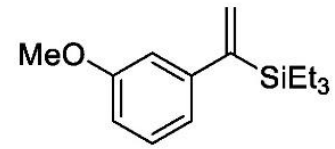

'H NMR (CDCl, 400 MHz, $303 \mathrm{~K})$

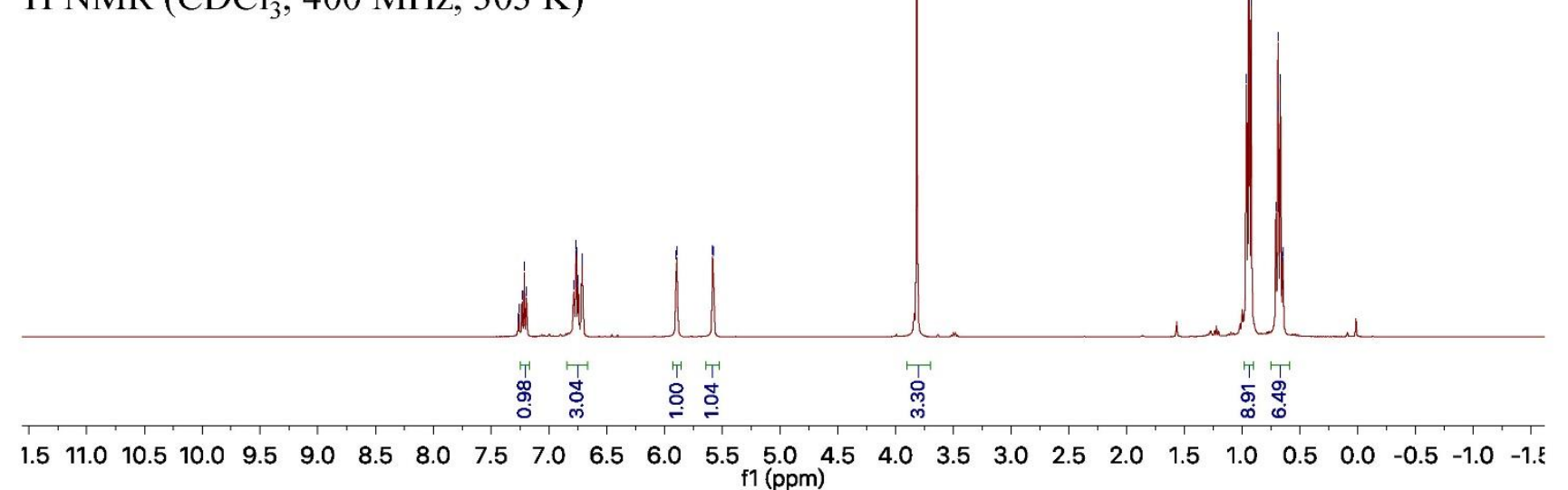

Figure S90. ${ }^{1} \mathrm{H}$ NMR spectrum of $\mathbf{P 3 3}$. 

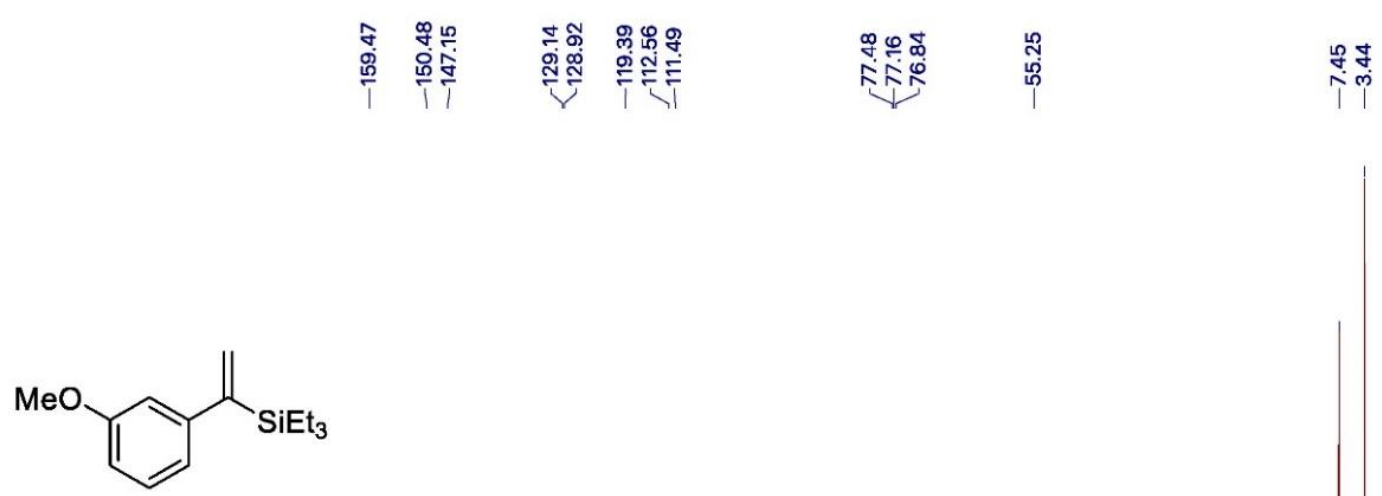

${ }^{13} \mathrm{C} \mathrm{NMR}\left(\mathrm{CDCl}_{3}, 100 \mathrm{MHz}, 303 \mathrm{~K}\right)$

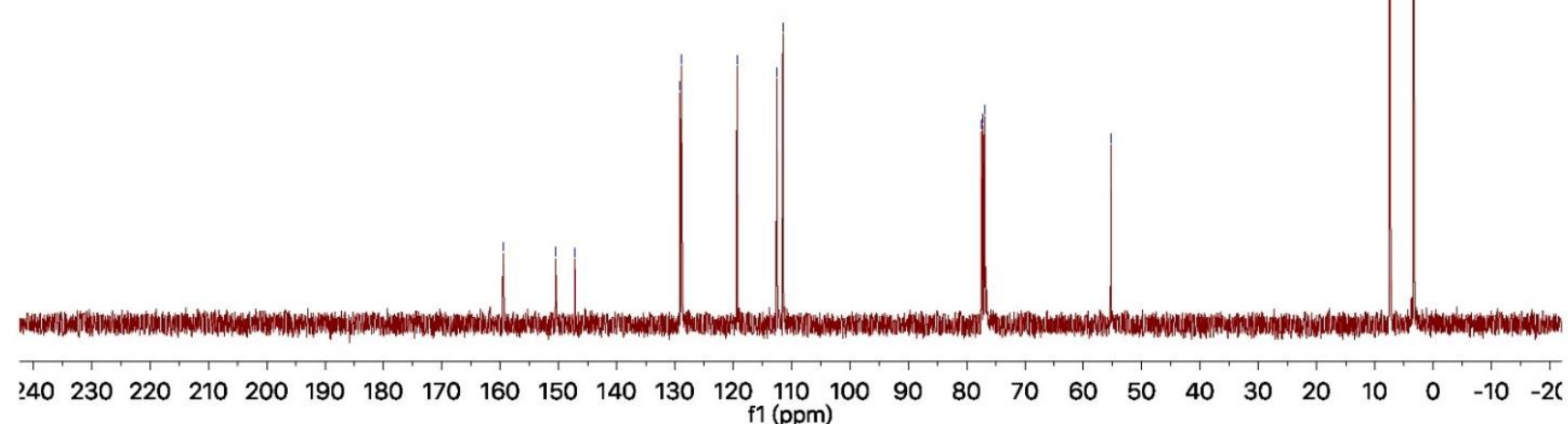

Figure S91. ${ }^{13} \mathrm{C}$ NMR spectrum of $\mathbf{P 3 3}$. 


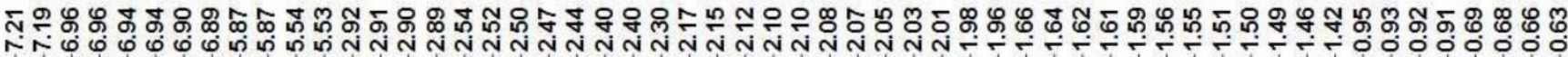

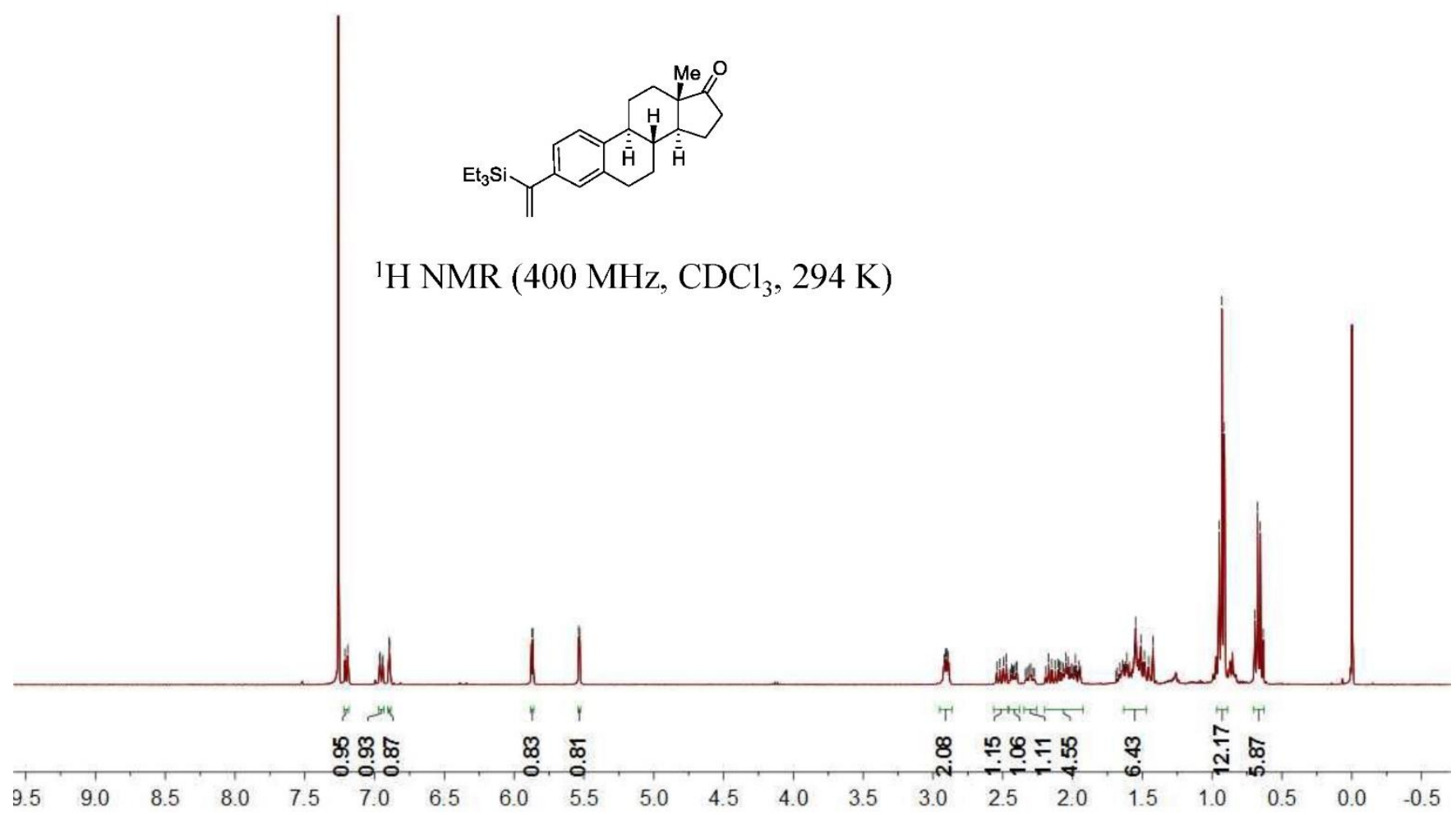

Figure S92. ${ }^{1} \mathrm{H}$ NMR spectrum of P34. 


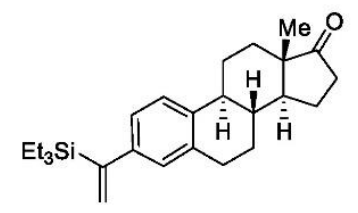

${ }^{13} \mathrm{C}$ NMR $\left(100 \mathrm{MHz}, \mathrm{CDCl}_{3}, 294 \mathrm{~K}\right)$

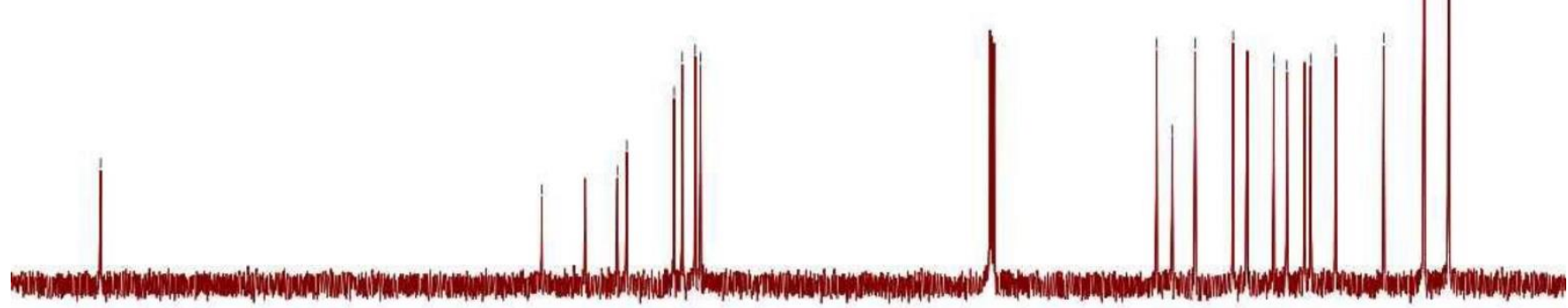

Figure S93. ${ }^{13} \mathrm{C}$ NMR spectrum of $\mathbf{P 3 4}$. 

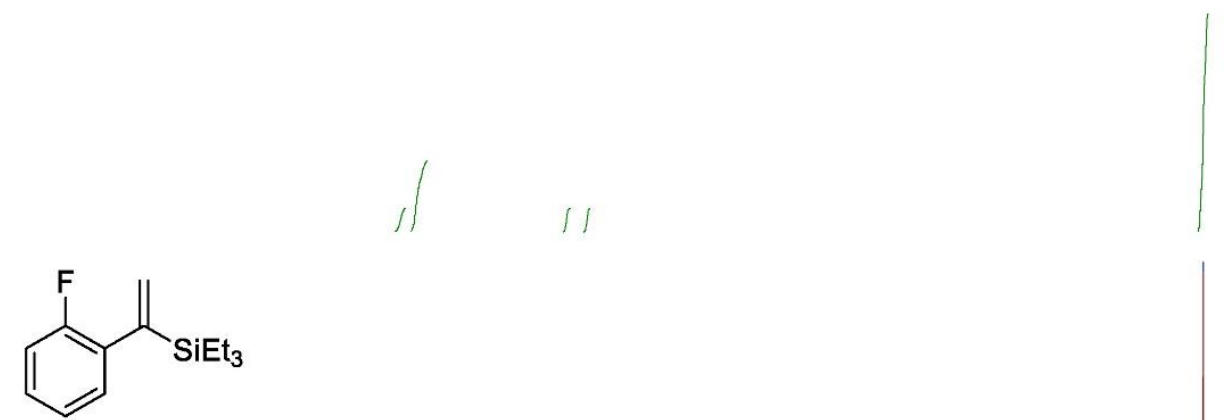

'H NMR (CDCl 3,400 MHz, 298 K)

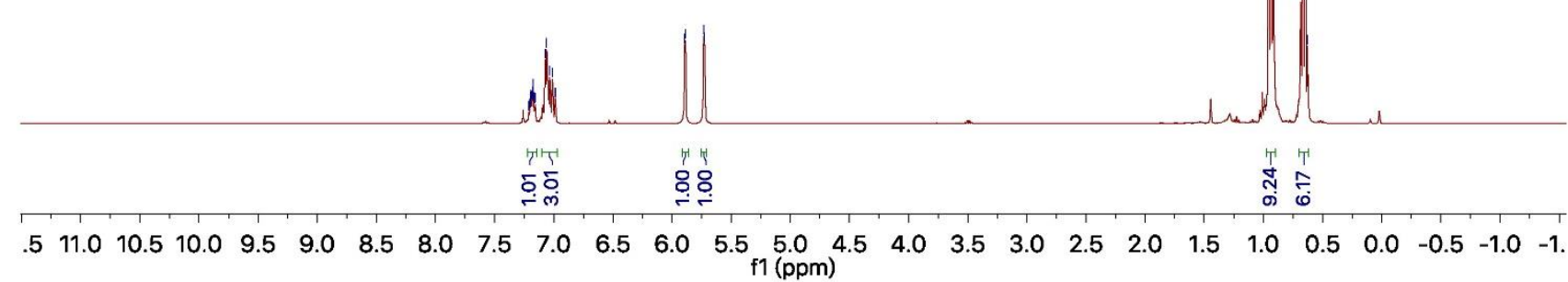

Figure S94. ${ }^{1} \mathrm{H}$ NMR spectrum of $\mathbf{P 3 5}$. 


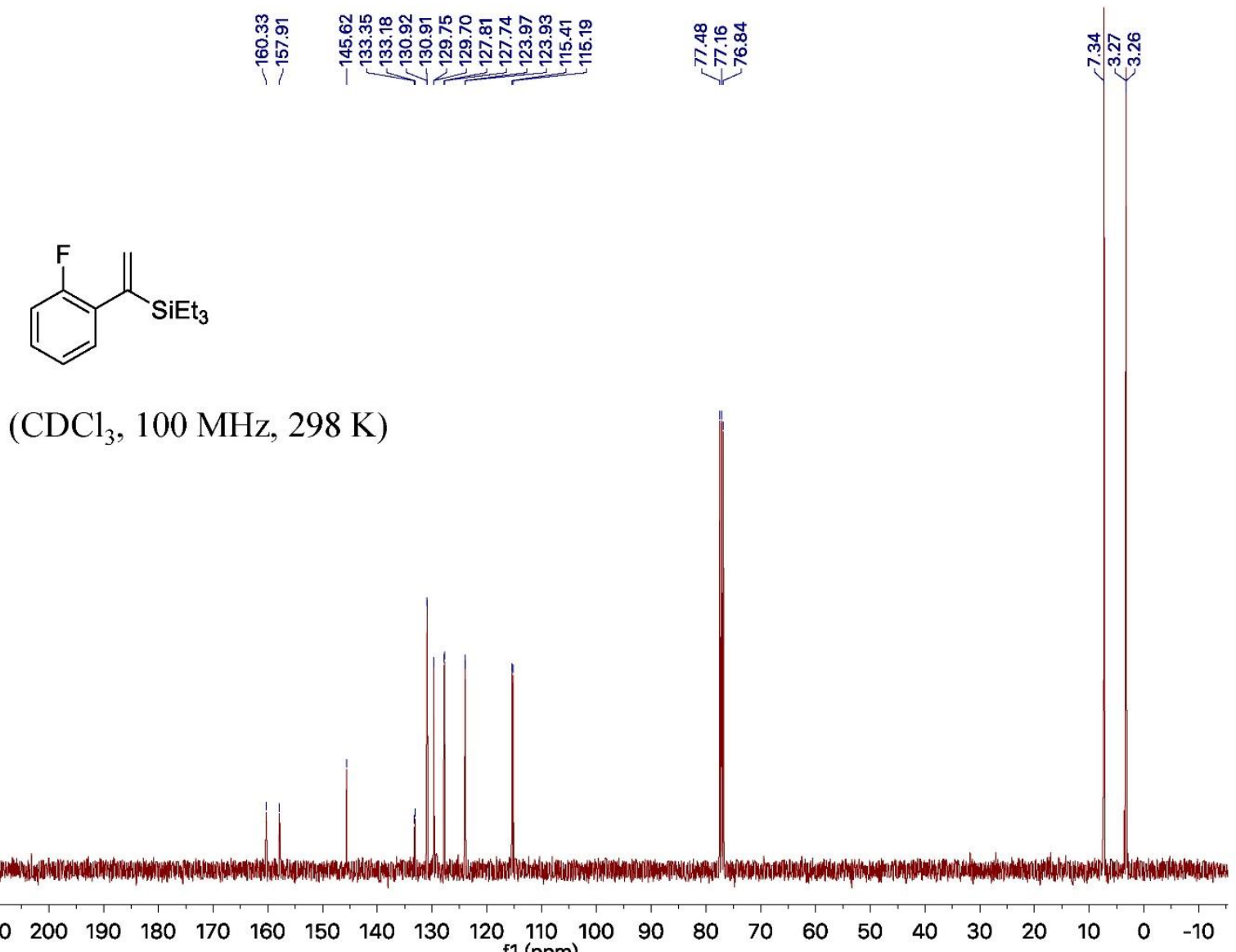

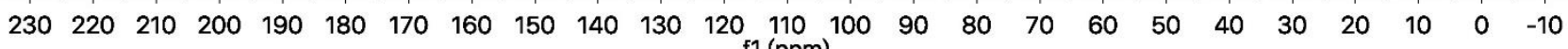

Figure S95. ${ }^{13} \mathrm{C}$ NMR spectrum of $\mathbf{P 3 5}$. 


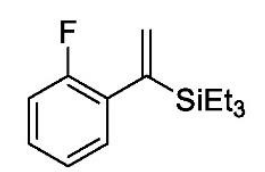

${ }^{19} \mathrm{~F} \mathrm{NMR}\left(\mathrm{CDCl}_{3}, 376 \mathrm{MHz}, 298 \mathrm{~K}\right)$

$\begin{array}{lllll}450 & 400 & 350 & 300 & 250\end{array}$

200

$\begin{array}{cc}150 & 100 \\ \mathrm{f} 1(\mathrm{ppm})\end{array}$

$50 \quad 0 \quad-50$

$-100-150$

$-200-250$

Figure S96. ${ }^{19} \mathrm{~F}$ NMR spectrum of $\mathbf{P 3 5}$. 


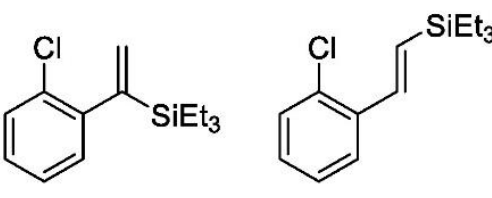

(4:1)

${ }^{1} \mathrm{H} \mathrm{NMR}\left(\mathrm{CDCl}_{3}, 400 \mathrm{MHz}, 298 \mathrm{~K}\right)$

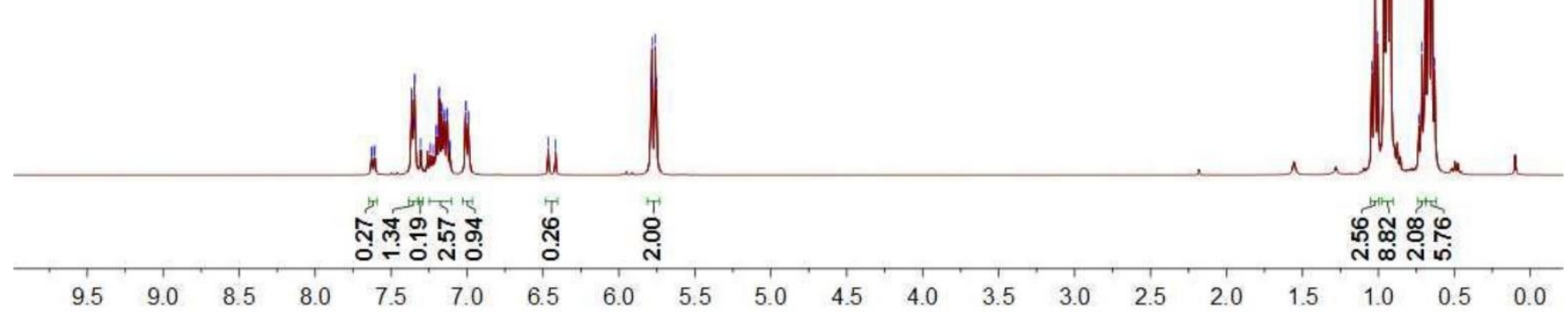

Figure S97. ${ }^{1} \mathrm{H}$ NMR spectrum of $\mathbf{P 3 6}$. 


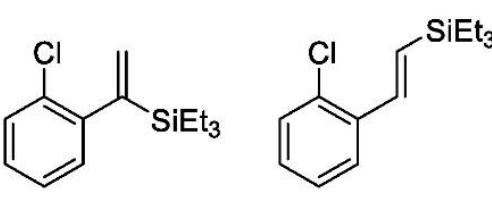

(4:1)

${ }^{13} \mathrm{C} \mathrm{NMR}\left(\mathrm{CDCl}_{3}, 100 \mathrm{MHz}, 294 \mathrm{~K}\right)$
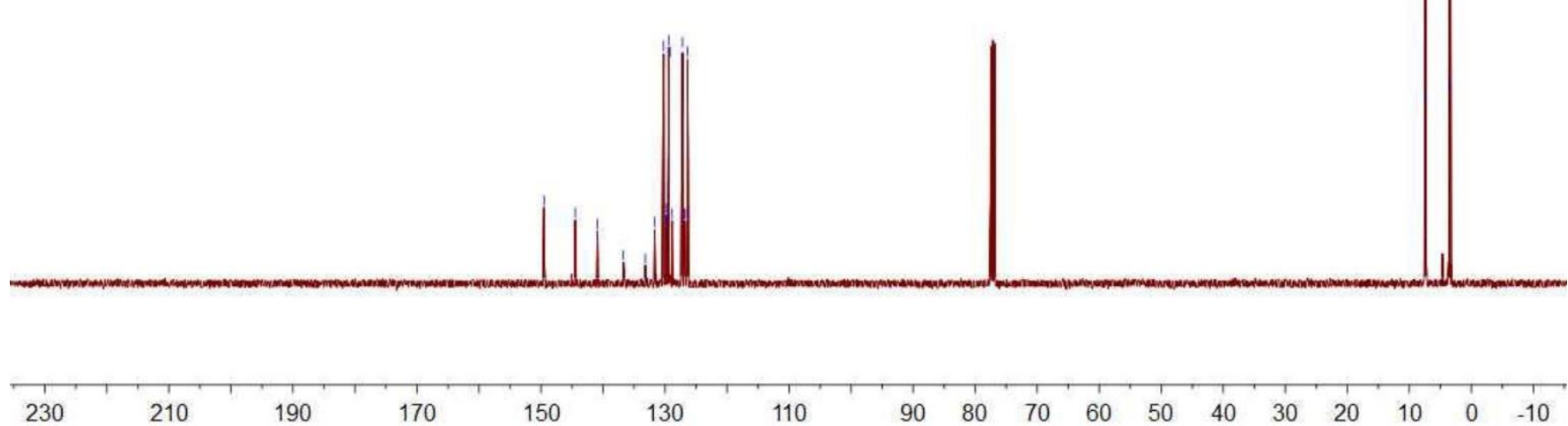

Figure S98. ${ }^{13} \mathrm{C}$ NMR spectrum of $\mathbf{P 3 6}$. 


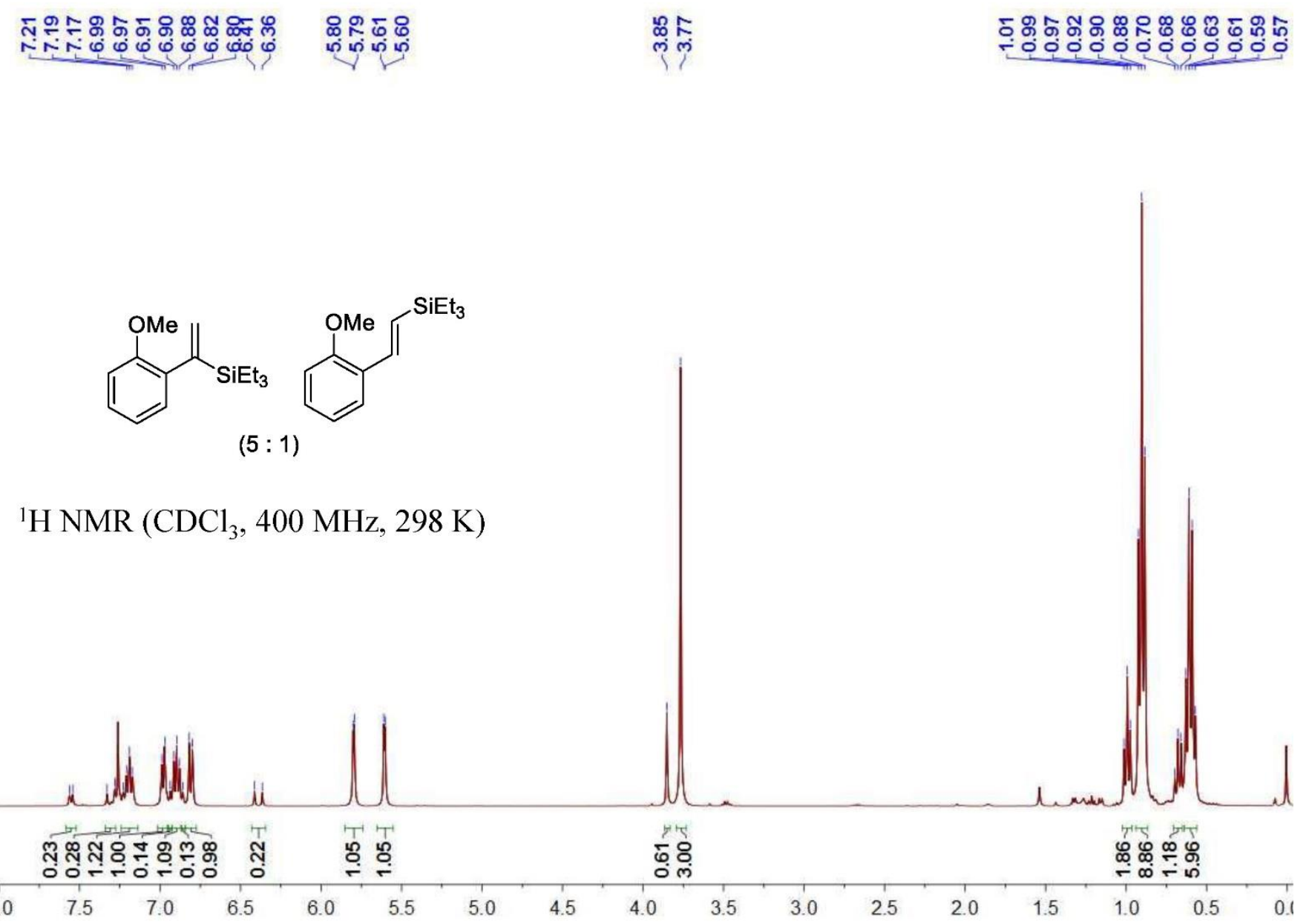

Figure S99. ${ }^{1} \mathrm{H}$ NMR spectrum of P37. 

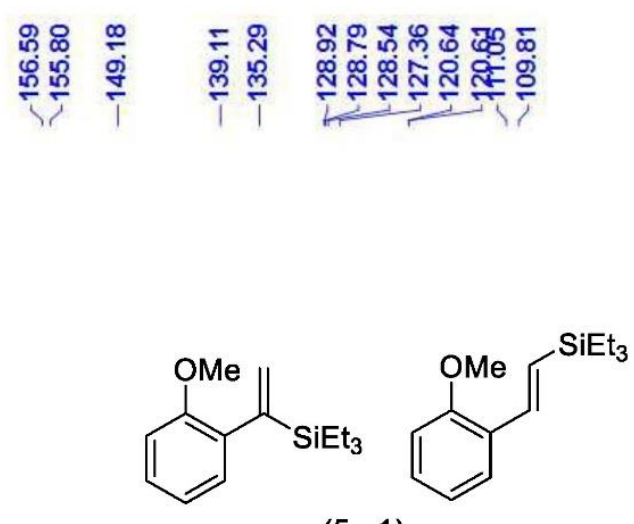

$(5: 1)$

${ }^{13} \mathrm{C} \mathrm{NMR}\left(\mathrm{CDCl}_{3}, 100 \mathrm{MHz}, 298 \mathrm{~K}\right)$

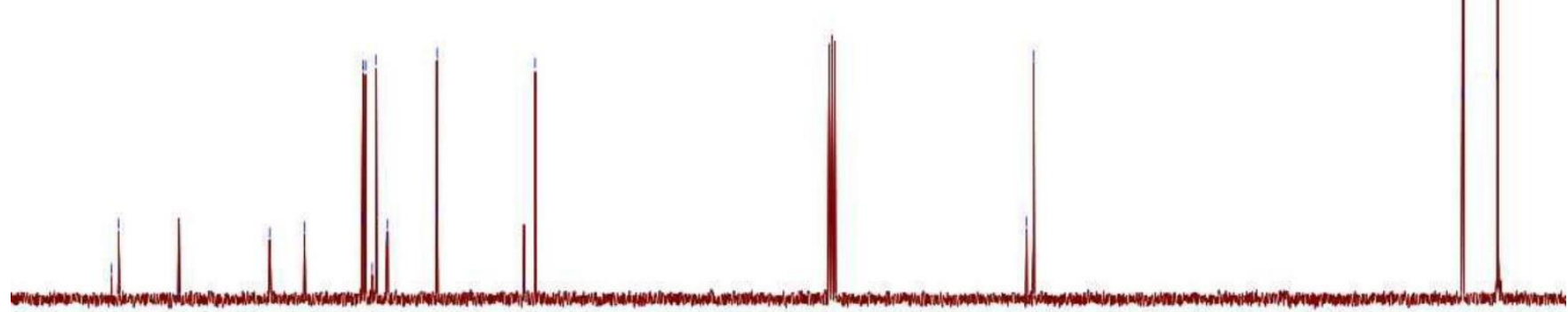

160

Figure S100. ${ }^{13} \mathrm{C}$ NMR spectrum of $\mathbf{P 3 7}$. 


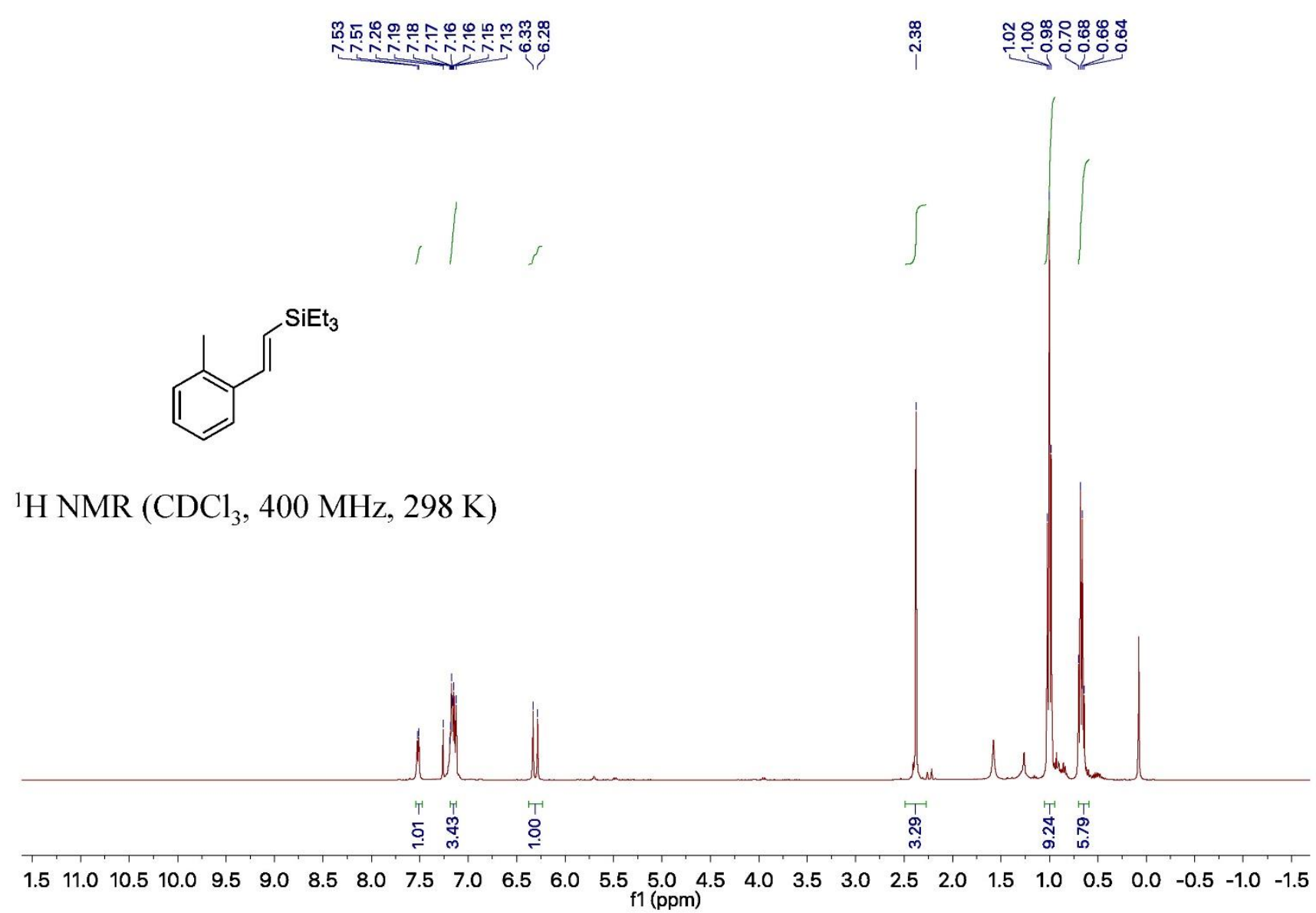

Figure S101. ${ }^{1} \mathrm{H}$ NMR spectrum of $\mathbf{P 3 8}$. 

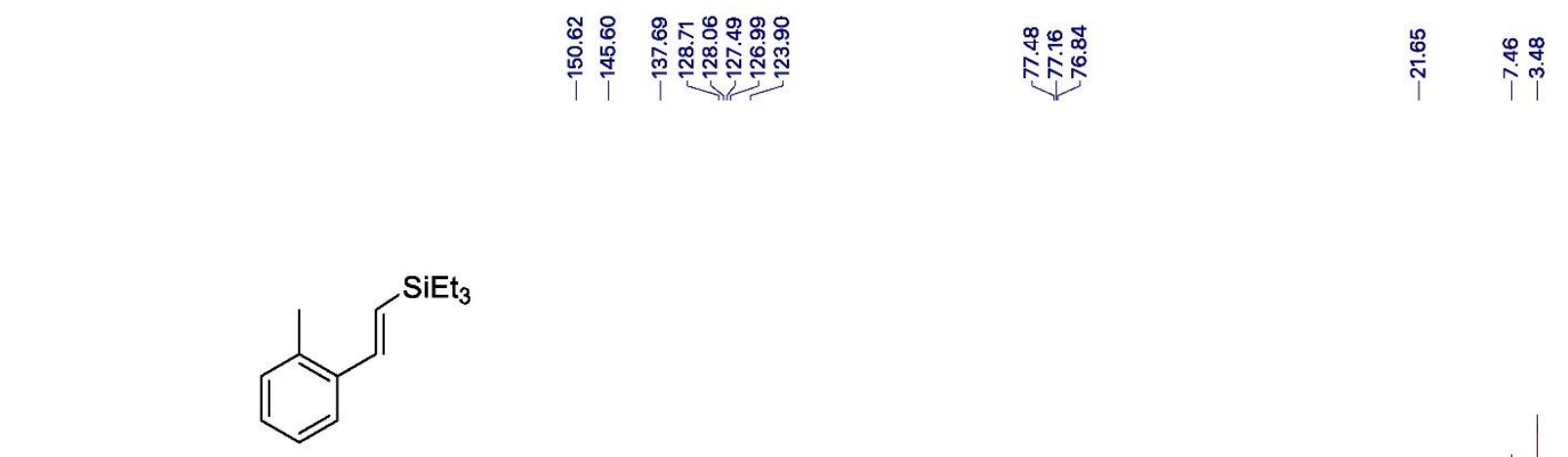

${ }^{13} \mathrm{C} \mathrm{NMR}\left(\mathrm{CDCl}_{3}, 100 \mathrm{MHz}, 298 \mathrm{~K}\right)$

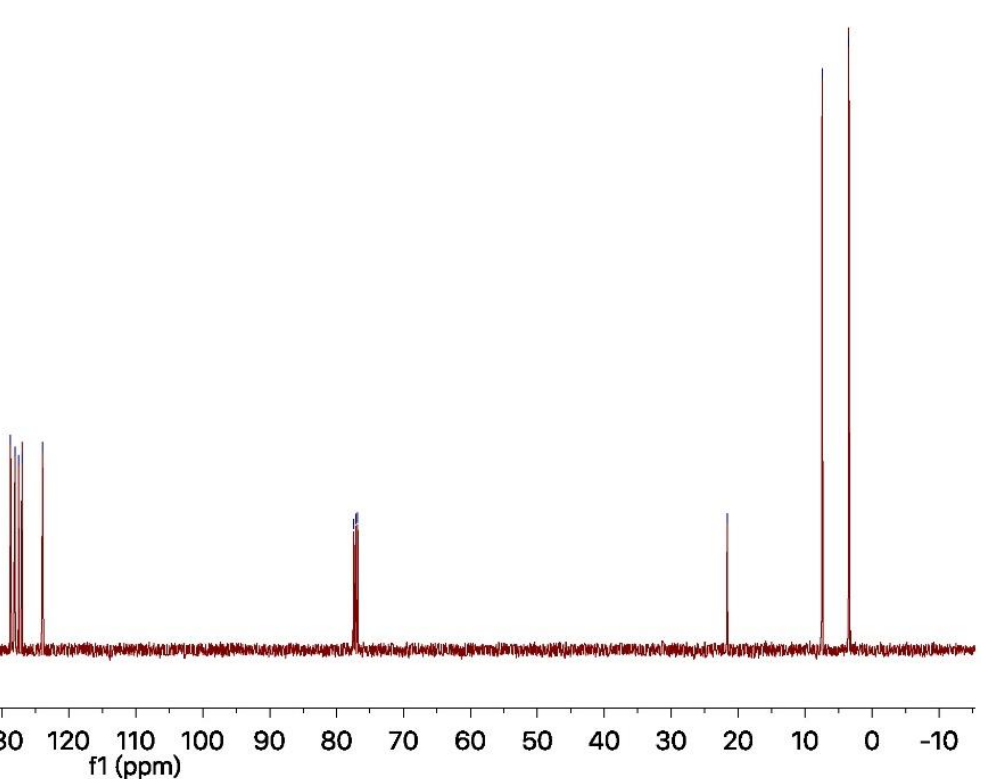

Figure S102. ${ }^{13} \mathrm{C}$ NMR spectrum of $\mathbf{P} 38$. 


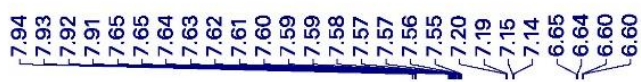

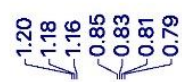
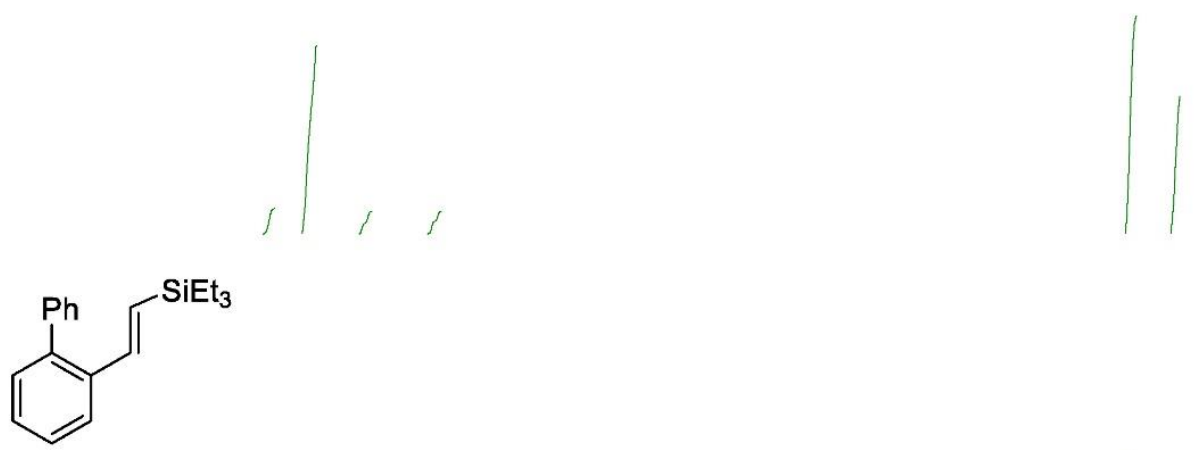

${ }^{1} \mathrm{H}$ NMR $\left(\mathrm{CDCl}_{3}, 400 \mathrm{MHz}, 296 \mathrm{~K}\right)$

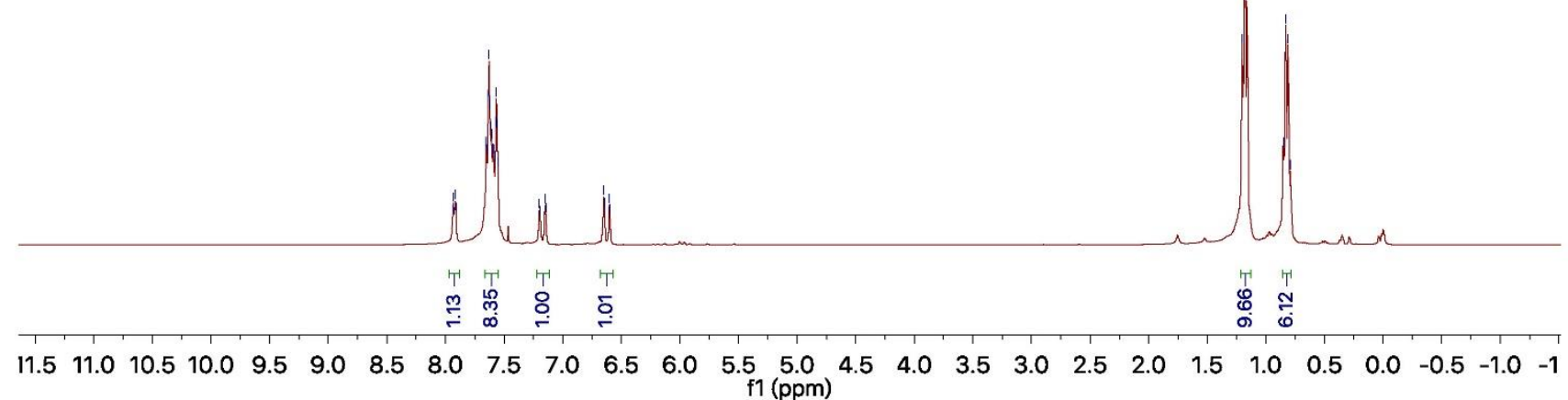

Figure S103. ${ }^{1} \mathrm{H}$ NMR spectrum of P39. 


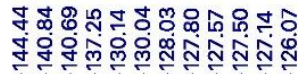

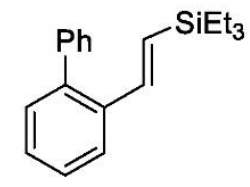

${ }^{13} \mathrm{C} \mathrm{NMR}\left(\mathrm{CDCl}_{3}, 100 \mathrm{MHz}, 296 \mathrm{~K}\right)$

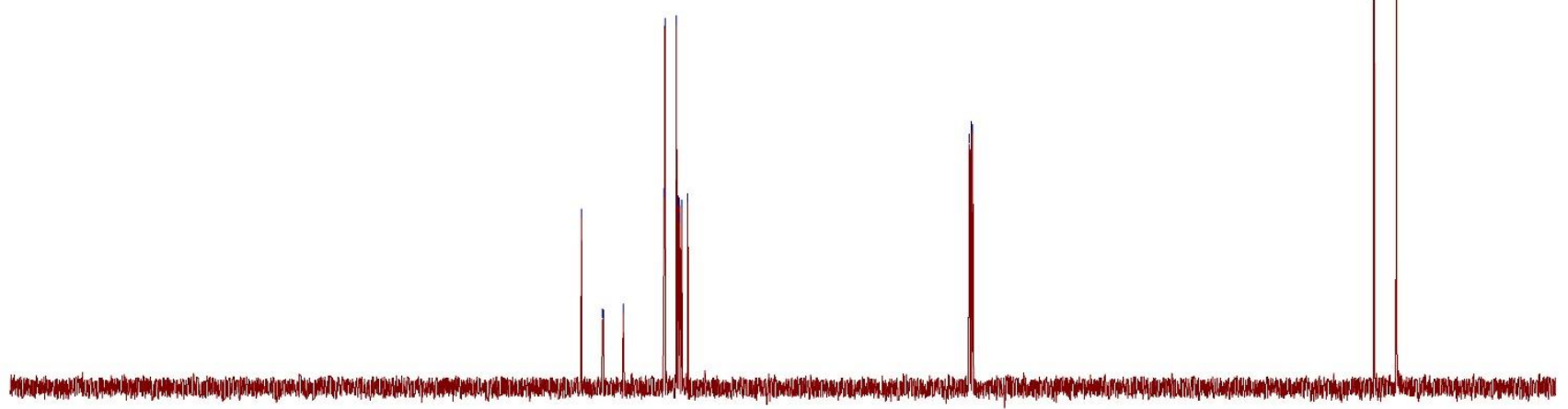

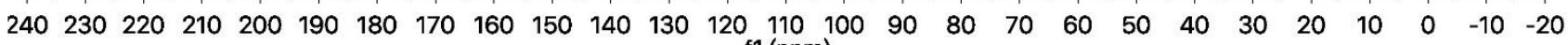
f1 (ppm)

Figure S104. ${ }^{13} \mathrm{C}$ NMR spectrum of P39. 


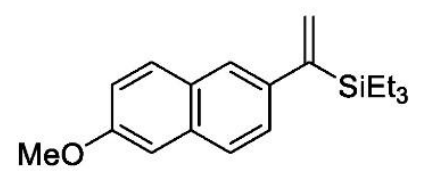

${ }^{1} \mathrm{H}$ NMR $\left(\mathrm{CDCl}_{3}, 400 \mathrm{MHz}, 298 \mathrm{~K}\right)$

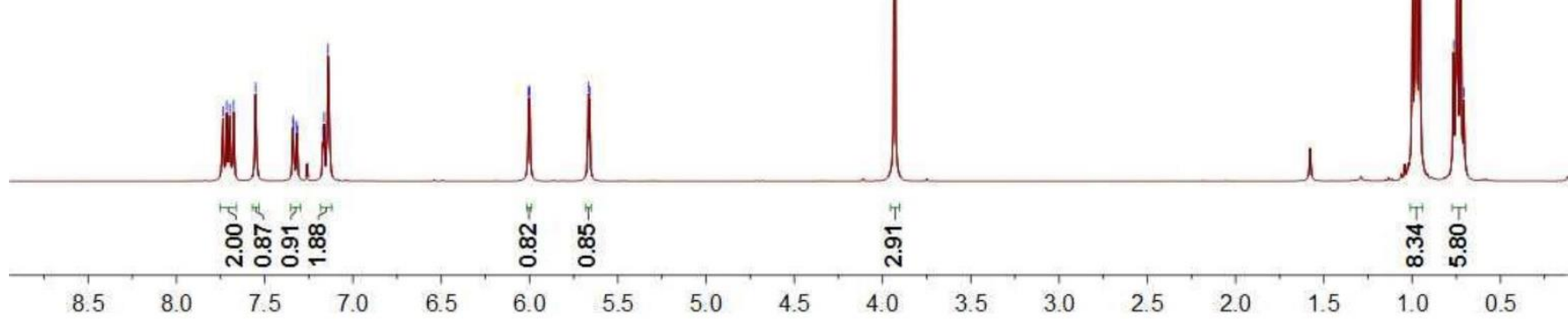

Figure S105. ${ }^{1} \mathrm{H}$ NMR spectrum of $\mathbf{P 4 0}$. 


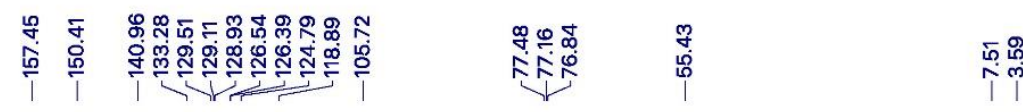

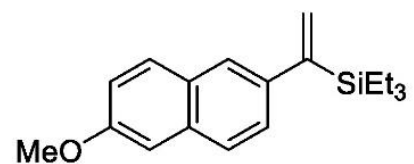

${ }^{13} \mathrm{C}$ NMR $\left(\mathrm{CDCl}_{3}, 100 \mathrm{MHz}, 298 \mathrm{~K}\right)$

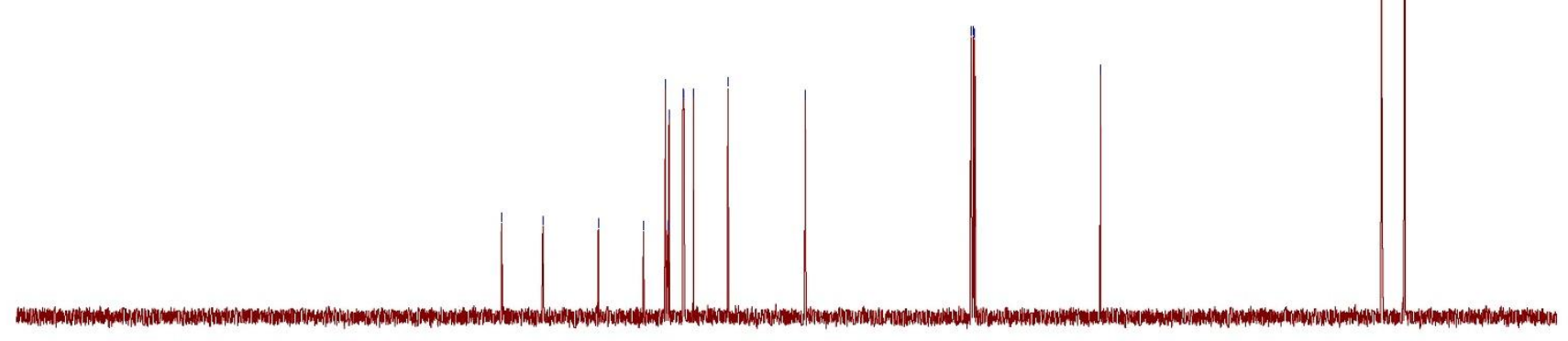

$\begin{array}{lllllllllllllllllllllllllll}10 & 230 & 220 & 210 & 200 & 190 & 180 & 170 & 160 & 150 & 140 & 130 & 120 & 110 & 100 & 90 & 80 & 70 & 60 & 50 & 40 & 30 & 20 & 10 & 0 & -10 & -2 \mathrm{C}\end{array}$

Figure S106. ${ }^{13} \mathrm{C}$ NMR spectrum of $\mathbf{P 4 0}$. 


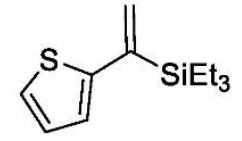

${ }^{1} \mathrm{H} \mathrm{NMR}\left(\mathrm{CDCl}_{3}, 400 \mathrm{MHz}, 298 \mathrm{~K}\right)$

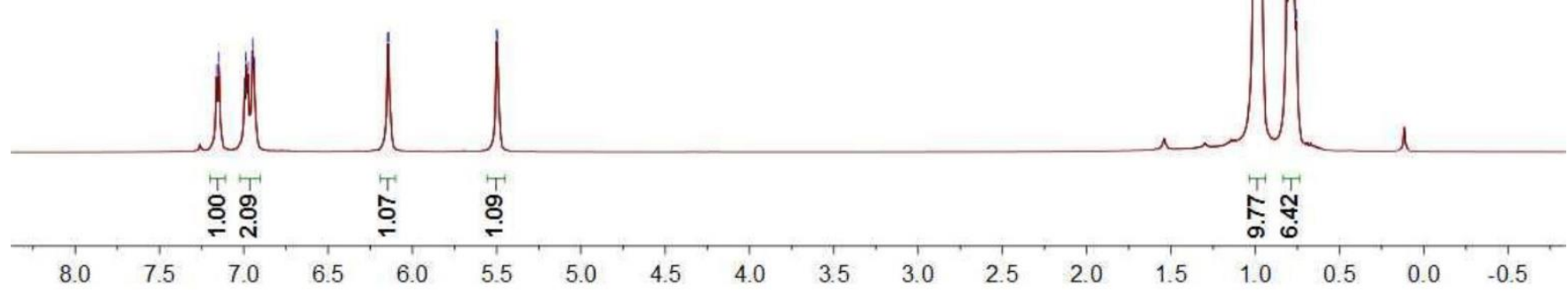

Figure S107. ${ }^{1} \mathrm{H}$ NMR spectrum of $\mathbf{P 4 1}$. 


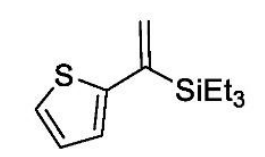

${ }^{13} \mathrm{C} \mathrm{NMR}\left(\mathrm{CDCl}_{3}, 100 \mathrm{MHz}, 298 \mathrm{~K}\right)$

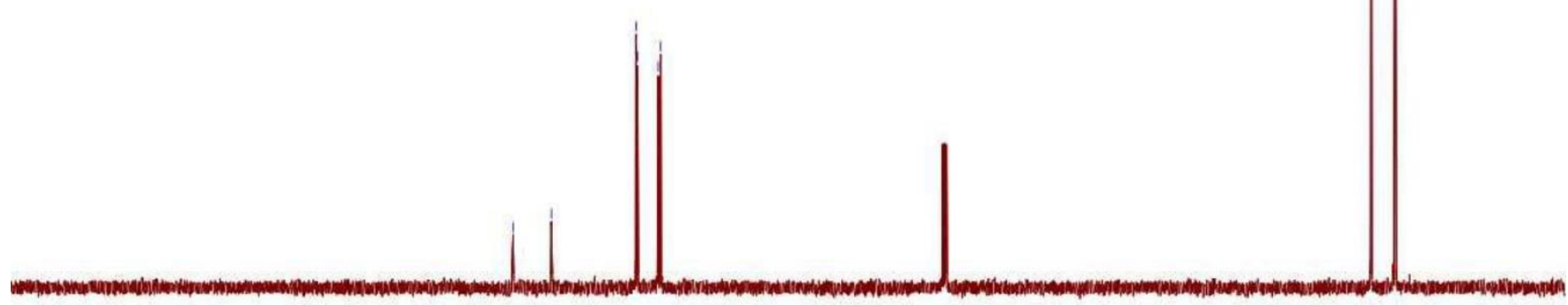

Figure S108. ${ }^{13} \mathrm{C}$ NMR spectrum of $\mathbf{P 4 1}$. 


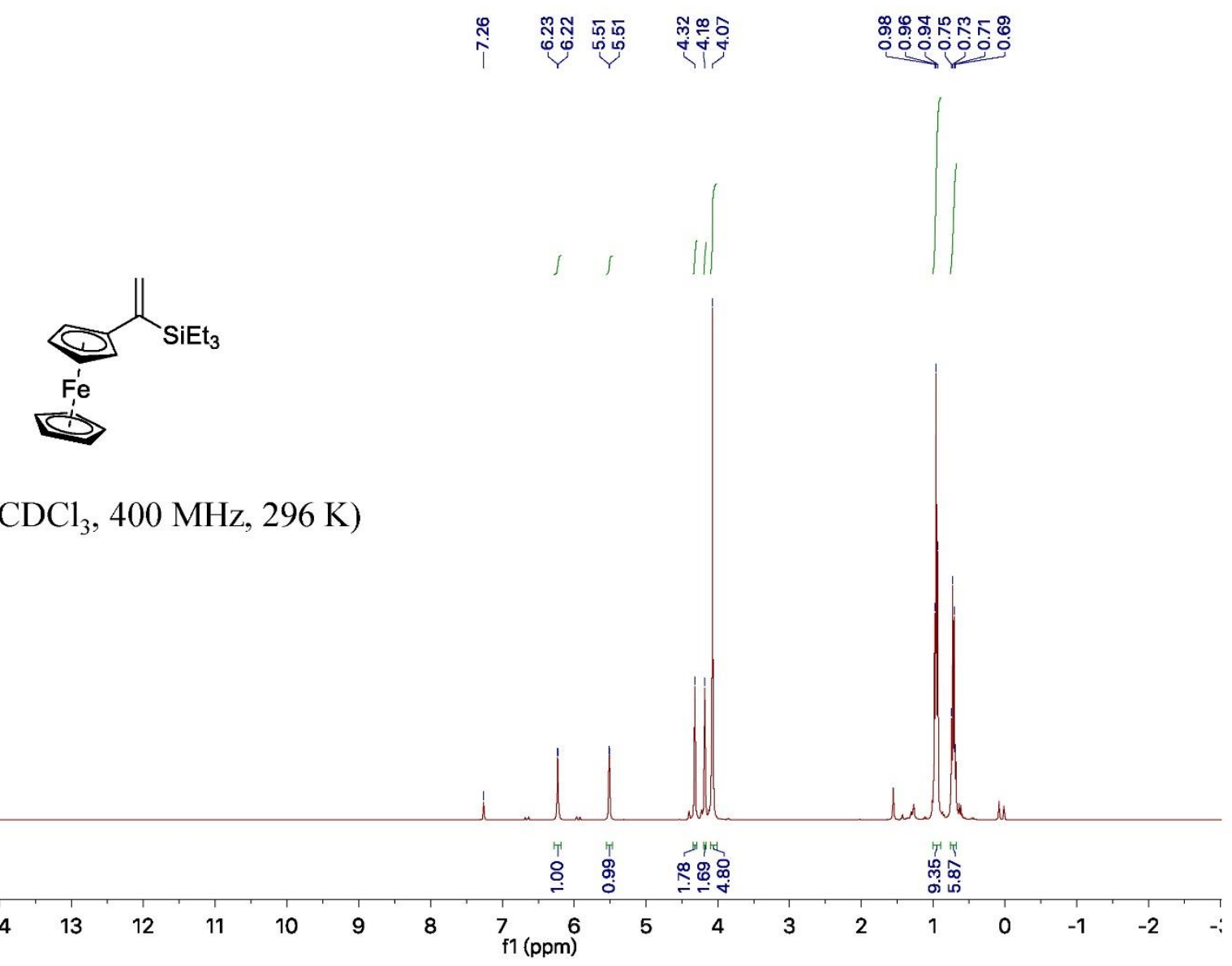

Figure S109. ${ }^{1} \mathrm{H}$ NMR spectrum of $\mathbf{P 4 2}$. 


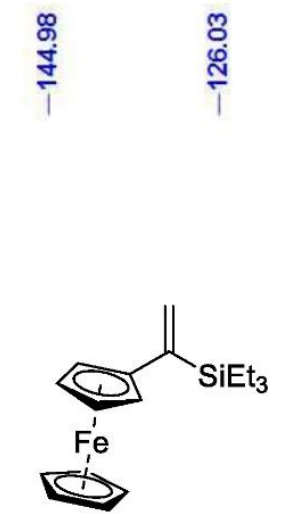

${ }^{13} \mathrm{C}$ NMR $\left(\mathrm{CDCl}_{3}, 100 \mathrm{MHz}, 296 \mathrm{~K}\right)$
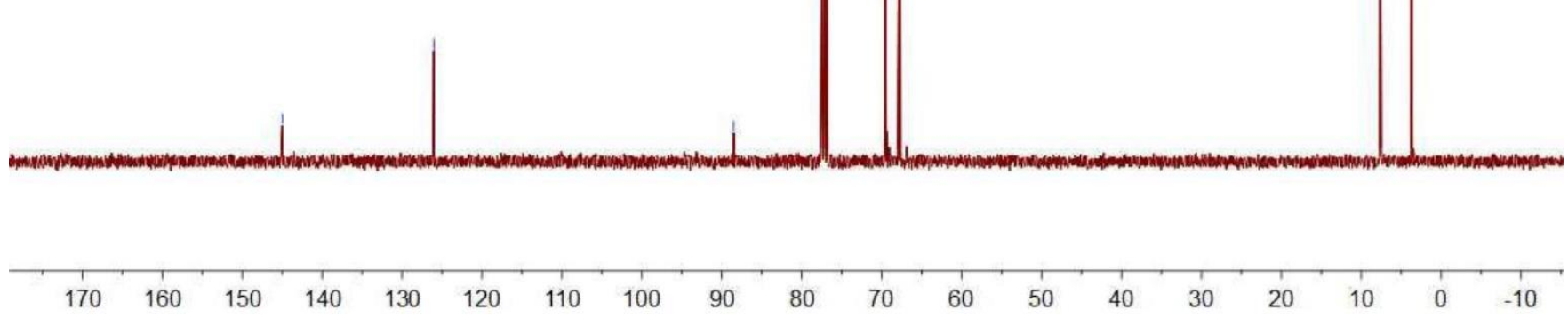

Figure S110. ${ }^{13} \mathrm{C}$ NMR spectrum of $\mathbf{P 4 2}$. 


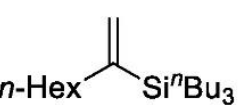

${ }^{1} \mathrm{H} \mathrm{NMR}\left(\mathrm{CDCl}_{3}, 400 \mathrm{MHz}, 294 \mathrm{~K}\right)$

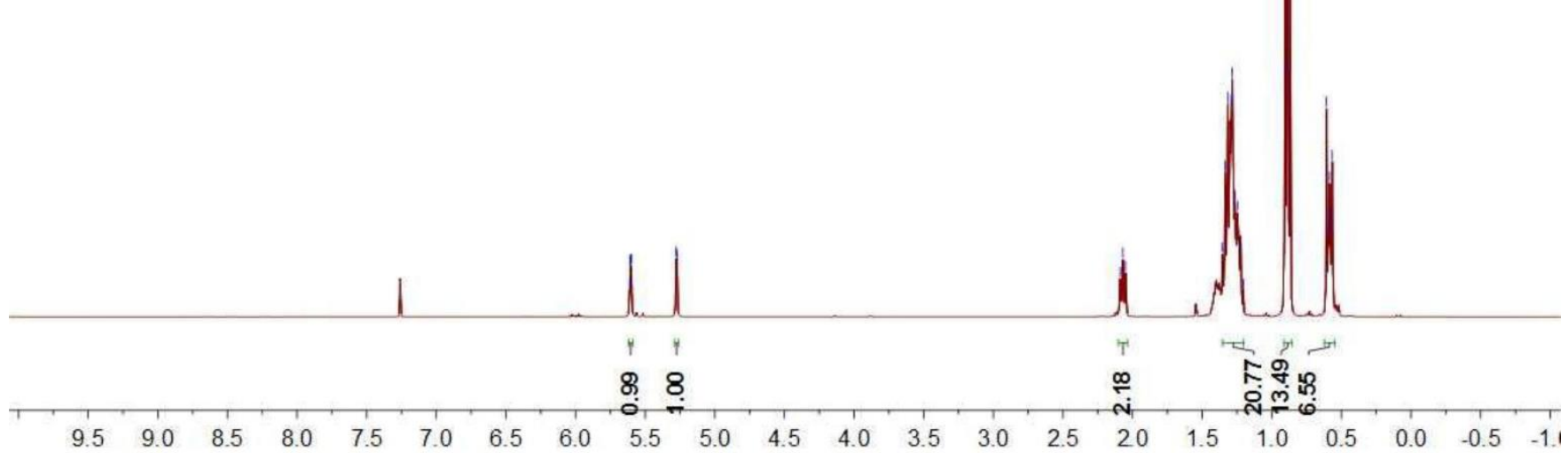

Figure S111. ${ }^{1} \mathrm{H}$ NMR spectrum of $\mathbf{P 4 3}$. 

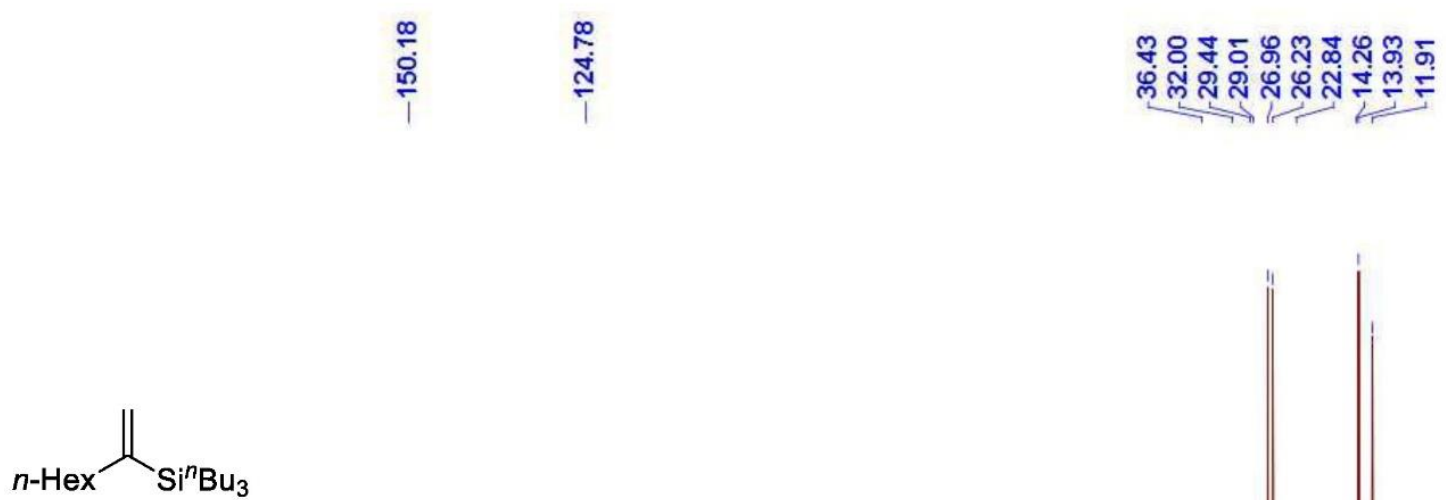

${ }^{13} \mathrm{C} \mathrm{NMR}\left(\mathrm{CDCl}_{3}, 100 \mathrm{MHz}, 298 \mathrm{~K}\right)$
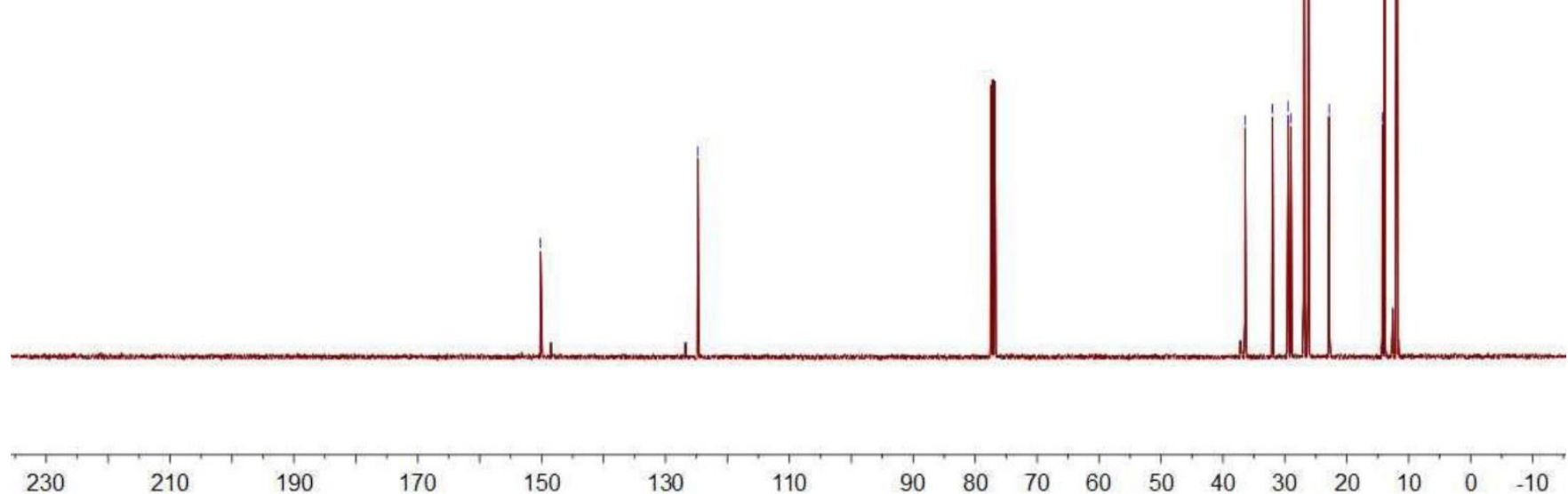

110
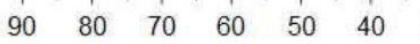

Figure S112. ${ }^{13} \mathrm{C}$ NMR spectrum of $\mathbf{P 4 3}$. 


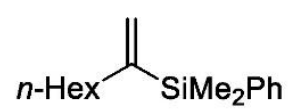

'H NMR (CDCl $3,400 \mathrm{MHz}, 298 \mathrm{~K})$

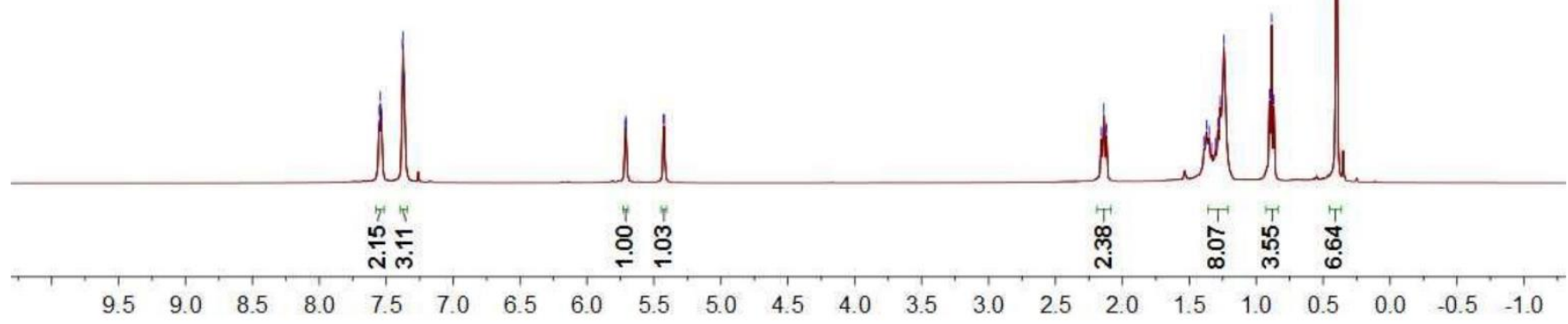

Figure S113. ${ }^{1} \mathrm{H}$ NMR spectrum of $\mathbf{P 4 4}$. 


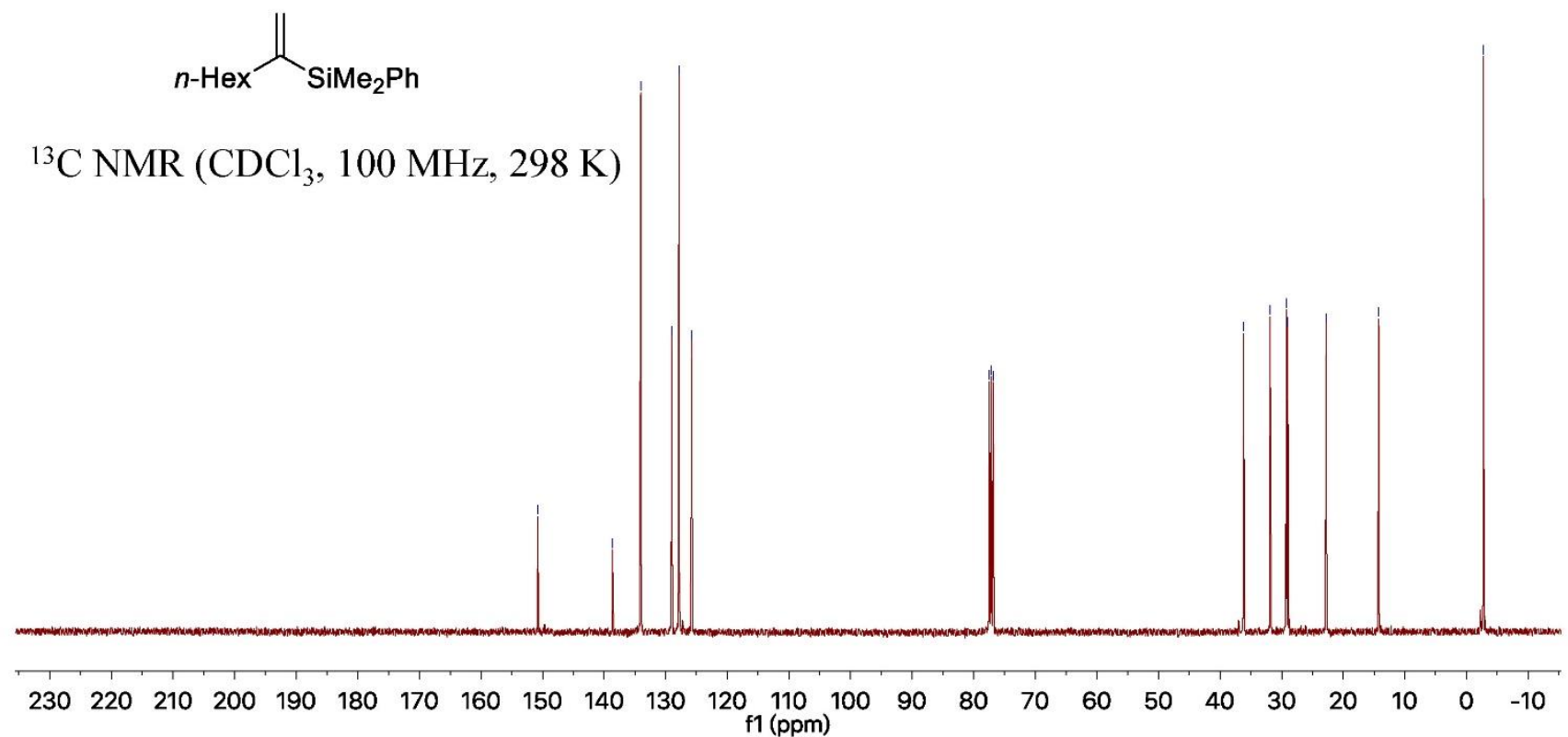

Figure S114. ${ }^{13} \mathrm{C}$ NMR spectrum of $\mathbf{P 4 4}$. 


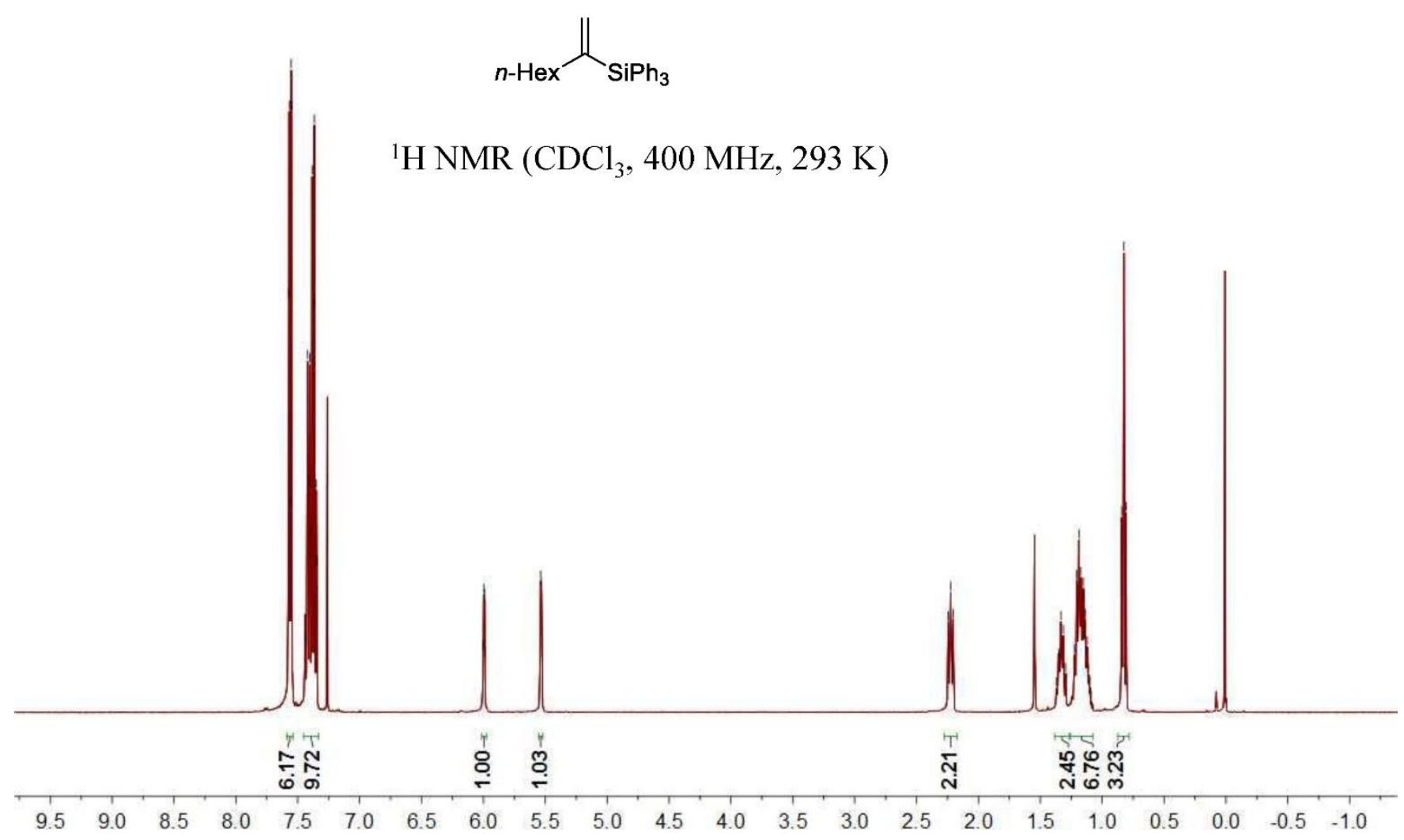

Figure S115. ${ }^{1} \mathrm{H}$ NMR spectrum of $\mathbf{P 4 5}$. 


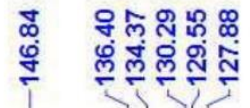

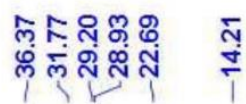

$\underset{n-\mathrm{Hex}}{\mathrm{SiPh}_{3}}$

${ }^{13} \mathrm{C}$ NMR $\left(\mathrm{CDCl}_{3}, 100 \mathrm{MHz}, 294 \mathrm{~K}\right)$
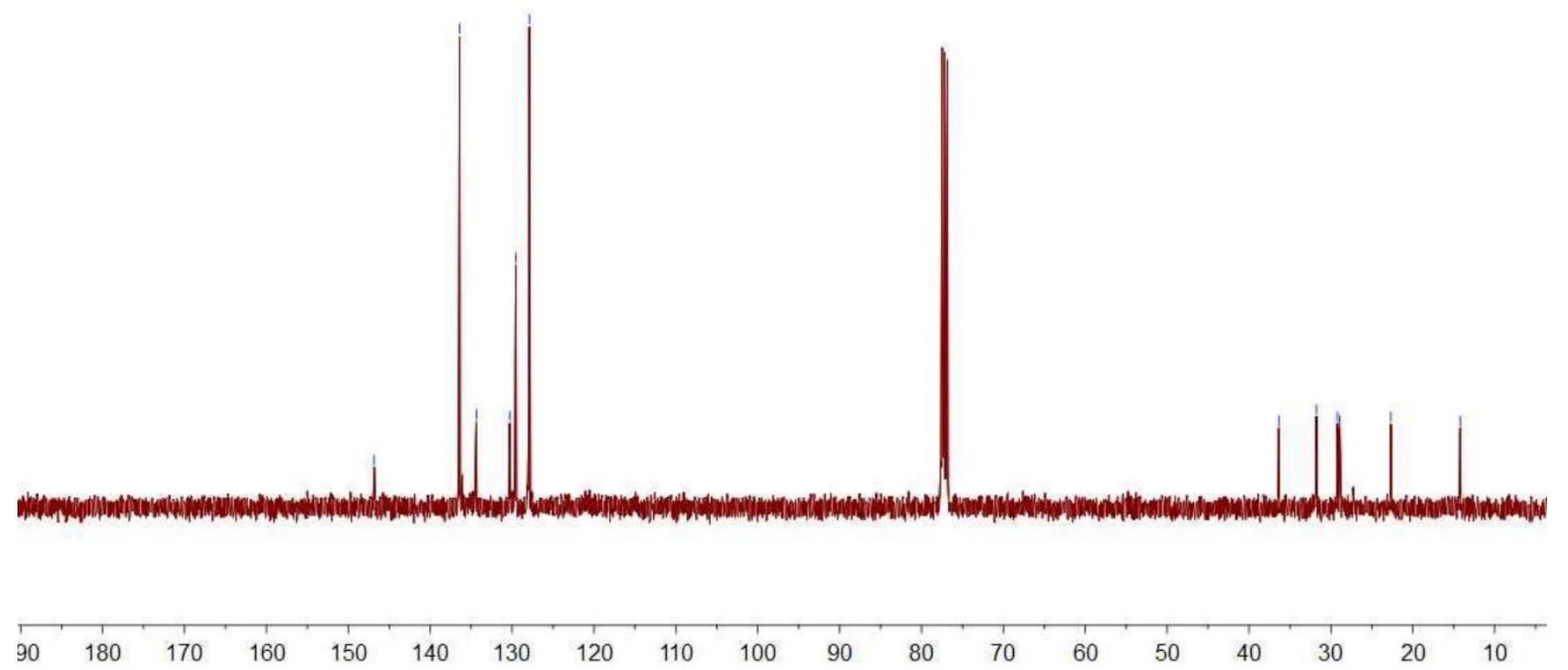

Figure S116. ${ }^{13} \mathrm{C}$ NMR spectrum of $\mathbf{P 4 5}$. 


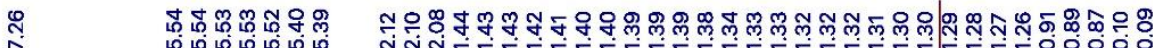

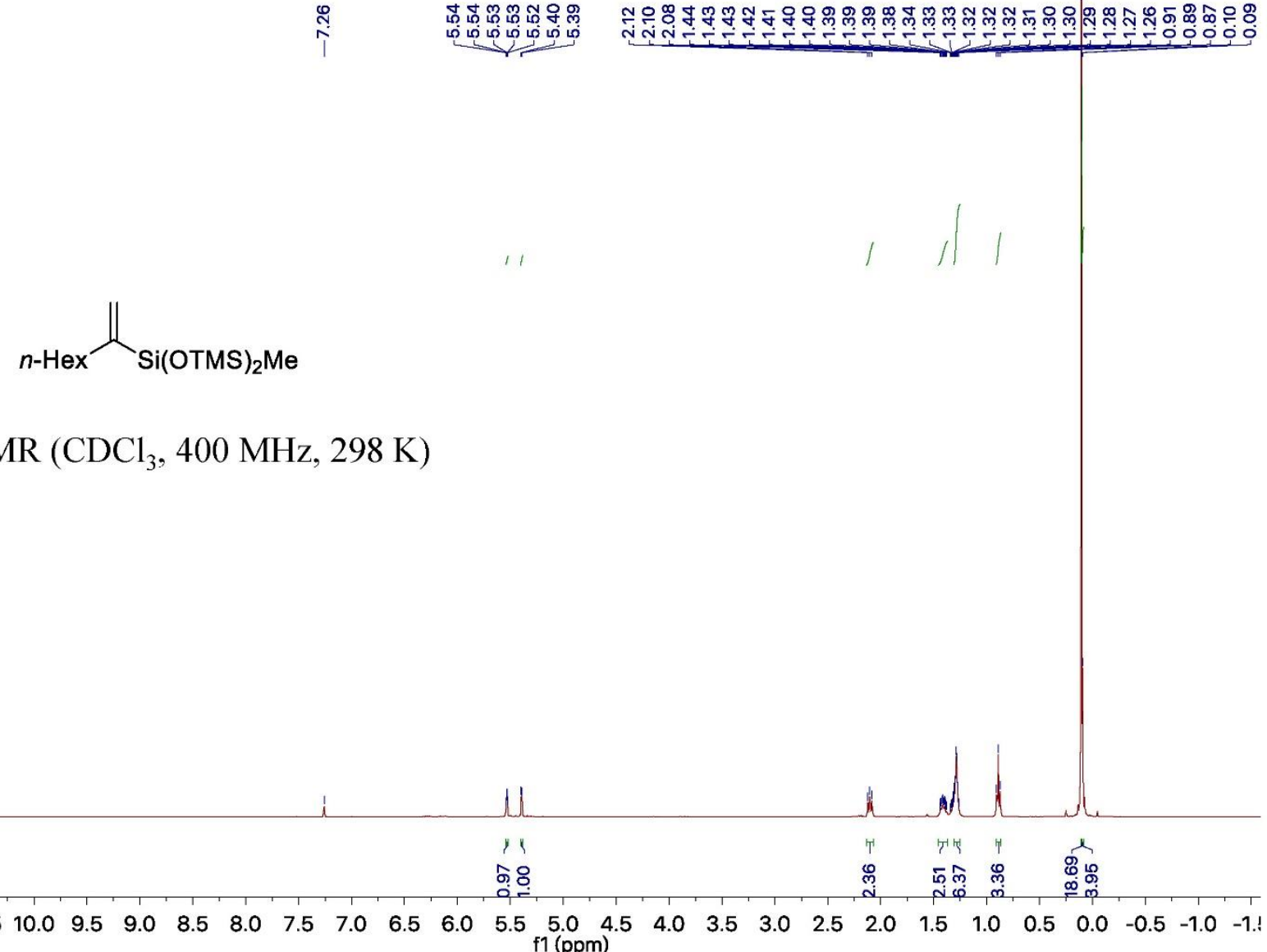

Figure S117. ${ }^{1} \mathrm{H}$ NMR spectrum of $\mathbf{P 4 6}$. 

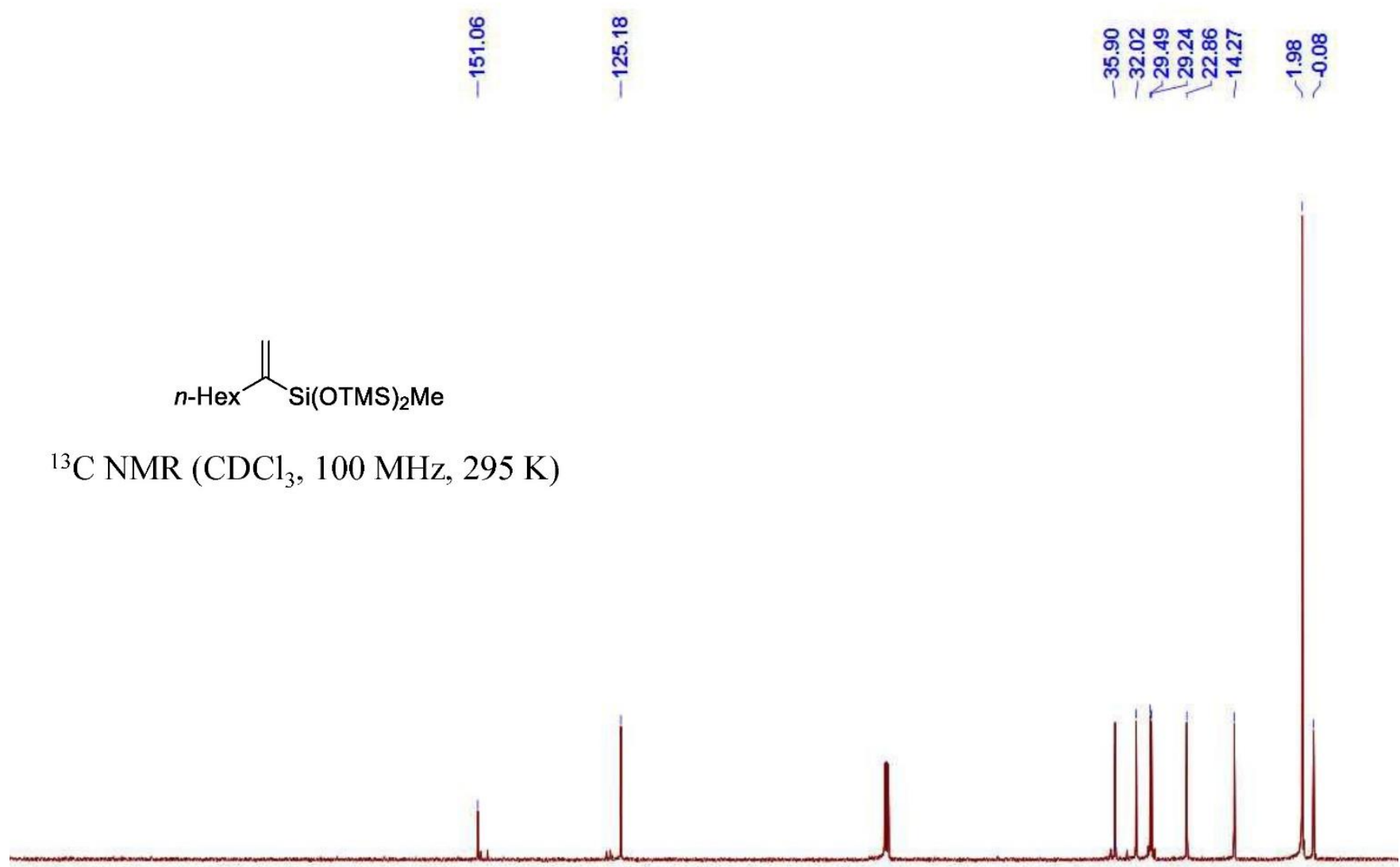

${ }^{13} \mathrm{C} \mathrm{NMR}\left(\mathrm{CDCl}_{3}, 100 \mathrm{MHz}, 295 \mathrm{~K}\right)$

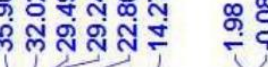

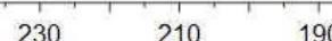

150

Figure S118. ${ }^{13} \mathrm{C}$ NMR spectrum of $\mathbf{P 4 6}$. 


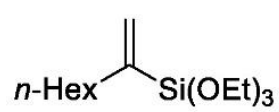

${ }^{1} \mathrm{H} \mathrm{NMR}\left(\mathrm{CDCl}_{3}, 400 \mathrm{MHz}, 293 \mathrm{~K}\right)$

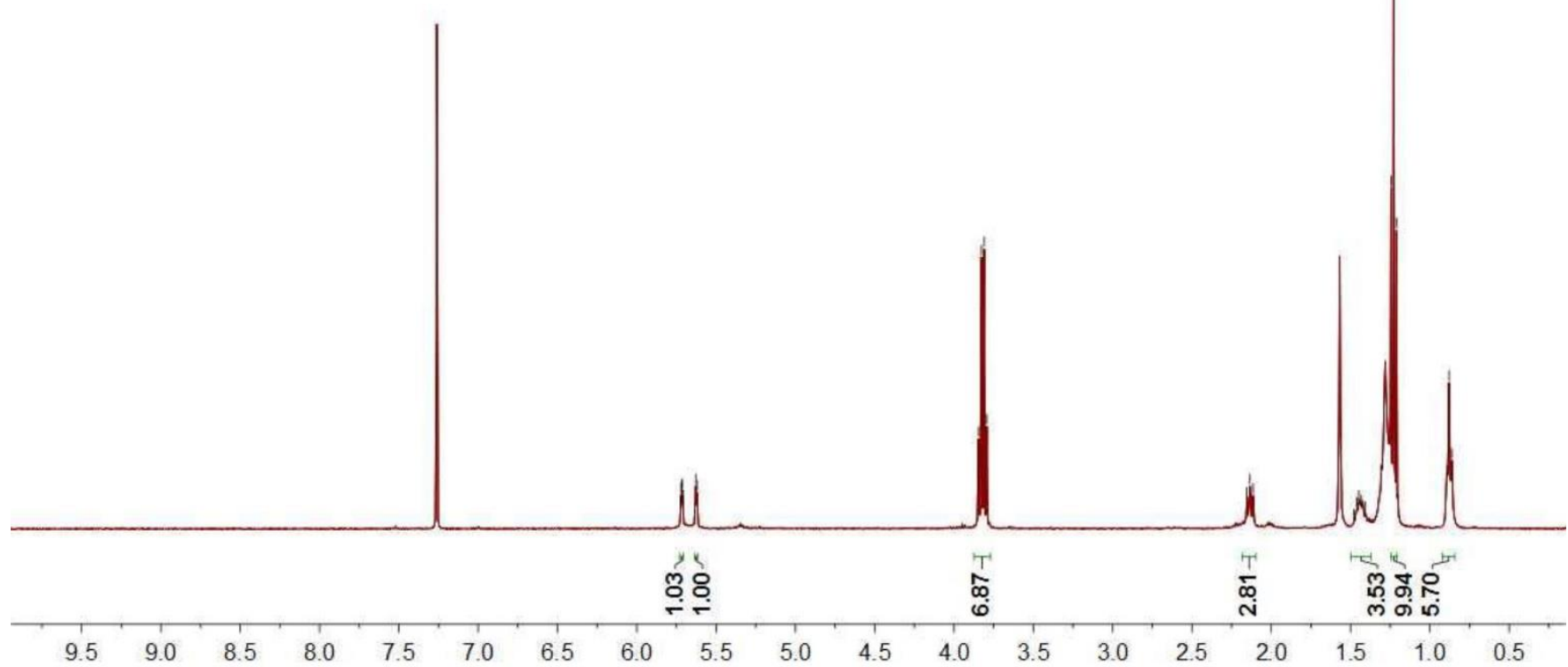

Figure S119. ${ }^{1} \mathrm{H}$ NMR spectrum of $\mathbf{P 4 7}$. 


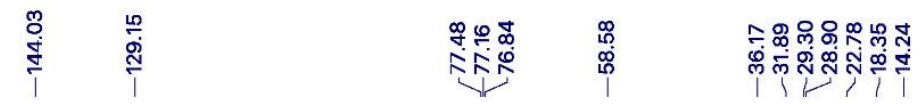

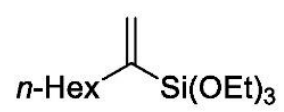

${ }^{13} \mathrm{C} \mathrm{NMR}\left(\mathrm{CDCl}_{3}, 100 \mathrm{MHz}, 298 \mathrm{~K}\right)$

$\begin{array}{lllllllllllllllllllllllll}230 & 220 & 210 & 200 & 190 & 180 & 170 & 160 & 150 & 140 & 130 & 120 & 110 & 100 & 90 & 80 & 70 & 60 & 50 & 40 & 30 & 20 & 10 & 0 & -10\end{array}$

Figure S120. ${ }^{13} \mathrm{C}$ NMR spectrum of $\mathbf{P 4 7}$. 


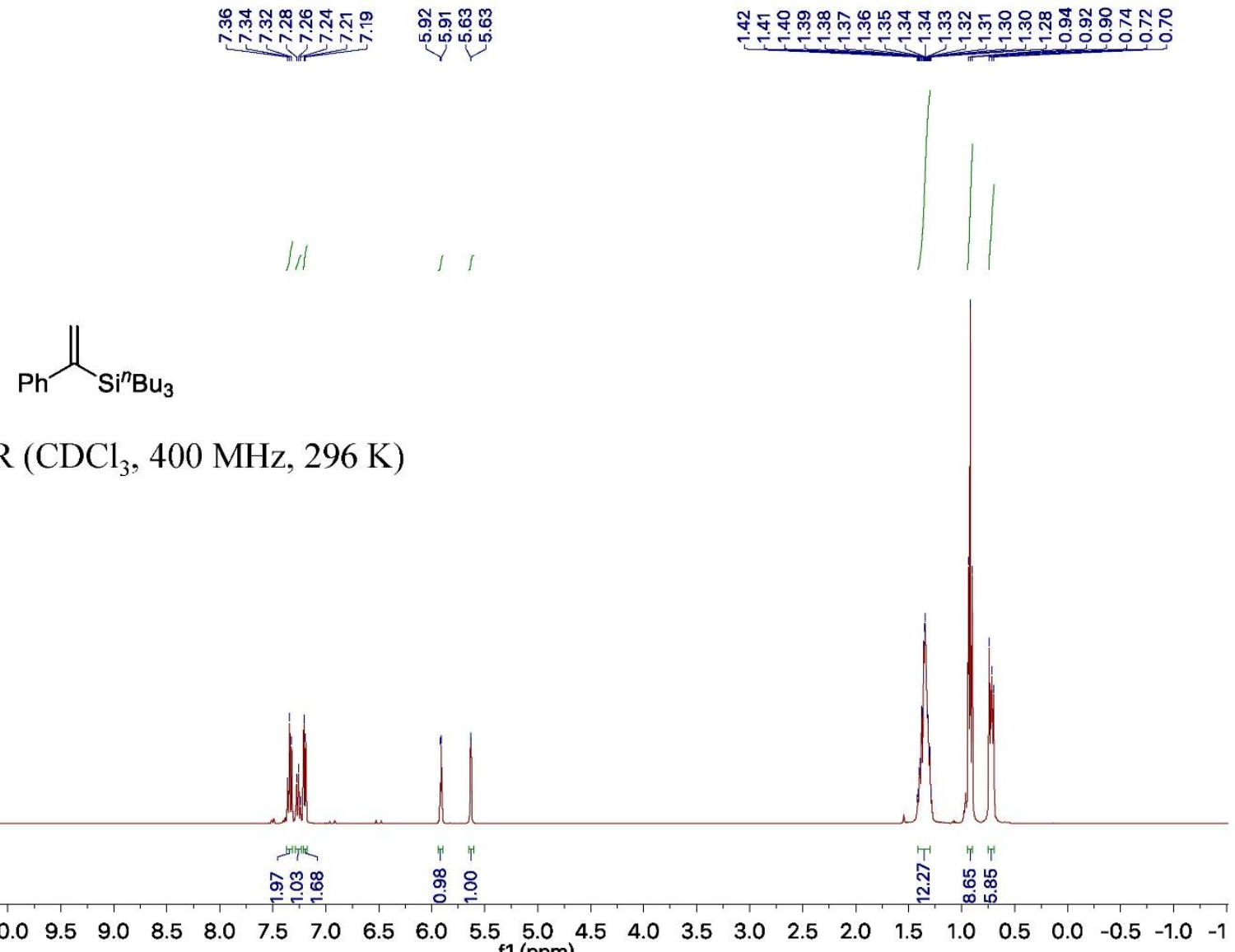

Figure S121. ${ }^{1} \mathrm{H}$ NMR spectrum of $\mathbf{P 4 8}$. 


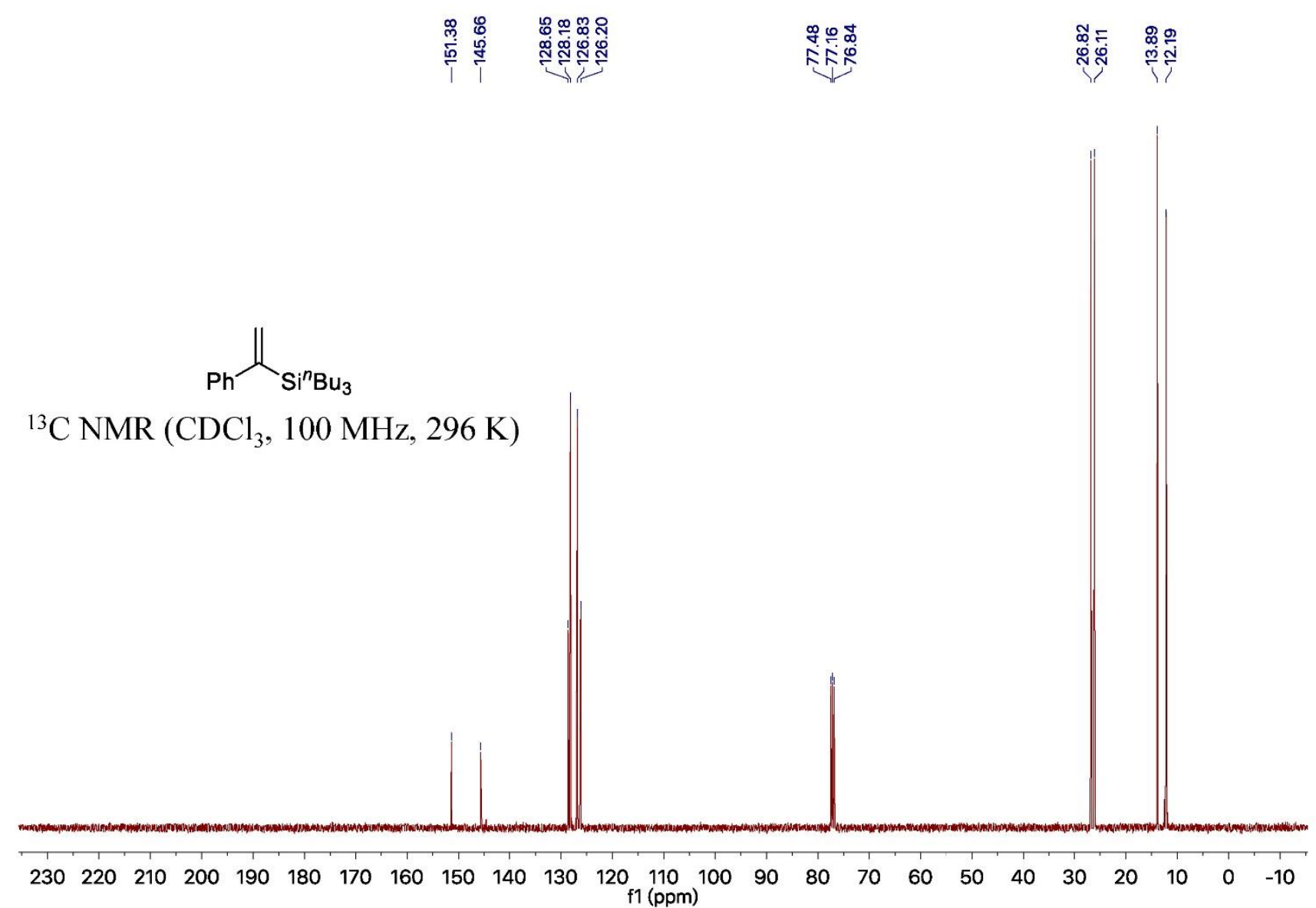

Figure S122. ${ }^{13} \mathrm{C}$ NMR spectrum of $\mathbf{P} 48$. 


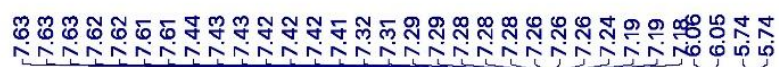

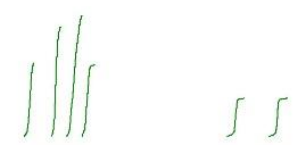

\author{
$\mathrm{Ph}_{\mathrm{SiMe}_{2} \mathrm{Ph}}$
}

${ }^{1} \mathrm{H}$ NMR $\left(\mathrm{CDCl}_{3}, 400 \mathrm{MHz}, 296 \mathrm{~K}\right)$

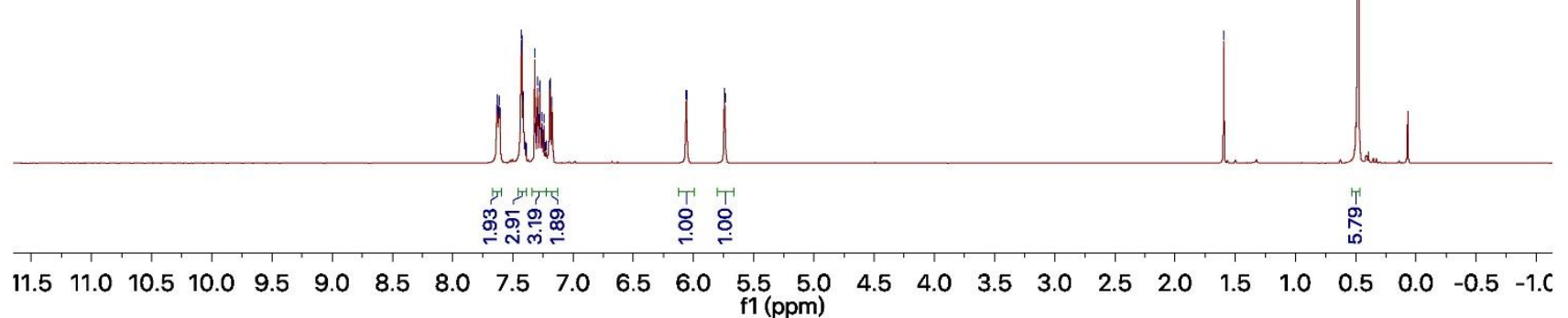

Figure S123. ${ }^{1} \mathrm{H}$ NMR spectrum of $\mathbf{P 4 9}$. 


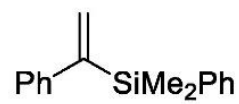

${ }^{13} \mathrm{C} \mathrm{NMR}\left(\mathrm{CDCl}_{3}, 100 \mathrm{MHz}, 296 \mathrm{~K}\right)$

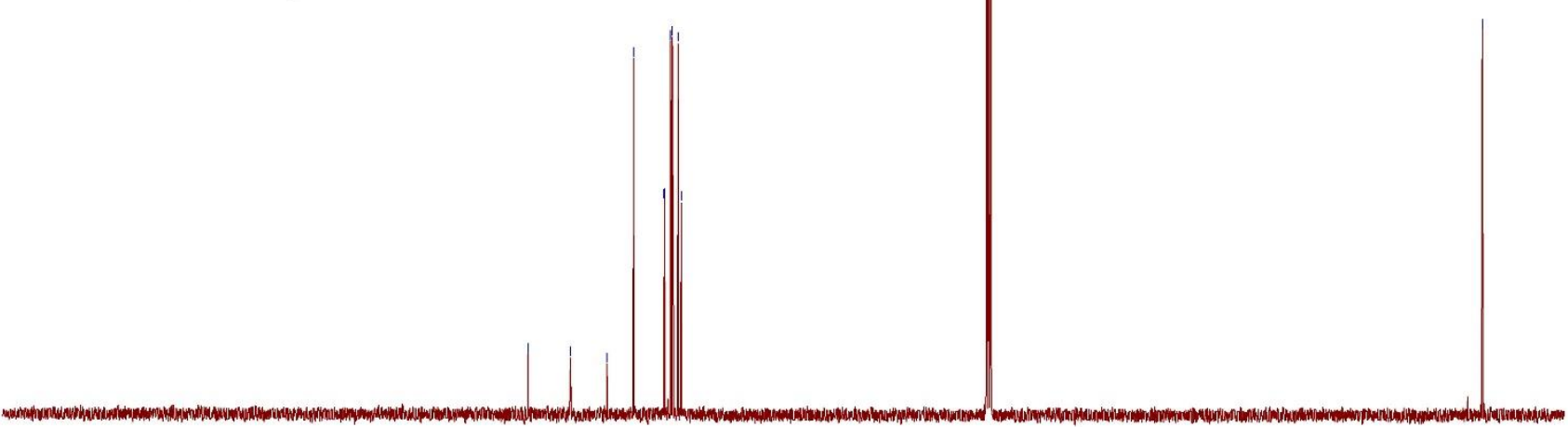

$\begin{array}{lllllllllllllllllllllllll}230 & 220 & 210 & 200 & 190 & 180 & 170 & 160 & 150 & 140 & 130 & 120 & 110 & 100 & 90 & 80 & 70 & 60 & 50 & 40 & 30 & 20 & 10 & 0 & -10\end{array}$

Figure S124. ${ }^{13} \mathrm{C}$ NMR spectrum of $\mathbf{P 4 9}$. 


$$
\mathrm{Ph}_{\mathrm{SiMe}_{2} \mathrm{Ph}}
$$

${ }^{29} \mathrm{Si} \mathrm{NMR}\left(79 \mathrm{MHz}, \mathrm{CDCl}_{3}, 297 \mathrm{~K}\right)$

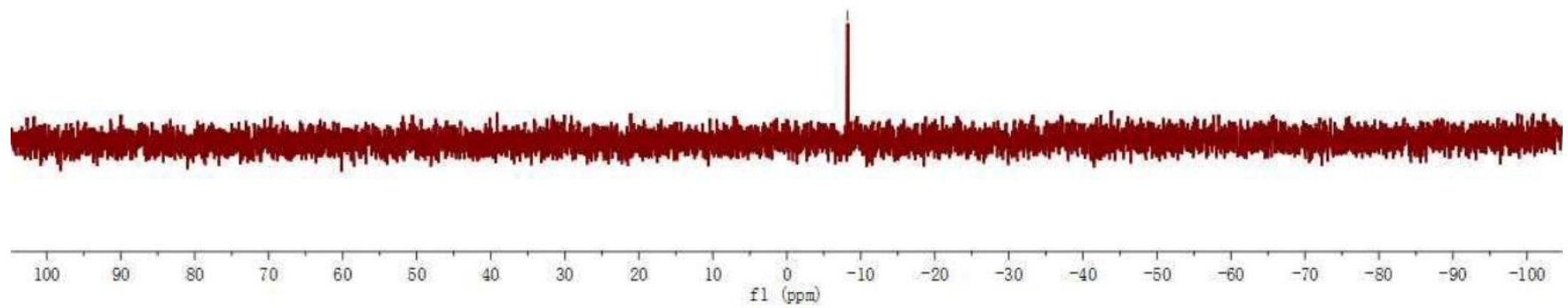

Figure S125. ${ }^{29} \mathrm{Si}$ NMR spectrum of $\mathbf{P 4 9}$. 


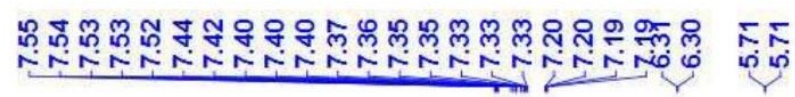

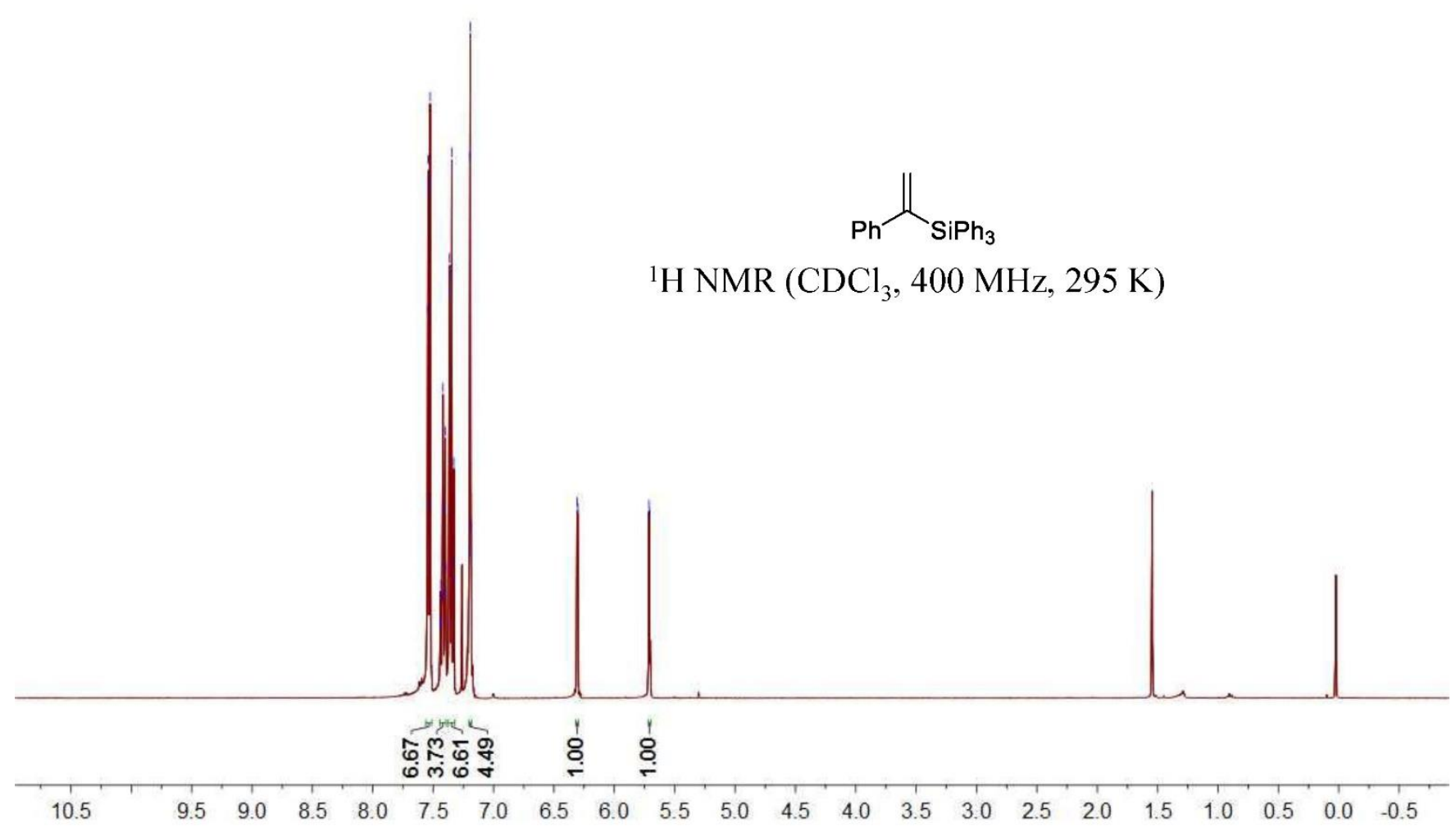

Figure S126. ${ }^{1} \mathrm{H}$ NMR spectrum of P50. 


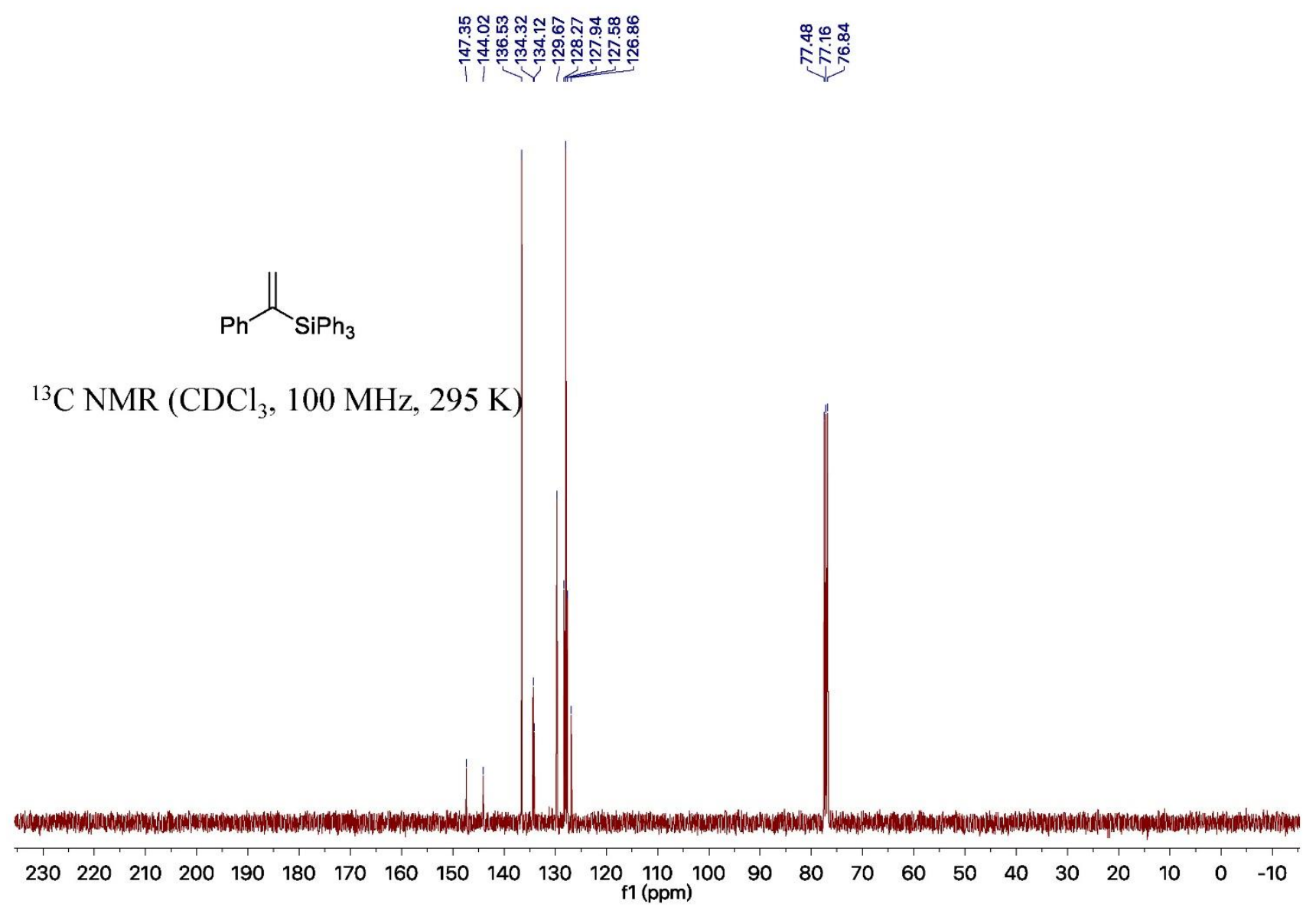

Figure S127. ${ }^{13} \mathrm{C}$ NMR spectrum of $\mathbf{P 5 0}$. 


$$
{ }_{\mathrm{Ph}} \mathrm{L}_{\mathrm{SiPh}_{3}}
$$

${ }^{29} \mathrm{Si} \mathrm{NMR}\left(79 \mathrm{MHz}, \mathrm{CDCl}_{3}, 297 \mathrm{~K}\right)$

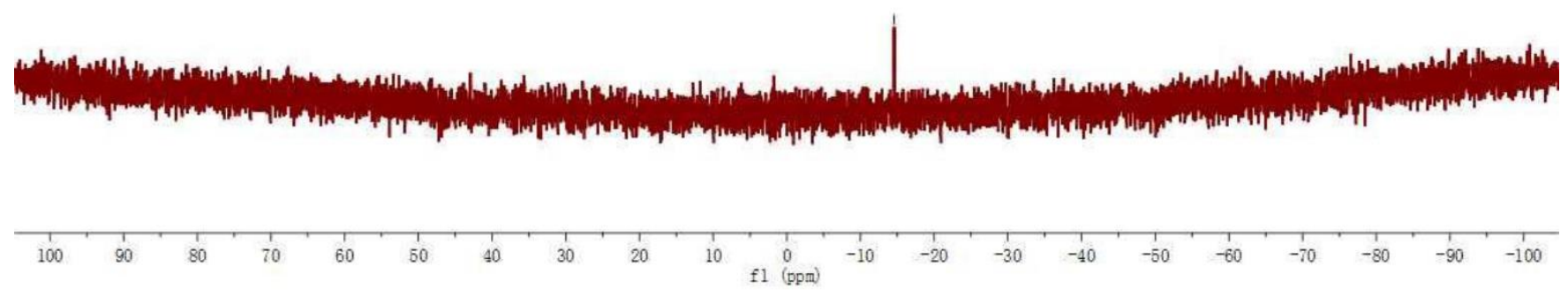

Figure S128. ${ }^{29}$ Si NMR spectrum of P50. 


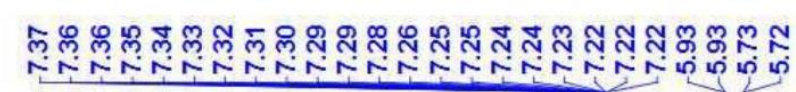

$\mathrm{Ph}_{\mathrm{Si}(\mathrm{OTMS})_{2} \mathrm{Me}}$

${ }^{1} \mathrm{H} \mathrm{NMR}\left(\mathrm{CDCl}_{3}, 400 \mathrm{MHz}, 298 \mathrm{~K}\right)$

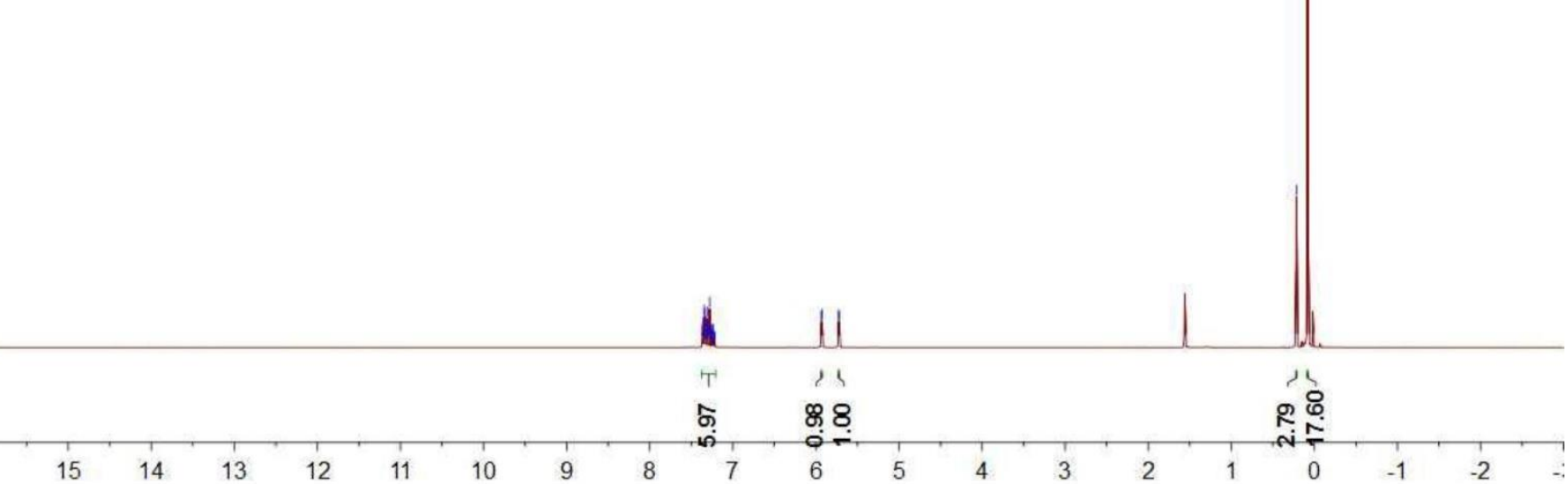

Figure S129. ${ }^{1} \mathrm{H}$ NMR spectrum of $\mathbf{P 5 1}$. 
${ }^{13} \mathrm{C} \mathrm{NMR}\left(\mathrm{CDCl}_{3}, 100 \mathrm{MHz}, 295 \mathrm{~K}\right)$

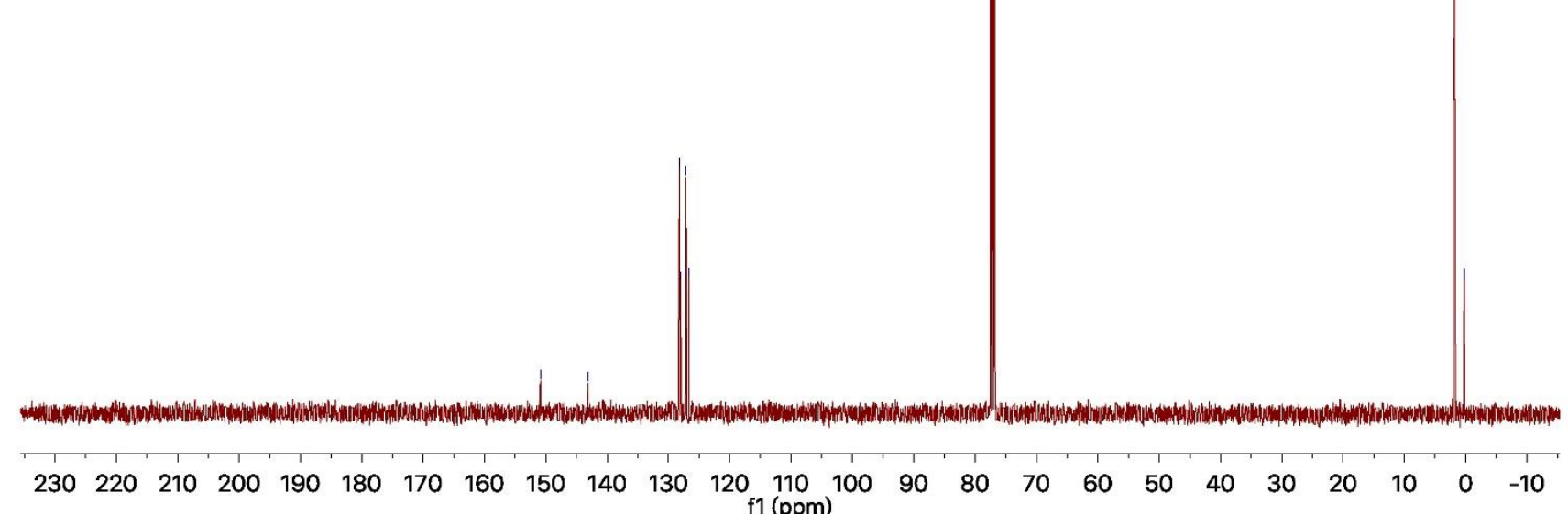

Figure S130. ${ }^{13} \mathrm{C}$ NMR spectrum of $\mathbf{P 5 1}$. 
$\mathrm{Ph}_{\mathrm{Si}(\mathrm{OEt})_{3}}$

'H NMR (CDCl 3 , 400 MHz, 298 K)

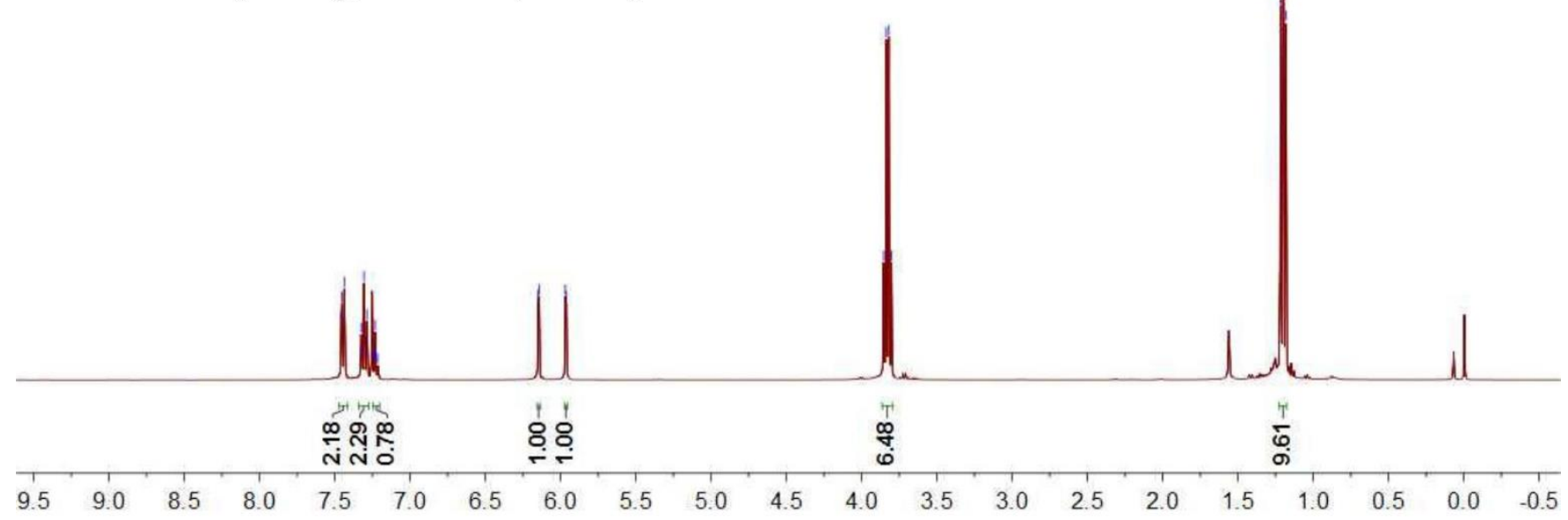

Figure S131. ${ }^{1} \mathrm{H}$ NMR spectrum of $\mathbf{P 5 2}$. 


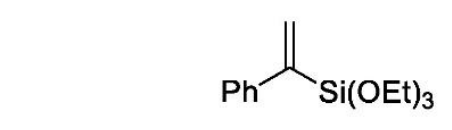

${ }^{13} \mathrm{C} \mathrm{NMR}\left(\mathrm{CDCl}_{3}, 100 \mathrm{MHz}, 298 \mathrm{~K}\right)$

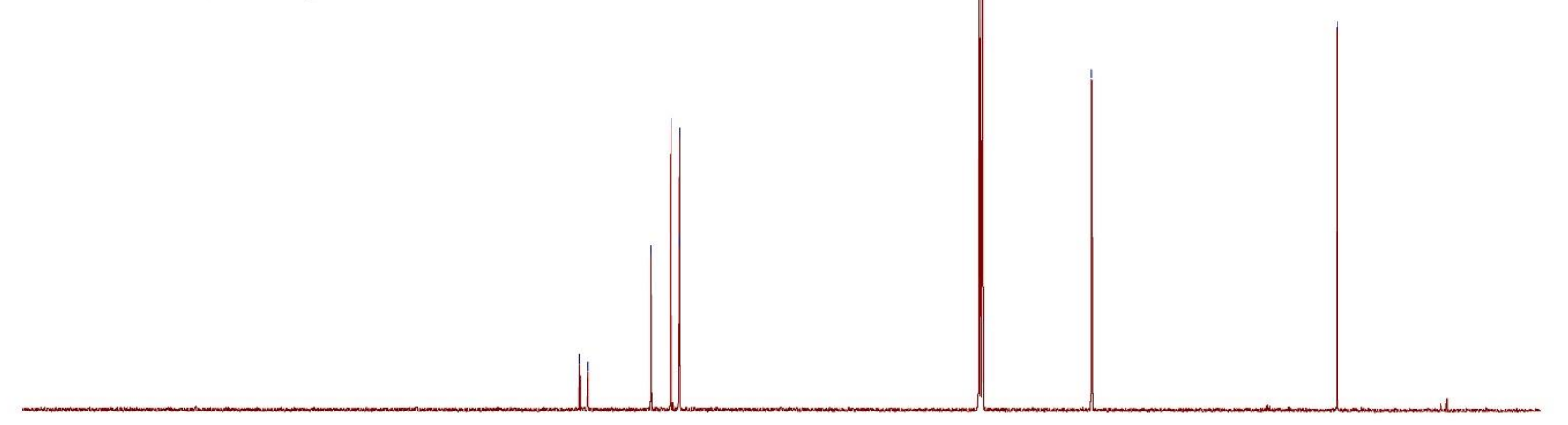

$\begin{array}{llllllllllllllllllllllllll}230 & 220 & 210 & 200 & 190 & 180 & 170 & 160 & 150 & 140 & 130 & 120 & 110 & 100 & 90 & 80 & 70 & 60 & 50 & 40 & 30 & 20 & 10 & 0 & -10\end{array}$

Figure S132. ${ }^{13} \mathrm{C}$ NMR spectrum of $\mathbf{P 5 2}$. 


$$
\overbrace{\mathrm{Me}_{2} \mathrm{Cl}}
$$

${ }^{1} \mathrm{H}$ NMR $\left(\mathrm{C}_{6} \mathrm{D}_{6}, 400 \mathrm{MHz}, 294 \mathrm{~K}\right)$

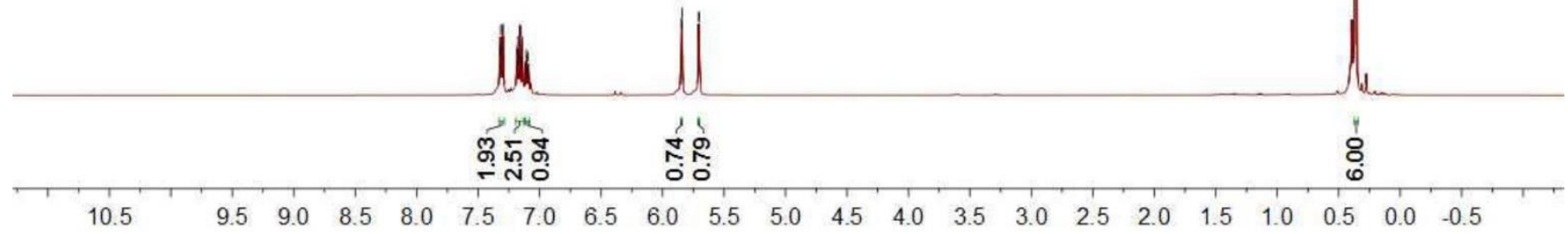

Figure S133. ${ }^{1} \mathrm{H}$ NMR spectrum of P53. 

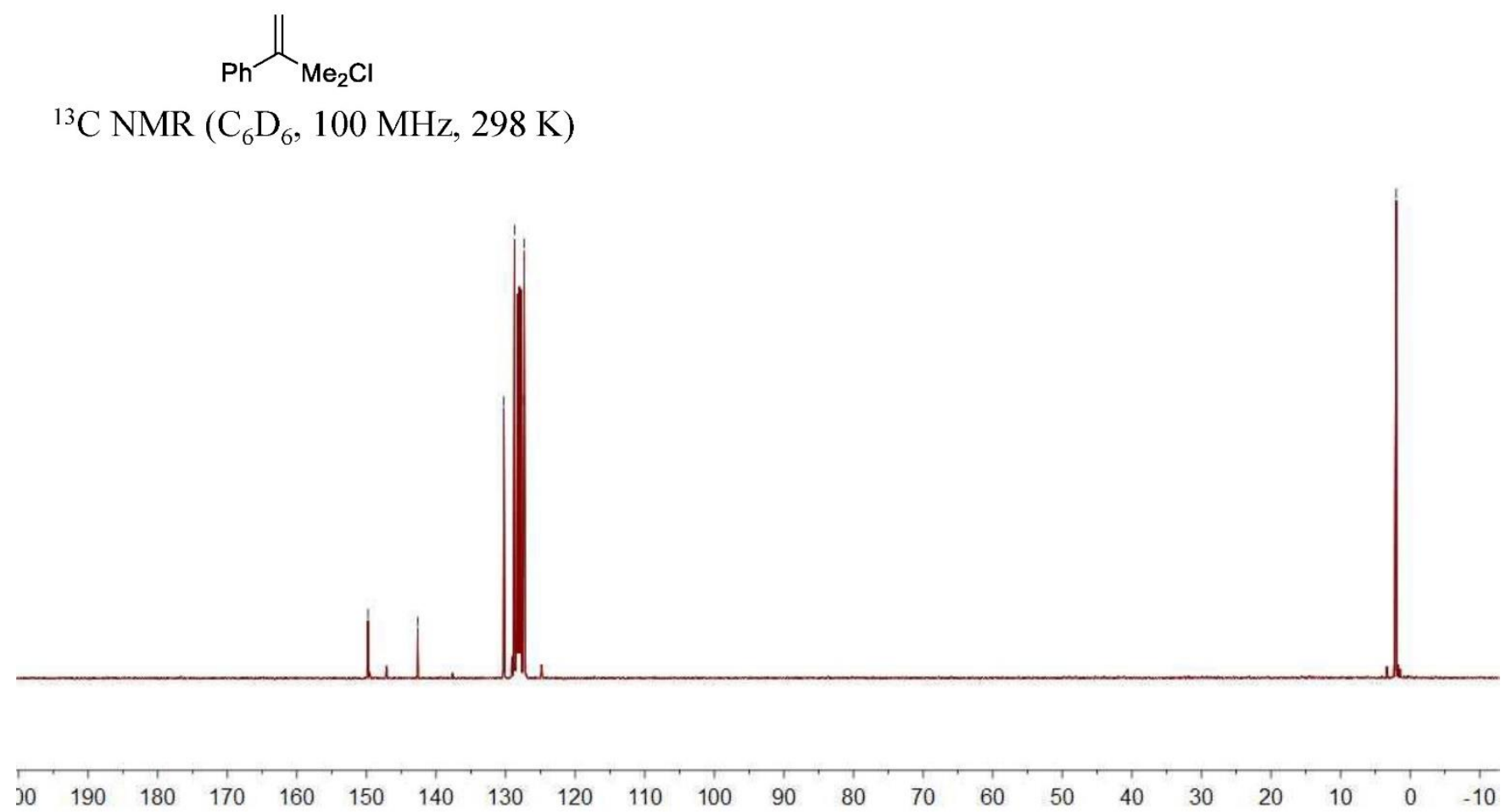

Figure S134. ${ }^{13} \mathrm{C}$ NMR spectrum of $\mathbf{P 5 3}$. 
${ }^{1} \mathrm{H} \mathrm{NMR}\left(\mathrm{CDCl}_{3}, 400 \mathrm{MHz}, 298 \mathrm{~K}\right)$
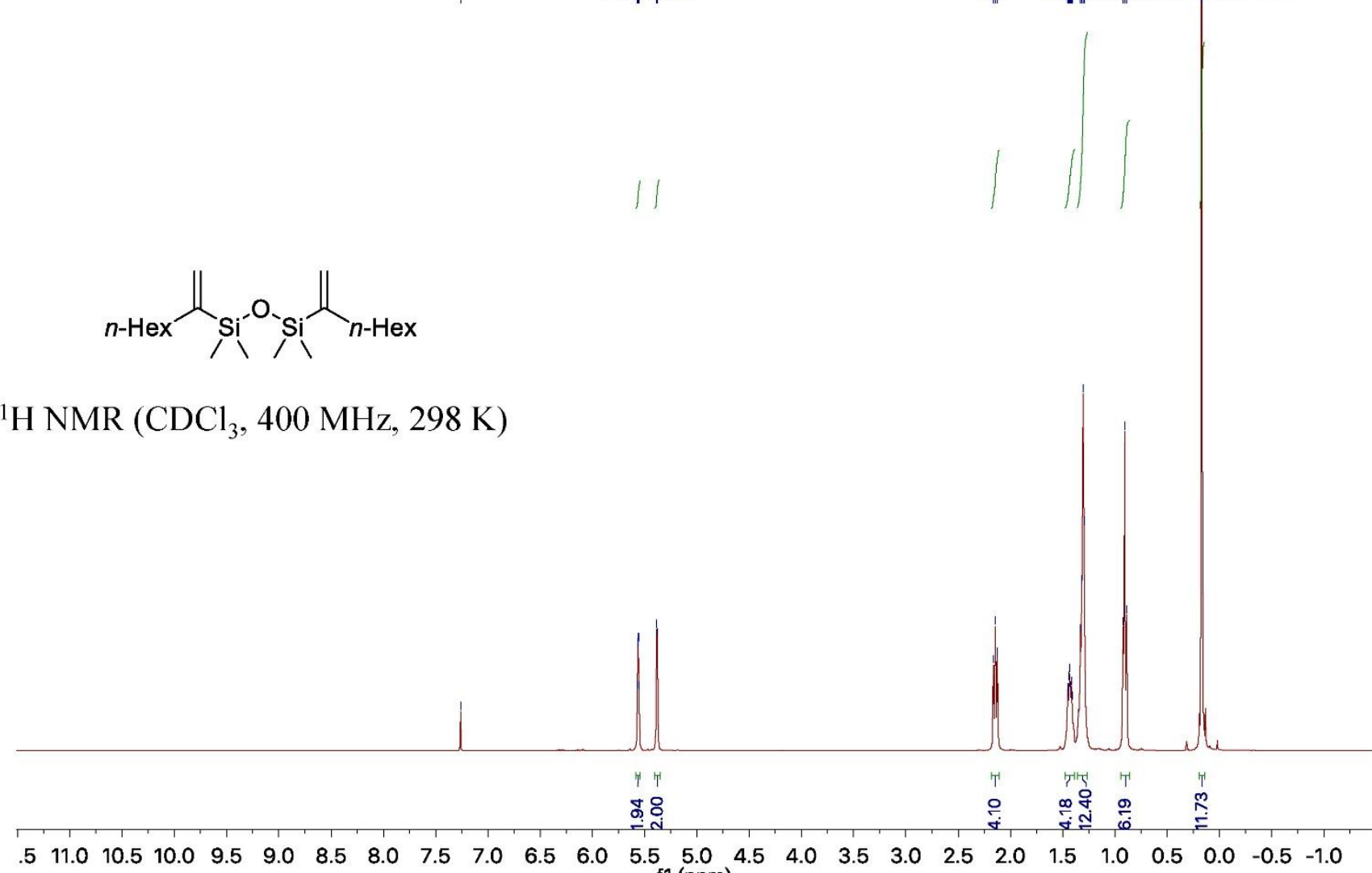

$\begin{array}{llllllllllllllllllllllllll}.5 & 11.0 & 10.5 & 10.0 & 9.5 & 9.0 & 8.5 & 8.0 & 7.5 & 7.0 & 6.5 & 6.0 & 5.5 & 5.0 & 4.5 & 4.0 & 3.5 & 3.0 & 2.5 & 2.0 & 1.5 & 1.0 & 0.5 & 0.0 & -0.5 & -1.0\end{array}$

Figure S135. ${ }^{1} \mathrm{H}$ NMR spectrum of P54. 


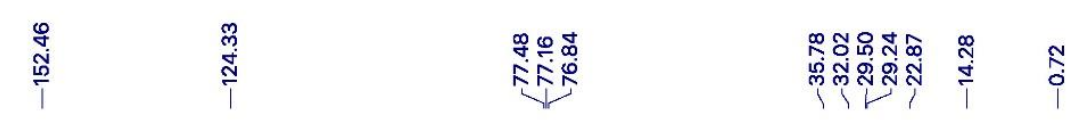

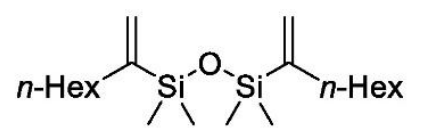

${ }^{13} \mathrm{C}$ NMR $\left(\mathrm{CDCl}_{3}, 100 \mathrm{MHz}, 298 \mathrm{~K}\right)$

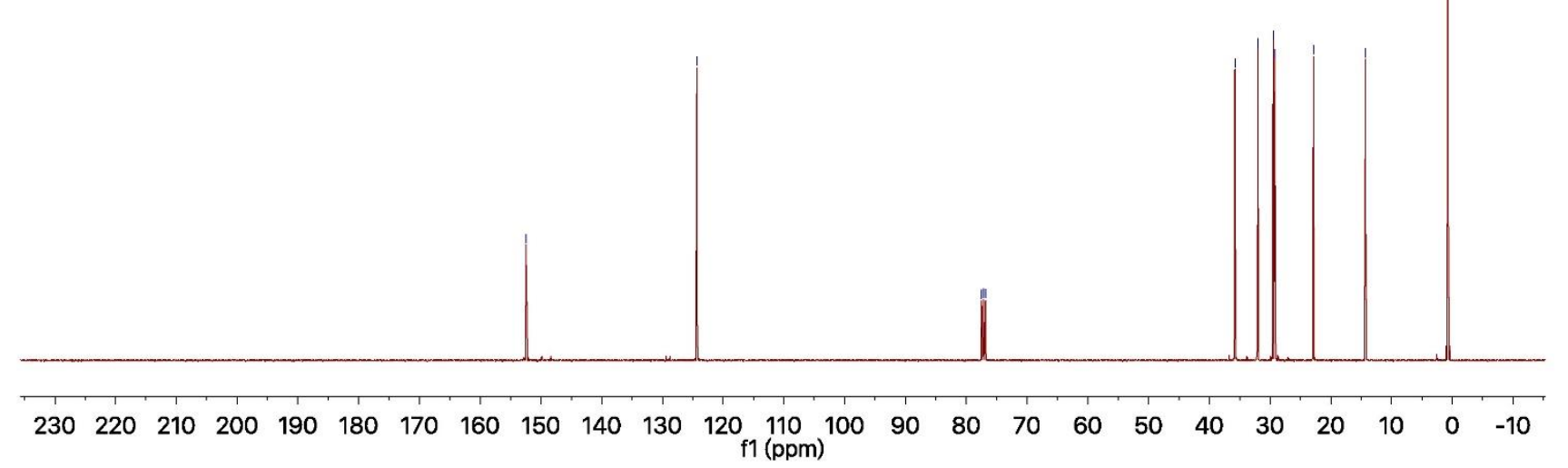

Figure S136. ${ }^{13} \mathrm{C}$ NMR spectrum of $\mathbf{P 5 4}$. 


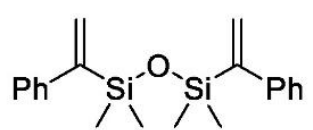

${ }^{1} \mathrm{H}$ NMR $\left(\mathrm{CDCl}_{3}, 400 \mathrm{MHz}, 294 \mathrm{~K}\right)$

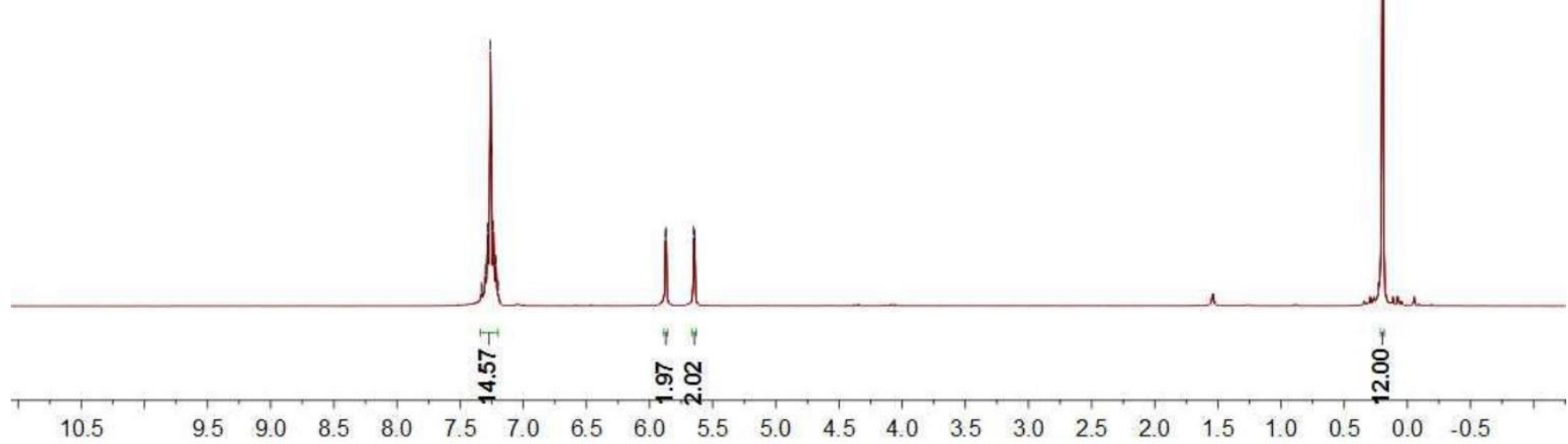

Figure S137. ${ }^{1} \mathrm{H}$ NMR spectrum of $\mathbf{P 5 5}$. 


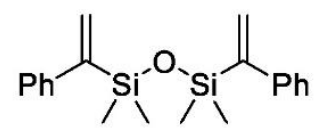

${ }^{13} \mathrm{C} \mathrm{NMR}\left(\mathrm{CDCl}_{3}, 100 \mathrm{MHz}, 298 \mathrm{~K}\right)$

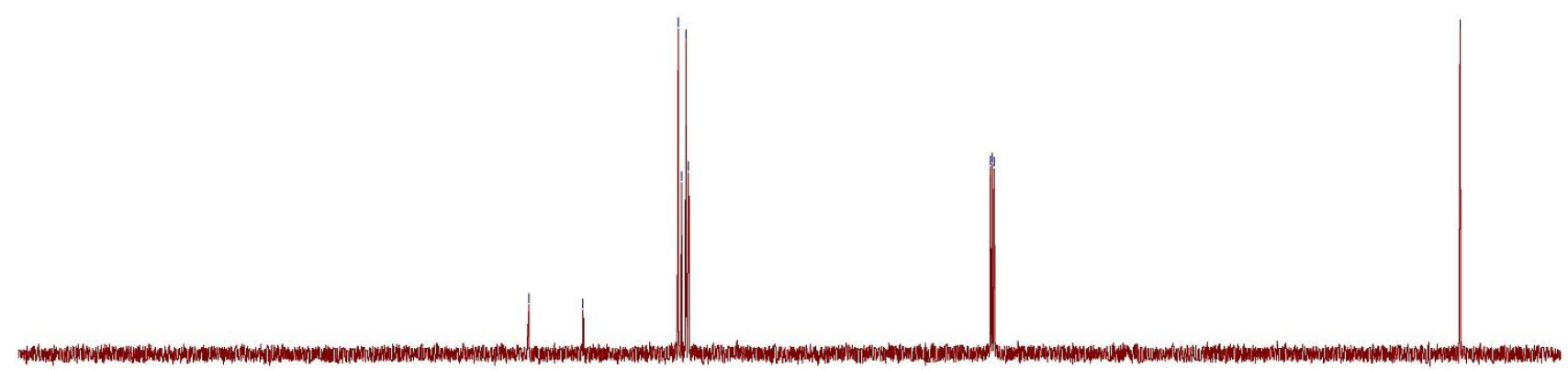

$\begin{array}{llllllllllllllllllllllllll}230 & 220 & 210 & 200 & 190 & 180 & 170 & 160 & 150 & 140 & 130 & 120 & 110 & 100 & 90 & 80 & 70 & 60 & 50 & 40 & 30 & 20 & 10 & 0 & -10\end{array}$

Figure S138. ${ }^{13} \mathrm{C}$ NMR spectrum of $\mathbf{P 5 5}$. 


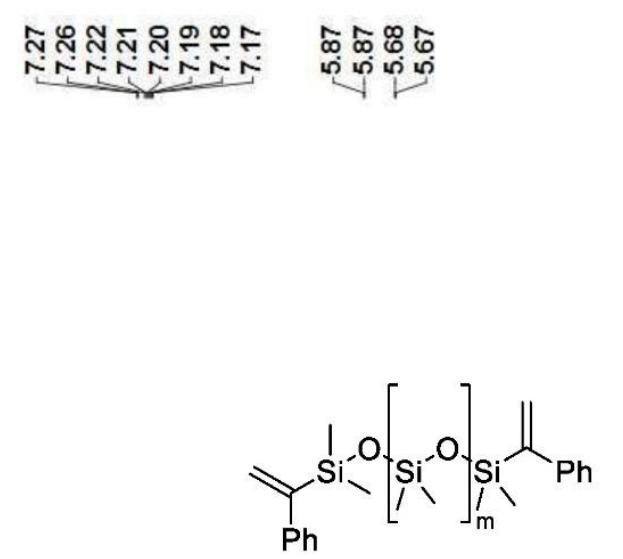

${ }^{1} \mathrm{H}$ NMR (400 MHz, $\mathrm{CDCl}_{3}, 294 \mathrm{~K}$ )

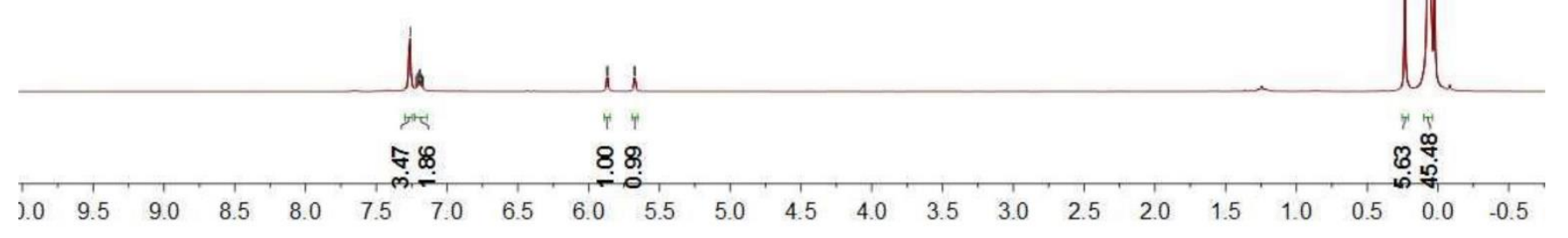

Figure S139. ${ }^{1} \mathrm{H}$ NMR spectrum of $\mathbf{P 5 6}$. 


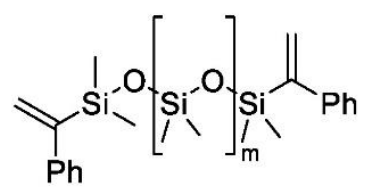

${ }^{13} \mathrm{C}$ NMR (100 MHz, $\mathrm{CDCl}_{3}, 294 \mathrm{~K}$ )
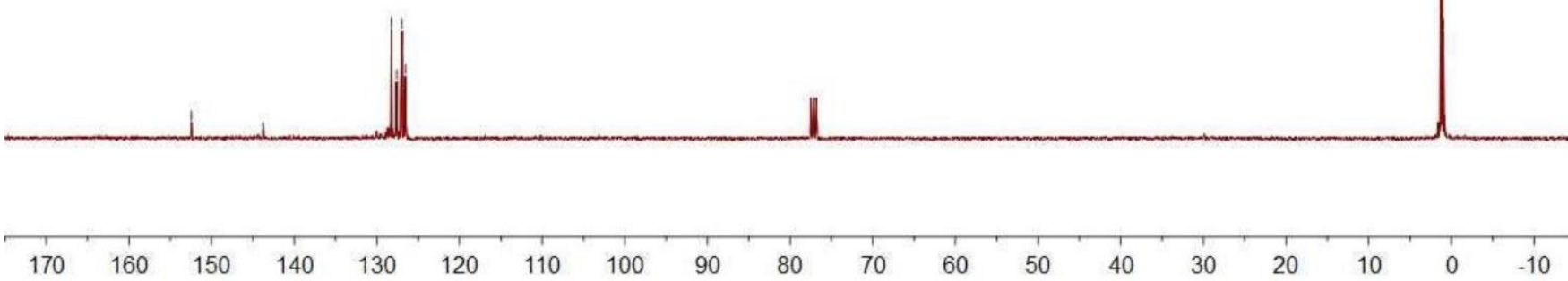

Figure S140. ${ }^{13} \mathrm{C}$ NMR spectrum of $\mathbf{P 5 6}$. 


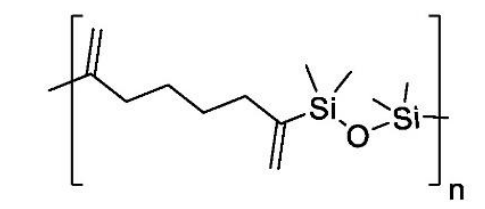

${ }^{1} \mathrm{H}$ NMR (400 MHz, $\mathrm{CDCl}_{3}, 298 \mathrm{~K}$ )

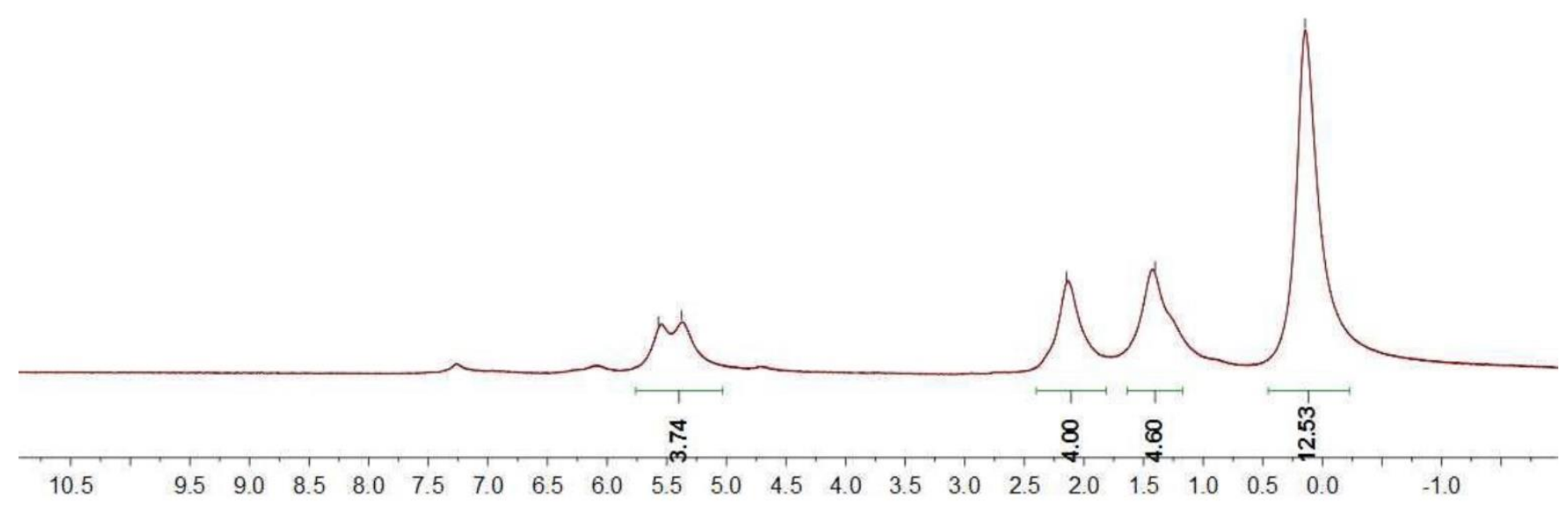

Figure S141. ${ }^{1} \mathrm{H}$ NMR spectrum of P57. 


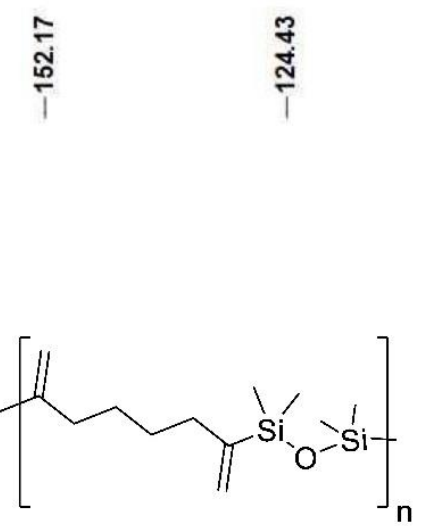

官

$\underset{i}{i}$

${ }^{13} \mathrm{C}$ NMR $\left(100 \mathrm{MHz}, \mathrm{CDCl}_{3}, 298 \mathrm{~K}\right)$

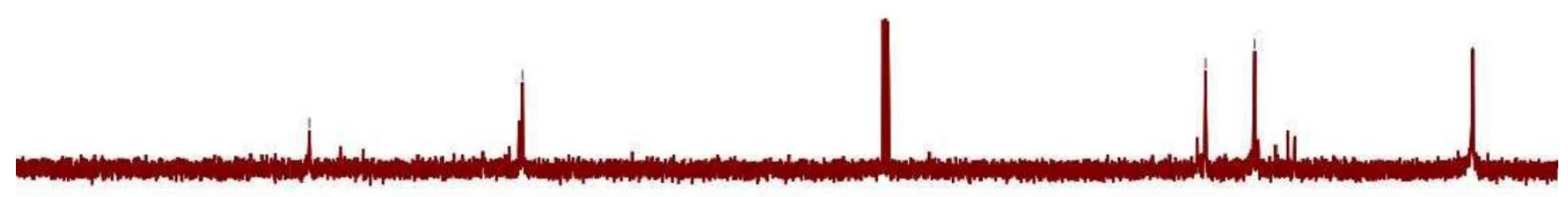

5

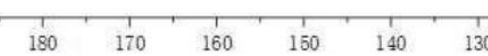

120

$\mathrm{f1} \stackrel{90}{\mathrm{gppm})}$

Figure S142. ${ }^{13} \mathrm{C}$ NMR spectrum of P57. 


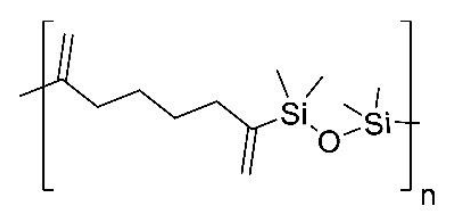

DEPT-135 (100 MHz, $\left.\mathrm{CDCl}_{3}, 298 \mathrm{~K}\right)$

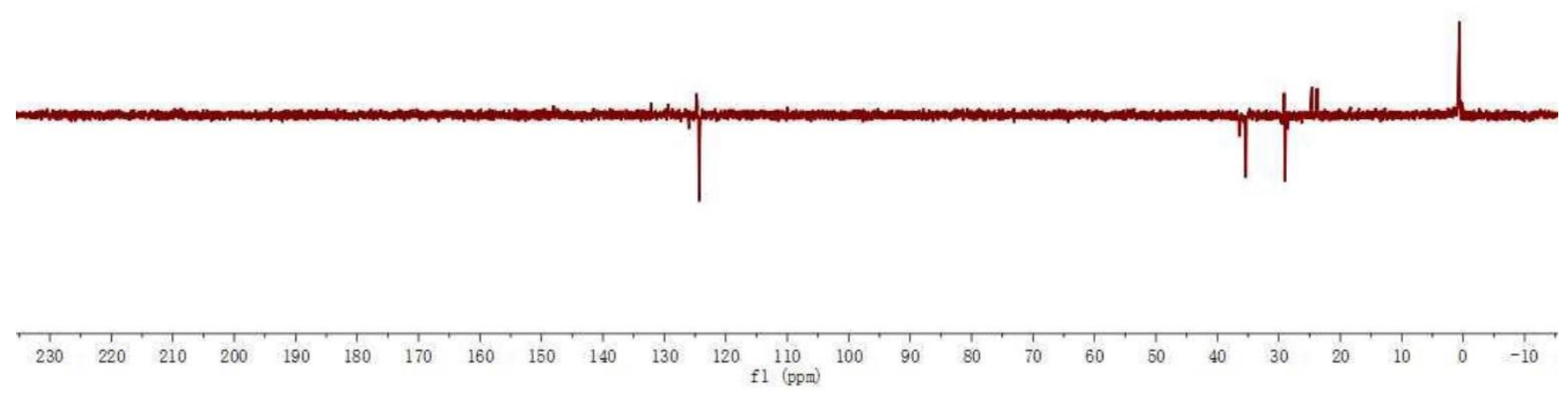

Figure S143. DEPT-135 of P57. 


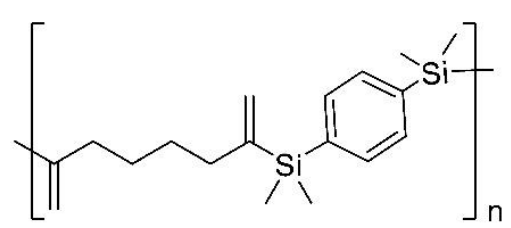

${ }^{1} \mathrm{H}$ NMR (400 MHz, $\mathrm{CDCl}_{3}, 303 \mathrm{~K}$ )

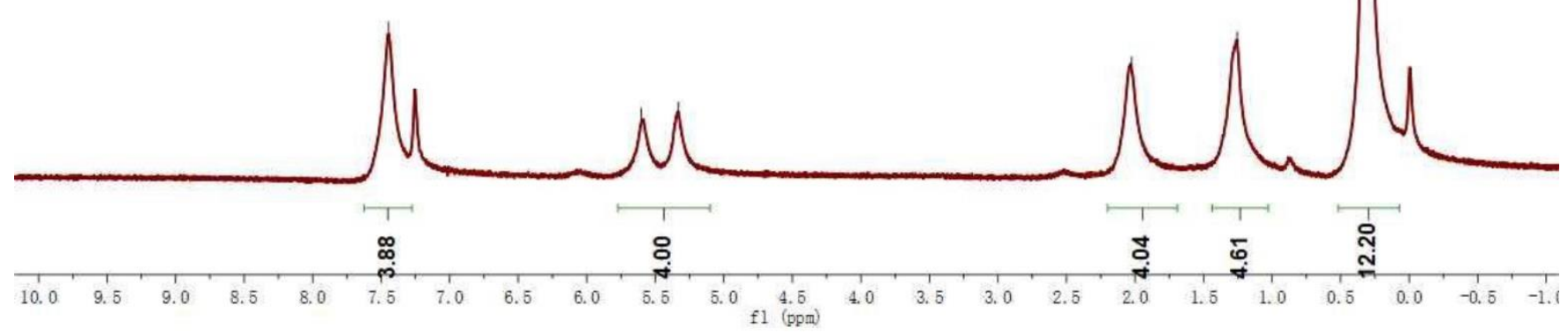

Figure S144. ${ }^{1} \mathrm{H}$ NMR spectrum of $\mathbf{P 5 8}$. 


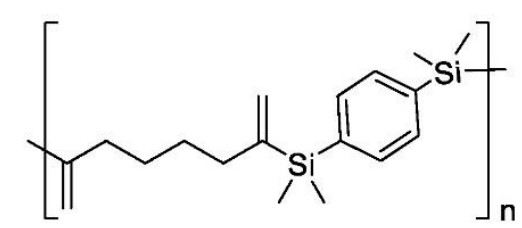

${ }^{13} \mathrm{C}$ NMR (100 MHz, $\left.\mathrm{CDCl}_{3}, 303 \mathrm{~K}\right)$
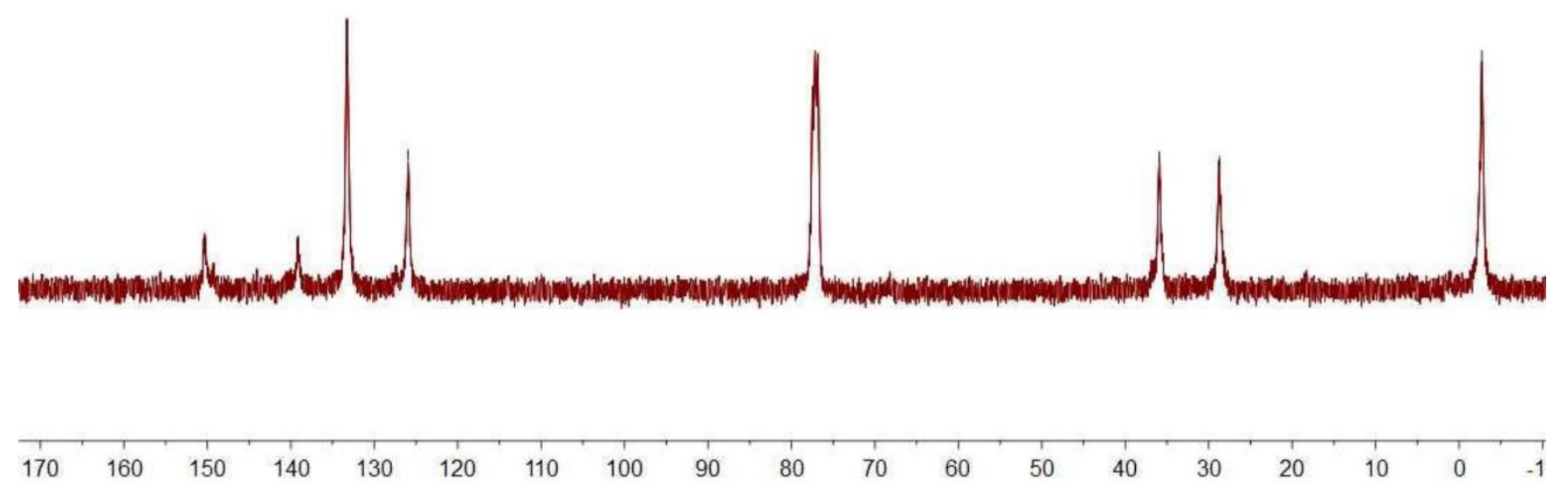

Figure S145. ${ }^{13} \mathrm{C}$ NMR spectrum of P58. 


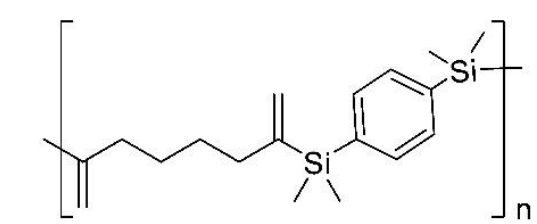

DEPT- $135^{\circ}\left(100 \mathrm{MHz}, \mathrm{CDCl}_{3}, 303 \mathrm{~K}\right)$

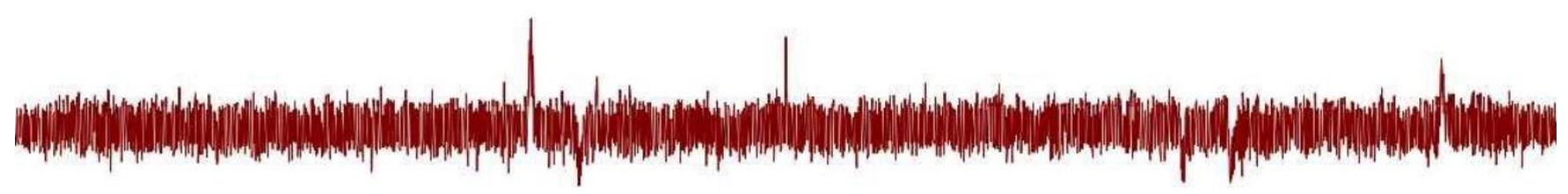

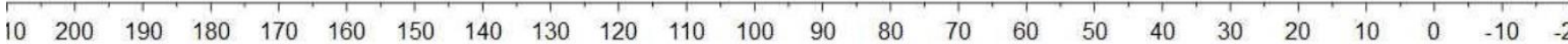

Figure S146. DEPT-135 of P58. 


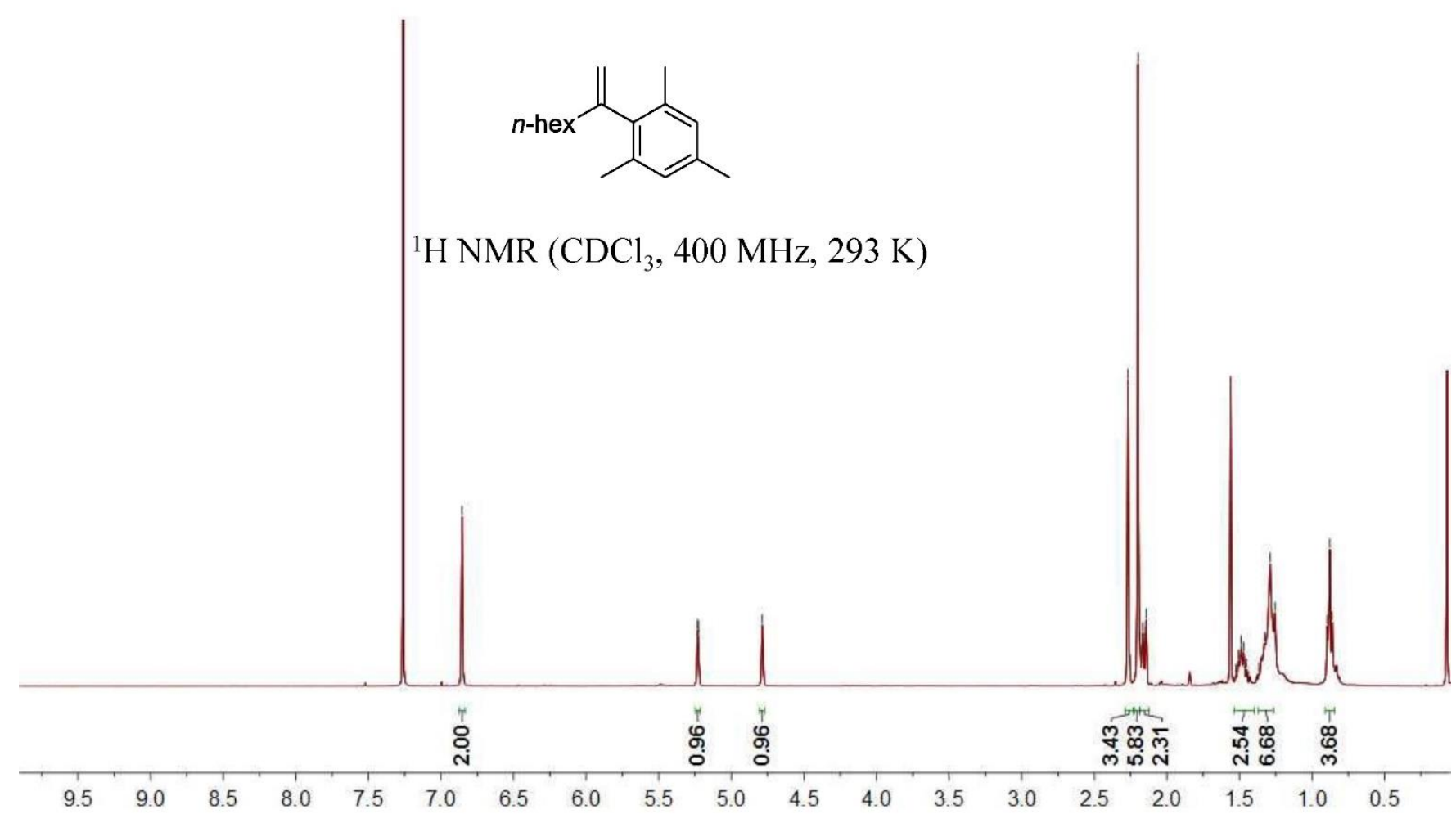

Figure S147. ${ }^{1} \mathrm{H}$ NMR spectrum of P59. 


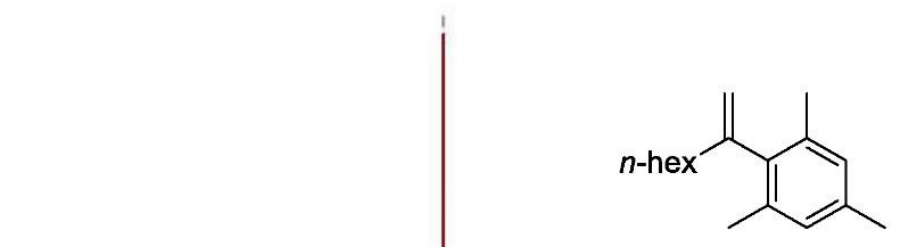

${ }^{13} \mathrm{C} \mathrm{NMR}\left(\mathrm{CDCl}_{3}, 100 \mathrm{MHz}, 293 \mathrm{~K}\right)$

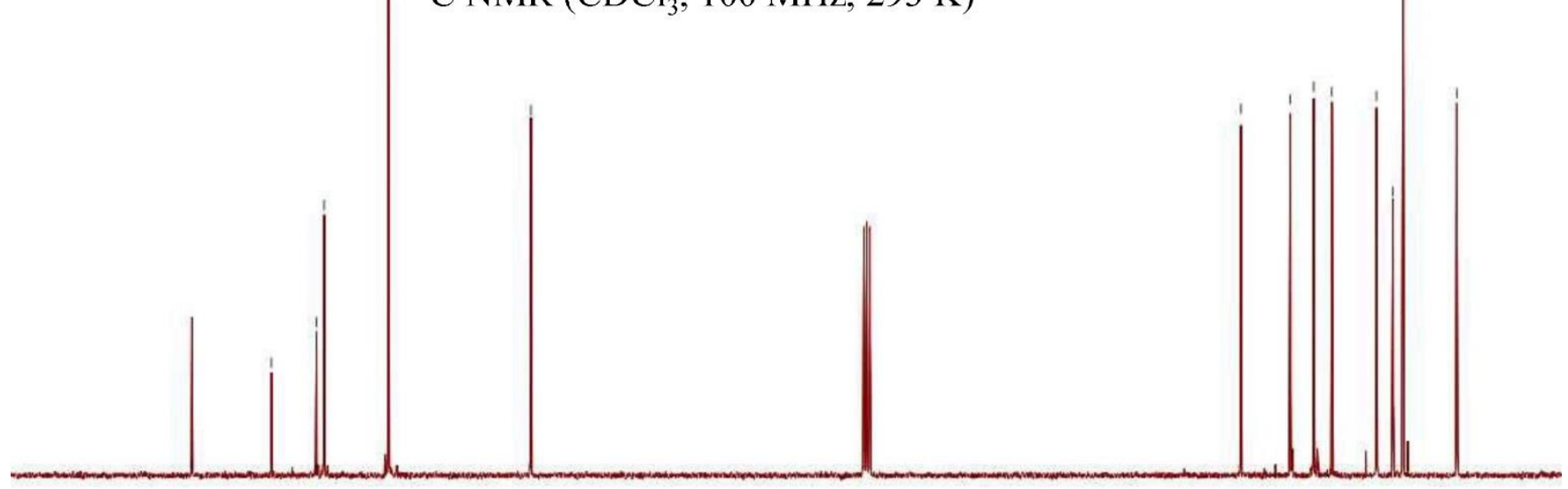

Figure S148. ${ }^{13} \mathrm{C}$ NMR spectrum of P59. 


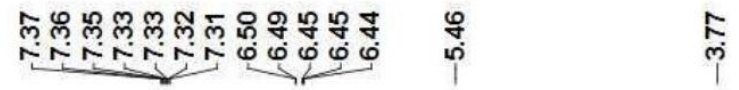

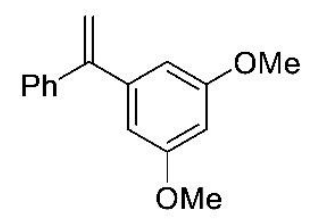

'H NMR ( $\left.\mathrm{CDCl}_{3}, 400 \mathrm{MHz}, 294 \mathrm{~K}\right)$

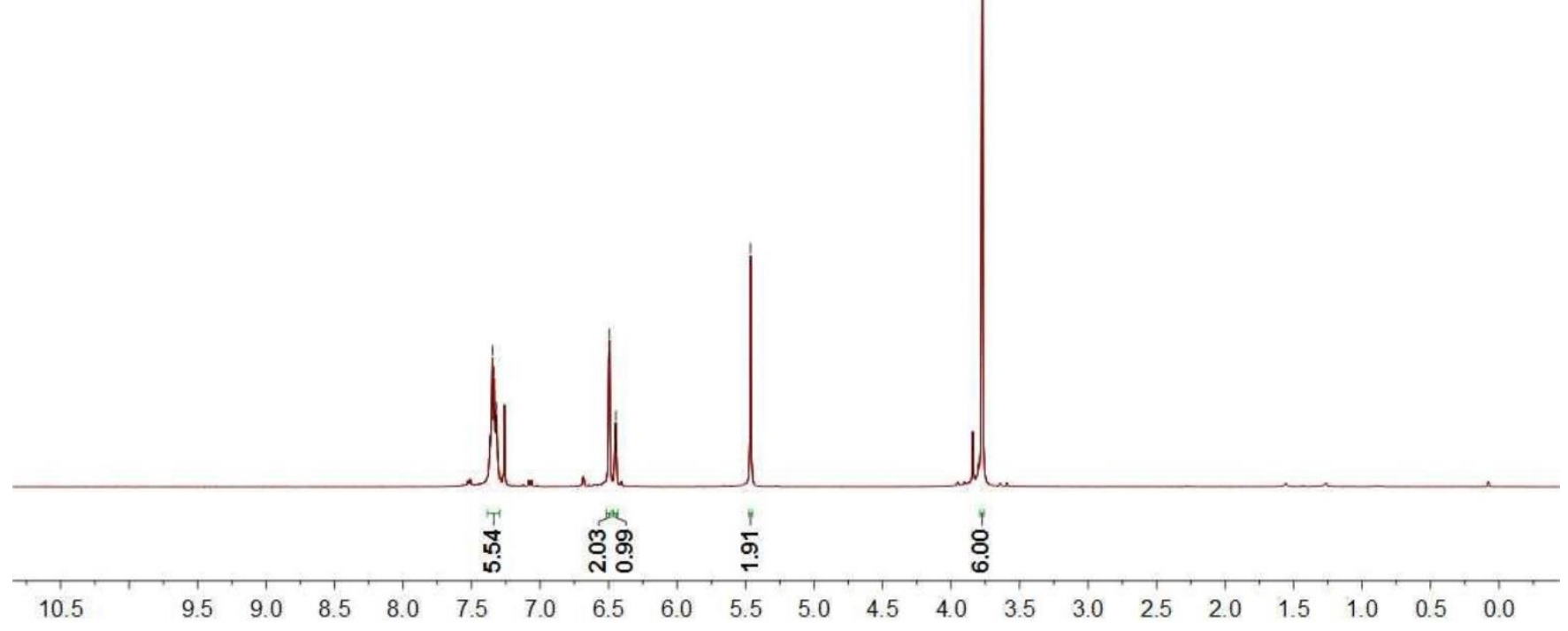

Figure S149. ${ }^{1} \mathrm{H}$ NMR spectrum of $\mathbf{P 6 0}$. 

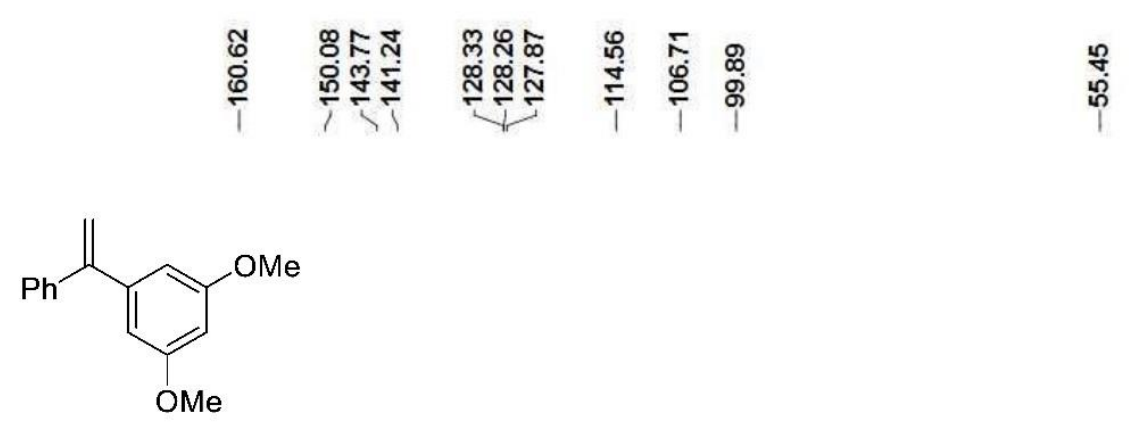

${ }^{13} \mathrm{C} \mathrm{NMR}\left(\mathrm{CDCl}_{3}, 100 \mathrm{MHz}, 294 \mathrm{~K}\right)$
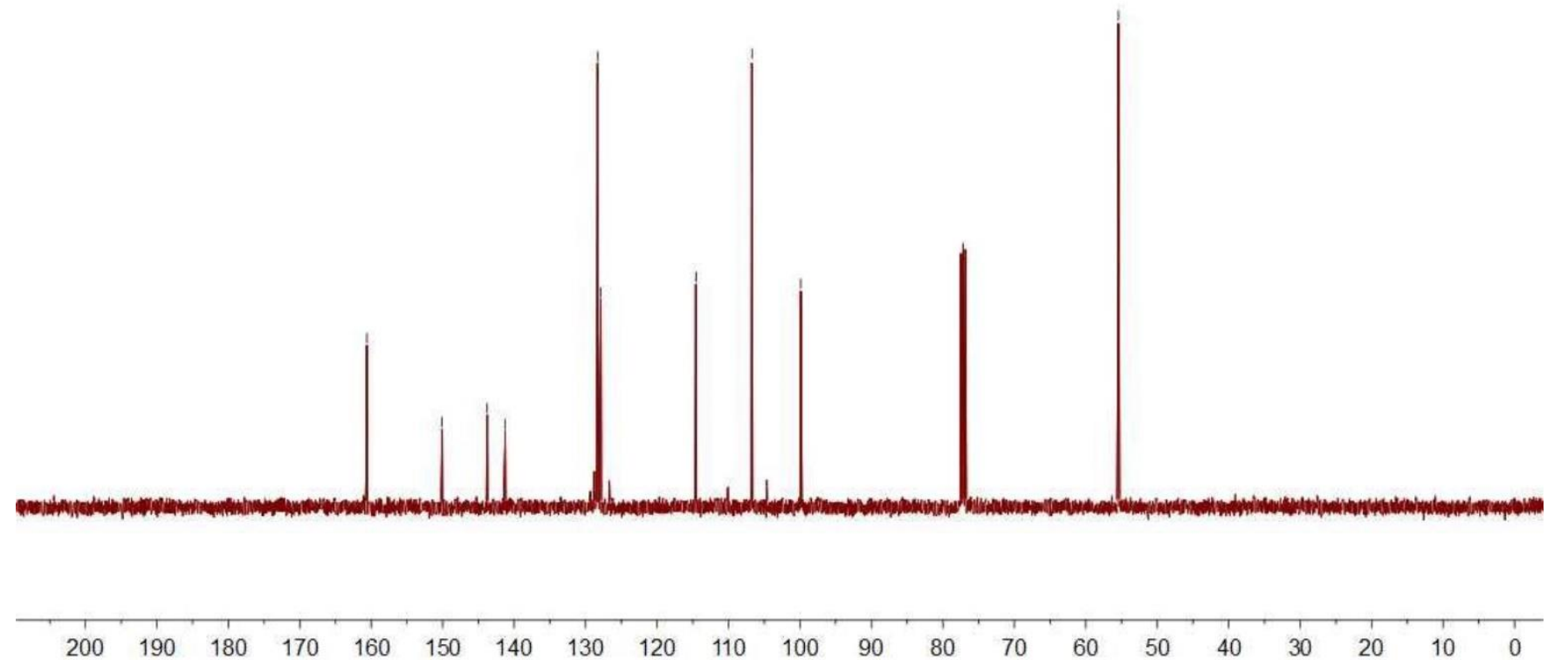

Figure S150. ${ }^{13} \mathrm{C}$ NMR spectrum of $\mathbf{P 6 0}$. 


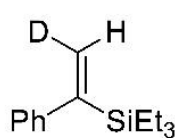

'H NMR (400 MHz, CDCl $3,303 \mathrm{~K}$ )

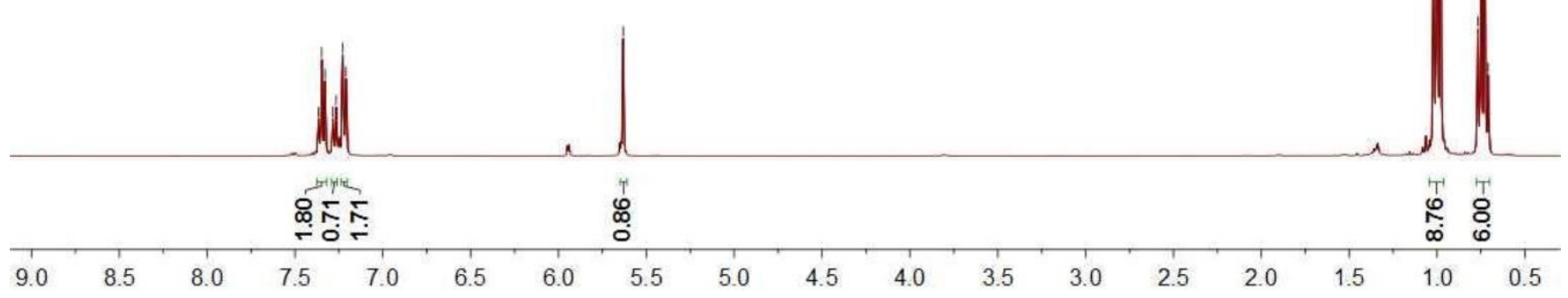

Figure S151. ${ }^{1} \mathrm{H}$ NMR spectrum of (E)-triethyl(1-phenylvinyl-2-d)silane. 


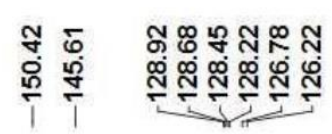

$$
\overbrace{\mathrm{SiEt}_{3}}^{\mathrm{H}}
$$

${ }^{13} \mathrm{C}$ NMR (100 MHz, $\left.\mathrm{CDCl}_{3}, 303 \mathrm{~K}\right)$

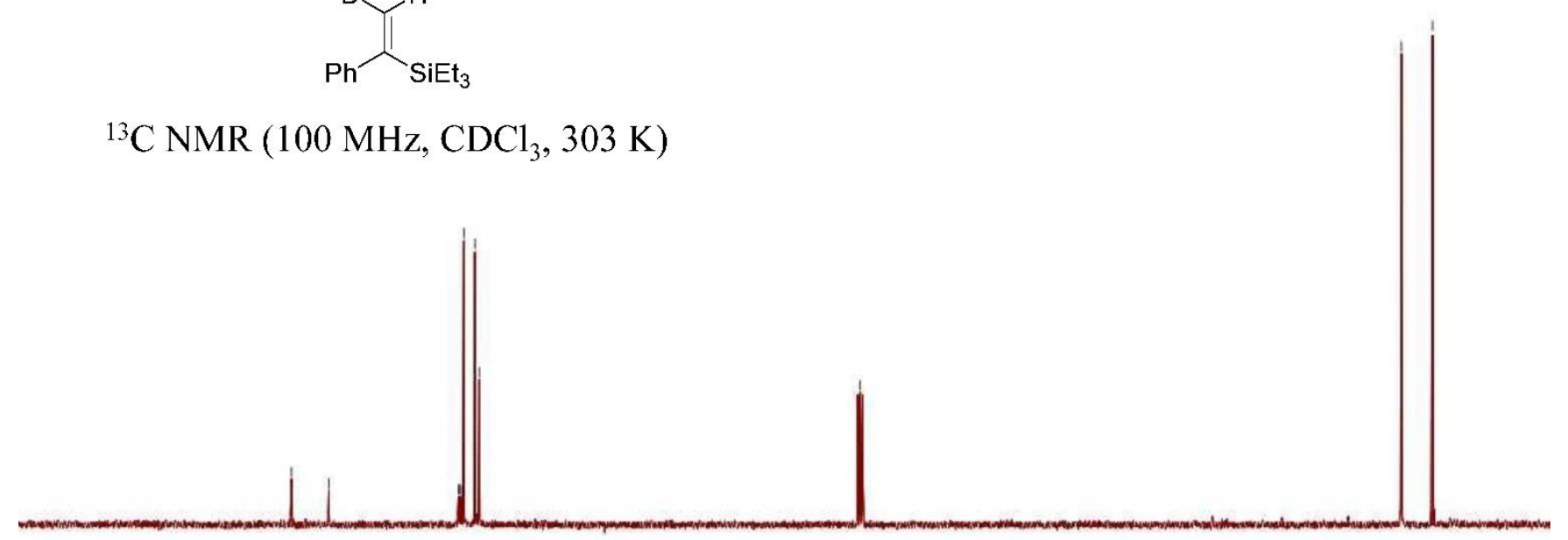

180

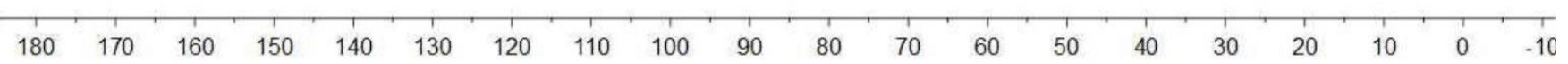

Figure S152. ${ }^{13} \mathrm{C}$ NMR spectrum of (E)-triethyl(1-phenylvinyl-2- $d$ ) silane. 


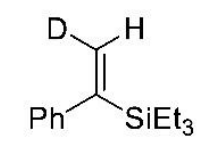

${ }^{2} \mathrm{H}$ NMR (61 MHz, $\left.\mathrm{CDCl}_{3}, 296 \mathrm{~K}\right)$

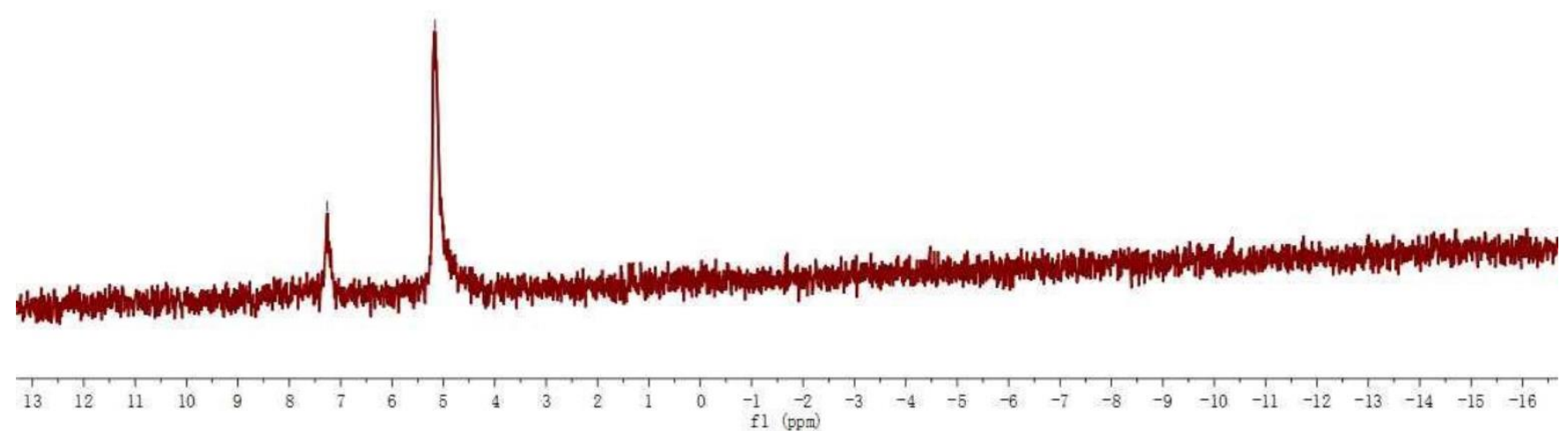

Figure S153. ${ }^{2} \mathrm{H}$ NMR spectrum of (E)-triethyl(1-phenylvinyl-2-d)silane. 


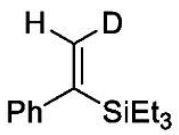

${ }^{1} \mathrm{H}$ NMR (400 MHz, $\mathrm{CDCl}_{3}, 298 \mathrm{~K}$ )

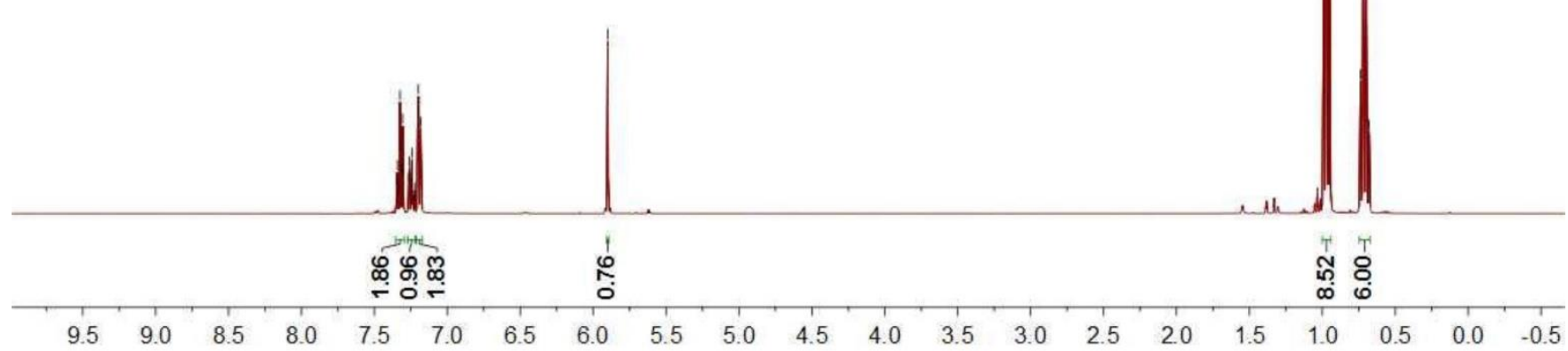

Figure S154. ${ }^{1} \mathrm{H}$ NMR spectrum of (Z)-triethyl(1-phenylvinyl-2-d)silane. 


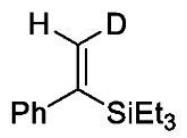

${ }^{13} \mathrm{C}$ NMR (100 MHz, $\left.\mathrm{CDCl}_{3}, 303 \mathrm{~K}\right)$

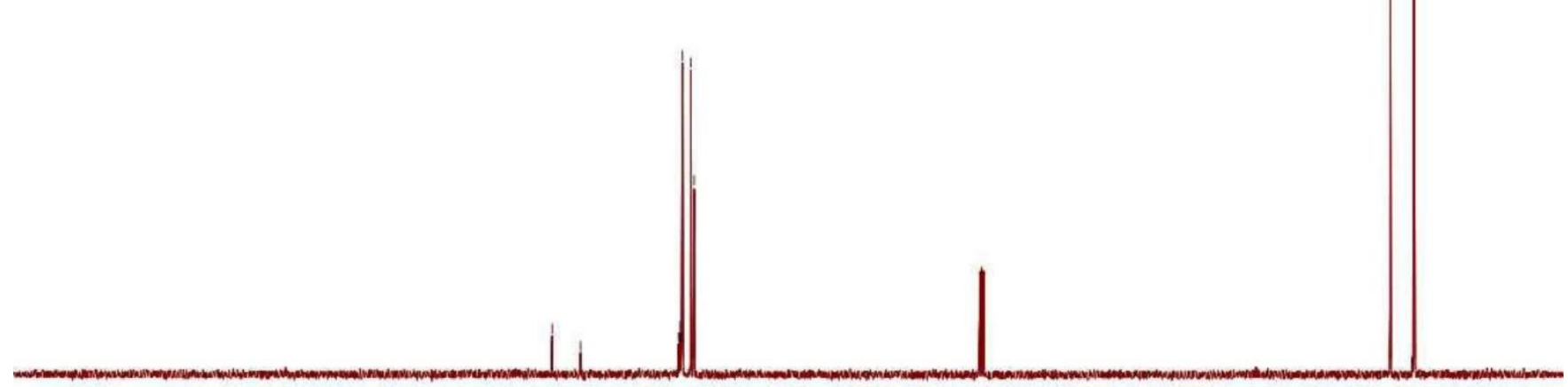

Figure S155. ${ }^{13} \mathrm{C}$ NMR spectrum of (Z)-triethyl(1-phenylvinyl-2- $d$ )silane. 


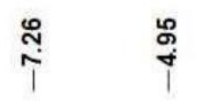

${ }_{\mathrm{Ph}}^{\mathrm{H}} \mathrm{I}_{\mathrm{SiEt}_{3}}^{\mathrm{D}}$

${ }^{2} \mathrm{H} \mathrm{NMR}\left(61 \mathrm{MHz}, \mathrm{CDCl}_{3}, 296 \mathrm{~K}\right)$
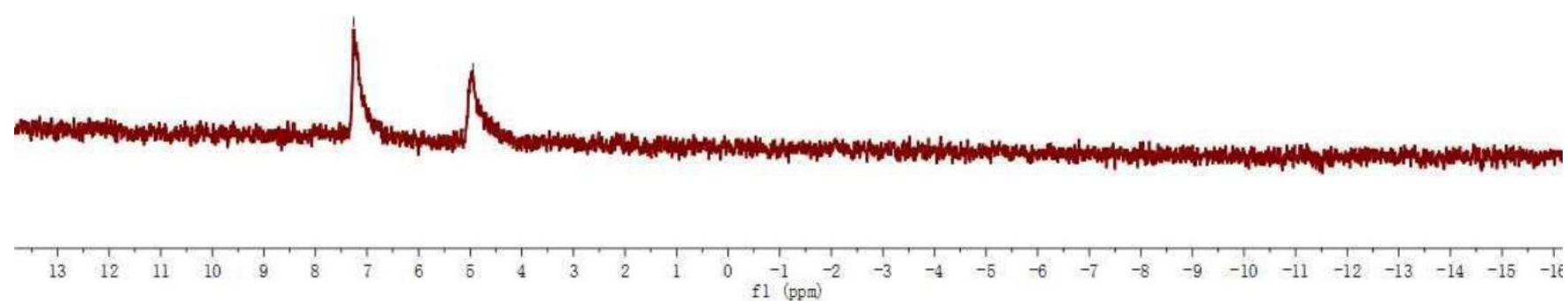

Figure S156. ${ }^{2} \mathrm{H}$ NMR spectrum of (Z)-triethyl(1-phenylvinyl-2-d)silane. 


$$
\mathrm{Ph} \equiv+\mathrm{Et}_{3} \mathrm{Si}-\mathrm{H} \frac{\text { Cat. 1 }}{\mathrm{THF,60^{ \circ } \mathrm { C } , 5 \mathrm { h }}} \underset{\alpha}{\mathrm{Ph}} \|_{\mathrm{SiEt}_{3}}+\underset{\mathrm{Ph}^{-(E)}}{\mathrm{SiEt}_{3}}+\int_{\substack{\mathrm{Ph} \\ \beta-(\mathrm{Z})}}^{\mathrm{SiEt}_{3}}
$$

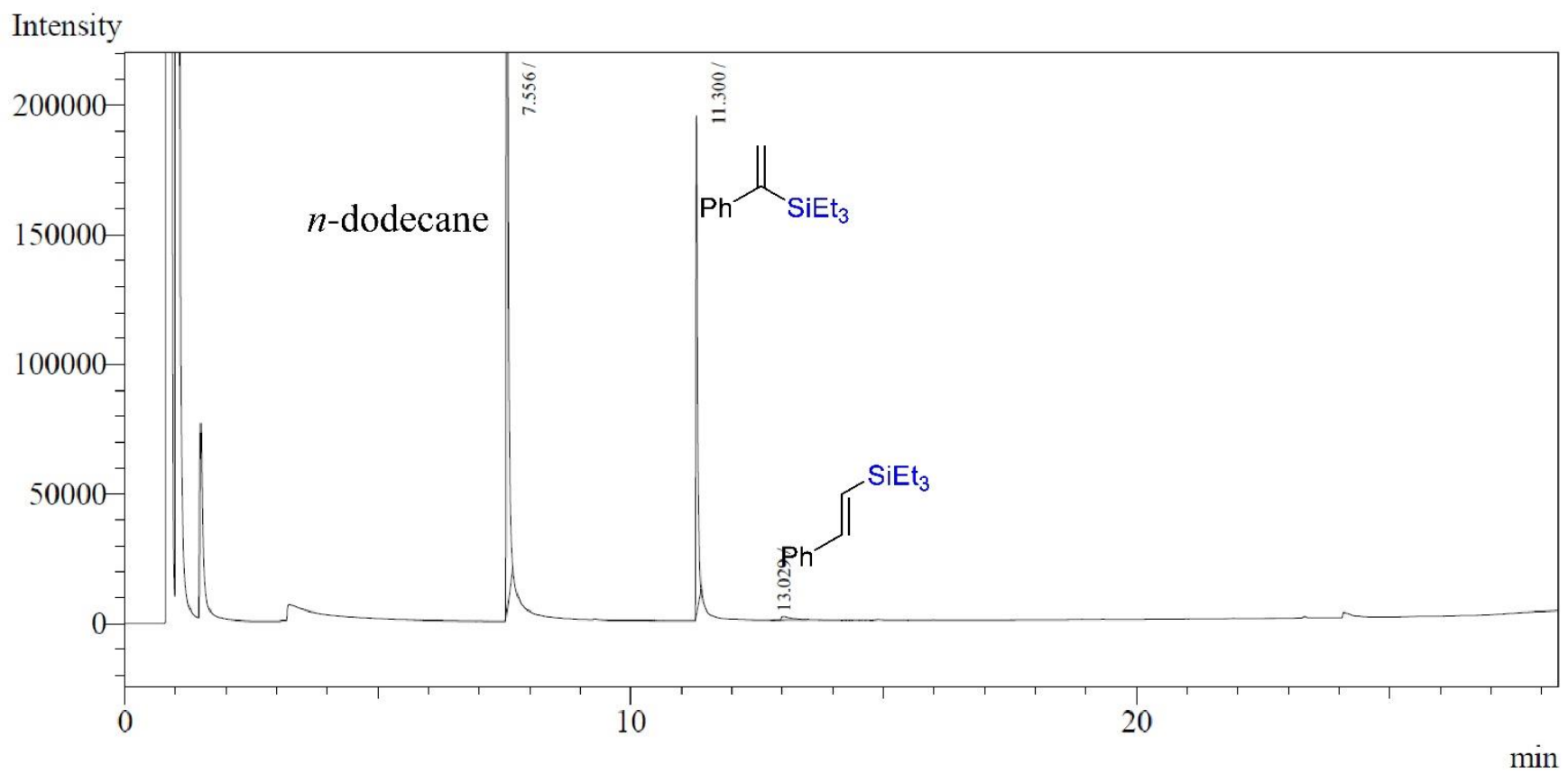

Figure S157. GC graph of the reaction of phenyl acetylene with triethylsilane catalyzed by $\mathbf{1}$. 


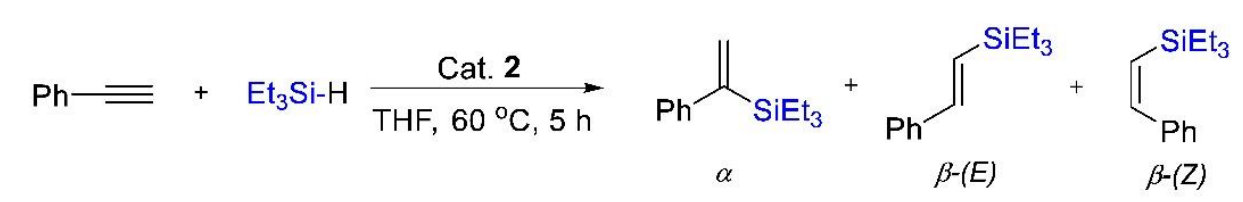

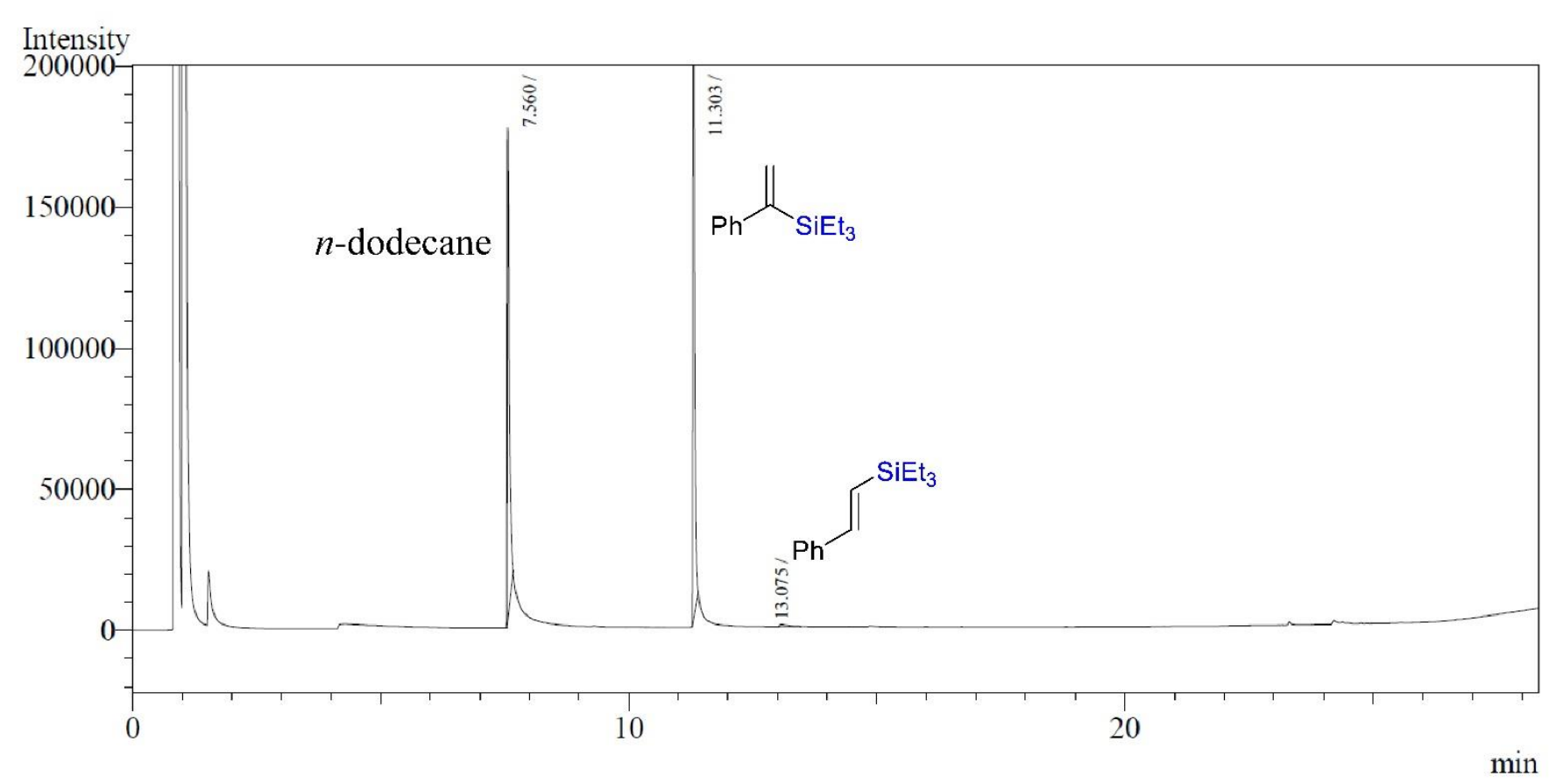

Figure S158. GC graph of the reaction of phenyl acetylene with triethylsilane catalyzed by 2 . 


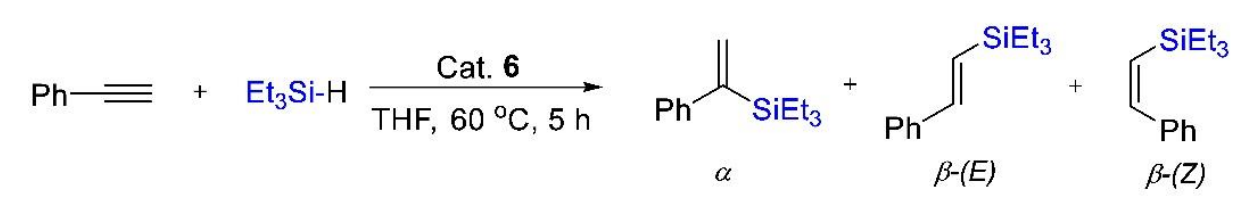

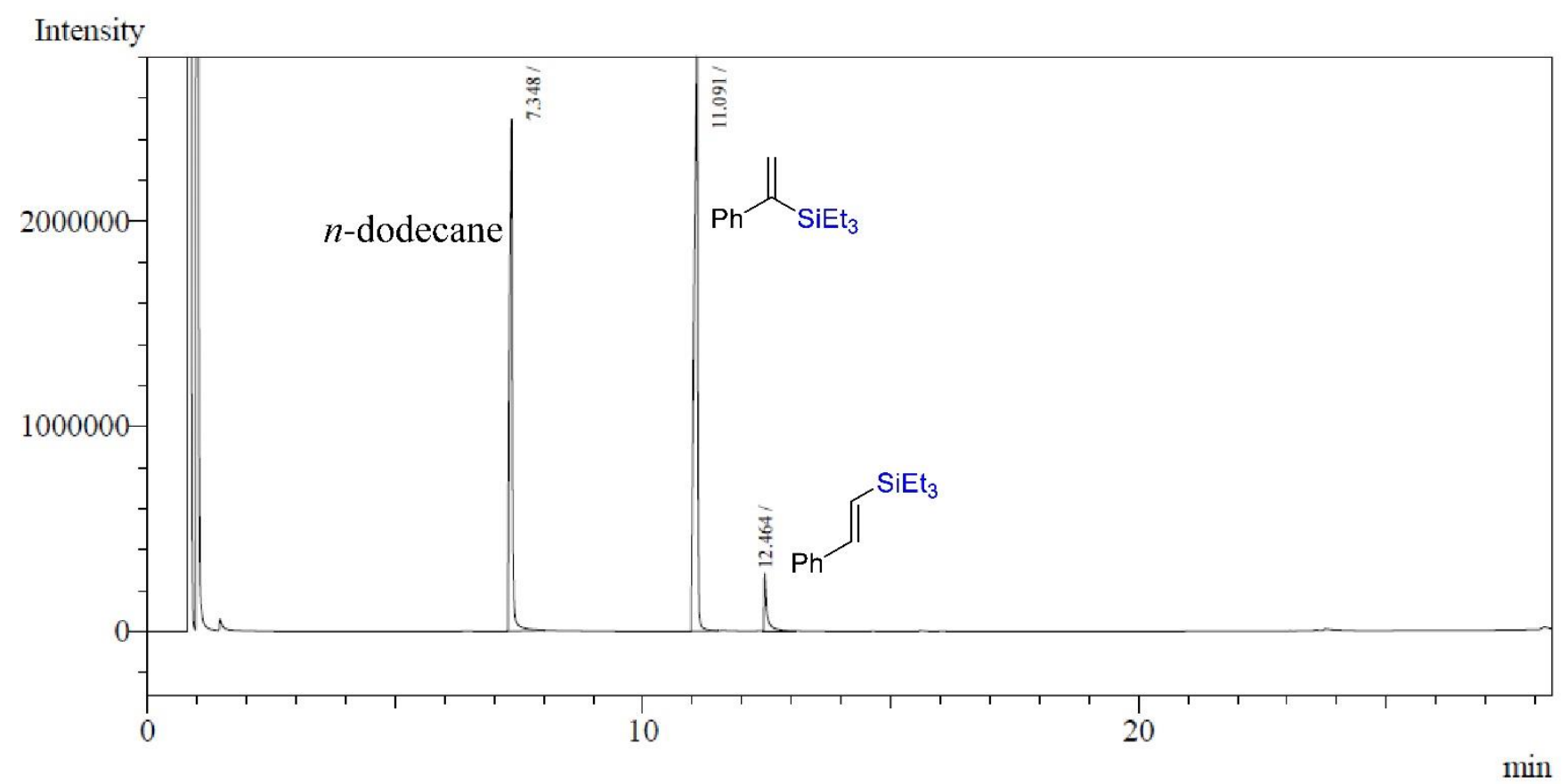

Figure S159. GC graph of the reaction of phenyl acetylene with triethylsilane catalyzed by 6 . 


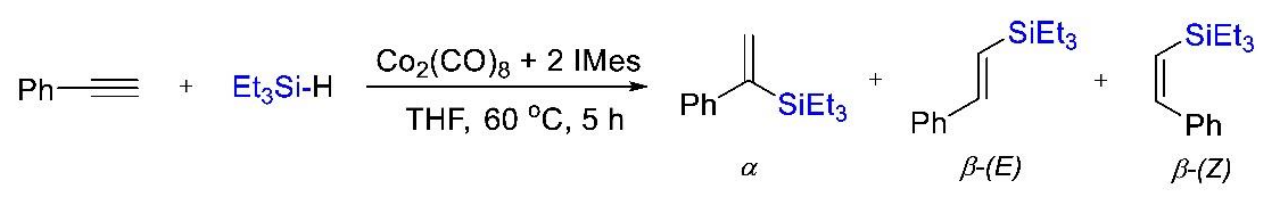

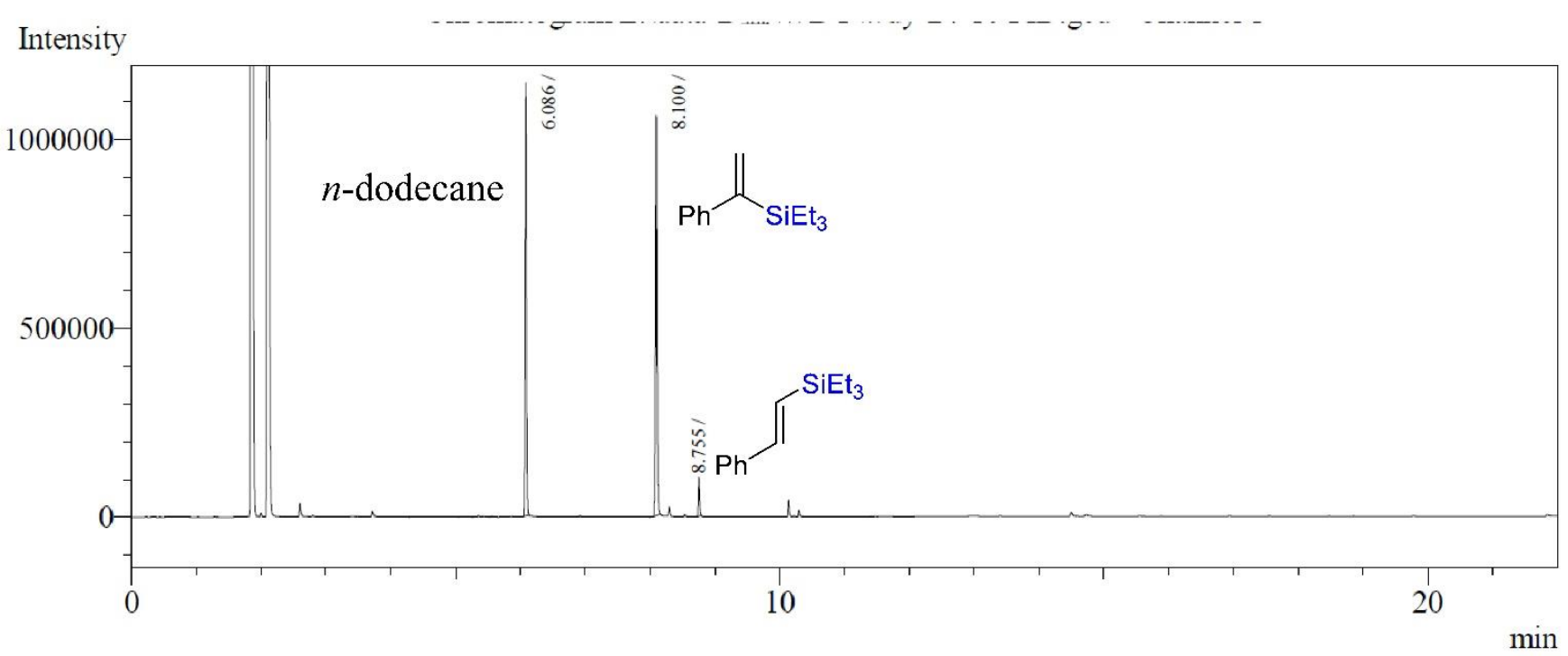

Figure S160. GC graph of the reaction of phenyl acetylene with triethylsilane catalyzed by $\mathrm{Co}_{2}(\mathrm{CO})_{8}$ and IMes. 


$$
\mathrm{Ph}=+\mathrm{Et}_{3} \mathrm{Si}-\mathrm{H} \underset{\mathrm{THF}, 60^{\circ} \mathrm{C}, 5 \mathrm{~h}}{\frac{\mathrm{Co}_{2}(\mathrm{CO})_{8}+2 \mathrm{PPh}_{3}}{\mathrm{Ph}}} \underset{\alpha}{\mathrm{SiEt}_{3}}+\underset{\mathrm{Ph}}{\int_{\beta-(E)}}+\underset{\beta-(\mathrm{Z})}{\mathrm{SiEt}_{3}}
$$

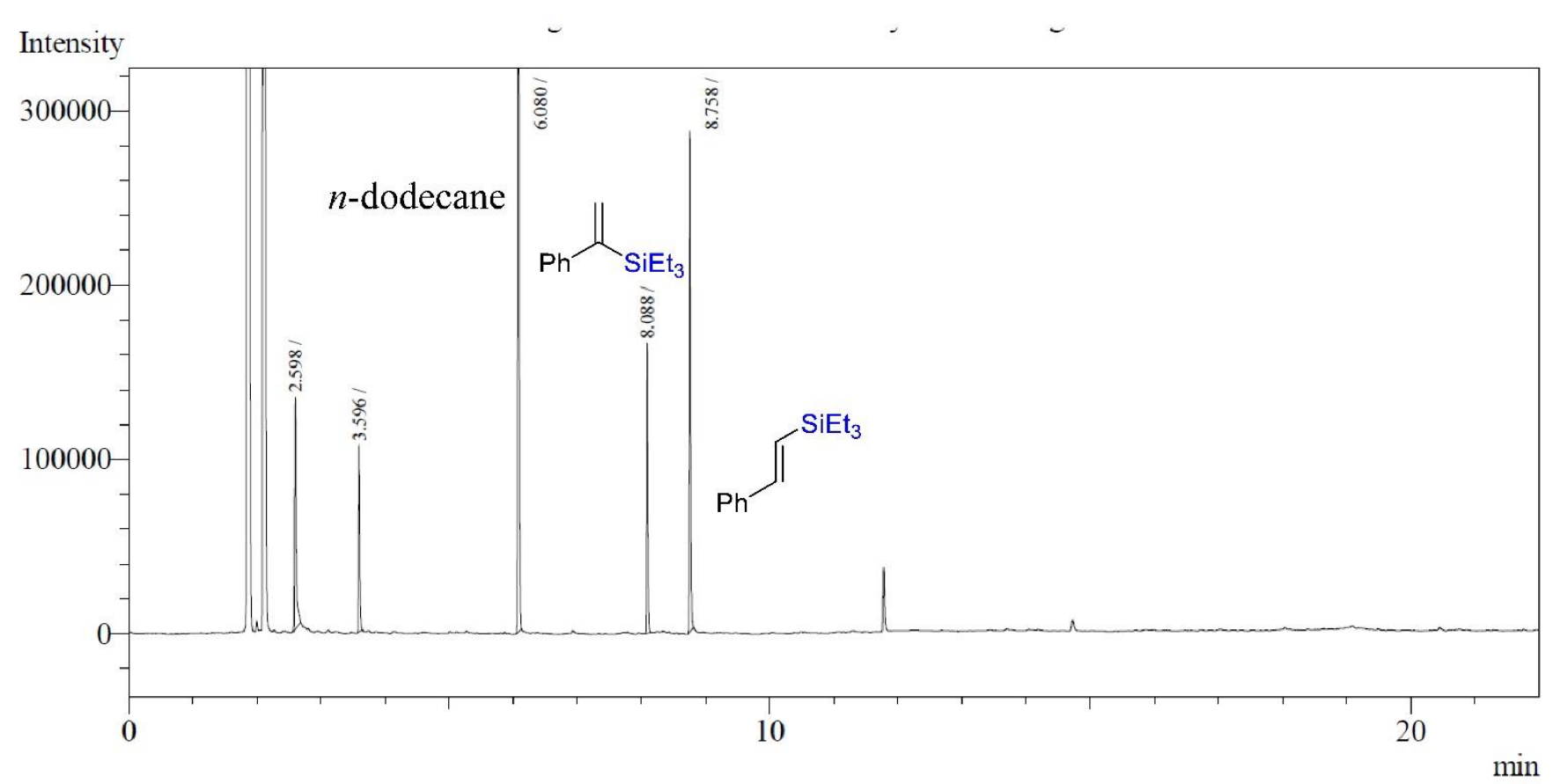

Figure S161. GC graph of the reaction of phenyl acetylene with triethylsilane catalyzed by $\mathrm{Co}_{2}(\mathrm{CO})_{8}$ and $\mathrm{PPh}_{3}$. 


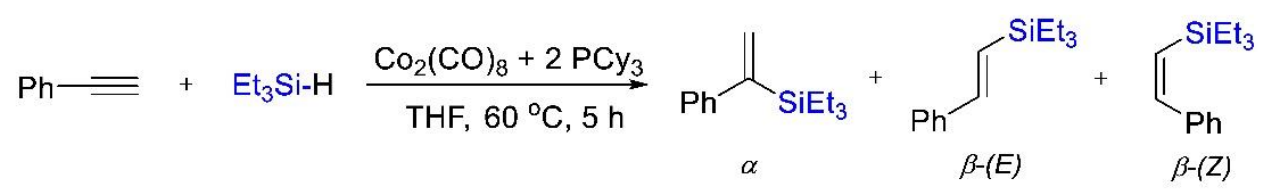

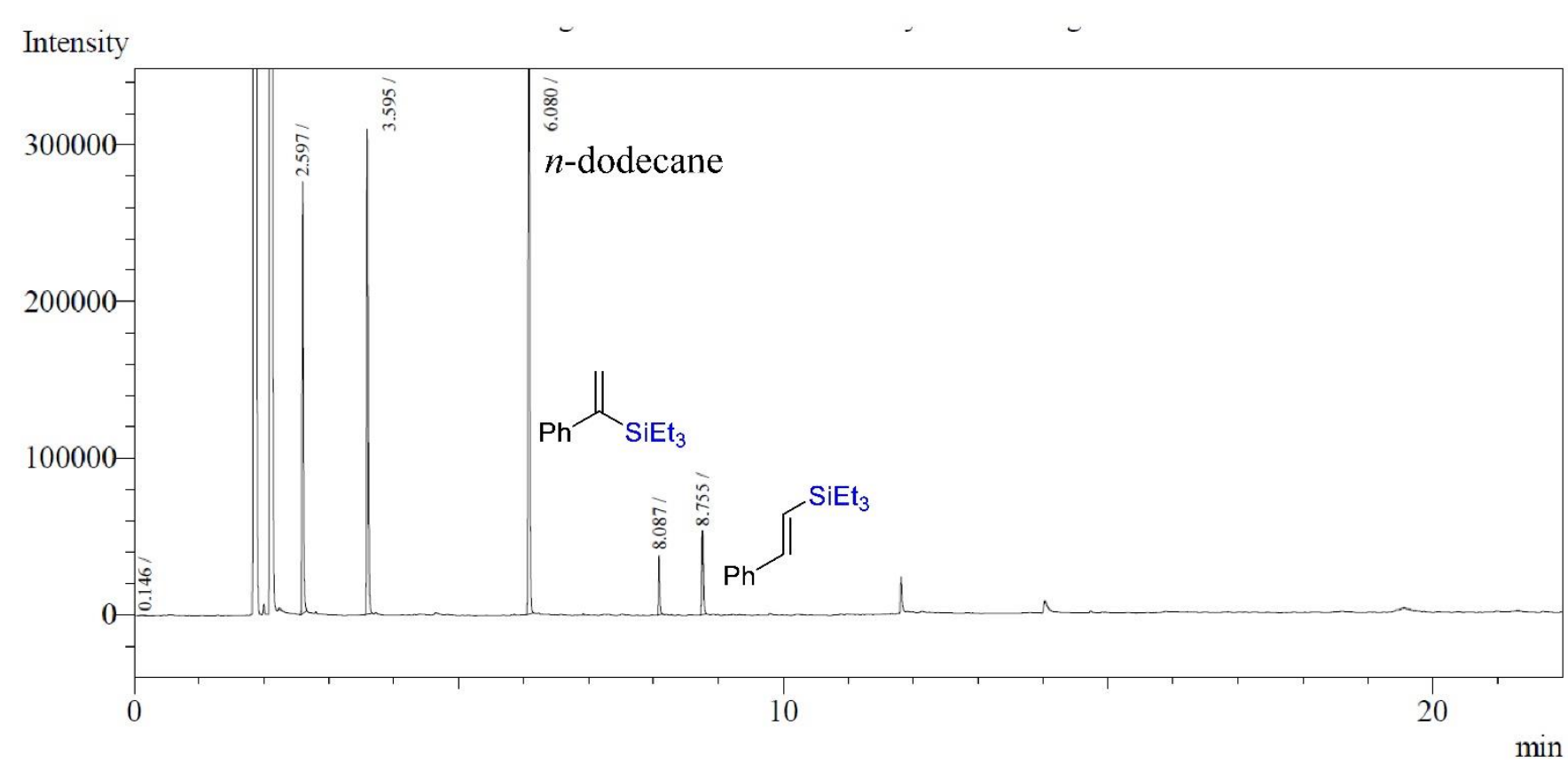

Figure S162. GC graph of the reaction of phenyl acetylene with triethylsilane catalyzed by $\mathrm{Co}_{2}(\mathrm{CO})_{8}$ and $\mathrm{PCy}_{3}$. 


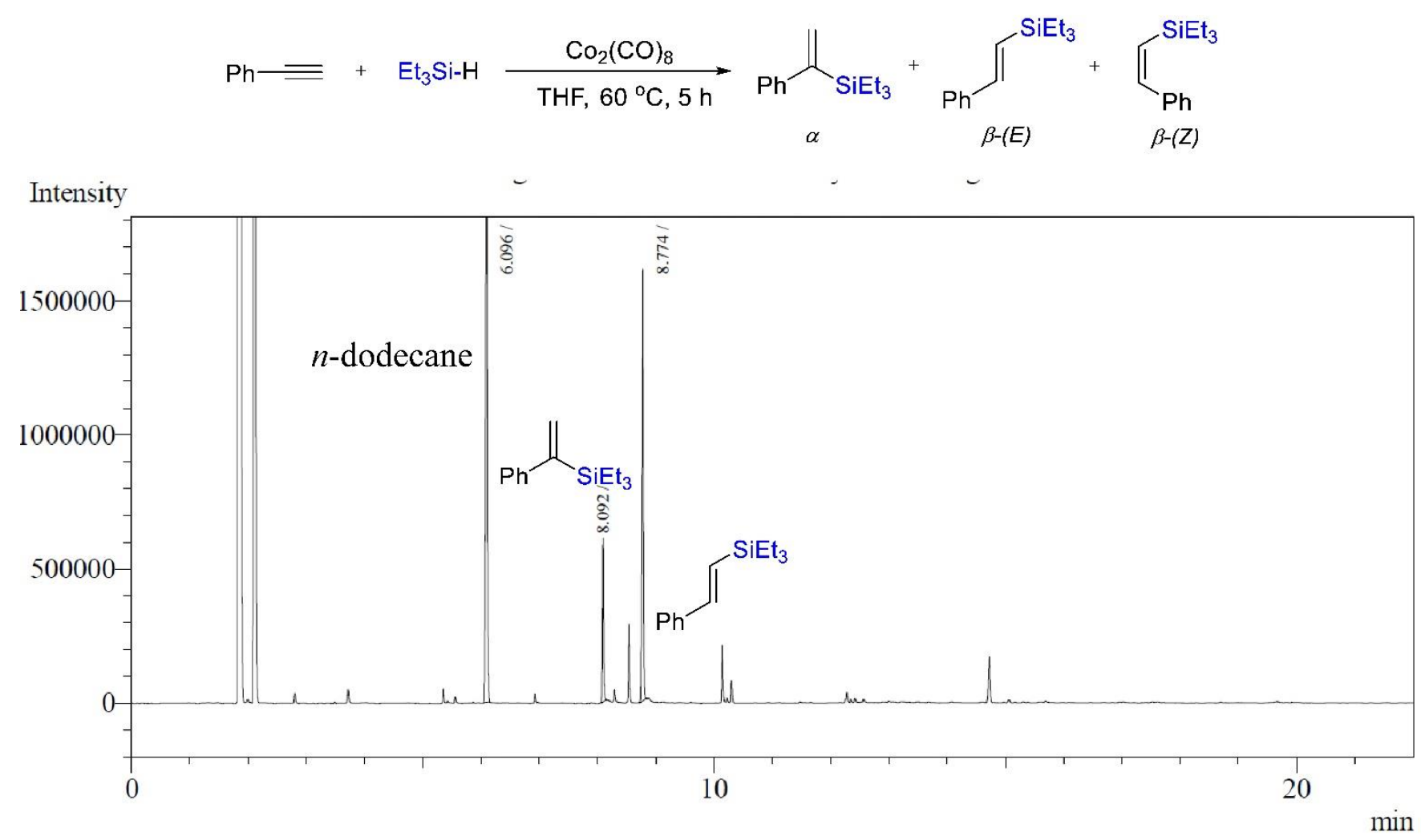

Figure S163. GC graph of the reaction of phenyl acetylene with triethylsilane catalyzed by $\mathrm{Co}_{2}(\mathrm{CO})_{8}$. 

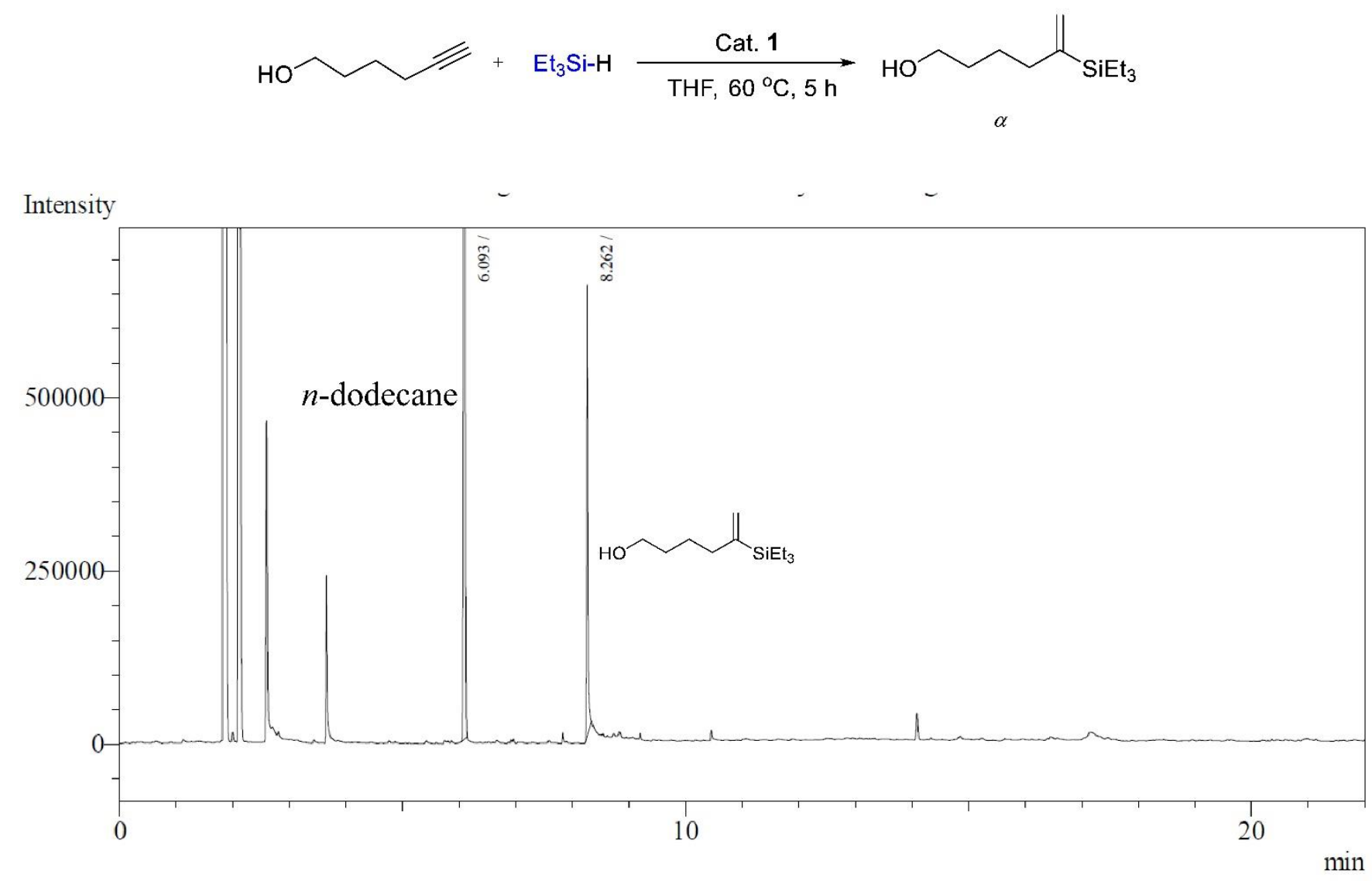

Figure S164. GC graph of the reaction of hex-5-yn-1-ol with triethylsilane catalyzed by $\mathbf{1}$. 

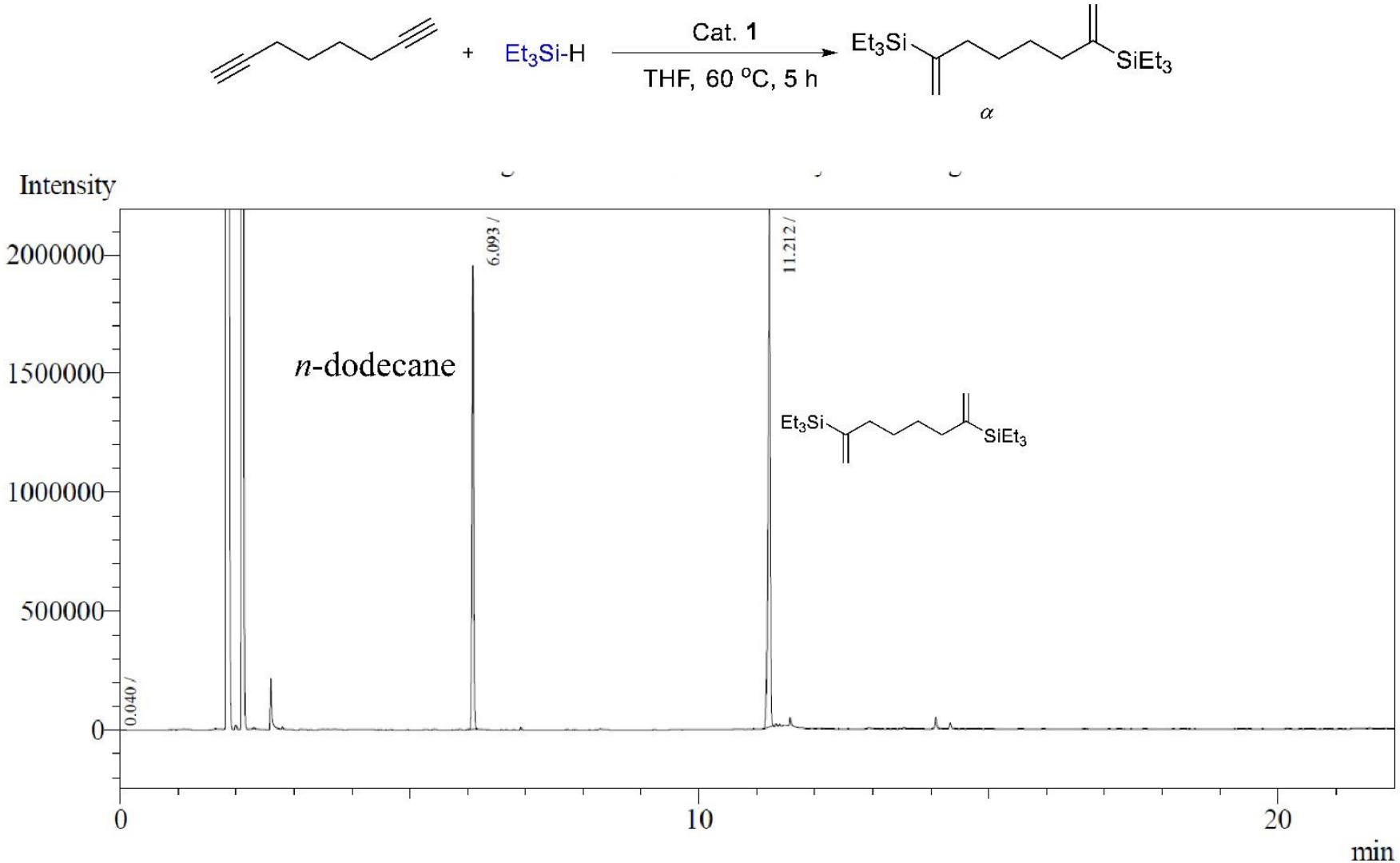

Figure S165. GC graph of the reaction of octa-1,7-diyne with triethylsilane catalyzed by $\mathbf{1}$. 

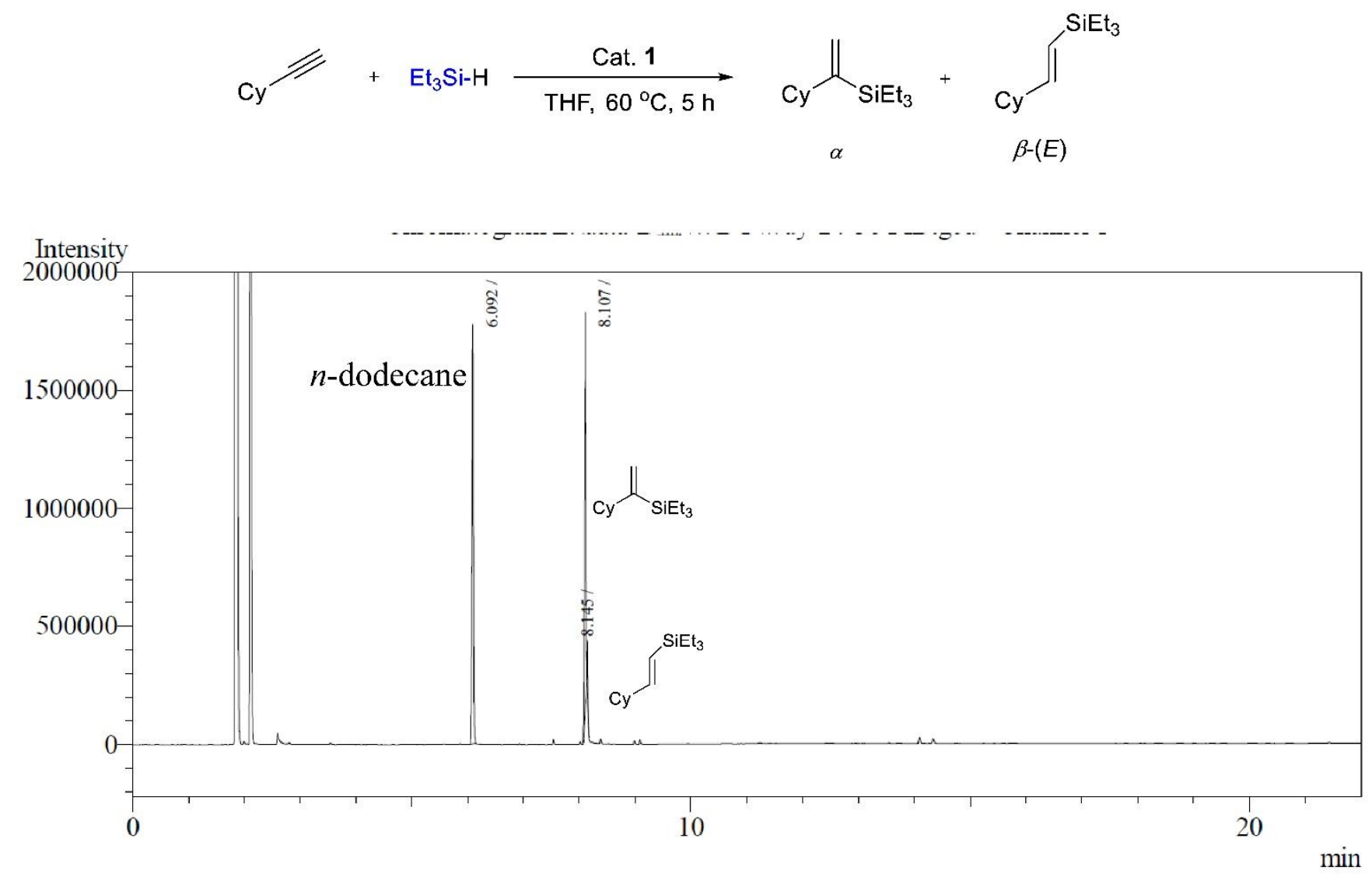

Figure S166. GC graph of the reaction of ethynylcyclohexane with triethylsilane catalyzed by $\mathbf{1}$. 

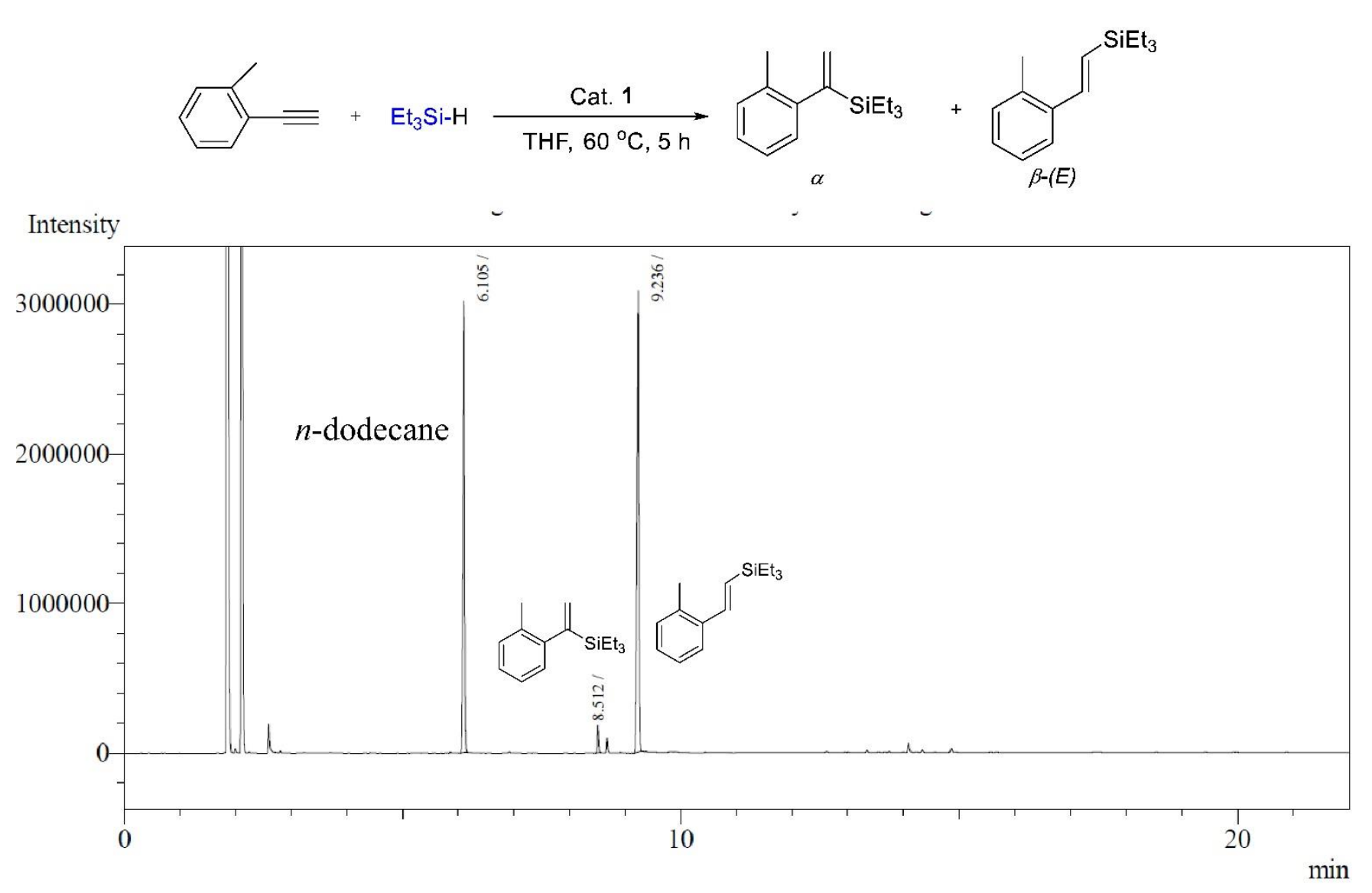

Figure S167. GC graph of the reaction of 1-ethynyl-2-methylbenzene with triethylsilane catalyzed by 1. 

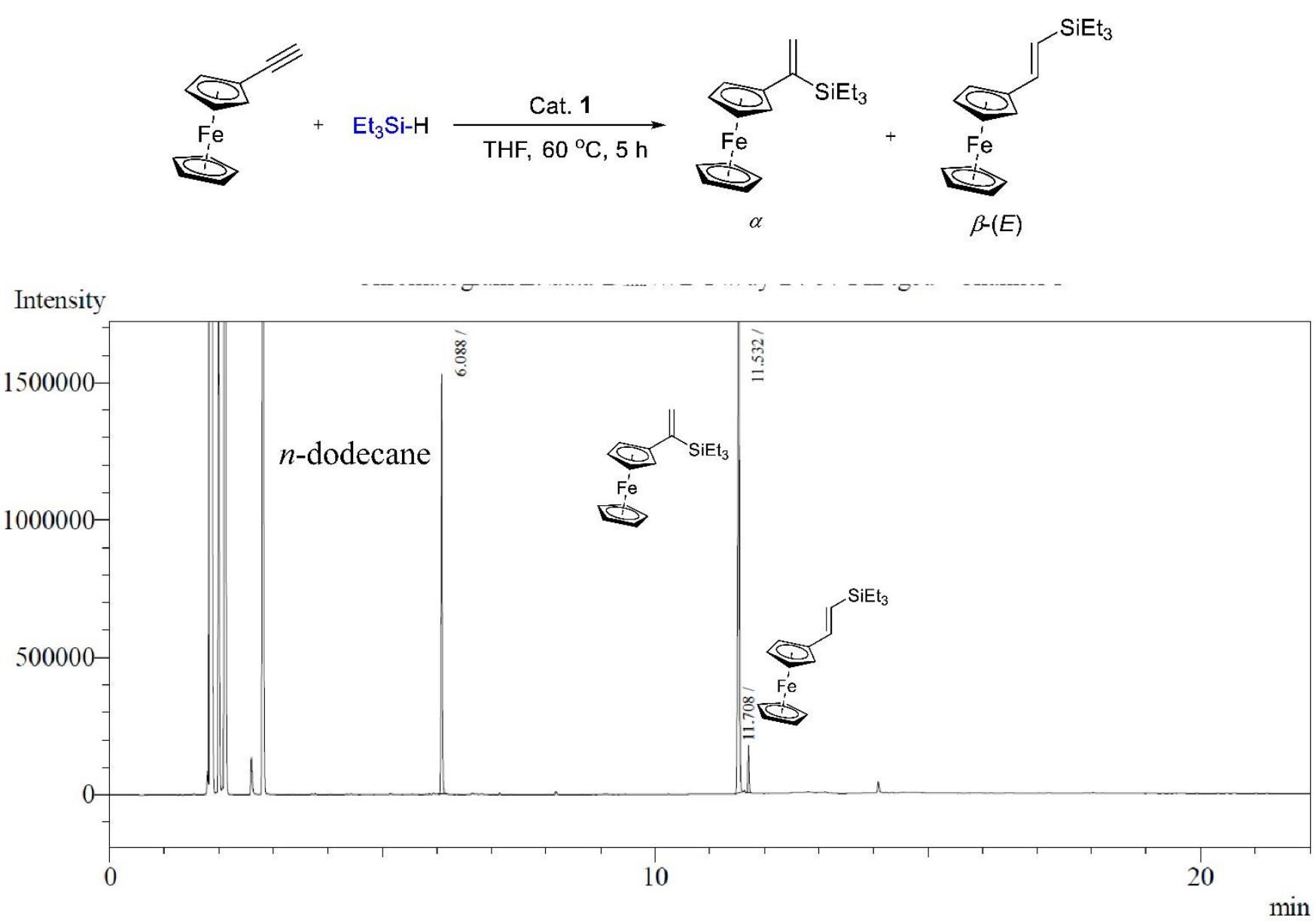

Figure S168. GC graph of the reaction of ethynylferrocene with triethylsilane catalyzed by $\mathbf{1}$. 

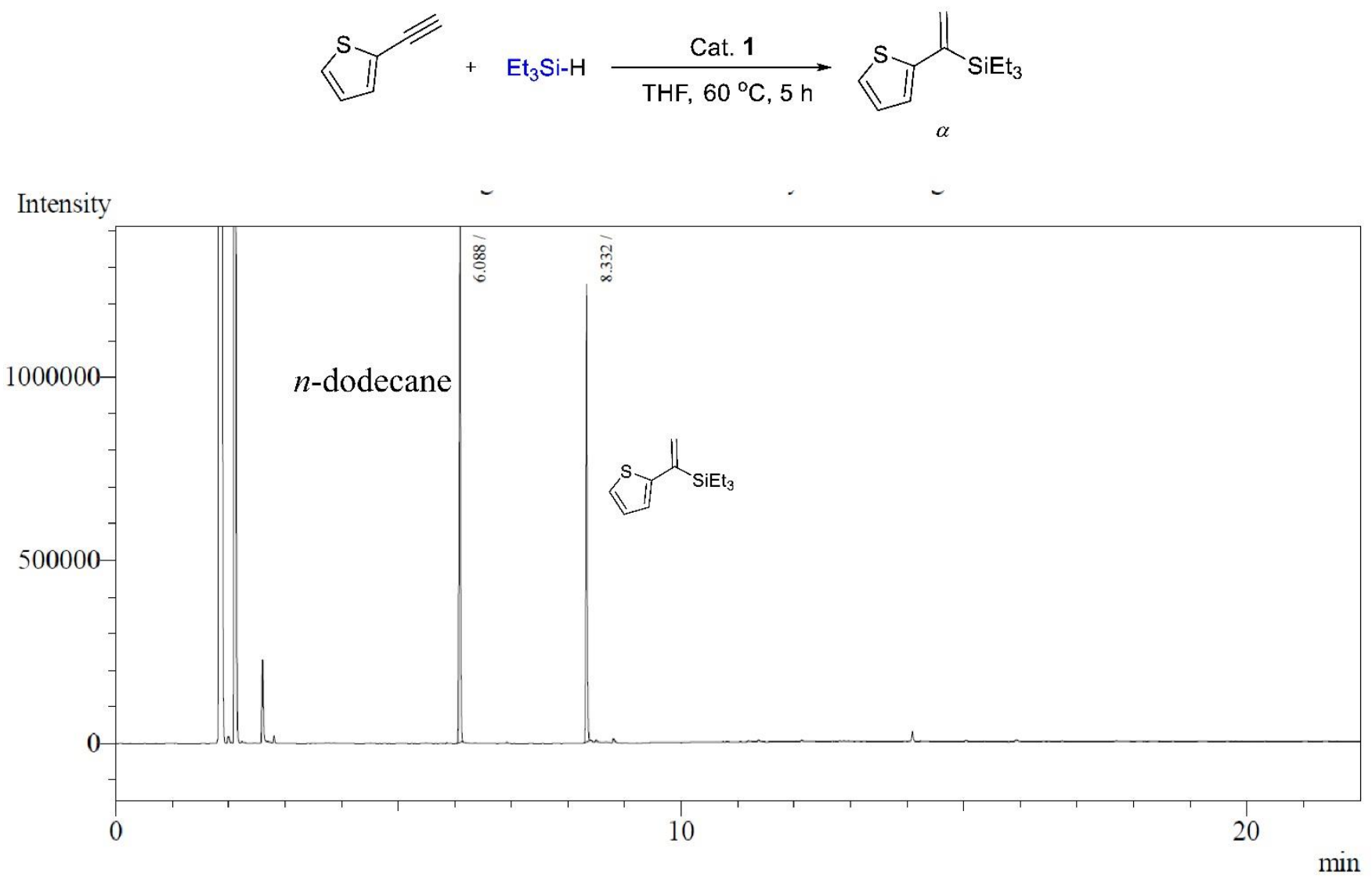

Figure S169. GC graph of the reaction of 2-ethynylthiophene with triethylsilane catalyzed by 1. 

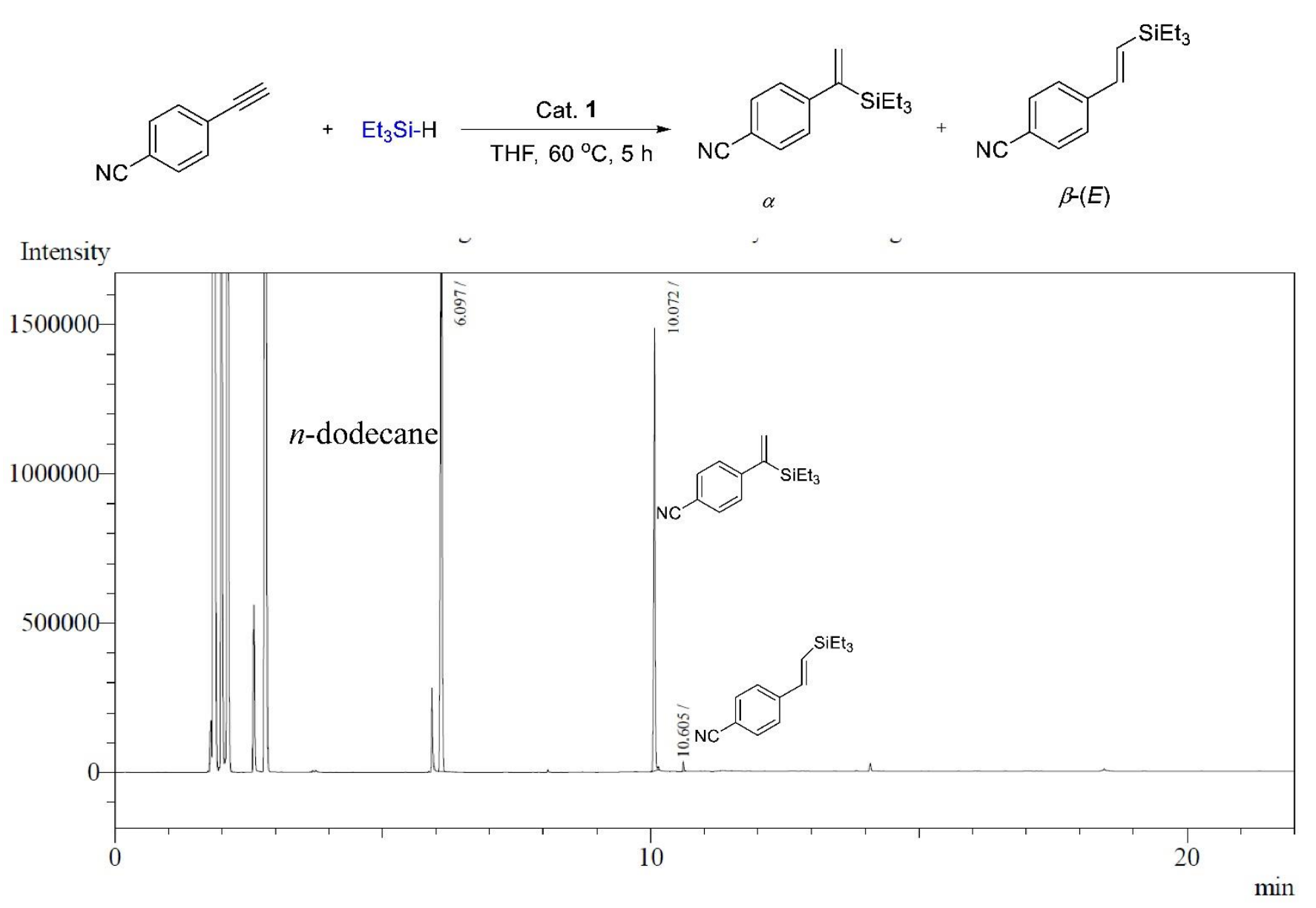

Figure S170. GC graph of the reaction of 4-ethynylbenzonitrile with triethylsilane catalyzed by $\mathbf{1}$. 

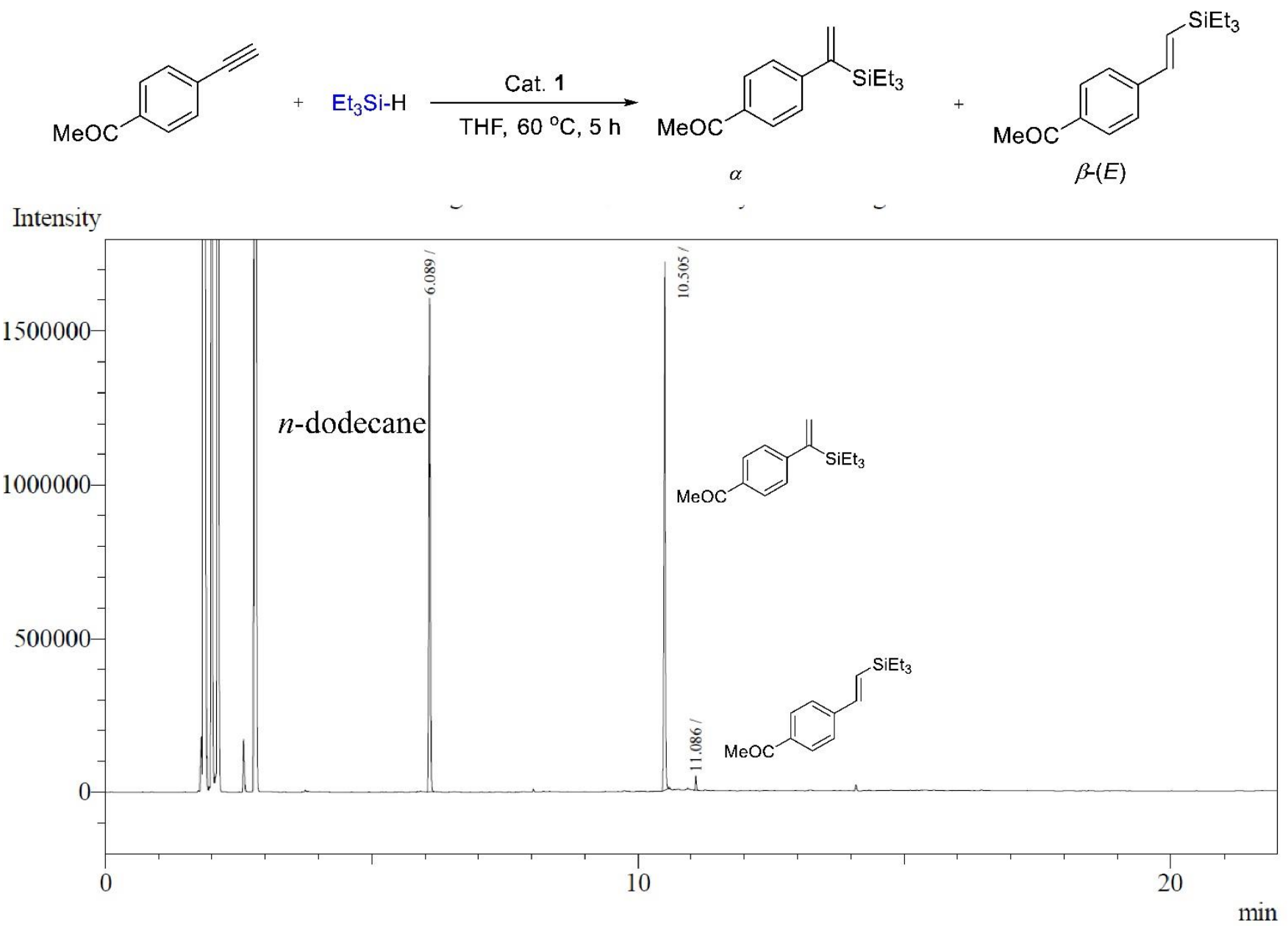

Figure S171. GC graph of the reaction of 1-(4-ethynylphenyl)ethan-1-one with triethylsilane catalyzed by $\mathbf{1}$. 

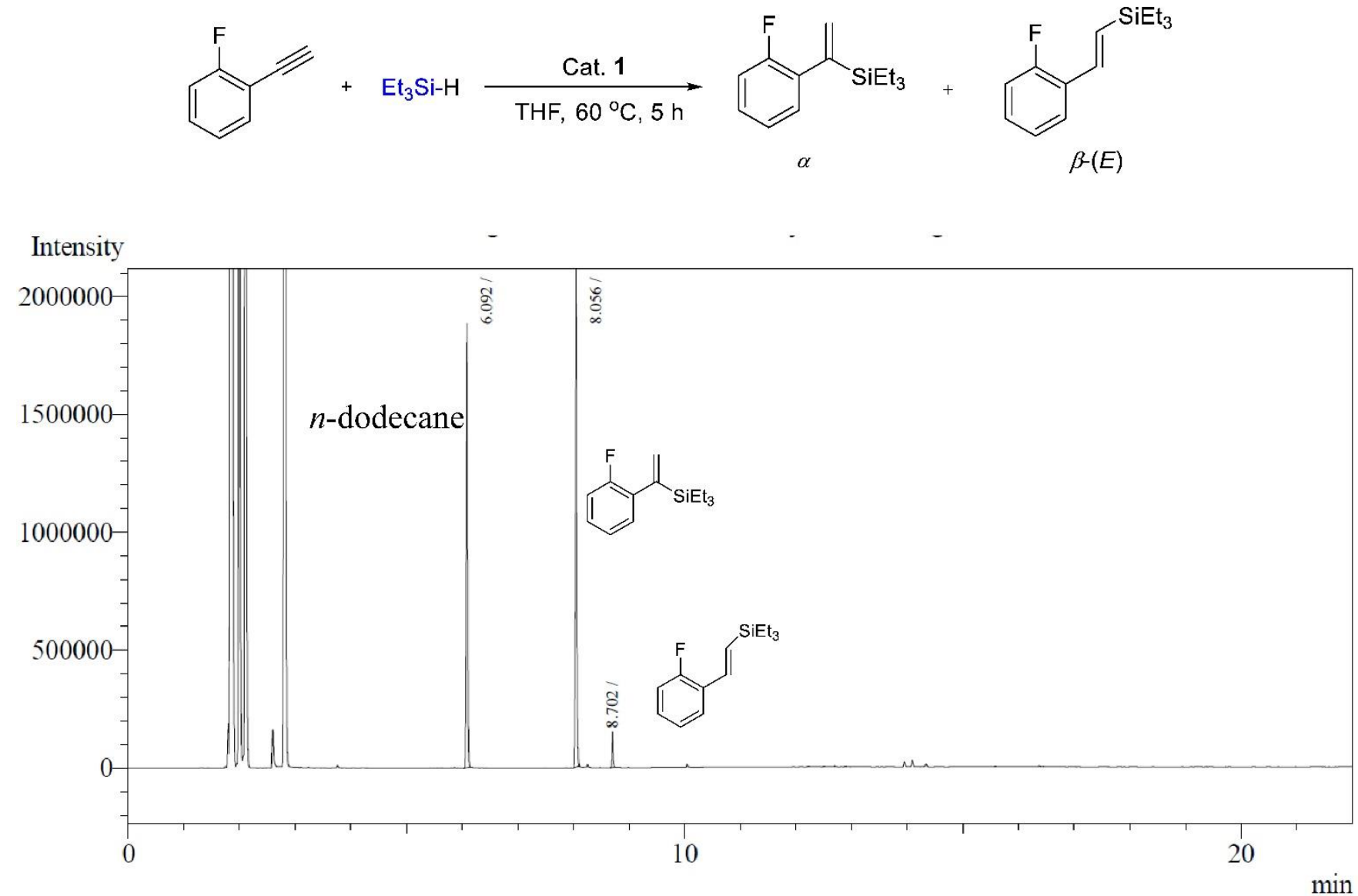

Figure S172. GC graph of the reaction of 1-ethynyl-2-fluorobenzene with triethylsilane catalyzed by $\mathbf{1}$. 

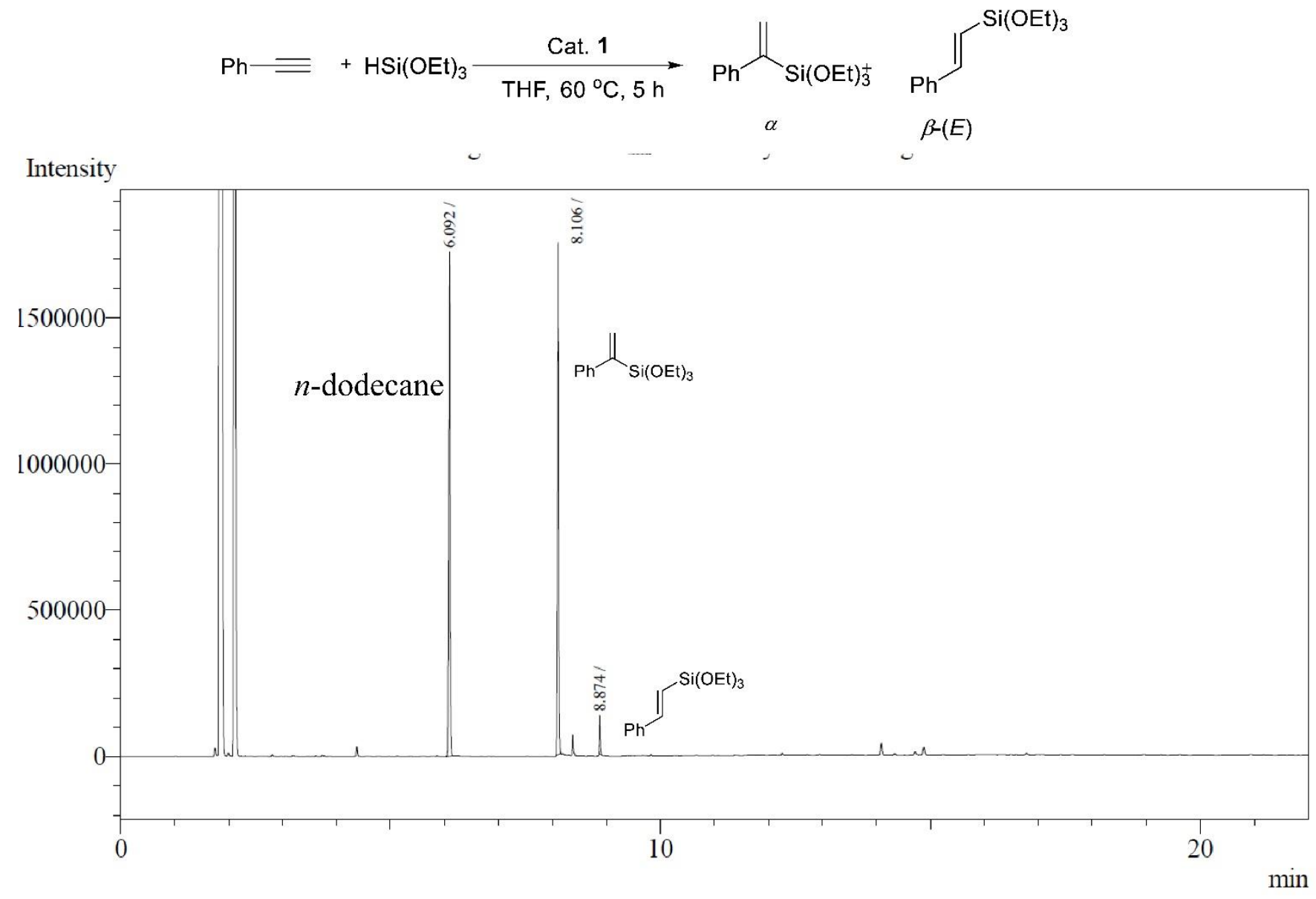

Figure S173. GC graph of the reaction of phenyl acetylene with triethoxysilane catalyzed by $\mathbf{1}$. 


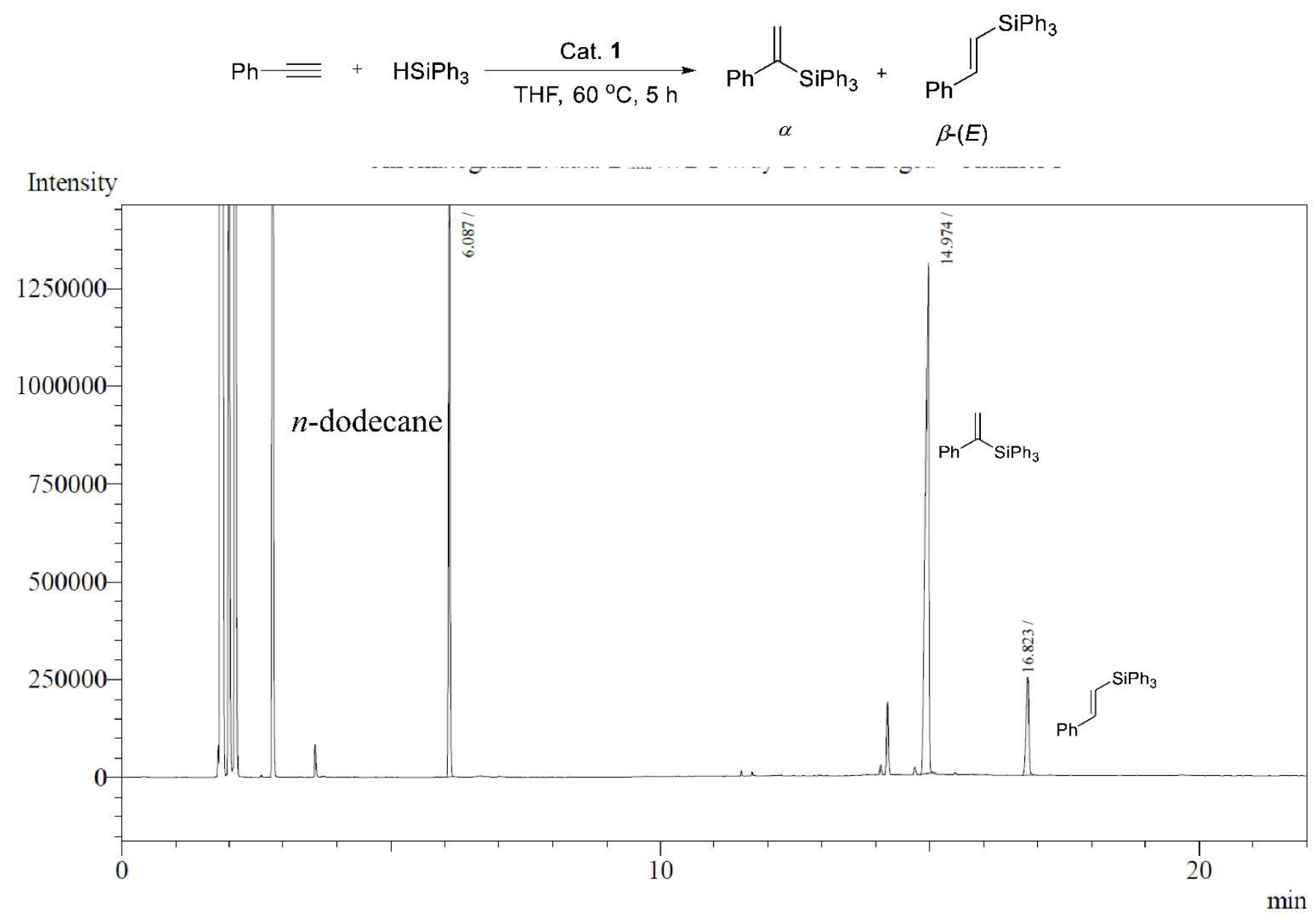

Figure S174. GC graph of the reaction of phenyl acetylene with triphenylsilane catalyzed by $\mathbf{1}$. 


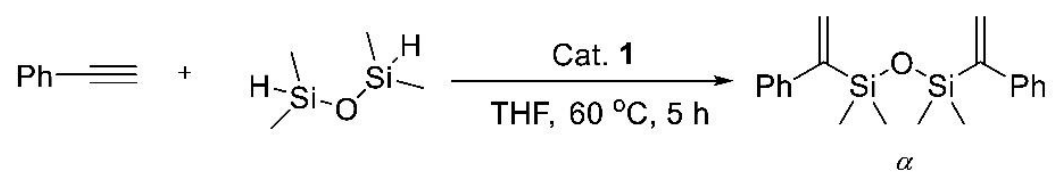

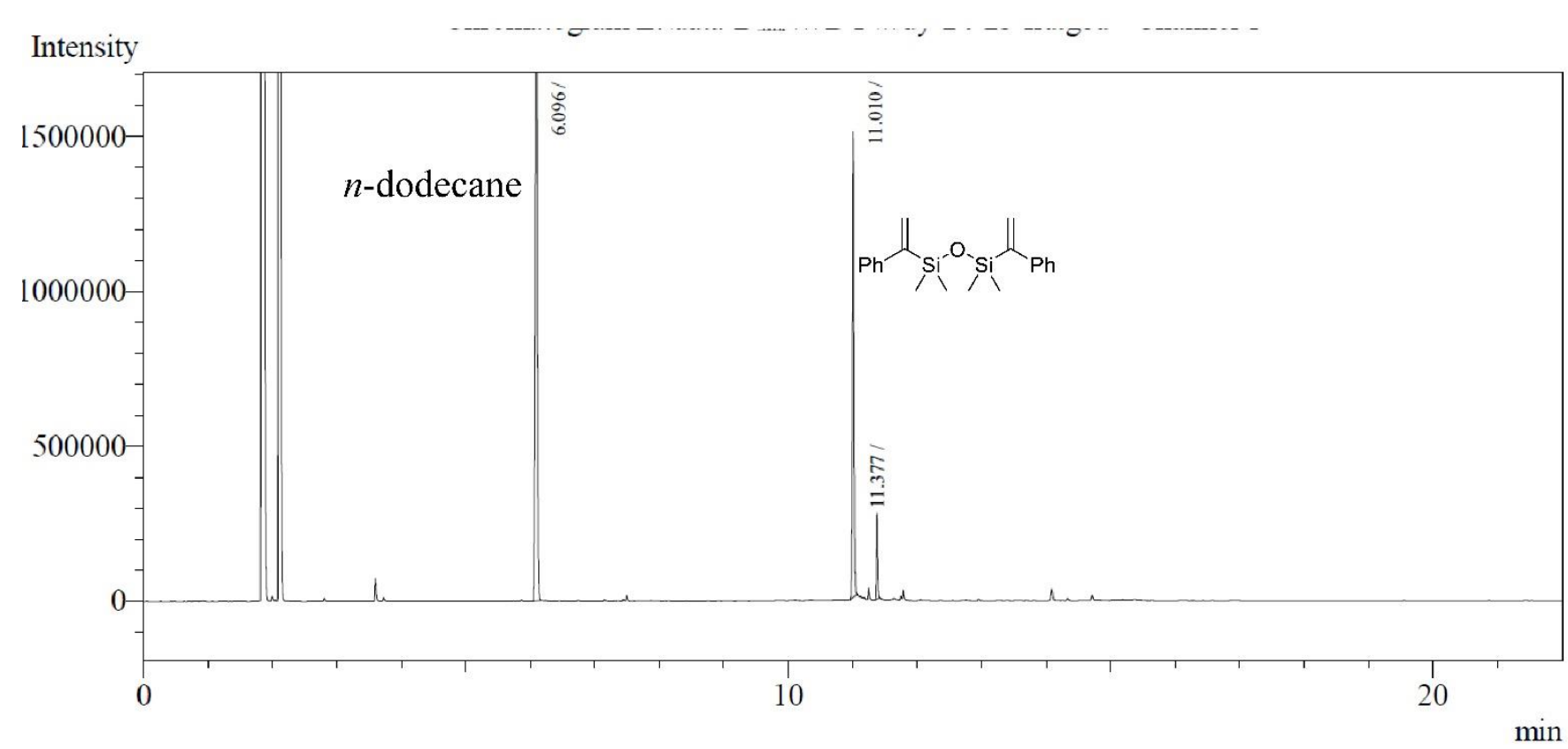

Figure S175. GC graph of the reaction of phenyl acetylene with 1,1,3,3-tetramethyldisiloxane catalyzed by $\mathbf{1}$. 


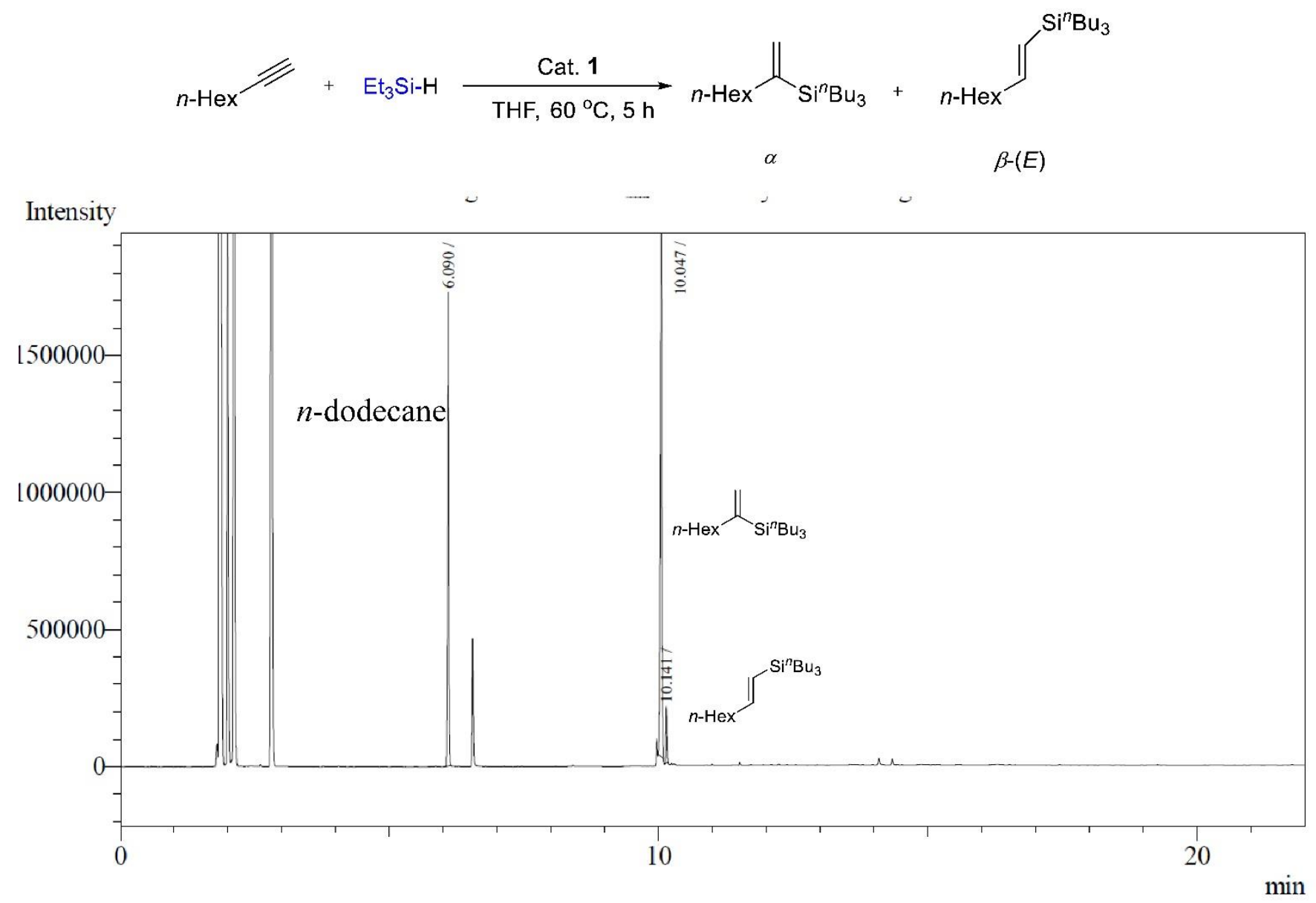

Figure S176. GC graph of the reaction of oct-1-yne with triethylsilane catalyzed by $\mathbf{1}$. 


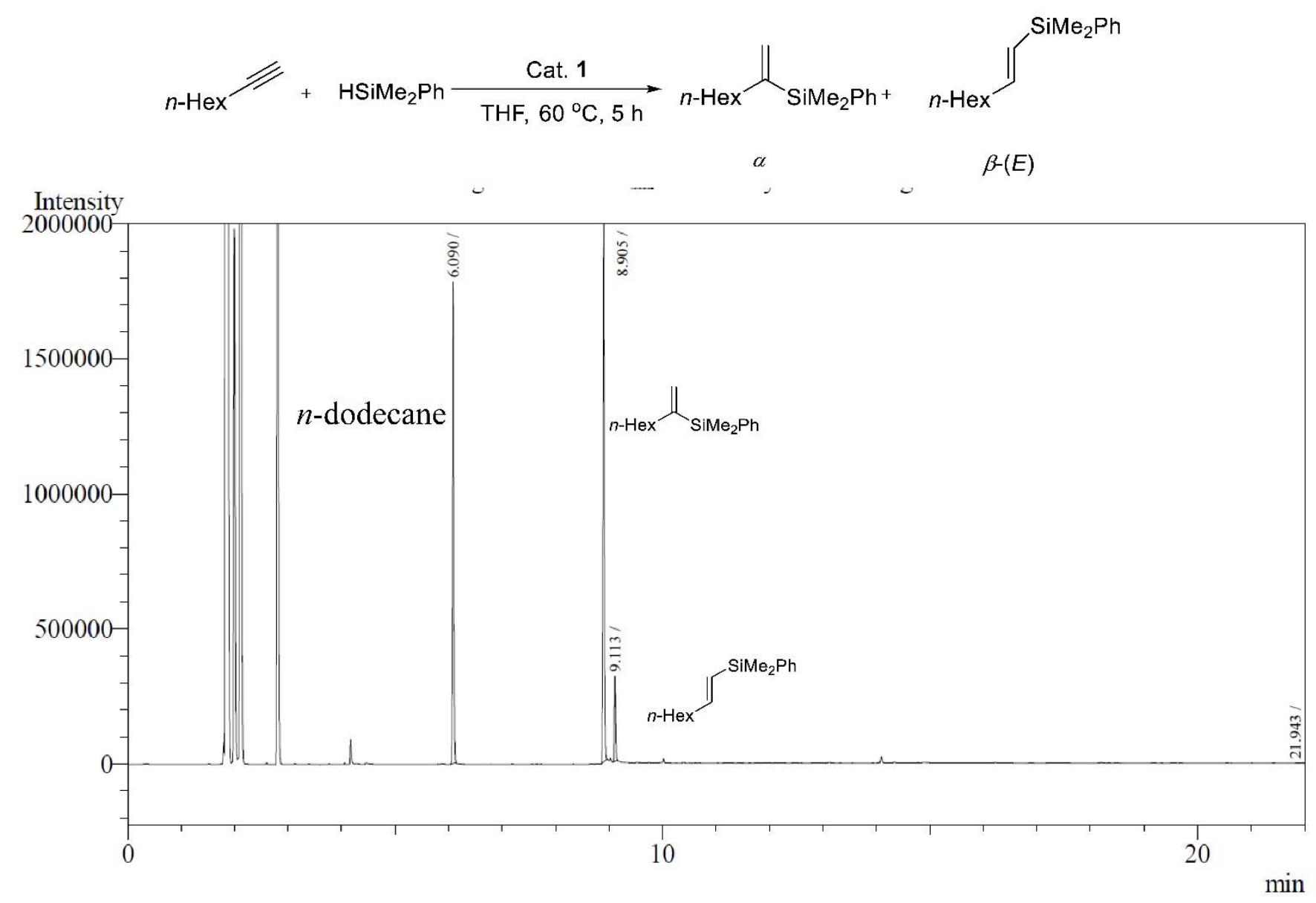

Figure S177. GC graph of the reaction of oct-1-yne with dimethyl(phenyl)silane catalyzed by $\mathbf{1}$. 


$$
n \text {-Hex }{ }_{+} \mathrm{HSiPh}_{3} \underset{\alpha}{\frac{\mathrm{Cat.1}}{\mathrm{THF}, 60^{\circ} \mathrm{C}, 5 \mathrm{~h}}}{ }_{n \text {-Hex }} \|_{\mathrm{SiPh}_{3}}+\underset{n-\mathrm{Hex}}{\mathrm{SiPh}_{3}}
$$

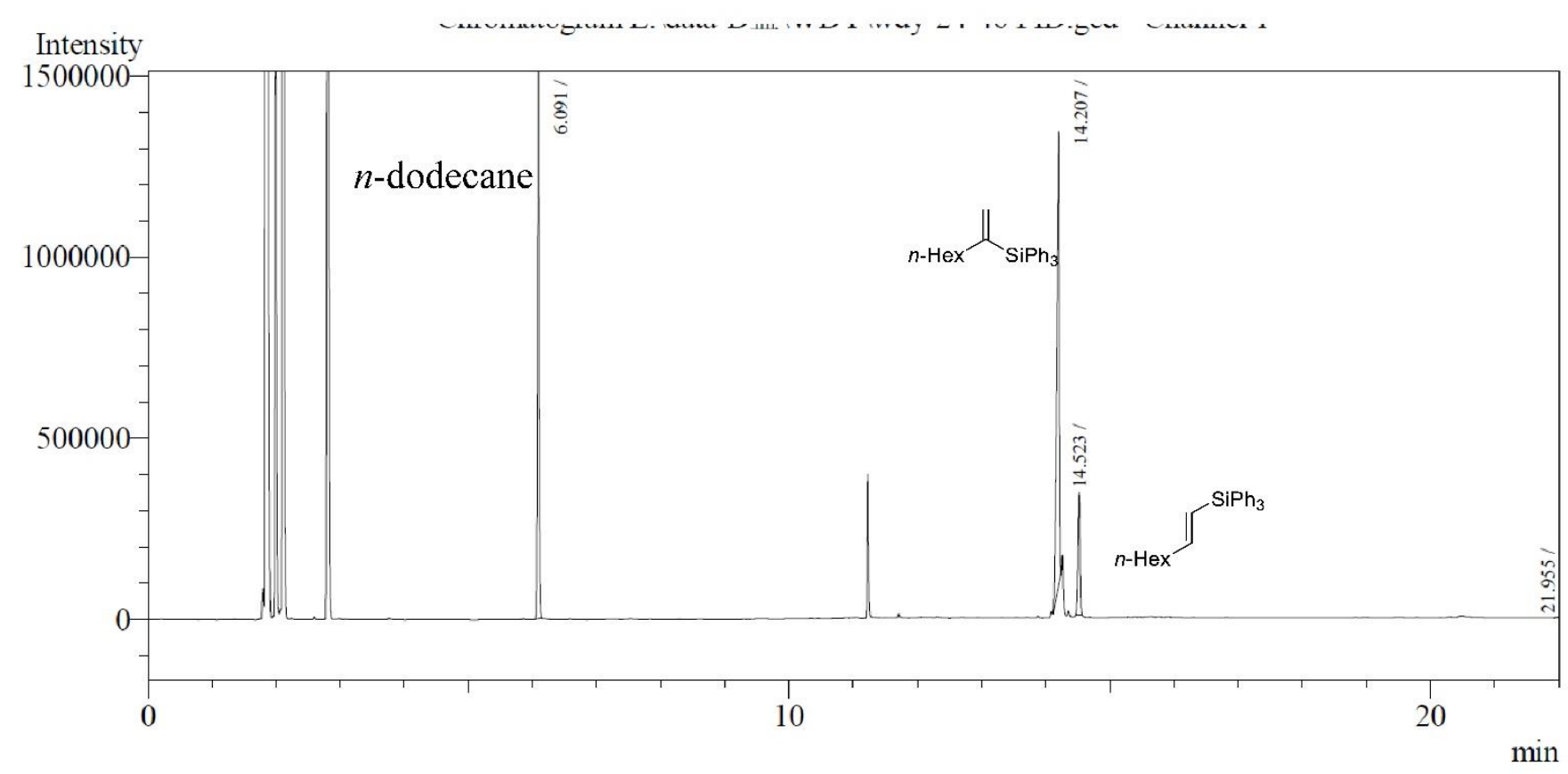

Figure S178. GC graph of the reaction of oct-1-yne with triphenylsilane catalyzed by $\mathbf{1}$. 

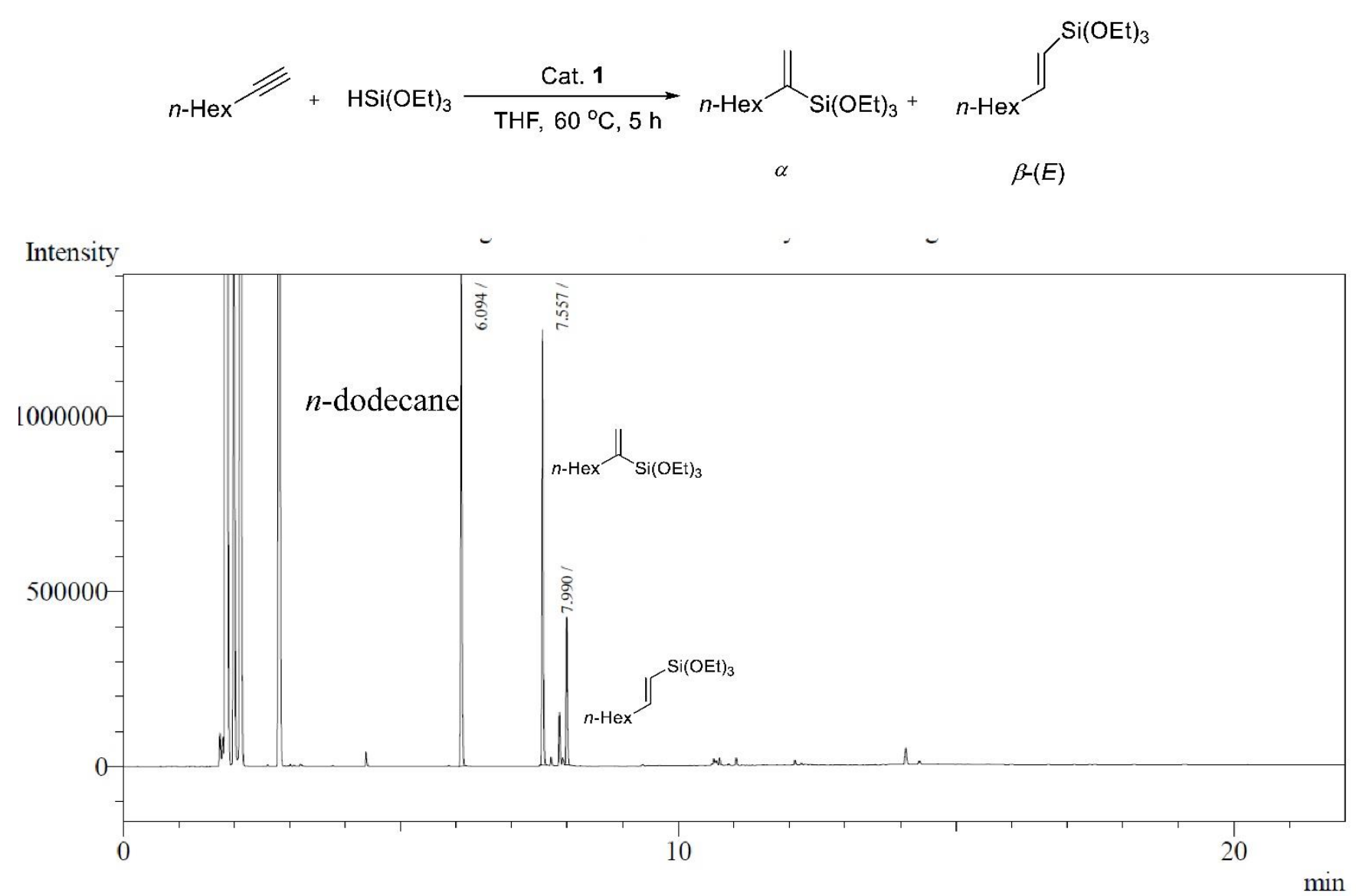

Figure S179. GC graph of the reaction of oct-1-yne with triethoxysilane catalyzed by $\mathbf{1}$. 


$$
\mathrm{Ph} \equiv+\mathrm{H}_{3} \mathrm{SiPh} \frac{\text { Cat. } 1}{\mathrm{THF}, 60^{\circ} \mathrm{C}, 5 \mathrm{~h}} \underset{\alpha}{\mathrm{Ph}} \underset{\beta-(E)}{\mathrm{SiH}_{2} \mathrm{Ph}}+\mathrm{Ph} \underset{\mathrm{SiH}_{2} \mathrm{Ph}}{\longrightarrow}
$$

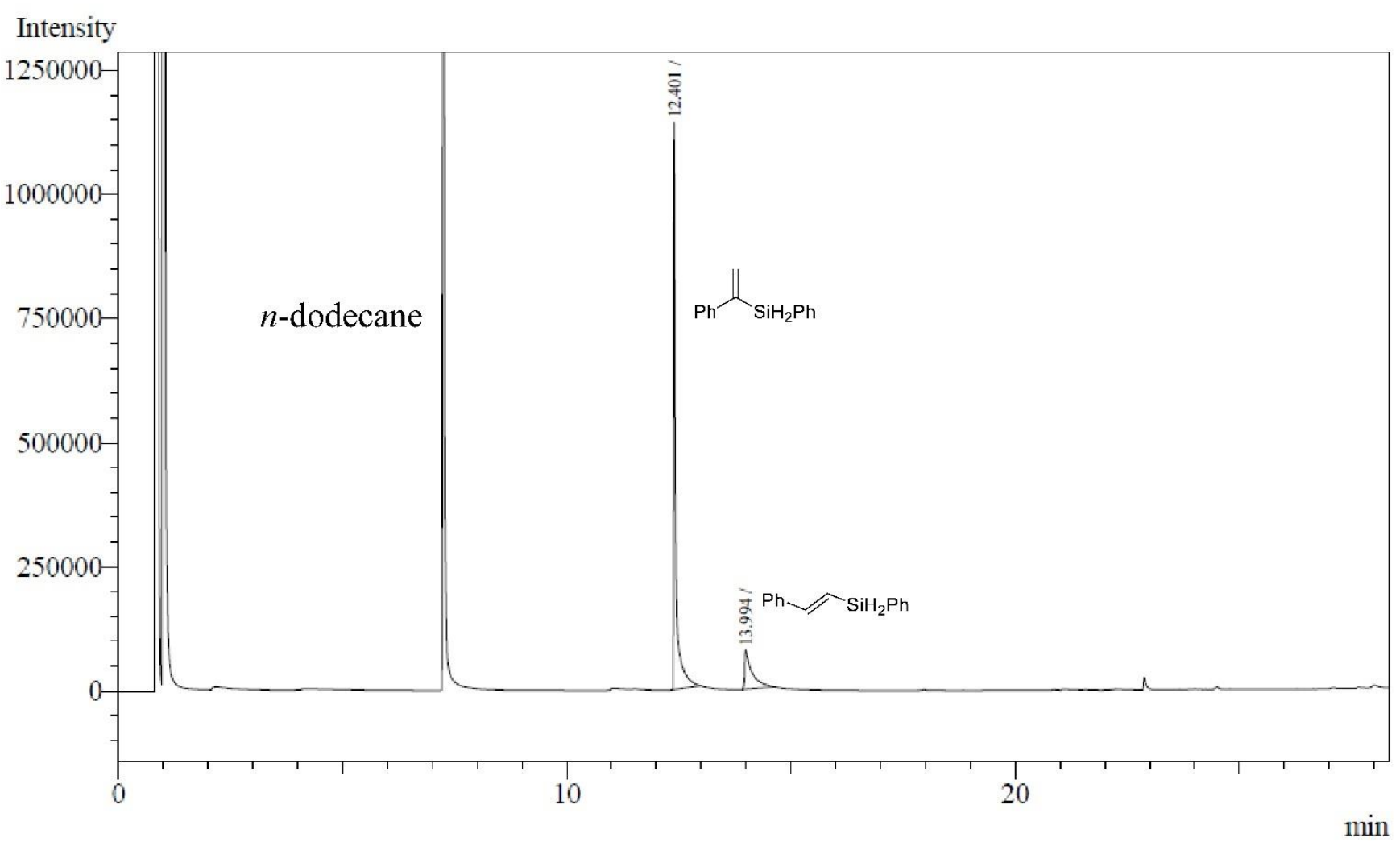

Figure S180. GC graph of the reaction of phenyl acetylene with phenylsilane catalyzed by $\mathbf{1}$. 


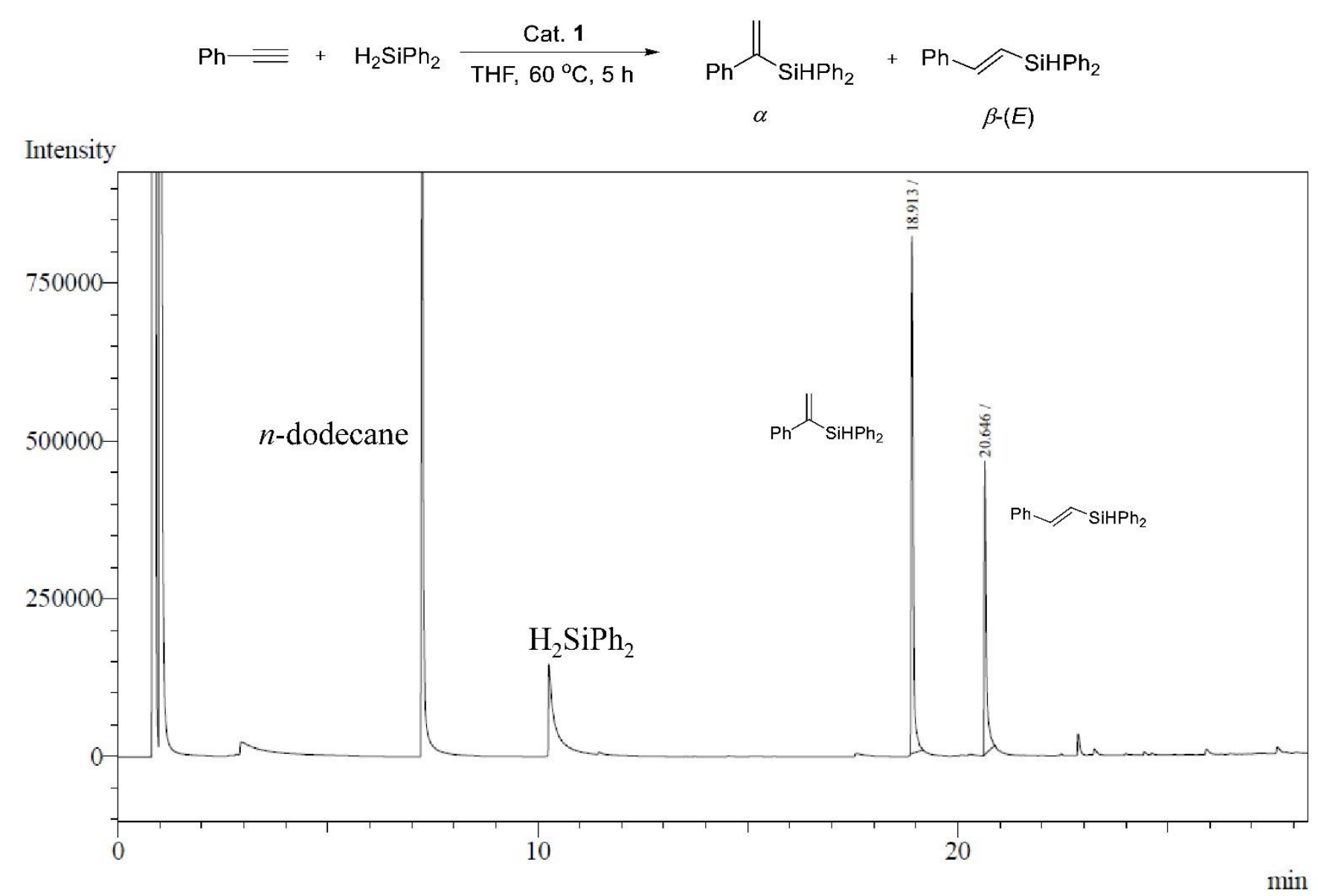

Figure S181. GC graph of the reaction of phenyl acetylene with diphenylsilane catalyzed by $\mathbf{1}$. 